UNIVERSIDADE DE SÃO PAULO

FACULDADE DE FILOSOFIA, LETRAS E CIÊNCIAS HUMANAS

DEPARTAMENTO DE LINGUÍSTICA

PROGRAMA DE PÓS-GRADUAÇÃO EM SEMIÓTICA E LINGUÍSTICA GERAL

LUCIANA SANCHEZ-MENDES

\title{
A Modificação de Grau em Karitiana
}

[ EXEMPLAR REVISADO ]

São Paulo

2014 


\section{A Modificação de Grau em Karitiana}

Tese apresentada ao Programa de Pós-Graduação em Semiótica e Linguística Geral do Departamento de Linguística da Faculdade de Filosofia, Letras e Ciências Humanas da Universidade de São Paulo para obtenção do título de doutora em Linguística com co-orientação de tese com a Université Paris 8.

Orientadora: Profa. Dra. Ana Müller

Co-orientadora: Profa. Dra. Brenda Laca

EXEMPLAR REVISADO

"De acordo" do orientador:

Orientadora: Profa. Dra. Ana Muller

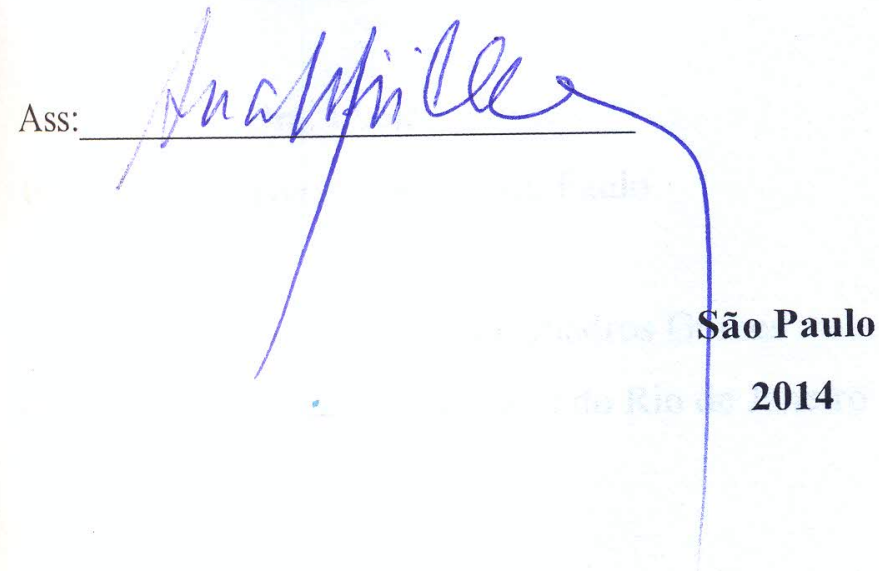




\section{LUCIANA SANCHEZ-MENDES}

\section{A Modificação de Grau em Karitiana}

Tese apresentada ao Programa de Pós-Graduação em Semiótica e Linguística Geral do Departamento de Linguística da Faculdade de Filosofia, Letras e Ciências Humanas da Universidade de São Paulo para obtenção de título de doutora em Linguística com co-orientação de tese com a Université Paris 8.

Orientadora: Profa. Dra. Ana Müller

Co-orientadora: Prfa. Dra. Brenda Laca

\section{[ EXEMPLAR REVISADO - Versão Original Disponível na Biblioteca da Unidade]}

Aprovada em 22 de abril de 2014

\section{Banca Examinadora}

Presidente: Profa. Dra. Ana Müller

Instituição: Universidade de São Paulo

Titular: Profa. Dra. Brenda Laca

Instituição: Université Paris 8

Titular: Profa. Dra. Elena Soare

Instituição: Université Paris 8

Titular: Profa. Dra. Luciana Storto

Instituição: Universidade de São Paulo

Titular: Profa. Dra. Ana Paula Quadros Gomes

Instituição: Universidade Federal do Rio de Janeiro 
ESTA TESE FOI FINANCIADA PELA FAPESP - FUNDAÇÃO DE AMPARO À PESQUISA DO ESTADO DE SÃO PAULO

PROCESSO 09/17185-8 
"I reject the contention that an important theoretical difference exists between formal and natural languages."

"Eu rejeito a afirmação de que exista uma diferença teórica importante entre as línguas naturais e formais."

Montague (1970, tradução nossa) 


\section{AGRADECIMENTOS}

O linguista Greg Carlson, em seu famoso livro Reference to kinds in English (Carlson 1980), diz que não se deve levar muito a sério o fato de haver apenas o seu nome na capa do livro. $\mathrm{O}$ autor diz isso para iniciar seus agradecimentos. No mesmo espírito, quero dedicar esta parte para agradecer às pessoas que contribuíram de alguma forma para que este trabalho fosse realizado, as quais podem se considerar um pouco autoras desta tese. Todos os erros, claro, são de minha responsabilidade.

Quero agradecer primeiramente à professora Ana Müller, minha orientadora de muitos anos, que eu tanto admiro. A Ana foi minha orientadora em muitos aspectos. Sempre me guiou para me tornar uma linguista, uma professora, uma pesquisadora melhor. Sua disponibilidade em me aconselhar em tantas coisas fizeram da Ana a minha mentora acadêmica. Além disso, com o passar dos anos, tive o prazer de ser coautora da Ana e passar tardes deliciosas discutindo enigmas linguísticos, momentos de muito aprendizado para mim. Agradeço à Ana por ter se tornado, além de tudo, minha amiga e minha colega de trabalho, o que é uma grande honra.

Agradeço à minha co-orientadora, a professora Brenda Laca, por aceitar orientar este trabalho. A Brenda fez a minha estadia em Paris bastante produtiva, sempre me apoiando a fazer cursos interessantes e tentar congressos mais difíceis e instigantes. Além disso, mesmo de longe, a Brenda sempre acompanhou e orientou o meu percurso acadêmico.

Quero agradecer também à grande colaboradora deste trabalho, a professora Luciana Storto. A Luciana contribuiu com este trabalho desde o início, com sua solidariedade em nos ajudar com a língua Karitiana, seja nos apresentado para a comunidade, seja revisando nossos dados. A Luciana fez parte da minha banca de qualificação e fez contribuições muito importantes que hoje fazem parte deste texto. Agradeço ainda à Ana Gomes, pelo incentivo, pela ajuda, pela arguição na banca de qualificação. Eu tive a sorte de contar com essas mulheres incríveis no meu caminho acadêmico.

Quero agradecer a todos os professores do Departamento de Linguística da USP, com quem tive aulas e que fizeram parte diretamente da linguista que eu me tornei. Agradecimentos especiais a Esmeralda Negrão, Cristina Altman, Marcelo Ferreira, Marcello Modesto, Ana Scher. Ainda no grupo do staff da USP, quero agradecer ao Ben Hur, ao Robson e à Érica pelo apoio institucional. Sem a Érica, minha vida como pós-graduanda seria muito mais difícil, e muito mais chata também.

Quero ainda fazer um agradecimento à instituição Universidade de São Paulo como um todo. Ter estudado na USP mudou para sempre a minha vida e é um grande orgulho para mim. A USP fez parte do que eu fui e do que me tornei. Entrei na USP com 18 anos, morei no Crusp, trabalhei em um museu da USP, sempre almocei e jantei no bandeijão da USP (cefé da manhã também, depois que ficou disponível), fui ao cinema no Cinusp, ao teatro no Tusp, assisti ao Coralusp. Foi no contexto da universidade que eu me tornei linguista, professora, mulher, cidadã.

Quero agradecer às professoras Carmen Dobrovie-Sorin e Lucia Tovena pela excelente recepção em Paris. Agradecimentos especiais à Marta Donazzan, que me ajudou em muitos momentos a me enturmar no novo ambiente. Agradeço a Heather Burnett e Daniel Lassiter pelo curso "Gradability, Scale Structure, and Vagueness", no 25th European Summer School in Logic, Language and Information (ESSLLI), realizado em Düsseldorf, na Alemanha. Suas 
aulas foram esclarecedoras para mim.

Quero agradecer aos amigos da pós-graduação que me acompanharam em cursos, discussões, cafés e viagens. Lídia, Nize, Letícia, Julio, Roberlei, Marcus, Ivan, Indaiá, Fátima, Renato, Aline, Paula, Vitor, Rafael, Wânia. Agradecimentos especiais à Lara, minha companheira de escalas, à Fernanda, minha companheira de viagens, e à Karin, minha companheira de trabalho de campo. Agradeço também o apoio dos amigos da Iniciação Científica Ju, Thiago, Lucas e Fernando.

Agradeço aos meus amigos não linguistas que também me apoiaram nesse percurso. À Cris, que continuou se mantendo firme como meu anjo da guarda, para quem eu posso pedir qualquer coisa em qualquer horário. À Cecília e ao Mário, meus amigos do coração. Ficar longe deles é muito dificil. À Ana Umeda, minha companheira de farras e discussões artísticas. Ao Rodrigo, meu amigo de infância, que já me conhece tão bem e torce tanto por mim. Ao Flávio, por ser meu amigo de viagens fantásticas. Ao Renato, que trouxe o lápis que faltava para a minha coleção. Aos patagônicos Flá, Alex, Natan, Rô, Mari e Dê, companheiros de aventuras. Ao André pela amizade de anos e de sempre. À Thalita e ao André, meus companheiros em Barcelona. Às minhas roomates Jéssica, Fernanda e Cris (de novo!) pelo companheirismo e amizade. Aos meus ex-roomates Roger, Maurício e Paula, Carol e Caneta, e Cris (de novo!) pela experiência inesquecível do Clube dos Cinco. Aos amigos de Paris, Carlos e Renan, linguistas paulistas na capital francesa, Flávia e Lizânia, pelos momentos inesquecíveis. Aos meus ex-alunos da FIA e do CPV, com quem aprendi a dar os primeiros passos como professora.

Aos consultores Karitiana com quem trabalhei. João, Milena, Inácio, Sarita, Caros Alberto, Cledson, Maria de Fátima, Luiz, Sandra, Paulo, Edimilson. Agradecimentos especiais ao Elivar e à Marilena, que se tornarm meus amigos. O interesse e a ajuda de vocês me fizeram ficar cada mais envolvida com o estudo da língua Karitiana. Yryhodn!

Agradeço ao Conselho Nacional de Desenvolvimento Científico e Tecnológico (CNPq) pelo apoio financeiro inicial desta pesquisa (processo 141147/2010-5) e à Fundação de Amparo à Pesquisa do Estado de São Paulo (Fapesp) pelo apoio financeiro ao longo do doutorado (processo 09/17185-8). Agradeço ainda à Coordenação de Aperfeiçoamento de Pessoal de Nível Superior (CAPES) pela bolsa que me permitiu estudar em Paris por um ano (processo BEX 3574/11-3). A bolsa fazia parte do projeto CAPES-COFECUB 647/09 "Os Nominais Nus no Português Brasileiro: A interface sintaxe e semântica" coordenado pelas professoras Carmen Dobrovie-Sorin, da Université Paris 7 e Roberta Pires de Oliveira, da Universidade Federal de Santa Catarina. Agradeço à Roberta pela disponibilidade em me ajudar na documentação da bolsa.

Por fim, agradeço a minha família, meus pais João e Lúcia, pelo apoio incondicional em todos os momentos. Pela compreensão, pelo carinho, pela ajuda. A minha irmã Jordana, pela ajuda estratégica em Franca mandando documentos ou qualquer coisa que eu precisasse de lá. Ter de viver em cidades diferentes durante o doutorado não teria sido possível sem a ajuda dessas três pessoas.

Quero deixar ainda um agradecimento ao meu avô Antônio (in memorian) de quem herdei o Sanchez com $Z$. Meu avô nunca soube direito o que eu fazia, mas sempre me olhou com a ternura de quem achava, de alguma forma, que o que eu fazia era importante. 


\section{RESUMO}

SANCHEZ-MENDES, L. A Modificação de Grau em Karitiana. 259f. - Tese (Doutorado em Linguística) - Universidade de São Paulo e Université Paris 8, 2014.

O objetivo desta tese é apresentar uma análise para a modificação de grau em Karitiana. Karitiana é uma língua da tronco Tupi, família Arikém, falada por aproximadamente 320 pessoas em uma área demarcada no noroeste do Brasil (Storto e Vander Velden 2005). A tese está dividida em quatro capítulos.

O primeiro capítulo é sobre o contexto teórico e sobre algumas características da língua. O conexto teórico é relativo à Semântica Formal. Apresentamos uma introdução sobre esse paradigma teórico e sobre as ferramentas utilizadas para a composição semântica (Heim e Kratzer 1998). A parte sobre o Karitiana apresenta algumas características da língua, como o modo, o tempo, o aspecto e os sintagmas nominais (Storto 1999 e Müller et al. 2006).

O segundo capítulo é sobre a metodologia utilizada para a realização dos trabalhos de campo. Discutimos a importância da elicitação controlada de dados para uma pesquisa em Linguística Formal (Matthewson 2004). Discutimos também algumas questões práticas a respeito dos trabalho de campo, como a negociação com os membros da comunidade que fala a língua, assim como os princípios éticos aplicados à pesquisa linguística (Rice 2012).

O terceiro capítulo é a respeito dos sintagmas verbais em Karitiana. Primeiramente, fazemos uma discussão sobre a teoria do aspecto lexical (Aktionsart) (Vendler 1957, Verkuyl 1996, Krifka 1998 e Rothstein 2004). Em seguida, apresentamos uma análise sobre a telicidade, a cumulatividade, e a atomicidade dos predicados verbais em Karitiana. Aplicamos os testes clássicos da literatura linguística sobre a telicidade, como o paradoxo do imperfectivo e as expressões com por/em uma hora. Fazemos também uma análise sobre a cumulatividade e a atomicidade em Karitiana. Os resultados obtidos são os seguintes: (i) alguns testes devem ser adaptados para serem aplicados em Karitiana (considerando-se as propriedades de tempo, aspecto e dos sintagmas nominais na língua); (ii) há uma diferença entre predicados télicos e atélicos em Karitiana; (iii) os predicados verbais eventivos são cumulativos e contáveis na língua.

O quarto capítulo é sobre a modificação de grau em Karitiana realizada pelo advérbio pita(t) 'verdadeiro/muito/completamente/mesmo'. Pita(t) pode aparecer nos domínios 
nominal, adjetival e verbal. Sua interpretação depende do tipo de escala associada ao predicado modificado. Quando pita(t) modifica sintagmas nominais, ele pode ser traduzido por 'verdadeiro'. Quando pita(t) modifica os sintagmas adjetivais, ele é interpretado como 'muito', se o adjetivos for de escala aberta, e 'completamente' se for de escala fechada. No domínio verbal, pita(t) tem uma restrição de distribuição, ele pode aparecer somente com sintagmas com predicados atélicos com uma interpretação de grau, e tem uma interpretação equivalente à do muito do português. Por outro lado, quando se usa pita(t) com predicados télicos (accomplishments e achievements), a interpretação é algo como 'mesmo'.

Para analisar essas características atípicas de pita(t), utilizamos o pano de fundo teórico da Semântica Escalar (Kennedy e McNally 2005, Kennedy 2007). Uma vez que os predicados nominais e verbais não possuem uma escala lexical como os adjetivos graduáveis, propomos uma função de grau Deg que é responsável por fornecer um argumento de grau e uma escala à denotação de nomes e verbos. A escala associada aos predicados nominais é uma escala fechada de precisão e a escala associada aos sintagmas verbais é uma variável, preenchida de acordo com o tipo do verbo. Os predicados atélicos são considerados como predicados que são associados a uma escala aberta e indeterminada, enquanto que os predicados télicos são predicados associados a escalas fechadas e determinadas.

$\mathrm{Na}$ análise, propomos que pita(t) é um modificador de grau que manipula o grau associado ao ponto de corte da escala representado pelo grau $\mathrm{d}_{\mathrm{s}}$ em Kennedy (2007), que é o grau normal quando a escala é aberta e o grau máximo quando a escala é fechada. A tese defendida apoia que se considere o ponto de corte de uma escala como um grau disponível para modificação e para que se considere a indeterminação como um traço importante para a tipologia dos predicados graduáveis.

Palavras-chave: modificação; grau; advérbios; eventos; línguas indígenas. 


\section{RÉSUMÉ}

SANCHEZ-MENDES, L. La Modification de Degré en Karitiana. 259f. - Thèse (Doctorat em Linguistique) - Universidade de São Paulo et Université Paris 8, 2014.

Le but de cette thèse est de présenter une analyse sur la modification de degré en Karitiana. Karitiana est une langue de la famille Tupi, branche Arikém, parlée par environ 320 personnes dans une zone délimitée au nord-ouest du Brésil (Storto et Vander Velden 2005). La thèse est diviseé en quatre chapitres. La thèse est diviseé en quatre chapitres.

Le premier chapitre porte sur le contexte théorique et sur quelques caractéristiques de la langue Karitiana. Le contexte théorique présenté est relatif à Semantique Formelle. On présente une introduction sur cette paradigme théorique et sur les outils utilisé pour la composition sémantique (Heim et Kratzer 1998). La partie sur le Karitiana présente quelques catégories grammaticales de cette langue, comme le mode, le temps, l'aspect et les syntagmes nominaux (Storto 1999 et Müller et al. 2006).

Le deuxième chapitre se concentre sur la méthodologie utilisée pour la realisation des travaux sur le terrain. On discute l'importance de l'élicitation contrôlée de données pour une recherche en Linguistique Formelle (Matthewson 2004). On discute aussi quelques questions pratiques concernant les travaux sur le terrain, comme les négociations avec les membres de la communauté qui parle la langue étudiée et les principes éthiques applicables à la recherche en linguistique (Rice 2012).

Le troisième chapitre aborde les syntagmes verbaux en Karitiana. D'abord on donne un aperçu général sur la théorie de l'aspect lexical (Aktionsart) (Vendler 1957, Verkuyl 1996, Krifka 1998 et Rothstein 2004). Ensuite on présente une analyse de la télicité, la cumulativité, et l'atomicité des prédicats verbaux en Karitiana. Nous avons appliqué les tests classiques de la littérature linguistique sur la télicité, comme le paradoxe de l'imperfectif et les expressions comme pendant/en une heure. Nous avons aussi fait une analyse sur la cumulativité et l'atomicité en Karitiana. Les résultats obtenus sont les suivants: (i) Certains tests doivent être adaptés pour être apliqués en Karitiana (compte tenu des propriétés du temps, de l'aspect et des syntagmes nominaux de la langue); (ii) existe une différence entre prédicats téliques et atéliques en Karitiana; (iii) les prédicats vérbaux éventifs sont cumulatifs et comptables en Karitiana. 
Le quatriême chapitre est sur la modification de degré en Karitiana telle qu'elle est exprimée par l'adverbe pita(t) 'vrai/très/completement/beaucoup/vraiment'. On a constaté que pita $(t)$ peut apparaître dans le domaine nominal, adjectival et verbal. Son interprétation dépend du type d'échelle associeé au prédicat modifié. Quand pita(t) modifie des syntagmes nominaux, il signifie 'vrai' ou 'véritable'. Quand pita(t) modifie des syntagmes adjectivaux, il est interprété comme 'très' si l'adjectif possède une échelle ouverte, et 'completement' si l'adjectif possède une échelle fermée. Dans le domaine verbal, pita(t) a une restriction de distribution, il peut apparaître seulement dans des phrases avec des prédicats atéliques avec l'interpretation de degré. En revanche, quand on utilise pita(t) avec les prédicats téliques (accomplissements et achèvements), l'interpretation est quelque chose comme 'en effet', 'vraiment'.

Pour analyser ces caractéristiques atypiques de pita(t), on a utilisé le background théorique de la Sémantique des échelles et des degrés (Kennedy et McNally 2005, Kennedy 2007). Puis que les prédicats nominaux et verbaux n'ont pas une échelle lexicale à la différence des adjectives gradables, on propose qu'une fonction de degré Deg est responsable pour offrir un argument de degré et une échelle à la dénotation des noms et des verbes. L'échelle associée à des prédicats nominaux est une échelle fermée de précision et l'échelle associée à des syntagmes verbaux est une variable, qui est remplie selon le type du verbe. Les prédicats atéliques sont considerées comme des prédicats qui sont associés à une échelle ouverte et indeterminée alors que les prédicats téliques sont des prédicats associés à une échelle fermée et determinée.

Dans l'analyse que on propose pita(t) est un modificateur de degré qui manipule le degré associé au point de coupure de l'échelle representé par le degré $\mathrm{d}_{\mathrm{s}}$ en Keneddy (2007), qui est le degré normal quand l'échelle est ouverte, et le degré maximal quand l'échelle est fermée. La thèse soutient que considérer le point de coupure de l'échelle comme en degré disponible pour la modification et à considérer l'indétermination comme un trait aussi important pour la typologie des prédicats gradables.

Mots clés: modification; degré, adverbes, événements; langues indigènes. 


\begin{abstract}
SANCHEZ-MENDES, L. Degree Modification in Karitiana. 259pp. - Dissertation (PhD in Linguistics) - Universidade de São Paulo and Université Paris 8, 2014.
\end{abstract}

The aim of this dissertation is to present an anaysis of degree modification in Karitiana. Karitiana is a language of the Tupi family, Arikém branch, spoken by about 320 people on a demarcated area in the northwest of Brazil (Storto and Vander Velden 2005). This dissertation is divided in four chapters.

The first chapter is about the theoretical framework and about the basic properties of Karitiana grammar. The theoretical framework of this work is Formal Semantics. We present an introduction to this theoretical paradigm and to the tools used for semantic composition (Heim and Kratzer 1998). The part on Karitiana presents some of the basic characteristics of the language, like mood, tense, aspect, and the denotation of nominal phrases (Storto 1999, Müller et al. 2006).

The second chapter presents the methodology used in fieldwork. We discuss the importance of controled elicitation as a fieldwork methodology within a research in Formal Linguistics (Matthewson 2004). We also discuss some pratical issues of fieldwork, like the negociation with the comunity members and some ethical principles associated to a research in Linguistics (Rice 2012).

The third chapter is on the verbal phrases of Karitiana. First, we discuss the theories on lexical aspect (Aktionsart) (Vendler 1957, Verkuyl 1996, Krifka 1998 and Rothstein 2004). Then we present an analysis of telicity, cumulativity and atomicity of verbal phrases in Karitiana. We apply the classical tests found in the linguistic literature about telicity, like the imperfective paradox and expressions like for/in one hour. We also analyze cumulaticity and atomicity in Karitiana. The theses defended at the chapter are the following: (i) some of tests had to be adapted to be applied in Karitiana (considering the properties of tense, aspect and nominal phrases in the language); (ii) there is a difference between telic and atelic predicates in Karitiana; (iii) eventive verbal predicates are both cumulative and countable in the language.

The fourth chapter in on degree modification in Karitiana as performed by the adverb $\operatorname{pita}(t)$ 'true/very/completely/a lot/indeed'. Pita(t) can appear in the nominal, adjectival, and 
verbal domains. Its interpretation depends on the type of scale associated to the modified predicate. When pita(t) modifies Noun Phrases, it is interpreted as 'true'. They can be associated with a closed scale of precision. When pita(t) modifies Adjectival Phrases, it is interpreted as 'very' if the adjective has an open scale, and as 'completely' if the adjective has a closed scale. On the verbal domain, pita(t) has a restriction on its distribution. It can appear only with atelic predicates with a degree interpretation. On the other hand, when we use pita(t) with telic predicates (accomplishments and achievements), the interpretation is something like 'indeed'.

To analyze these atypical characteristics of pita(t), we assume a theoretical background based on a Scalar Semantics (Kennedy and McNally 2005, Kennedy 2007). Since nominal and verbal predicates don't have a lexical scale like the gradable adjectives we propose a Deg function that is responsible to provide a degree argument and a a scale to the denotation of nouns and verbs. The scale associated to nominal predicates is a closed scale of precision and the scale associated to Verb Phrases is a variable, filled according to the type of the verb. Atelic predicates are considered predicates that are associated to open and indetermined scales whereas telic predicates are associated to closed and determined scales.

The analysis we propose is that pita(t) is a degree modifier that manipulates the degree associated with the cutoff point of the scale represented by the degree $d_{s}$ in Kennedy (2007). That cutoff point is the standard degree when the scale is open and the maximal degree when the scale is closed. The thesis defended gives support for considering the cotoff point of a scale as an available degree for modification and for considering indeterminacy as an importanr feature of the typology of gradable predicates.

Key-words: modification, degree, adverbs, events, Indigenous Languages. 


\section{RÉSUMÉ DETAILLÉ}

\section{LA MODIFICATION DE DEGRÉ EN KARITIANA}

\section{Introduction}

Le but de cette thèse est de présenter une analyse de la modification de degré en Karitiana, telle qu'elle est exprimée par l'adverbe pita(t) 'beaucoup/ très/ vrai'. C'est la polysémie de cette expression, qui va de pair avec sa distribution catégorielle comme modificateur d'adjectifs, de verbes et de noms, qui a d'abord attiré notre attention sur le sujet. Les développements récents de la sémantique des degrés nous permettent d'en proposer une analyse unifiée, dans laquelle chaque sens est associé à un type de prédicat particulier défini par ses propriétés scalaires.

Le Karitiana est une langue de la famille Tupi, branche Arikém, parlée par environ 320 personnes dans une zone délimitée au nord-ouest du Brésil (Storto et Vander Velden 2005). Il s'agit d'une langue relativement bien décrite, dont la phonologie et la syntaxe ont fait l'objet d'un certain nombre de thèses et d'articles (Landin, D. 1983, 1984, 1988; Landin, R. 1982, 1987, 1989, Landin et Landin 1973, Everett, D. 1985, 1993, Storto 1994, 1996, 1998, 1999, 2001, 2002, 2003, 2005, 2008, 2010, 2011, 2012, Storto et Demolin 2005, Storto et Thomas 2012, Everett, C. 2006, Coutinho-Silva 2008, Rocha 2011a, 2011b, 2011c, 2012). En sémantique, des travaux récents portent sur l'aspect, le nombre nominal et verbal, la distributivité et la quantification adverbiale (Sanchez-Mendes 2006, 2008, 2009, 2011, 2012a, 2012b, Carvalho 2009, 2010, Müller, Storto et Coutinho-Silva 2006, Müller 2009, 2011, 2012, Müller et Negrão 2012, Müller et Sanchez-Mendes, 2008, 2009 e Sanchez-Mendes et Müller 2007). Notre étude sur la modification de degré vient compléter ce qu'on connaît de la sémantique du Karitiana, puisqu'elle clarifie les propriétés des syntagmes nominaux, verbaux et adverbiaux et leur relation potentielle avec les structures scalaires.

La thèse est divisée en quatre chapitres. Les premiers chapitres préparent le terrain pour l'analyse de la modification de degré, qui est présentée dans le chapitre 4. L'objectif du premier chapitre est de familiariser le lecteur avec la structure du Karitiana et avec le type de cadre sémantique dans lequel se fera l'analyse. Le deuxième chapitre explique la méthodologie qui nous semble la mieux adaptée à la description des langues peu étudiées, 
qu'on aborde par la travail de terrain, dans le cadre de la sémantique formelle. Le troisième chapitre éclaire des aspects de la sémantique des syntagmes verbaux en Karitiana qui auront une influence dans la description de pita(t).

\section{$2 \quad$ Karitiana}

Dans la première partie du premier chapitre, nous exposons un certain nombre de caractéristiques de la grammaire du Karitiana qui sont nécessaires pour la compréhension des exemples dans cette langue (Storto 1999 et Müller et al. 2006).

En Karitiana, l'ordre des mots dans phrases principales est SVO, comme le montre la phrase (1):

$$
\begin{array}{lll}
\text { Taso } & \varnothing \text {-na-oky-t } & \text { boroja. } \\
\text { homme } & 3 \text {-DECL-tuer-NFUt } & \text { serpent }
\end{array}
$$

'L'homme a tué la serpent'

(exemple 2 de Storto 1999, p. 125)

Les phrases subordonnées, par contre, ont l'ordre SOV ou OSV. Dans ces phrases, le verbe apparaît in situ sans marques de temps ni de personne.

$$
\begin{array}{llll}
\text { [Boroja taso oky tykiri ] } & \varnothing \text {-naka-hyryp-Ø õwã. } \\
\text { serpent homme tuer PERF } & \text { 3-DECL-pleurer-NFUT } & \text { enfant } \\
\text { 'Quand l'homme a tué la serpent, l'enfant a pleuré' } &
\end{array}
$$

(exemple 4 de Storto 1999, p. 125)

Le Karitiana est une langue ergative, dans laquelle le verbe s'accorde avec l'argument absolutif, c'est-à-dire, avec le sujet des verbes intransitifs et l'objet des verbes transitifs. 
(3)

Y-ta-opiso-t yn.
1s-DECL-entendre-NFUt je
'J'ai entendu'

(exemple 4 de Storto 1999, p. 161)

(4)

$$
\begin{aligned}
& \text { Yn a-ta-oky-j an. } \\
& \text { je 2s-DECL-tuer-Fut tu } \\
& \text { 'Je vais te tuer' }
\end{aligned}
$$

(exemple 1 de Storto 1999, p. 157)

Le Karitiana possède plusieurs morphèmes de mode pour la modalité phrastique (déclarative, assertive, citative, déontique, conditionnelle, et impérative cf. Storto 2002). Dans cette thèse, les exemples sont dans le mode déclaratif, qui est le mode par défaut de la langue. Le mode déclaratif est exprimé par les morphèmes $\{n a(k a)-\} /\{t a(k a)-\}$ préfixés aux racines verbales. Le morphème $\{n a(k a)-\}$ est utilisé quand l'accord est avec la troisième personne, alors que $\{t a(k a)-\}$ est utilisé dans tous les autres cas. La syllabe $\{-k a\}$ est un morphème augmentatif qui apparaît quand la première syllabe du verbe est accentuée.

$$
\begin{aligned}
& \text { Y-taka-tat-Ø. } \\
& \text { 1s-DECL-aller-NFut } \\
& \text { 'J('y) suis allé' }
\end{aligned}
$$

(exemple 5 de Storto 2008, p. 186)

(6)

$$
\begin{array}{lll}
\text { Yn } & \varnothing \text {-na-oky-t } & \text { pikom. } \\
\text { je } \quad 3 \text {-DECL-tuer-NFut } & \text { singe } \\
\text { 'J'ai tué le singe' } &
\end{array}
$$

(exemple 6 de Storto 2008, p. 187)

En Karitiana, les morphèmes de temps sont le futur et le non-futur (Storto 2002). Dans les phrases déclaratives, le suffixe du non-futur est $\{-t\}$, lorsque le verbe se termine par une voyelle, et $\{-\varnothing\}$ s'il se termine par une consonne. 
(7)

$\begin{array}{lll}\text { Taso } & \varnothing \text {-naka-'y-t } & \text { kinda'o. } \\ \text { homme } & 3 \text {-DECL-manger-NFut } & \text { fruit }\end{array}$

'L'homme mange / a mangé des fruits'

(8) João Ø-na-pimbik-Ø gooj.

João 3-DECL-pousser-NFUT voiture

'João pousse / a poussé la voiture'

Le suffixe de futur est $\{-j\}$, quand le verbe se termine par une voyelle, et $\{-i\}$ s'il se termine par une consonne.

(9) Taso Ø-na-oky-j boroja.

homme 3-DECL-tuer-FUT serpent

'L'homme va tuer le serpent'

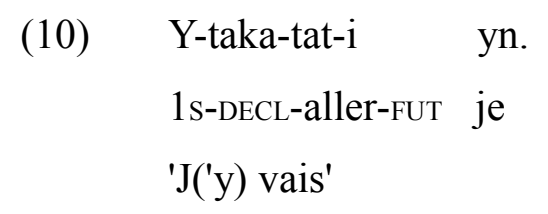

(exemple 12.70 de Everett, C. 2006, p. 269)

Le Karitiana possède plusieurs expressions pour déterminer l'aspect (Storto 2002). C'est l'aspect imperfectif qui a été le mieux décrit dans la littérature sur cette langue. Les morphèmes d'aspect en Karitiana sont sémantiquement complexes. Outre l'expression de l'imperfectivité, ils manifestent des notions qui concernent la posture du corps et le nombre grammatical du sujet. Les phrases (11), (12) et (13) exemplifient les morphèmes tyja, tyso et tysyp et l'information sur la posture du sujet qu'ils véhiculent.

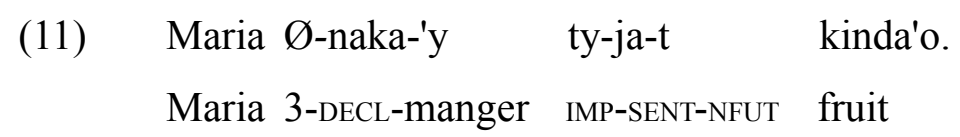

'Maria est en train de manger des fruits (assise)'

(exemple 40b de Carvalho 2010, p. 46) 
(12)
Maria Ø-naka-'y ty-so-t kinda'o.
Maria 3-DECL-manger IMP-EM.PÉ-NFUT fruit
'Maria est en train de manger des fruits (debout)'

(exemple 40c de Carvalho 2010, p. 46)

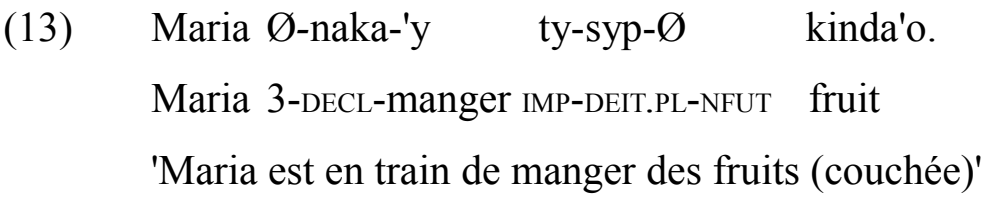

(exemple 40d de Carvalho 2010, p. 46)

Les phrases (14) et (15) illustrent la restriction de nombre grammatical de deux morphèmes aspectuels. Le morphème tysyp ne peut être utilisé que dans des contextes de sujet pluriel et tykat est exclusif des contextes de sujet singulier.

$\begin{array}{lll}\text { Ombaky i-pyt'y } & \text { ty-syp- } \varnothing \\ \text { jaguar } & \text { PART-manger } & \text { IMP-DEIT.PL-NFUT }\end{array}$

'Les jaguars sont en train de manger (couchés)'

(exemple 12.89 de Everett, C. 2006, p. 278)

$$
\begin{aligned}
& \text { Ø-na-ohit ty-ka-t õwã. } \\
& \text { 3-DECL-pêcher IMP-MOv.SG-NFUT enfant } \\
& \text { 'L'enfant est en train de pêcher (assis)" }
\end{aligned}
$$

Une construction très fréquente dans le domaine verbal est la construction copulative. La construction peut contenir comme prédicat des substantifs (16), des adjectifs (17), et des verbes intransitifs (18).

$\begin{array}{ll}\text { Byyty } \varnothing \text {-na-aka-t } & \text { kinda'o-t. } \\ \text { papaye } 3 \text {-DECL-COP-NFUT } & \text { fruit-ABS } \\ \text { 'Le papaye est une fruit' } & \end{array}$

(exemple 1 de Storto 2010, p. 2) 
(17)
Taso Ø-na-aka-t i-se'a-t.
homme 3-DECL-COP-NFUT PART-bon-ABS

'L'homme est bon'

(exemple 5 de Storto 2010, p. 2)

(18)
Taso
Ø-na-aka-t
i-kat-t.
homme 3-DECL-COP-NFUT
PART-dormir-ABS

'L'homme a dormi'

(exemple 7 de Storto 2010, p. 2)

Les verbes transitifs, par contre, ne peuvent pas apparaître dans la construction copulative.
* Taso
Ø-na-aka-t
i-'y-t
(ta-ti'y).
homme 3-DECL-COP-NFUT
PART-manger-ABS (3ANAF-nourriture)

'L'homme a mangé (sa nourriture)'

(exemple 10 de Storto 2010, p. 3)

Storto (2010) analyse les construction copulatives comme des phases qui contiennent deux propositions grammaticales. La copule naakat sélectionne une petite proposition (small clause) nominalisée. Le sujet de la small clause monte à la position qui précède la copule. Le suffixe $\{-t\}$ ou $\{-\varnothing\}$ qui apparait dans la small clause est une marque morphosyntaxique du mouvement du sujet. La structure des constructions copulatives est la suivante:

Sujet $_{\mathrm{i}}$ naakat [nom $\left[\mathrm{sc} \mathrm{t}_{\mathrm{i}} \mathrm{X}\right]$ ]

$X$ peut être un substantif, un adjectif, ou un verbe intransitif

(représentation 13a de Storto 2010, p. 3)

Pour ce qui est du domaine nominal, les syntagmes nominaux du Karitiana ont une particularité. Ils sont toujours des syntagmes nus, dans le sens où ils n'ont pas de matériel fonctionnel dans la position de déterminant. Les noms de la langue n'ont pas de marques de nombre ni classificateurs (Müller, Storto et Coutinho-Silva 2006). Ils sont utilisés librement 
sans aucun morphème fonctionnel comme arguments dans la phrase (Müller, Storto et Coutinho-Silva, 2006, Müller 2009). L'exemple (21) montre que les noms communs qui dénotent des ensembles d'individus, comme jonso 'femme', ou qui désignent une substance, comme ese 'eau', qui se comportent respectivement comme des noms comptables et massifs dans les langues du monde, apparaissent nus sans flexion de nombre ou quantificateurs nominaux. Les syntagmes nominaux nus manifestent ce que l'on s'accorde à appeler le nombre neutre, qui est indifférencié par rapport au singulier ou pluriel. Un prédicat au nombre neutre dénote aussi bien des atomes que les sommes obtenues à partir de ces atomes.

Jonso Ø-naka-ot-Ø ese.

femme 3-DECL-prendre-NFUT eau

'Une/des femme(s) ont pris de l'eau'

Pour résumer, le Karitiana est une langue ergative dont l'ordre des constituants la plus répandue est SVO. Concernant les morphèmes verbaux, sa modalité phrastique par défaut est le mode déclaratif, ses morphèmes de temps sont le futur et le non-futur, et elle possède des marques d'aspect imperfectif qui expriment aussi des informations sur la posture du corps et le nombre singulier ou pluriel du sujet. En Karitiana, la construction copulative est une construction très répandue, qui apparaîtra dans beaucoup des exemples de cette étude. Enfin, les syntagmes nominaux nus ont une dénotation de nombre neutre. Ces caractéristiques contribuent à la compréhension des exemples fournis, et certaines d'entre elles ont une influence directe sur l'analyse proposée, comme le temps et l'aspect et les propriétés sémantiques des syntagmes nominaux.

\section{Sémantique Formelle}

Dans la deuxième partie du chapitre d'introduction, nous présentons l'approche théorique adoptée dans la thèse, celle de la sémantique formelle. Nous nous attardons sur l'objet de l'analyse sémantique dans ce paradigme et sur les outils employés pour la composition sémantique (Heim et Kratzer 1998). Dans le cadre de la sémantique formelle, le sens d'une phrase est déterminé par ses conditions de vérité. Il s'agit donc d'une théorie 
vériconditionnelle, qui associe à une phrase ses conditions de vérité dans une formulation comme (22):

La phrase “__ " est vrai si et seulement si

En (22), la partie entre guillemets est la phrase de la langue objet et la partie après $s i$ et seulement si est le sens de la phrase décrit dans un métalangage. L'équivalence formulée en (22) doit être le résultat d'une analyse sémantique qui attribue à chaque composante de la phrase une valeur sémantique. La tâche du chercheur consiste à découvrir la façon dont le système sémantique produit le sens d'une phrase à partir de la valeur sémantique de ces composantes et de la façon dont elles sont combinées.

L'interprétation sémantique compositionnelle comprend deux éléments fondamentaux: un lexique et un ensemble de règles qui permettent de calculer la valeur sémantique des combinaisons d'éléments. La valeur sémantique d'une expression quelconque est sa dénotation, un objet formel dans un modèle du "monde" ou de la réalité. Par convention, on représente la dénotation d'une expression en enfermant cette dernière entre crochets doubles [I \. Dans les approches actuelles en sémantique compositionnelle, on considère qu'il y a une seule règle fondamentale pour la composition sémantique, l'application fonctionnelle. C'est une règle qui consiste en appliquer une fonction donnée à un argument. On considère que toute combinaison syntaxique est binaire, et que l'un des éléments de la combinaison est la fonction et l'autre l'argument de cette fonction. Dans le cas le plus simple, nous avons la combinaison d'une expression saturée, qui dénote un individu, avec une expression non-saturée, qui requiert un complément et dénote une fonction qui prendra cet individu comme argument (Frege 1892). La notation lambda permet de représenter les fonctions en liant par lambda l'argument auquel la fonction doit s'appliquer. En (23) nous donnons un exemple simplifié d'interprétation sémantique compositionnelle de la phrase Inácio travaille : 

a. [Inácio ] = Inácio
Lexique
b. $\llbracket$ travaille $\rrbracket=\lambda \mathrm{x}$. travaille $(\mathrm{x})$
Lexique
c. $\llbracket$ Inácio travaille $\rrbracket=\llbracket$ travaille $\rrbracket(\llbracket$ Inácio $\rrbracket)$
Applic. Fonction.
d. $\quad$ Inácio travaille $\rrbracket=[\lambda \mathrm{x}$. travaille $(\mathrm{x})]$ (Inácio)

Remplacement

e. 【Inácio travaille $\rrbracket=1$ si et seulement si travaille (Inácio)

Réduction du lambda-terme

f. "Inácio travaille" est vrai si et seulement si travaille (Inácio)

Dans la représentation (22)

L'interprétation compositionnelle aboutit ici à une équivalence entre la phrase originale et ses conditions de vérité exprimées dans le langage de la logique des prédicats. L'application fonctionnelle exige qu'il y ait une compatibilité entre les termes, dans le sens où l'expression qui joue le rôle d'argument doit être compatible avec le type d'argument requis par l'expression qui joue le rôle de fonction. Cette compatibilité est définie au moyen des types sémantiques. C'est pour cette raison qu'on dit que l'interprétation sémantique est déterminée par les types sémantiques (type-driven interpretation).

Les deux types de base sont le type e pour les individus, auquel correspondent p. ex, les noms propres comme Inácio, et le type t pour les valeurs de vérité, qui est le type des phrases. Ces sont les deux types correspondant aux expressions saturées. Les types des expressions non-saturées sont formés par la combinaison de ces deux types de base. Ce sont des représentations de fonctions binaires dont le premier élément donne le type d'argument et le deuxième élément le type des valeurs de la fonction. Un verbe intransitif comme travaille a un type composé: $<\mathrm{e}, \mathrm{t}\rangle$. Cela signifie que travaille est une fonction qui prend un argument de type $\mathbf{e}$, le type de individus, et retourne une valeur de type $\mathbf{t}$, c'est à dire, une valeur de vérité. Les dénotations dans (24) présentent moyennant des indices les types de chaque élément entrant dans la composition décrite ci-dessus.
a. [[ Inácio $]]_{\mathrm{e}}=$ Inácio
b. $\quad[$ travaille $]]_{<, \triangleright, \triangleright}=\lambda \mathrm{x}_{\mathrm{e}}$. travaille $(\mathrm{x})$
c. 【Inácio travaille $\rrbracket_{\mathrm{t}}=\llbracket$ travaille $\rrbracket_{<, \triangleright}\left(\llbracket\right.$ Inácio $\left.\rrbracket_{\mathrm{e}}\right)$
d. $\quad[\text { Inácio travaille }]_{\mathrm{t}}=1$ si et seulement si travaille (Inácio) 


\subsection{Sémantique d'événements}

Une innovation importante dans l'histoire récente de la sémantique formelle a amené à introduire dans l'ontologie une sorte d'objet distincte des individus, les événements, et avec eux, un type/sorte simple ultérieur. En effet, dans la sémantique d'événements on maintient que les verbes ne peuvent pas être considérés uniquement comme des fonctions des individus à des valeurs de vérité, mais que ce sont des fonctions qui prennent aussi comme arguments des événements (Davidson 1967 et Parsons 1990). Parsons (1990) considère que les verbes sont des prédicats des événements, c'est à dire, les événements sont les seuls arguments des verbes et leurs arguments nominaux sont insérés dans la forme logique via des prédications secondaires, représentées par les rôles thématiques. L'exemple (25) illustre la dénotation d'un verbe transitif, blesser dans la proposition de Parsons (1990).

$$
[[\text { blesser }]]=\lambda \mathrm{x} \cdot \lambda \mathrm{y} \cdot \lambda \mathrm{e} \cdot \operatorname{blesser}(\mathrm{e}) \& \operatorname{Agent}(\mathrm{y})(\mathrm{e}) \& \text { Thème }(\mathrm{x})(\mathrm{e})^{1}
$$

La perspective de la sémantique compositionnelle sera adoptée dans cette thèse et nous amènera à proposer que pita(t) entre dans la composition comme une fonction qui prend des prédicats gradables comme arguments. Nous adoptons la sémantique d'événements, parce qu'elle représente plus adéquatement la sémantique des verbes. Cela nous permet d'attribuer deux représentations différentes aux deux formes que présente pita(t). Nous soutenons que pitat, avec un suffixe $\{-t\}$, sélectionne des prédicats gradables d'événement, alors que pita sélectionne des prédicats gradables qui ne contiennent pas de variable d'événement. Le morphème $\{-t\}$ ajoute un argument d'événement dans la dénotation de pita.

\section{$4 \quad$ Méthodologie des Travaux de Terrain}

Le deuxième chapitre se concentre sur la méthodologie utilisée dans les travaux de terrain pour la collecte de données. Étant donné l'objectif de cette thèse, celui d'analyser la modification de degré, nous avons dû adapter cette méthodologie pour recueillir des phrases et des contextes qui soient à même d'éclairer la contribution sémantique de l'adverbe pita(t) et la relation de cet adverbe avec les échelles qui peuvent être associées aux prédicats modifiés.

On adopte la notation traditionnelle d'utilisation des variables $\mathbf{x}$ et $\mathbf{y}$ pour les objets et $\mathbf{e}$ pour les événements. 
Le travail de terrain en linguistique, dans son sens traditionnel, impliquait un chercheur vivant dans la communauté, qui parlait la langue à étudier. Les linguistes comme Sapir et Bloomfield ont fondé la tradition structuraliste de travail sur le terrain, dans laquelle la collecte de données ne se distingue pas de l'interaction et de l'observation directe des communautés étudiées. Dans cette tradition, tout la linguistique descriptive découlait du travail sur le terrain et de l'interaction avec des locuteurs natifs (Chelliah e de Reuse 2011).

Cependant, les linguistes post-structuralistes reconnaissent qu'il est possible de faire un travail sur le terrain avec beaucoup de succès grâce à la collecte de données basée sur des entretiens d'enquête, sans qu'il soit nécessaire que le linguiste devienne un membre de la communauté qui parle la langue étudiée. Selon Chelliah et de Reuse (2011), il n'existe pas de différence qualitative entre ces deux modes de collecte. Il est possible de faire du bon travail de terrain au moyen d'entretiens ciblés, et l'immersion dans la langue étudiée ne garantit pas la qualité du travail de terrain. Ces auteurs affirment que le travail de terrain en linguistique dans des environnements non exotiques est plus fréquent qu'il ne paraît à la lecture des textes sur le sujet. En ce sens, le linguiste de terrain n'est pas nécessairement la caricature du chercheur qui dompte la jungle à la recherche de données linguistiques.

Dans ce chapitre, nous essayons de montrer comment le choix du cadre théorique d'une recherche en linguistique détermine la méthodologie utilisée pour la collecte des données. Nous partons de l'idée qu'il n'y a pas de différence qualitative entre la description et l'analyse linguistique. La recherche descriptive ne peut être effectuée sans une théorie et celle qui est considérée théorique ne peut pas se développer sans l'utilisation d'un corpus. Par conséquent, la procédure de collecte de données utilisée dans cette recherche est fortement liée au paradigme théorique de la sémantique vériconditionnelle. Pour recueillir des phrases accompagnés de leurs conditions de vérité, l'objet d'étude du paradigme utilisé, l'élicitation contrôlée est la méthode la plus appropriée (Matthewson 2004).

\subsection{L'élicitation Contrôlée}

L'élicitation contrôlée est une méthode de collecte de données basée sur des entretiens qui a essentiellement deux étapes: les traductions et les jugements des contextes. La première étape de l'élicitation contrôlée implique la collecte de traductions, qui sont des outils importants du travail sur le terrain. Cependant, elles doivent être utilisées comme des indices, 
et non comme des résultats: elles constituent la toute première étape du travail sur le terrain. Par exemple, en demandant la traduction en Karitiana pour la phrase Inácio a beaucoup attendu on a pu éliciter la phrase suivante:

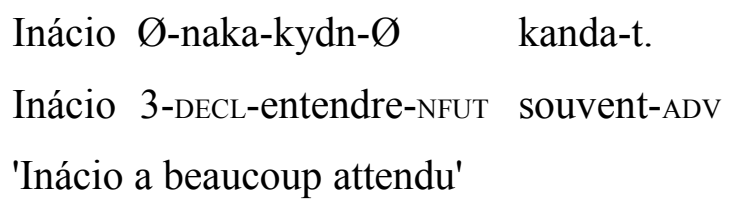

En Karitiana, la phrase (26) ne peut être utilisée que dans une situation dans laquelle Inácio a attendu beaucoup de fois. Elle n'est pas appropriée dans une situation où Inácio a attendu une fois pendant longtemps. Il s'ensuit que kanda(t) n'est pas sémantiquement équivalent à beaucoup, mais il se comporte plutôt comme l'adverbe souvent en français. La différence entre l'itération et la durée est très importante pour l'analyse des adverbes de quantification (Doetjes 2007). La tentative de traduction de la phrase (26) montre que la méthode de traduction toute seule n'est pas suffisante, parce qu'elle peut cacher des informations que sont très pertinentes pour l'analyse sémantique.

Ainsi, la deuxième étape de élicitation contrôlée suggérée par Matthewson (2004) est cruciale pour les études qui examinent les conditions de vérité des phrases. Il s'agit de la collecte des jugements de valeur de vérité des phrases dans des contextes particuliers. Par contexte nous entendons ici les circonstances dans le monde par rapport auxquelles les phrases devraient être adéquates.

La relation de la phrase avec le contexte peut être de deux types:

(27) $\quad$ Si un locuteur accepte la phrase $\mathrm{S}$ dans un contexte $\mathrm{C}, \mathrm{S}$ est vrai en $\mathrm{C}$.
b. $\quad$ Si une phrase S est fausse dans un contexte $\mathrm{C}$, le locuteur rejette S dans C.

Il faut souligner que, si le consultant rejette une phrase dans un contexte, ceci n'est pas suffisant pour supposer que la phrase est fausse. Parfois, une phrase est vraie, mais elle n'est pas adéquate ou heureuse dans ce contexte. L'infélicité d'une phrase peut être associée à des défaillances pragmatiques.

Dans la première partie de ce chapitre, nous évoquons aussi un certain nombre de questions pratiques concernant les travaux sur le terrain, comme les négociations avec les 
membres de la communauté qui parle la langue étudiée et les principes éthiques applicables à la recherche en linguistique (Rice 2012). Ces aspects sont importants car ils sont souvent l'objet de discussions parmi les linguistes qui étudient des langues indigènes. Dans le cas de la recherche sur le Karitiana, la négociation avec la communauté a été un élément fondamental de la recherche. Nous avons fait une assemblée avec les membres de la communauté en 2008, dans le but de présenter la recherche sur la langue qui était réalisée à l'époque. En 2013, une autre réunion a été faite pour présenter les résultats et renégocier le partenariat entre les linguistes et les membres de la communauté.

\subsection{Méthodologie et Questions Pragmatiques}

La deuxième partie de ce chapitre sur la méthodologie examine un certain nombre de questions pragmatiques impliquées dans l'analyse sémantique de la quantification adverbiale en Karitiana. Plus précisément, notre intention est de montrer comment des inférences pragmatiques peuvent aider à analyser correctement le sens des adverbes comme kanda(t) dans des phrases comme (28).

$$
\begin{array}{llll}
\text { jonso } & \varnothing \text {-naka-ot- } \varnothing \quad \text { kanda-t } & \text { ese. } \\
\text { femme } & \text { 3-DECL-prendre-NFut } & \text { souvent-ADV } & \text { eau } \\
\text { 'Des femmes ont souvent pris de l'eau' } &
\end{array}
$$

Les contextes dans lesquels la phrase est jugée appropriée sont:
a. 'Beaucoup de femmes ont pris de l'eau'
b. 'Des femmes ont pris beaucoup d'eau'
c. 'Des femmes ont souvent pris de l'eau'
d. 'Beaucoup de femmes ont souvent pris beaucoup d'eau'

On observe dans (29) que les noms communs dans des phrases avec kanda(t) ont deux interprétations possibles: (i) une interprétation faible, de quantité indéterminée (29c); et (ii) une interprétation forte, de beaucoup de femmes et beaucoup d'eau (29a), (29b), et (29d). 
Nous avons adopté une proposition gricéenne d'analyse du sens, en essayant de ne pas multiplier les significations. Nous proposons que kanda(t) est un quantificateur adverbial qui porte exclusivement sur les événements et qui signifie toujours 'plusieurs fois'. Dans notre analyse, les interprétations 'beaucoup de femmes' et 'beaucoup d'eau', dans lesquelles kanda(t) semble quantifier aussi sur les arguments, son dérivées par inférence pragmatique. L'argument principal en faveur de cette proposition est basée sur des contextes inappropriés pour la phrase (28), décrits en (30):
a. 'Beaucoup de femmes ont pris de l'eau une fois'
b. $\quad$ 'Des femmes ont pris beaucoup d'eau une fois'

En outre, la phrase (28) peut être utilisé dans des contextes où une seule femme participe du événement, et il y a une petite quantité d'eau, comme indiqué dans les contextes décrits dans (31):
a. 'Une femme a souvent pris de l'eau'
b. 'Des femmes ont souvent pris un peu d'eau'

Le contraste entre les contextes décrits dans (29), (30) et (31) montre que la référence à de nombreuses entités est une possibilité dans les phrases avec kanda(t) (contextes 29a à 29d), mais pas une nécessité (contextes $31 \mathrm{a}$ et $31 \mathrm{~b}$ ), alors que la lecture de plusieurs événements est une exigence (contextes 30a et 30b).

Cela indique que kanda(t) quantifie toujours sur des événements. Les interprétations possibles 'beaucoup de femmes' et de 'beaucoup d'eau' surgissent parce que les syntagmes nominaux dans la langue ont un nombre neutre. Ces interprétations sont fournies par des inférences pragmatiques, ce qui signifie qu'elles peuvent être déduites, dans certains contextes, mais ne font pas partie des conditions de vérité de la phrase. La dénotation de la phrase (28) est donnée dans (32):

$$
\llbracket \text { jonso nakaot kandat ese } \rrbracket=1 \text { sse } \exists \mathrm{E} . \forall \mathrm{e} \in \mathrm{E}[\operatorname{prendre(y)(e)~\& ~eau~(y)~\& ~}
$$

$$
\operatorname{Agent}(\mathrm{x})(\mathrm{e}) \& \text { femme }(\mathrm{x}) \&|\mathrm{E}|>\mathrm{N}]^{2}
$$

\footnotetext{
2 Ici nous adoptons la proposition de Kratzer (1996) selon laquelle l'argument interne est le seul vrai argument du verbe. L'argument externe est syntaxiquement inséré par un syntagme Voix qui introduit le rôle
} 
"Il existe un ensemble d'événements tel que chaque événement qui est un membre de cet ensemble est un événement de prendre de l'eau, dont l'agent est une femme et que la cardinalité de l'ensemble est supérieure à une cardinalité contextuellement donnée"

Compte tenu de l'analyse présentée, son développement ultérieur peut prendre des chemins différents. Le premier consisterait à enquêter sur les interprétations privilégiées avec kanda $(t)$. Les travaux de pragmatique discutent généralement les contextes privilégiés qui peuvent contribuer à engendrer des inférences. Ce type d'enquête avec des phrases contenant kanda(t) reste un travail pour la recherche future, compte tenu de la difficulté de faire une recherche de ce genre dans une langue partiellement décrite dont l'enquêteur n'est pas un locuteur natif.

Un deuxième chemin à explorer est celui de déterminer comment la différence entre les interprétations disponibles et annulables interfère avec la méthodologie de travail sur le terrain pour recueillir des données de la langue. La méthodologie suggérée pas Mathewson (2004) a une étape qui propose:

Si un locuteur accepte la phrase S dans un contexte C, S est vrai en $\mathrm{C} . \quad=(27 \mathrm{a})$

Pourtant, cela ne garantit pas que le contexte proposé et accepté soit le seul contexte qui peut représenter les conditions de vérité de la phrase, c'est à dire son sens (pour le paradigme théorique adopté dans cette thèse). Il est possible que le contexte proposé soit plus riche, dans le sens où il pourrait inclure plus d'information que les conditions de vérité de la phrase. La phrase en français ci-dessous illustre un cas où il n'y a pas de correspondance exacte entre le contexte et les conditions de vérité, alors qu'un locuteur serait amené à accepter la phrase dans le contexte.

Il y a des enfants dans la piscine.

Contexte d'élicitation :

thématique d'agent. 
En ce moment, il y a beaucoup d'enfants dans la piscine. Puis-je utiliser la phrase Il y a des enfants dans la piscine?

La phrase (34) est vraie dans le contexte (35), mais elle véhicule moins d'information que le scénario décrit dans le contexte. La phrase serait également vraie dans un contexte où il n'y a que peu d'enfants. Pour cette raison, nous proposons d'ajouter une étape supplémentaire au protocole de Matthewson (2004). Il est nécessaire d'éliciter les scénarios limites pour obtenir le contexte minimal qui représente les conditions de vérité de la phrase. Les scénarios limites sont ceux qui peuvent aider à définir les informations contenues dans le sens de la phrase. Autrement dit, ce sont ceux qui tentent d'expliciter ce qui fait vraiment partie des conditions de vérité d'une phrase (sa sémantique) et ce qui est un sens dérivé de son utilisation (pragmatique). Par exemple, les contextes limites pour déterminer le sens de la phrase (28) avec l'adverbe kanda(t) seraient les suivants :

a. Il y a une seule femme qui est allée à la rivière pour chercher de l'eau, est-ce que je peux utiliser la phrase jonso nakaot kandat ese?

b. Il y a une femme qui est allée à la rivière pour chercher de l'eau, elle y est allée beaucoup de fois, mais elle a pris seulement un peu d'eau, est-ce que je peux utiliser la phrase jonso nakaot kandat ese?

Le contexte (36a) vise à évaluer si l'interprétation "beaucoup de femmes" dans les phrases avec kanda(t) est une exigence, c.-à-d. si elle fait partie du sens de la phrase, ou si elle est dérivée de façon pragmatique dans certains contextes. La réponse obtenue à la question (36a) a été 'oui', ce qui indique que le syntagme jonso dans la phrase jonso nakaot kandat ese a une dénotation de nombre neutre et peut indiquer une, quelques ou même de nombreuses femmes. Le contexte (36b) a le même objectif, mais à l'égard du thème ese 'eau'. La réponse obtenue a été également 'oui', confirmant l'hypothèse que l'adverbe kanda(t) quantifie seulement sur les événements de la phrase. Il n'a pas de effets sémantiques sur les syntagmes nominaux argumentaux de la phrase.

En résumé, ce chapitre traite des questions liées au travail de terrain et présente la méthodologie de collecte de données utilisées dans une recherche en sémantique formelle. Il a 
pour fonction de clarifier la façon dont nous avons obtenu les données utilisées dans cette thèse et la raison pour laquelle nos exemples sont souvent accompagnés d'une description des situations dans lesquelles ils sont jugés appropriés ou inappropriés.

\section{$5 \quad$ Syntagmes Verbaux}

Le troisième chapitre aborde la sémantique des syntagmes verbaux en Karitiana. Il sert à décrire la sémantique des prédicats verbaux dans la langue. La classification des prédicats verbaux selon leur aspect lexical, et plus particulièrement la distinction téliqueatélique, sera cruciale pour comprendre comment fonctionne la modification de degré dans le domaine verbal.

Nous donnons d'abord un aperçu général de la théorie de l'aspect lexical (Aktionsart) (Vendler 1957, Verkuyl 1996, Krifka 1998 et Rothstein 2004). Vendler (1957) a été le premier auteur à systématiser une classification du lexique verbal qui distingue les prédicats d'état, d'activité, d'accomplissement, et d'achèvement. La première distinction proposée par Vendler (1957) est celle entre prédicats téliques et atéliques. Des syntagmes comme dessiner un cercle sont téliques parce que ils ont une fin déterminée. Leur sens comporte un seuil du procès tel que, une fois ce seuil atteint, le procès ne saurait pas continuer, et tel qu'on ne peut affirmer que le procès ait eu lieu si ce seuil n'a pas été atteint. Courir, par contre, est un prédicat atélique, puisqu' il n'a pas une fin déterminée lexicalement. Pour cette raison, c'est possible de dire finir de dessiner un cercle, mais on ne peut pas dire finir de courir. Dessiner un cercle est un prédicat d'accomplissement et courir est une activité. Les deux sont des prédicats duratifs. En revanche, les prédicats d'achèvement, comme reconnaître, et d'état, comme aimer, ne comportent pas de progression temporelle, ni de stades de développement. Reconnaître dénote un changement d'état instantané, une transition entre deux états contradictoires, et est classifié pour cette raison comme télique. Quant aux verbes d'état, ils sont vrais ou faux à un instant donné par rapport à une situation, mais ils ne présentent pas de variation ni de progression dans le temps. Ils sont atéliques comme les activités.

Verkuyl (1996) et Krifka (1998) proposent une extension de la caractérisation de la classification vendlérienne qui tient compte des propriétés de tout le syntagme verbal. La motivation principale pour considérer l'aspect lexical comme un phénomène déterminé au niveau du syntagme verbal est la nature de l'objet, qui peut avoir une influence sur le 
comportement du prédicat par rapport aux critères de classification. Ainsi, p. ex., le verbe manger décrit un événement télique quand il se construit avec un objet délimité de type une pomme, mais il est atélique lorsqu'il est utilisé avec un objet non-délimité, comme des pommes.
a. Jean a mangé une pomme.
TÉLIQUE
b. Jean a mangé des pommes.
ATÉLIQUE

Selon Rothstein (2004), la télicité est une conséquence de l'atomicité. Sa proposition générale est que tout le domaine verbal est grammaticalement comptable, et contient en conséquence des unités discrètes et disjointes, des atomes. Selon l'auteur, les verbes dénotent des fonctions du type:

$$
\llbracket \mathrm{V} \rrbracket=\lambda \mathrm{e} \cdot \mathrm{P}(\mathrm{e}) \& \operatorname{MEAS}(\mathrm{e})=<1, \mathrm{U}>
$$

(représentation 21 de Rothstein 2008, p.13)

Ces sont des prédicats d'événements associés à une mesure qui donne la cardinalité 1 (celle des atomes) à chaque instanciation du prédicat. La différence entre les prédicats téliques et atéliques réside, d'après Rothstein, dans les critères de l'individuation des événements atomiques. Lorsque les critères d'individuation (U) sont lexicalement spécifiés, le prédicat est télique. Si ils ne sont pas lexicalement spécifiés le prédicat est atélique. Cela veut dire que les critères d'individuation pour les prédicats téliques sont indépendants du contexte et ils se maintiennent constants dans tout contexte, alors que ce qui compte comme un atome pour un prédicat atélique varie selon les contextes. Les exemples suivants illustrent cette différence.

$$
\begin{aligned}
& \text { a. } \llbracket \text { reconnaître } \rrbracket=\lambda \mathrm{e} . \mathrm{P}(\mathrm{e}) \wedge \operatorname{MEAS}(\mathrm{e})=<1 \text {,reconnu }> \\
& \text { b. } \llbracket \text { courir } \rrbracket=\lambda \mathrm{e} . \mathrm{P}(\mathrm{e}) \wedge \operatorname{MEAS}(\mathrm{e})=<1, \mathrm{U}>
\end{aligned}
$$

Au delà de la distinction entre état, activité, accomplissement, et achèvement, Carlson (1980) et Kratzer (1995) proposent une distinction entre prédicats stage-level et individual-level. Carlson (1980) introduit une distinction entre les propriétés transitoires des individus qui sont susceptibles de se répéter dans le temps pour un même individu - propriétés 
stage-level - et les propriétés qui sont considérés permanentes et ne peuvent pas se répéter pour le même individu à des moments différents de son histoire - propriétés individual-level. Ces propriétés sont exprimées respectivement par les prédicats stage-level et individual-level. Les prédicats stage-level sont, par exemple, être heureux, vouloir, être disponible, etc. Des exemples de prédicats individual-level sont croire en Dieu, aimer, savoir, être brésilien.

\subsection{Les Classes Verbales en Karitiana}

Nous avons essayé de déterminer le comportement des prédicats du Karitiana par rapport à la propriété de télicité en appliquant les tests classiques de la littérature linguistique (Dowty 1979, Parsons 1990, Chierchia 2003). La difficulté principale que nous avons rencontrée a été celle de trouver un test fiable pour déterminer les classes de verbes en Karitiana. Nous avions en effet l'intuition que le comportement de pita(t) était sensible à la classe aspectuelle du prédicat. Nous avons pu confirmer cette intuition, de façon telle que la division des prédicats verbaux en fonction de leur aspect lexical sera cruciale pour analyser la modification de degré dans le chapitre suivant.

Le résultat obtenu est que la distinction entre des prédicats téliques et atéliques existe bel et bien en Karitiana, mais les tests doivent être adaptés et soigneusement choisis en prenant compte des particularités des syntagmes nominaux et de l'aspect dans cette langue.

Le fait qu'en Karitiana les syntagmes nominaux formés par des noms communs ont le nombre neutre entraîne des conséquences pour détecter la télicité dans cette langue. Étant donné que les syntagmes nominaux nus ont une dénotation cumulative, les verbes de type incrémental comme manger et construire semblent être toujours atéliques avec ces syntagmes comme arguments.

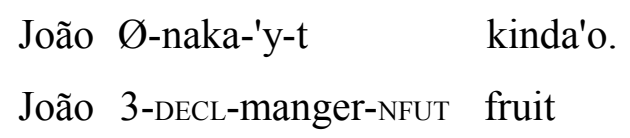

'João a mangé des fruits (une ou plus d'une)'

$$
\begin{aligned}
& \text { João i-kokot- } \quad \text { pa-ty. } \\
& \text { João PART-traverser-ABS rue-OBL } \\
& \text { 'João a traversé des rue (une ou plus d'une)' }
\end{aligned}
$$


Ceci n'a rien d'étonnant, parce que nous savons que la télicité ne se manifeste que lorsque l'objet lui même est délimité ou quantique. La stratégie suivie pour obtenir des arguments nominaux qui permettent à la télicité de se manifester en Karitiana a été d'utiliser des noms propres ou des noms modifiés par des numéraux cardinaux. Les exemples (42) et (43) montrent comment on peut obtenir des lectures téliques pour les prédicats d'événement évoqués en (40) et (41) moyennant ces stratégies.

$$
\begin{array}{lll}
\text { João } Ø \text {-naka-'y-t } & \text { myhin-t } & \text { kinda'o. } \\
\text { João 3-DECL-manger-NFUt } & \text { un-OBL } & \text { fruit } \\
\text { 'João a mangé une fuit' } & &
\end{array}
$$

$$
\begin{aligned}
& \text { João i-kokot- } \varnothing \text { Sete de Setembro dewota kyyn. } \\
& \text { João PART-traverser-ABS Sete de setembro autre.côté à } \\
& \text { 'João a traversé la rue Sete de Setembro' }
\end{aligned}
$$

Pour ce qui est du système aspectuel, en Karitiana le morphème de non-futur peut être utilisé à la fois pour exprimer un aspect imperfectif et perfectif. Ce fait affecte directement la possibilité d'appliquer certains tests, comme le paradoxe de l'imperfectif, qui se fonde sur les implications différentes d'une phrase à l'aspect imperfectif selon que le prédicat soit atélique ou télique. Les phrases (44) et (45) illustrent le test en français. Si un événement décrit par un prédicat atélique comme celui exemplifié en (44a) est interrompu, il y a une implication de (44a) à (44b). Mais si un événement télique comme (45a) est arrêté, on ne peut pas dire que (45b) est vrai. Autrement dit, l'implication de l'imperfectif au perfectif n'est pas maintenue.

(44) a. Jean était en train de courir.

b. Jean a couru.

(45) a. Jean était en train de traverser la rue.

b. Jean a traversé la rue. 
Le test du paradoxe de l'imperfectif est inapplicable en Karitiana parce que la même forme verbale (le non-futur) peut avoir une lecture perfective ou imperfective. Par conséquent, il n'est pas possible de formuler la deuxième phrase du test de façon distincte. Les phrases en (46) et (47) montrent la tentative. La phrase (47) peut être interprétée comme 'João a traversé la rue Sete de Setembro' ou comme 'João était en train de traverser la rue Sete de Setembro'. Cette deuxième possibilité détient l'implication et fait échouer le test.

$\begin{array}{clll}\text { João i-kokot- } & \text { ty-ka-t } & \text { Sete de Setembro dewota } & \text { kyyn. } \\ \text { João PART-traverser-ABS } & \text { IMP-MOV.SG-NFUT } & \text { Sete de Setembro } & \text { autre.côté à } \\ \text { Atykiri } & \varnothing \text {-pyr-a-nanda-gn } & \text { i. } & \\ \text { alors } & \text { 3-ASS-PASS-renverser-NFUT } & \text { il } & \end{array}$

'João était en train de traverser la rue Sete de Setembro. Alors il a été renversé'

\author{
João i-kokot- $\varnothing \quad$ Sete de Setembro dewota kyyn. \\ João PART-traverser-ABS Sete de setembro autre.côté à \\ 'João a traversé la rue Sete de Setembro' \\ 'João était en train de traverser la rue Sete de Setembro'
}

Un test qui a été appliqué avec succès est le test de l'ambiguïté avec presque, qui caractérise les accomplissements parce qu'il peut faire référence soit à la borne initiale, soit à la borne finale de l'événement. Les activités, qui n'ont pas de borne finale naturelle, ne donnent pas lieu à cette ambiguïté. Les phrases en (48) montrent le fonctionnement de ce test. La phrase (48a) a seulement une interprétation selon laquelle Jean allait courir, mais n'a pas commencé à courir. (48b) par contre peut être utilisée tant dans une situation où Jean allait dessiner un cercle, mais n'a pas commencé, que dans une situation dans laquelle Jean a commencé à dessiner un cercle et s'est arrêté avant la fin de l'action.
a. Jean a presque couru.
b. Jean a presque dessiné un cercle. 
Le test a été appliqué en Karitiana en utilisant le suffixe $\{-w a k\}$ qui signifie 'vouloir'3. La phrase (49) ne peut être utilisée que dans les contextes où João n'a pas commencé à courir. La phrase (50) est pour sa part ambiguë. Elle peut être utilisée dans un contexte où João a l'intention de commencer à peindre l'école ou dans un contexte dans lequel il a commencé à peindre et a l'intention de finir.

$$
\begin{aligned}
& \text { João Ø-na-aka-t } \quad \text { i-pykyn- }<\text { i }>\text {-wak. } \\
& \text { João 3-DECL-COP-NFUT PART-COurir-DES } \\
& \text { 'João veux courir' } \\
& \text { Situations: } \checkmark \quad \text { il veut commencer à courir } \\
& \text { × il a commencé à courir }
\end{aligned}
$$

$$
\begin{aligned}
& \text { João Ø-naka-pog-<i>-wak pyejepa goot. } \\
& \text { João 3-DECL-peindre.en.blanc-DES école nouveau } \\
& \text { 'João veux peindre en blanc la nouvelle école' } \\
& \text { Situations: } \checkmark \quad \text { il veut commencer à peindre } \\
& \checkmark \quad \text { il a commencé à peindre et il veut finir }
\end{aligned}
$$

\subsection{Cumulativité et Comptabtabilité}

Dans la deuxième partie de ce chapitre, nous argumentons que les prédicats verbaux événementiels sont cumulatifs et comptables en Karitiana, malgré le fait que ces deux propriétés semblent à première vue incompatibles. L'incompatibilité apparente surgit de l'association courante entre la propriété de cumulativité et les prédicats massifs. Notre argumentation se fonde sur l'analyse de la pluriactionnalité et de la quantification adverbiale dans cette langue.

En Karitiana les syntagmes verbaux sont cumulatifs dans le sens où ils ont le nombre neutre. Les phrases sans quantification adverbiale sont appropriées dans des contextes avec un ou plus d'un événement.

\footnotetext{
3 Avant de trouver le bon test, nous avons essayé bien d'autres possibilités, à savoir: (i) la compatibilité avec des modificateurs en/pour X temps; (ii) le paradoxe de l'imperfectif; (iii) la compatibilité avec un morphème de progressif; (iv) la compatibilité avec la continuation et Marie a fait la même chose; et (v) la compatibilité avec l'impératif.
} 
João i-'ot-Ø.

João PART-tomber-ABS

'João a tombé'

Situations: $\checkmark$ une fois

$\checkmark$ plus d'un fois

Par contre, quand il y a un morphème pluriactionnel la phrase entraîne nécessairement que plus d'un événement du type décrit a eu lieu.

João i-'ot-'ot-Ø.

João PART-tomber-DUPL-ABS

'João a tombé (plus d'un fois)'

Situations: $\quad x$ une fois

$\checkmark \quad$ plus d'un fois

L'un des arguments qui montrent que les syntagmes verbaux en Karitiana sont comptables est le fait que les morphèmes pluriactionnnels peuvent se combiner avec tous les types de prédicats verbaux (sauf les états permanents décrits par des prédicats individuallevel) et les phrases obtenues ont toujours une interprétation itérative. Les phrases (53) et (54) montrent que les prédicats téliques peuvent être pluralisés en Karitiana.
a. João i-kokot- $\varnothing$ Sete de Setembro dewota kyyn.
João PART-traverser-ABS Sete de Setembro autre.côté à
'João a traversé la rue Sete de Setembro'
b. João i-kokot-kokot- $\varnothing \quad$ Sete de Setembro dewota kyyn.
João PART-traverser-DUPL-ABS Sete de Setembro autre.côté à
'João a traversé la rue Sete de Setembro (plus d'une fois)'
a. João Ø-naka-typ-Ø Antônio Bigode.
João 3-DECL-trouver-NFUT Antônio Bigode
'João a trouvé Antonio Bigode' 


\section{b. João Ø-naka-typ-typ- $\varnothing \quad$ Antônio Bigode. \\ João 3-DECL-trouver-DUPL-NFUT Antônio Bigode \\ 'João a trouvé Antonio Bigode (plus d'une fois)'}

Les prédicats atéliques peuvent aussi être pluralisés avec une interprétation itérative. Cela indique qu'ils sont aussi comptables.
a. João i-pykyn-<a $>$-t.
João PART-courir-ABS
'João a couru'
b. João i-pykyn-pykyn-<a $>$-t.
João PART-Courir-DUPL-ABS
'João a couru (plus d'une fois)'
Situations: $\quad \checkmark \quad$ a couru plus d'une fois
$x$ a couru intensément une seule fois

(56)
a. Inácio Ø-na-aka-t i-osedn-Ø.
Inácio 3-DECL-COP-NFUT PART-être.heureuX-ABS
'Inácio était heureux'
b. Inácio Ø-na-aka-t i-osedn-osedn-Ø.
Inácio 3-DECL-COP-NFUT PART-être.heureuX-DUPL-ABS
'O Inácio était heureux (plus d'une fois)'
Situations: $\quad \checkmark$ était heureux plus d'une fois
$x$ était très heureux une seule fois

Le fait d'affirmer qu'un certain domaine a une dénotation en même temps cumulative et comptable peut sembler paradoxal, en raison du fait que la propriété de la cumulativité est traditionnellement attribuée à des prédicats massifs (Link 1983). Toutefois, la cumulativité n'est pas la caractéristique qui différencie les prédicats comptables des massifs. 
Dans la théorie de Link (1983), la cumulativité est une propriété des prédicats massifs et pluriels et n'est donc pas la caractéristique qui permet la distinction entre prédicats comptables et massifs. Dans la proposition de l'auteur, la distinction massif-comptable est basée sur la propriété d'homogénéité. À première vue, la cumulativité et l'homogénéité semblent tout à fait similaires. Mais pour décrire le comportement des prédicats dans notre recherche, leur différence est cruciale. Les définitions (57) et (58) (basées sur Krifka 1992 et Rothstein 2010) montrent que l'homogénéité est une propriété de prédicats qui est attribué à des parties, c'est une propriété descendante. D'autre part la cumulativité est une propriété ascendante, qui caractérise le prédicat en fonction de la somme de ses parties.

Homogénéité:

Un prédicat $\mathrm{P}$ est homogène si: pour tout $\mathrm{x} \in \mathrm{P}$, et pour tout $\mathrm{y}$ : $\mathrm{y} \leq \mathrm{x} \wedge \neg \mathrm{y}=\mathrm{x}$

$\rightarrow \mathrm{y} \in \mathrm{P}$

"Un prédicat $\mathrm{P}$ est homogène si, et seulement si, pour tout $\mathrm{x}$ tel que $\mathrm{x}$ appartient au prédicat $\mathrm{P}$, toutes les parties $\mathrm{y}$ de $\mathrm{x}$, qui sont différents de $\mathrm{x}$, appartiennent aussi au prédicat P"

(58) Cumulativité:

Un prédicat $\mathrm{P}$ est cumulatif si: $\mathrm{x} \in \mathrm{P} \wedge \mathrm{y} \in \mathrm{P} \wedge \neg \mathrm{x}=\mathrm{y} \rightarrow \mathrm{x} \square \mathrm{y} \in \mathrm{P}$

"Un prédicat $\mathrm{P}$ est cumulatif si, et seulement si, lorsqu'il existe un $\mathrm{x}$ et un y qui appartiennent au prédicat $\mathrm{P}$, et que $\mathrm{x}$ et $\mathrm{y}$ sont différents entre eux, la somme de $\mathrm{x}$ et $\mathrm{y}$ appartient aussi au prédicat $\mathrm{P}$ ”

Alors la conclusion générale du chapitre 3 est qu'il existe une différence entre prédicats téliques et atéliques en Karitiana et que les prédicats verbaux événementiels sont cumulatifs et comptables dans cette langue. 


\section{6 \\ La Modification de Degré en Karitiana}

\subsection{Introduction}

Le quatrième chapitre se concentre sur la modification de degré en Karitiana telle qu'elle est exprimée par l'adverbe pita(t) 'vrai/très/beaucoup'. Nous avons constaté que pita(t) peut apparaître dans le domaine nominal, adjectival et verbal. Son sens dépend du type d'échelle associée au prédicat modifié.

Les modificateurs de degré ont certaines caractéristiques qui les distinguent des autres adverbes. Par exemple, ils ont une certaine liberté par rapport au type de syntagme qu'ils modifient qui n'est pas partagée par d'autres types d'adverbe. L'adverbe beaucoup, p. ex., peut modifier des substantifs comptables et massifs (59a et 59b) et des verbes (60):
a. beaucoup de livres
b. beaucoup de soupe

(exemples 16a et 16b de Doetjes 2007,p. 11)

(60) Sylvie va beaucoup au cinéma.

(exemple 1a de Doetjes 2007, p. 1)

La modification de degré est en général comprise comme une opération sur des prédicats gradables. Kennedy et McNally (2005) proposent une typologie sémantique des prédicats gradables selon deux propriétés principales: (i) la division entre relatif et absolu par rapport à un paramètre de comparaison; (ii) l'association avec une structure scalaire ouverte ou fermée. 


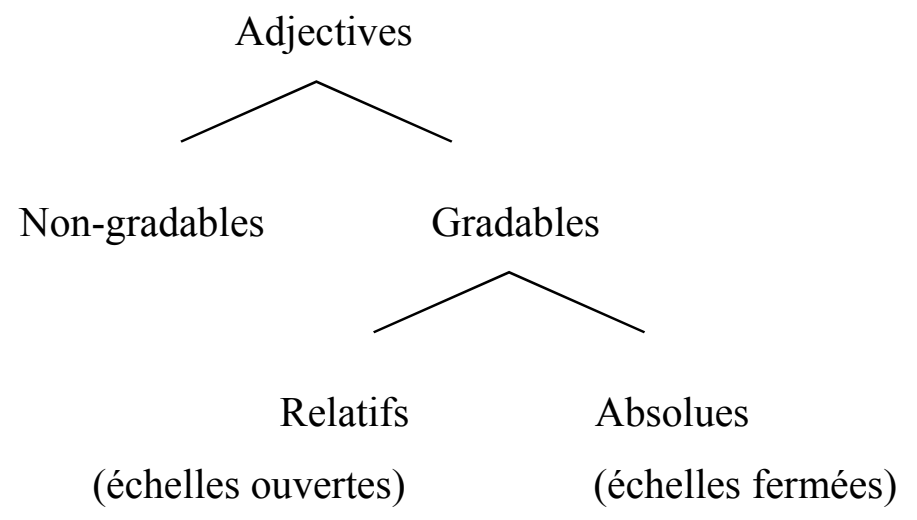

Les prédicats relatifs sont ceux qui dépendent d'un paramètre de comparaison (standard of comparison en anglais), alors que les prédicats absolus n'ont pas de paramètre de comparaison dépendant du contexte. Par exemple, un adjectif comme haut est un prédicat relatif, car sa dénotation varie contextuellement. Pour attribuer à une entité la propriété d'être haut, il est nécessaire de prendre en compte le contexte. Les prédicats relatifs donnent lieu à des phrases dont les conditions de vérité varient en fonction d'une classe de comparaison. L'adjectif plein, par contre, n'a pas son paramètre de comparaison défini contextuellement. Il n'est pas nécessaire de comparer avec d'autres objets qui soient pleins dans le contexte pour affirmer que quelque chose est pleine.

Dans le modèle de la sémantique scalaire, les échelles sont des constructions formelles définies comme un ensemble de degrés ordonnés sur une dimension. Selon Kennedy et McNally (2005), les échelles associées aux prédicats gradables se divisent en deux types: ouvertes et fermées. Les échelles ouvertes sont celles qui n'ont pas un degré minimum ou maximum déterminé lexicalement. Haut, p. ex., est un adjectif d'échelle ouverte, puisque il n'y a pas un degré minimum ou maximum pour évaluer la hauteur d'un individu. En revanche, les échelles fermées possèdent un degré minimum et maximum. Par exemple, plein et vide sont des adjectifs d'échelle fermée. L'échelle d'occupation associée à ces adjectifs possède un degré minimum, associé à vide, et un degré maximum, associé à plein.

Seuls les adjectifs d'échelle fermée peuvent apparaitre dans des phrases avec des modificateurs de proportion tels que l'adverbe complètement :

(61) a. ? Luciana est complètement haute.

b. Le verre est complètement plein. 
La structure de l'échelle a une influence sur le choix du paramètre de comparaison contextuelle. Les adjectifs associés à des échelles ouvertes sont liés à des paramètres de comparaison relatifs, alors que les adjectifs d'échelle fermée utilisent des paramètres de comparaison absolus pour être évalués.

La sémantique scalaire formalise les prédicats gradables en ajoutant un type sémantique $\mathbf{d}$ de degré au domaine. Les adjectifs gradables dénotent une relation entre degrés et individus:

$$
\llbracket \text { cher } \rrbracket=\lambda d \lambda x \text {. "coût" }(x)=d
$$

La dénotation (62) signifie que cher est un prédicat qui prend un argument de type d et un argument de type $\mathbf{x}$ et retourne l'appariement de l'argument $\mathbf{x}$ à un degré $\mathbf{d}$ sur une échelle de coût. L'adjectif a le type $<\mathrm{d},<\mathrm{e}, \mathrm{t}>>$. Dans la dénotation (62), "coût" est une fonction de mesure qui projette ses arguments sur des représentations abstraites (les degrés des échelles). Ainsi, une généralisation peut être faite à propos de la dénotation des adjectifs gradables. Dans la dénotation (63), $\mathbf{g}_{\text {adj }}$ est la fonction de mesure associée à l'adjectif.

$$
\llbracket \operatorname{Adj}_{\mathrm{g}} \rrbracket=\lambda \mathrm{d} \lambda \mathrm{x} \cdot \mathrm{g}_{\mathrm{adj}}(\mathrm{x})=\mathrm{d}
$$

Les prédicats gradables de type $<\mathrm{d},<\mathrm{e}, \mathrm{t}>>$ deviennent des prédicats de type $<\mathrm{e}, \mathrm{t}>$ grâce à la morphologie de degré. Les cas où il n'y a pas de morphologie de degré explicite sont considérés comme la forme positive de l'adjectif. Dans ces cas, la théorie postule qu'il y a un morphème nul de degré pos qui lie la variable représentée par $\lambda \mathrm{d}$.

$$
\llbracket p o s \rrbracket=\lambda \mathrm{G}_{<\mathrm{d},<e, \triangleright>} \lambda \mathrm{x}_{\mathrm{e}} . \exists \mathrm{d}\left[\mathrm{G}(\mathrm{d})(\mathrm{x}) \& \mathrm{~d} \geq \mathrm{d}_{\mathrm{s}}\right]
$$

Dans la dénotation (64), $\mathbf{G}$ est une variable pour les adjectifs gradables de type $<\mathrm{d}<\mathrm{e}, \mathrm{t}>>$, $\mathbf{x}$ et $\mathbf{d}$ sont les variables des individus et des degrés (de type e et $\mathbf{d}$ respectivement) et $\mathbf{d}_{\mathbf{s}}$ est le degré du paramètre de comparaison approprié. Selon Kennedy (2007) le degré $\mathbf{d}_{\mathbf{s}}$ est calculé en utilisant à la fois les propriétés de l'adjectif et du contexte d'énonciation.

Le degré $\mathbf{d}_{\mathbf{s}}$ est le degré normal des adjectifs relatifs. Il est le degré qui dans un contexte donné constitue le seuil (cutoff point) qui sépare les individus qui ont la propriété 
dénotée par l'adjectif de ceux qui ne l'ont pas. Quand l'adjectif est associé a une échelle fermée, le degré $\mathbf{d}_{\mathbf{s}}$ n'est pas le degré normal mais plutôt le degré maximal de l'échelle. Mais il est également considéré le degré seuil de l'échelle. La notion de degré seuil sera centrale dans l'analyse que nous proposons pour la sémantique de l'adverbe pita(t).

\subsection{Distribution de pita(t)}

La distribution de pita(t) montre que le suffixe $\{-t\}$ est exclu de l'utilisation $d u$ modificateur avec des prédicats non verbaux. C'est à cause de cette distribution qu'il a été glosé comme adverbialisateur dans cette thèse. Beaucoup d'autres adverbes en Karitiana présentent le même morphème. L'interprétation de pita(t) varie en fonction du type de prédicat. Les sections suivantes montrent la distribution et l'interprétation de pita(t) avec des syntagmes adjectivaux, verbaux et nominaux.

\subsubsection{Syntagmes Adjectivaux}

Quand pita(t) modifie des syntagmes adjectivaux, il signifie 'très' si l'adjectif est associée à une échelle ouverte, comme $s e^{\prime} a$ 'bon', et 'complètement' si l'adjectif est associée à une échelle fermée, comme akydop 'ouvert'.

$$
\begin{aligned}
& \text { Õwã se'a pita i-otam- } \varnothing . \\
& \text { garçon bon très PART-arriver-ABS } \\
& \text { 'Le très bon garçon est arrivé' }
\end{aligned}
$$

$$
\begin{array}{llll}
\text { Karamã } & \text { akydop pita } & \text { i-pot- } \varnothing . \\
\text { porte } & \text { ouvert } & \text { complètement } & \text { PART-casser-ABS }
\end{array}
$$

'La porte complètement ouverte a cassé'

Cela signifie que lorsque pita modifie les adjectifs d'échelle ouverte, il détermine un degré supérieur au degré normal associée à l'échelle de l'adjectif. Par contre, quand il s'applique à des adjectifs d'échelle fermée, il sélectionne le degré maximal de l'échelle. Cette distribution de pita indique qu'il y a une ressemblance entre le degré normal des adjectifs 
associés à des échelles ouvertes et le degré maximal des adjectifs de échelles fermées. Nous avons vu que ce degré peut être conçu comme le degré seuil de l'échelle.

Pour analyser pita(t), nous adoptons dans cette thèse la proposition de Kennedy (2007) pour le morphème pos, dont le paramètre et la classe de comparaison sont traduits sous la forme du degré $\mathbf{d}_{\mathbf{s}}$ qui est déterminé en fonction des propriétés des adjectifs et du contexte d'énonciation. On propose que pita a la même dénotation que le morphème pos en raison des différences des paramètres contextuels.

Le comportement de pita avec des adjectifs d'échelle fermée est un argument en faveur de ce choix. Comme indiqué ci-dessus, lorsque pita est utilisé pour modifier les adjectifs d'échelle fermée, il sélectionne le degré maximal de l'échelle désignée par l'adjectif. C'est exactement le même sens du morphème pos. La dénotation proposée est la suivante:

$$
\llbracket \text { pita } \rrbracket=\lambda \mathrm{G}_{<\mathrm{d},<\mathrm{e}, \triangleright>} \lambda \mathrm{x}_{\mathrm{e}} \cdot \exists \mathrm{d}\left[\mathrm{G}(\mathrm{d})(\mathrm{x}) \& \mathrm{~d} \geq \mathrm{d}_{\mathrm{s}}\right]
$$

Tout comme dans le cas de la dénotation du morphème pos proposée par Kennedy (2007), si l'adjectif a une échelle ouverte, le degré $\mathbf{d}_{\mathbf{s}}$ est représenté par le degré normal, et la sémantique de l'expression est que le degré d est supérieur au degré normal. Si l'adjectif est d'échelle fermée, $\mathbf{d}_{\mathbf{s}}$ est représenté par le degré maximal de l'échelle, et le sens de la phrase est que le degré d est égal au degré maximal de l'échelle.

Nous avons constaté que le modificateur de degré pita ne sélectionne pas un type de prédicat particulier, contrairement à ce qui se passe avec les modificateurs de degré very, much et well en anglais (Kennedy et McNally 2005). Cependant, l'interprétation de pita varie selon le prédicat modifié. Si le prédicat a une d'échelle ouverte, sa signification est quelque chose comme "supérieur au degré normal de l'échelle", le sens habituellement attribué au modificateur très. Si le prédicat est d'échelle fermée, son interprétation est "égal au degré maximal de l'échelle", ce qui peut être rendu par complètement.

Malgré cette variation, il est possible de fournir une analyse unifiée pour les phrases contenant des adjectifs modifiés par pita. Nous avons proposé une entrée lexicale unique pour le modificateur sur la base du degré $\mathbf{d}_{\text {s }}$ qui correspond au point de coupure ou seuil de l'échelle. 


\subsubsection{Syntagmes Verbaux}

Dans le domaine verbal, la distribution de $\operatorname{pita}(t)$ est restreinte: ce n'est que dans des phrases avec des prédicats atéliques qu'il peut avoir une interprétation de degré. Les exemples (68) et (69) illustrent le cas des prédicats verbaux d'activité et d'état respectivement.

$$
\begin{array}{lll}
\text { João } & \text { i-pykyn- }<\mathrm{a}>-\mathrm{t} & \text { pita-t. } \\
\text { João } & \text { PART-courir-ABS } & \text { beaucoup-ADV }
\end{array}
$$

'João a couru beaucoup'

$$
\begin{array}{lll}
\text { João } Ø \text {-na-aka-t } \quad \text { i-osedn- } \varnothing & \text { pita-t. } \\
\text { João 3-DECL-COP-NFUt } & \text { PART-être.heureux-ABS } & \text { beaucoup-ADV } \\
\text { 'João a été très heureux' } &
\end{array}
$$

Une caractéristique surprenante de la modification de degré est que les phrases (68) et (69) avec pita(t) peuvent être utilisées dans plus d'une situation, indiquant le haut degré dans plusieurs dimensions différentes (la durée, le nombre d'itérations, l'intensité, etc.), comme on peut le constater en (70) et (71).

(70) Situations dans lesquelles la phrase (68) peut être utilisée:
a. João a couru pendant longtemps;
b. João a couru avec une grande vitesse;
c. João a couru une grande distance;
d. João a couru plusieurs fois;
e. João a couru très intensément, en faisant beaucoup d'effort.

(71) Situations dans lesquelles la phrase (69) peut être utilisée:
a. João a été heureux pendant longtemps;
b. João a été heureux plusieurs fois;
c. João a été très heureux. 
Ceci contraste avec l'interprétation unique qu'on obtient dans le cas des prédicats téliques. Comme le montrent les phrases (72) et (73), quand on utilise pita(t) avec des accomplissements et des achèvements, il ne peut se traduire que comme 'en effet', 'vraiment'.

$$
\begin{array}{lll}
\text { João i-tat- } \varnothing & \text { pita-t } & \text { Porto Velho pip. } \\
\text { João PART-aller-ABS } & \text { beaucoup-ADV } & \text { Porto Velho à }
\end{array}
$$

'En effet, João est allé à Porto Velho'

$$
\begin{array}{lll}
\text { João } & \text { i-otam- } \varnothing & \text { pita-t. } \\
\text { João } & \text { PART-arriver-ABS } & \text { beaucoup-ADV }
\end{array}
$$

'En effet, João est arrivé'

Il faut donc développer une analyse de pitat qui explique cette interprétation de confimation avec les prédicats téliques, qui diffère de l'interprétation associé aux échelles indéterminées avec les prédicats atéliques. Les prédicats téliques, puisqu'ils ont une fin déterminée (le telos), peuvent être considérés comme des prédicats d'échelle fermée. Les prédicats atéliques, par contre sont considérés comme des prédicats d'échelle ouverte (Caudal e Nicolas 2005).

Pourtant, à la différence des adjectives gradables, les prédicats verbaux ne sont pas associés à des échelles dans le lexique. Dans l'analyse que nous proposons, une fonction de degré Deg, qui prend le prédicat verbal comme argument, est responsable pour l'introduction d'un argument de degré et d'une variable d'échelle dans la dénotation de ce type de verbes au cours de la dérivation sémantique. Deg est une fonction qui s'applique à de prédicats de type $<$ s, $\mathrm{t}>$ et retourne une relation entre des degrés et des événements de type $<$ d, $<$ s, $\mathrm{t}>>$. Afin de capturer l'indétermination des échelles, une fonction de mesure $\boldsymbol{\mu}$ est utilisée comme une variable de dimensions (Krifka 1998, Nakanishi 2007, Thomas 2009).

$$
[[\text { Deg }]]=\lambda \mathrm{P}_{<e,<s, \triangleright>>} \lambda d \lambda x \lambda e . P(x)(e) \& \mu(e)=d
$$

Comparons, par exemple, la dénotation du verbe pykyn 'courir' avant et après se combiner avec la fonction de degré: 


$$
\begin{array}{ll}
\text { a. } & {[[\text { pykyn }]]=\lambda \mathrm{x} \lambda \mathrm{e} \cdot \operatorname{courir}(\mathrm{x})(\mathrm{e})} \\
\text { b. } & {\left[\left[\text { pykyn }_{\mathrm{deg}}\right]\right]=\lambda \mathrm{d} \cdot \lambda \mathrm{x} \lambda \mathrm{e} \cdot \operatorname{courir}(\mathrm{x})(\mathrm{e}) \& \mu(\mathrm{e})=\mathrm{d}}
\end{array}
$$

Nous soutenons que les échelles associées à des verbes atéliques ne sont pas disponibles dans le lexique, mais elles sont indéterminées, ce qui est représenté par la variable $\boldsymbol{\mu}$. Cette variable pourra être remplie, par exemple, par des dimensions comme la durée, la vitesse, la distance, etc. Cela signifie que quand pitat modifie des prédicats atéliques, il opère sur des échelles fournies contextuellement. Son interprétation est celle d'un degré supérieur au degré normal des échelles qui peuvent être associées au prédicat.

Comme mentionné précédemment, les prédicats téliques peuvent être considérés comme des prédicats d'échelle fermée. Lorsque pita est utilisé pour modifier les adjectifs d'échelle fermé, il sélectionne le degré maximal de l'échelle associée à l'adjectif, ayant une interprétation de complètement. C'est le même processus que l'on trouve dans les prédicats téliques modifiés par pitat. L'adverbe sélectionne également le degré maximal. Le degré maximal des échelles lexicalement associées à ces prédicats est le telos de l'événement. Cela signifie que lorsque pitat est utilisé avec un prédicat télique, il indique que le telos de l'événement a été atteint. D'où sa traduction plus habituelle pour 'en effet', comme confirmation de l'existence d'un événement du type décrit par le verbe télique.

La façon dont la variable des échelles $\mu$ est remplie permet de dériver la différence entre la modification des prédicats téliques et atéliques. Notre analyse repose sur l'hypothèse que l'indétermination est une caractéristique propre aux prédicats atéliques. Les prédicats verbaux téliques, par contre, obtiennent aussi un argument de degré et une variable d'échelle dans la combinaison avec la fonction Deg, mais la variable d'échelle est fixée: il s'agit de l'échelle associée univoquement au type d'événement dénoté par le verbe.

$$
\begin{array}{ll}
\text { a. } & {[[\text { arriver }]]=\lambda \mathrm{x} \lambda \mathrm{e} \text {. } \operatorname{arriver}(\mathrm{x})(\mathrm{e})} \\
\text { b. } & {\left[\left[\text { arriver }_{\mathrm{deg}}\right]\right]=\lambda \mathrm{d} . \lambda \mathrm{x} \lambda \mathrm{e} \cdot \operatorname{arriver}(\mathrm{x})(\mathrm{e}) \& \text { “arriver” }(\mathrm{e})=\mathrm{d}}
\end{array}
$$

Finalement, on propose la dénotation suivante pour pitat :

$$
[[\text { pitat }]]=\lambda \mathrm{G}_{<\mathrm{d},<e,<, \text { s, } \gg>>} \lambda \mathrm{x}_{<\mathrm{e}>} \lambda \mathrm{e}_{<\mathrm{s}\rangle} . \exists \mathrm{d}\left[\mathrm{G}(\mathrm{d})(\mathrm{x})(\mathrm{e}) \& \mathrm{~d} \geq \mathrm{d}_{\mathrm{s}}\right]
$$


Pitat est un fonction qui s'applique à un argument $\mathbf{G}$ de type $<\mathrm{d},<\mathrm{e}<\mathrm{s}, \mathrm{t}>>>$ (le verbe gradable), un argument individuel $\mathbf{x}$ de type e et un argument événementiel $\mathbf{e}$ de type $\mathbf{s}$, et retourne une phrase dont la fonction gradable est appliquée à $\mathbf{d}, \mathbf{x}$, et $\mathbf{e}$ et affirme qu'il existe un degré $\mathbf{d}$ qui est supérieure ou égale à $\mathbf{d}_{\mathbf{s}}$

Comme dans le cas des adjectifs, dans la formule (77), ds représente le degré pertinent pour chaque type de prédicat. Si le prédicat a une échelle ouverte (prédicat atélique) $\mathbf{d}_{\mathrm{s}}$ représente le degré normal de l'échelle, si le prédicat a une échelle fermée (prédicat télique), $\mathbf{d}_{\mathrm{s}}$ représente le degré maximal de l'échelle.

\subsubsection{Syntagmes Nominaux}

En plus de modifier des prédicats adjectivaux et verbaux, pita(t) peut être utilisée comme modificateur des substantifs. Dans ces cas il est interprété comme 'vrai' ou 'véritable'.

$$
\begin{array}{ll}
{\left[\begin{array}{ll}
\text { Taso pita } & \text { i-otam- } \varnothing . \\
\text { homme vrai } & \text { PART-arriver-ABS }
\end{array}\right.}
\end{array}
$$

'Le vrai homme est arrivé'

Pour analyses ces phrases on adopte la proposition de Morzycki (2011) selon laquelle les substantifs peuvent être associés à une échelle de précision. Et lorsque le degré de précision est maximal, le nom doit être interprété de façon précise.

$$
[[\text { homme }]]=\lambda \mathrm{d} \lambda \mathrm{x} \text {. "précision" }(\mathrm{x})=\mathrm{d}
$$

Nous proposons que les substantifs obtiennent cette échelle par une fonction $\mathbf{D e g}_{\text {subst }}$ inspiré par la fonction Deg précédemment utilisé.

$$
\left[\left[\operatorname{Deg}_{\text {subst }}\right]\right]=\lambda \mathrm{P}_{<\mathrm{e}, \triangleright} . \lambda \mathrm{d} \lambda \mathrm{x} . \mathrm{P}(\mathrm{x}) \& \text { "précision" }(\mathrm{x})=\mathrm{d}
$$

Notre analyse entraîne que la même version de pita qui modifie des adjectifs modifie aussi des substantifs dans leur version gradable avec une échelle de précision. Comme l'échelle de précision est une échelle fermée, pita sélectionne le degré maximal de précision. 
Dans ce cas, son sens est que le nom modifié doit être interprété de la manière la plus précise possible, la plus proche de l'idéal. D'où son interprétation de 'véritable'.

\subsection{Proposition Unifiée}

Malgré la multiplicité d'interprétations contextuelles, pita(t) peut être analysée uniformément comme un modificateur de degré dans tous les domaines. Cela implique qu'il exige toujours de se combiner avec un prédicat qui possède un argument de type d. Cependant, notre analyse a présenté deux versions de pita(t), un modificateur de noms et d' adjectifs et un modificateur des prédicats verbaux. Selon cette analyse pita, la forme qui se combine avec des noms et des adjectifs, est un modificateur de degré de type $<<\mathrm{d},<\mathrm{e}, \mathrm{t}>>,<\mathrm{e}, \mathrm{t}>>$ et pitat, la forme qui apparaît comme un adverbe dans le prédicat de la phrase, est de type $<<\mathrm{d},<\mathrm{e},<\mathrm{s}, \mathrm{t}>>>$, $<\mathrm{e},<\mathrm{s}, \mathrm{t}>>>$.

$$
\begin{array}{ll}
\text { a. } & {[[\text { pita }]]=\lambda \mathrm{G}_{<\mathrm{d},<\mathrm{e}, \gg>} \lambda \mathrm{x}_{<\mathrm{e}>} . \exists \mathrm{d}\left[\mathrm{G}(\mathrm{d})(\mathrm{x}) \& \mathrm{~d} \geq \mathrm{d}_{\mathrm{s}}\right.} \\
\text { b. }[[\text { pitat }]]=\lambda \mathrm{G}_{<\mathrm{d},<\mathrm{e},<\mathrm{s}, \downarrow>>} \lambda \mathrm{x}_{<\mathrm{e}>} \lambda \mathrm{e}_{<\mathrm{s}>} . \exists \mathrm{d}\left[\mathrm{G}(\mathrm{d})(\mathrm{x})(\mathrm{e}) \& \mathrm{~d} \geq \mathrm{d}_{\mathrm{s}}\right]
\end{array}
$$

Les dénotations ci-dessus montrent que le sens de pita(t) est essentiellement le même dans tous les domaines. La seule différence est la présence d'un type $\mathbf{s}$ des événements dans la dénotation de pitat. Le suffixe $\{-t\}$ est responsable pour fournir l'argument de type $\mathbf{s}$ des événements et s'applique à pita pour engendrer pitat:

$$
[[-t]]=\lambda \mathrm{M}_{<<\mathrm{d},<\mathrm{e}, \downarrow>,<\mathrm{e}, \downarrow>>} \lambda \mathrm{G}_{<\mathrm{d},<\mathrm{e},<\mathrm{s}, \downarrow>>>} \lambda \mathrm{x}_{1} \lambda \mathrm{e}_{1} . \mathrm{M}\left(\lambda \mathrm{d}_{1} \lambda \mathrm{x}_{2} . \mathrm{G}_{<\mathrm{d},<\mathrm{e},<\mathrm{s}, \downarrow>>}\left(\mathrm{d}_{1}\right)\left(\mathrm{x}_{2}\right)\left(\mathrm{e}_{1}\right)\right)\left(\mathrm{x}_{1}\right)
$$

\section{$7 \quad$ Conséquences de la Proposition}

L'analyse sémantique de pita(t) défendue dans cette thèse a un certain nombre de conséquences. La première conséquence concerne la différence entre l'interprétation de degré et l'interprétation itérative, tel qu'elle est introduite dans le travail de Doetjes (2007). Grâce à l'indétermination des échelles associées aux verbes atéliques que nous postulons, il est possible d'expliquer l'interprétation itérative comme étant une variante de la modification de degré. 
Par conséquent, contrairement à la proposition de Doetjes (2007), l'itérativité est considérée un sous-type de la modification de degré, et non pas une opération qui est en concurrence avec elle. En fait, dans la perspective adoptée dans cette thèse, il n'y a aucune interprétation génuinement itérative pour pitat. Il s'agit d'une modification de degré (ou une opération de degré) et l'interprétation itérative est engendrée par cette opération au même titre que celles d'intensité ou de durée.

Une autre conséquence de cette recherche est l'importance de l'indétermination pour la typologie des prédicats gradables. Nous avons proposé une variable d'échelles $\boldsymbol{\mu}$ pour analyser la modification des syntagmes verbaux par pita $(t)$. Cela indique que l'indétermination est aussi importante pour la typologie des prédicats gradables que l'ouverture des échelles associés au prédicat et son rapport au paramètre de comparaison. C'est précisément l' une des contributions théoriques de ce travail. En plus des paramètres connus basés sur la distinction entre échelle fermée et ouverte et entre prédicats relatifs et absolus, la propriété d'être ou non déterminée lexicalement est pertinente pour le fonctionnement des échelles.

En fait, étant donné qu' il y a une correspondance biunivoque entre les deux critères proposés par Kennedy et McNally (2005), il n'y a aucune raison de les considérer comme deux paramètres différents. Notre proposition est qu'ils peuvent être traités comme un seul paramètre décrit de deux manières différentes. La distinction relatif-absolu est une caractéristique des prédicats gradables et la distinction ouverte-fermée est une propriété des échelles associées aux prédicats gradables. Mais l'indétermination, quant à elle, constitue un paramètre indépendant, car elle permet la combinaison croisée avec les autres. Le tableau cidessous résume notre proposition pour la typologie sémantique des prédicats gradables et présente des exemples.

Table: Proposition pour la Typologie des prédicats gradables

\begin{tabular}{|c|c|c|c|}
\hline & $\begin{array}{c}\text { (In)détermination } \\
\text { d'échelles }\end{array}$ & \multicolumn{2}{|c|}{ Exemple } \\
\cline { 2 - 4 } & Adjectif & Verbe \\
\hline \multirow{2}{*}{$\begin{array}{c}\text { Prédicat Relatif } \\
\text { Échelle Ouverte }\end{array}$} & Déterminée & haut & $\begin{array}{c}\text { refroidir } \\
\text { (interprétation atélique) }\end{array}$ \\
\cline { 2 - 4 } & Indéterminée & grand & courir \\
\hline $\begin{array}{c}\text { Prédicat Absolu } \\
\text { Échelle Fermée }\end{array}$ & Déterminée & ouvert & arriver \\
\hline
\end{tabular}


Le tableau montre que les prédicats absolus ont toujours des échelles fermés et déterminées, alors que les prédicats relatifs ont des échelles ouvertes, mais celles-ci peuvent être divisés en deux types: déterminée et indéterminée. 


\section{ÍNDICE}

Forma de Apresentação dos Dados................................................................. 1

Abreviaturas das Glosas............................................................................. 2

Outras Abreviaturas.................................................................................................. 4

Outras Convenções....................................................................................................................... 4

Capítulo 1

Pano de Fundo

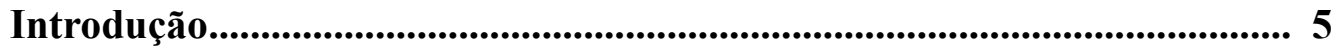

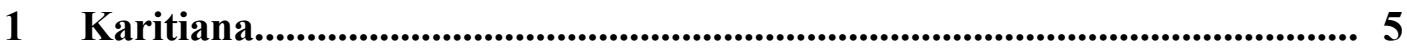

1.1 Ordem de Palavras e Padrão de Concordância.................................. 7

1.2 Modo, Tempo e Aspecto......................................................................... 14

1.2.1 Modo........................................................................... 14

1.2.2 Tempo..................................................................................... 19

1.2.3 Aspecto.................................................................. 21

1.3 Construção de Cópula....................................................................... 26

1.4 Sintagmas Nominais...................................................................... 30

2 Semântica Formal.......................................................................................... 34

2.1 Semântica de Eventos...................................................................... 40

Capítulo 2

Metodologia

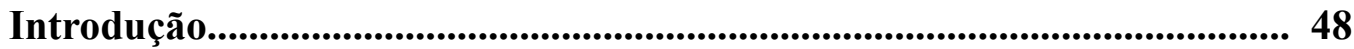

1 Metodologia da Pesquisa de Campo............................................................ 49

1.1 Teoria e Prática no Trabalho de Campo........................................... 50

1.2 Semântica Formal e Elicitação Controlada........................................ 51

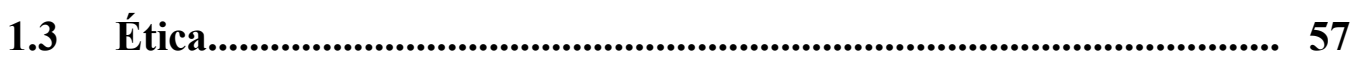

1.4 Considerações Finais.................................................................................... 60

2 A Divisão de Trabalho entre a Semântica e a Pragmática: Consequências Metodológicas..................................................................... 61

2.1 Semântica e Pragmática..................................................................... 61

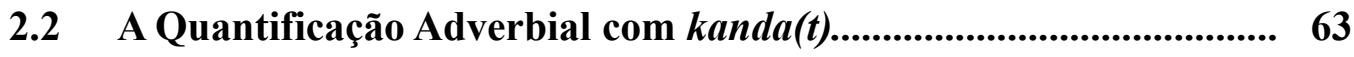

2.3 Proposta...................................................................................................64 64

2.3.1 Comparação com Propostas Semelhantes............................. 66

2.4 Caminhos da Proposta........................................................................... 68

2.4.1 Metodologia.................................................................. 68 


\section{Capítulo 3}

Sintagmas Verbais

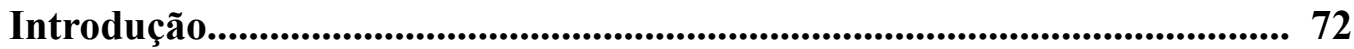

1 A Distinção Télico-Atélico e as Classes Acionais em Karitiana................... 73

1.1 Introdução....................................................................................... 73

$1.2 \quad$ Teorias sobre Acionalidade.................................................................. 75

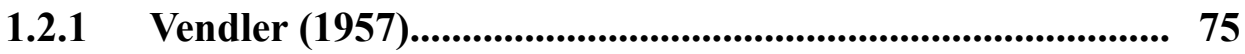

1.2.2 A Acionalidade como uma Propriedade de VPs.................... 79

1.2.3 Rothstein $(2004,2008)$............................................................ 84

1.2.4 Estativos stage-level e individual level.................................. 86

1.3 Classes Verbais em Karitiana............................................................... 89

1.3.1 Os Sintagmas Nominais e as Classes Acionais em Karitiana........................................................................... 89

1.3.2 Tabela de Verbos....................................................................... 94

1.3.3 Testes de Classes Acionais em Karitiana............................. 97

1.4 Considerações Finais.......................................................................... 129

2 Cumulatividade e Contabilidade...................................................................... 131

$2.1 \quad$ Dados do Karitiana......................................................................... 131

2.1.1 Cumulatividade........................................................... 131

2.1.2 _ Contabilidade.................................................................. 137

2.1.2.1 Advérbios de Frequência e de Grau em Francês. 137

2.1.2.2 Advérbios de Frequência e de Grau em Karitiana 139

2.1.2.3 Pluracionalidade.......................................................... 147

2.2 Discussão Teórica.............................................................................. 151

2.3 Cosiderações Finais.................................................................................... 154

Capítulo 4

A Modificação de Grau

Introdução................................................................................................................ 155

1 Grau: Uma Noção Especial......................................................................... 155

2 Semântica Escalar........................................................................................ 158

2.1 Predicados Relativos versus Predicados Absolutos............................. 160

2.2 Estrutura Escalar.......................................................................... 163

2.3 Relevância do Parâmetro de Comparação e da Estrutura Escalar. 
2.4 Formalização............................................................................. 170

3 Modificação de Grau em Karitiana....................................................... 175

3.1 Pita versus Pitat.............................................................................. 176

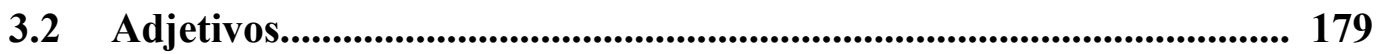

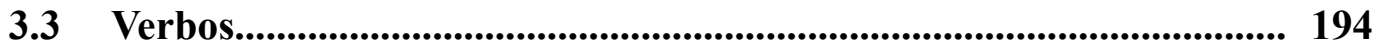

3.3.1 Graus e Escalas Associados aos Eventos 217

3.4 Substantivos......................................................................................... 231

4 Proposta de Formalização Unificada............................................................ 240

5 Consequências da Proposta............................................................................. 242

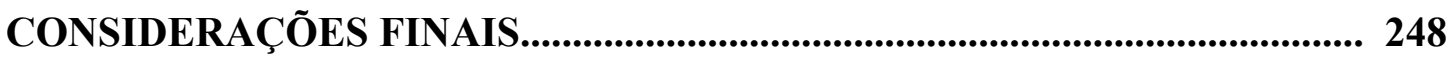

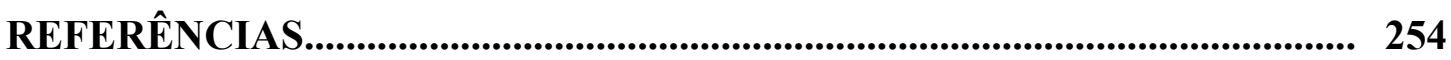




\section{ÍNDICE DE TABELAS}

Tabela 1 Prefixos de Concordância de Pessoa em Karitiana................................. 13

Tabela 2 Morfemas de Modo em Karitiana.......................................................... 15

Tabela 3 Sufixos de Tempo do Karitiana no Modo Declarativo............................. 20

Tabela 4 Morfemas de Imperfectivo em Karitiana................................................. 22

Tabela 5 Generalizações sobre a Construção de Cópula..................................... 28

Tabela 6 Tabela de Verdade da Conjunção............................................................... 43

Tabela 7 Classes Vendlerianas e sua Interação com o Tempo.............................. 78

Tabela 8 Proposta de Verkuyl (1996) Baseada nos Traços [ \pm ADD TO] e

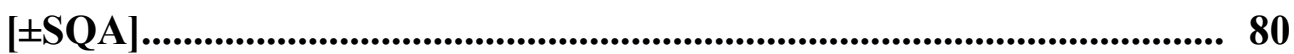

Tabela 9 Características de Rothstein (2004, 2008) para as Classes Vendlerianas.............................................................................................8 86

Tabela 10 Predicados de Atividade em Karitiana..................................................... 94

Tabela 11 Predicados Estativos Stage-level em Karitiana...................................... 95

Tabela 12 Predicados Estativos Individual-level em Karitiana................................. 95

Tabela 13 Predicados de Accomplishment em Karitiana......................................... 96

Tabela 14 Predicados de Achievement em Karitiana................................................... 96

Tabela 15 Teste em/por X tempo......................................................................... 98

Tabela 16 Teste em/por X tempo em Karitiana (Atividades e Accomplishments)... 99

Tabela 17 Teste em/por X tempo em Karitiana (Estativos)......................................... 102

Tabela 18 Teste do Paradoxo do Imperfectivo............................................................ 104

Tabela 19 Teste do Paradoxo do Imperfectivo em Karitiana..................................... 105

Tabela 20 Interrupção de Predicados de Accomplishment em Karitiana.............. 108

Tabela 21 Interrupção de Predicados de Atividade em Karitiana.......................... 109

Tabela 22 Interrupção de Predicados de Accomplishment em Karitiana com Descrição Complementar........................................................................... 110

Tabela 23 Interrupção de Predicados de Accomplishment em Karitiana com Descrição Complementar com sapadni 'ainda não'

Tabela 24 Interrupção de Predicados de Achievement em Karitiana com Descrição Complementar com sapadni 'ainda não'

Tabela 25 Teste da Compatibilidade com o Progressivo............................................. 114

Tabela 26 Teste da Compatibilidade com o Progressivo em Karitiana................... 115

Tabela 27 Teste da Compatibilidade com Fez o Mesmo........................................ 118

Tabela 28 Teste da Compatibilidade com o Fez o Mesmo em Karitiana................. 118 
Tabela 29 Teste da Compatibilidade com o Imperativo....................................... 122

Tabela 30 Prefixos de Concordância de Pessoa em Sentenças não Declarativas.. 122

Tabela 31 Teste da Compatibilidade com o Imperativo em Karitiana.................. 123

Tabela 32 Teste da Ambiguidade com Quase........................................................... 125

Tabela 33 Teste da Ambiguidade com Quase em Karitiana.................................... 127

Tabela 34 Comparação das Propostas para os Advérbios de Frequência.............. 144

Tabela 35 Comparação das Propostas para os Advérbios de Grau....................... 146

Tabela 36 Predicados Graduáveis e sua Relação com o Parâmetro de Comparação............................................................................................... 162

Tabela 37 Estrutura Escalar e o Parâmetro de Comparação.................................. 166

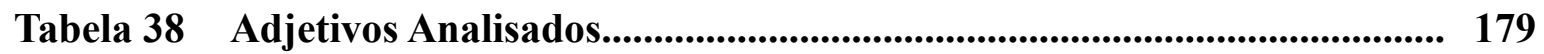

Tabela 39 Sentenças Comparativas com Adjetivos................................................ 180

Tabela 40 Adjetivos de Escala Aberta Modificados por Pita.................................. 183

Tabela 41 Adjetivos de Escala Fechada Modificados por Pita.............................. 184

Tabela 42 Proposta para o Significado de pita Modificando Adjetivos.................. 189

Tabela 43 Predicados Verbais Modificados por Pitat............................................. 194

Tabela 44 (Im)possibilidade de Adjunção de Pitat................................................. 198

Tabela 45 Pitat Modificando Predicados Atélicos (Situações Possíveis).................. 210

Tabela 46 Pitat Modificando Predicados Télicos (Pitat sem tradução)................. 213

Tabela 47 Predicados Verbais Modificados por Pitat com Negação........................ 215

Tabela 48 Relação entre Predicados Verbais e Tipos de Escalas.............................. 218

Tabela 49 Pita Modificando Substantivos................................................................. 231

Tabela 50 Substantivos Modificados por Pymbyra............................................... 233

Tabela 51 Substantivos Modificados por $\tilde{O}$ wõrã..................................................... 234

Tabela 52 Dados com muito + adjetivo e muito + verbo (Criança L)........................ 243

Tabela 53 Proposta de Tipologia dos Predicados Graduáveis............................... 245 


\title{
Forma de Apresentação dos Dados
}

Os dados do Karitiana usados nesta tese foram retirados dos corpora dos trabalhos de campo realizados durante a pesquisa com a língua Karitiana Os dados da língua utilizados nesta tese que não vieram desses corpora têm indicação de sua origem (com citação do trabalho e página).

Os dados são apresentados da seguinte forma:

\author{
(número) transcrição ortográfica com segmentação morfológica \\ glosa morfema a morfema \\ 'tradução para o português'
}

As glosas foram padronizadas de acordo com a lista apresentada abaixo. Quando utilizamos dados de outros autores, padronizamos suas glosas de acordo com a lista apresentada. Isso quer dizer que nem sempre copiamos o dado exatamente como o autor o apresenta. As glosas de um autor apenas são copiadas quando há uma discussão acerca delas, e o texto deixa claro que o dado foi inteiramente copiado, inclusive com as glosas.

Por exemplo, Carvalho $(2009,2010)$ não tem o hábito de colocar uma marca de morfema zero para concordância de terceira pessoa, quando a sentença está no modo declarativo. Estamos considerando que essa concordância está presente em todas as sentenças e estamos inserindo o morfema, mesmo nos dados tirados de Carvalho (2009, 2010). 


\section{Abreviaturas das Glosas}

1S primeira pessoa do singular

1POss primeira pessoa possessivo

2s segunda pessoa do singular

3 terceira pessoa

3ANAF terceira pessoa anafórica

ABS concordância absolutiva

ADV adverbializador

ASP aspecto

ASS assertivo

CAUS causativo

CFO contrução de foco do objeto

CIT citativo

COND condicional

COP cópula

COP.AGR concordância de cópula

DECL declarativa

DEIT deitado

DEON deôntico

DES desiderativo

DUPL duplicação

EVID evidencial

EM.PÉ em pé

IMP imperfectivo

IMPR imperativo

INT intransitivo

FUT futuro

MOV movimento

NEG negação

NFUT não futuro

NSAP não participante do ato de fala 


$\begin{array}{ll}\text { OBL } & \text { oblíquo } \\ \text { PART } & \text { particípio } \\ \text { PASS } & \text { passiva } \\ \text { PER } & \text { perfectivo } \\ \text { PL } & \text { plural } \\ \text { POS } & \text { posposição } \\ \text { SENT } & \text { sentado } \\ \text { SG } & \text { singular } \\ \text { VT } & \text { vogal temática }\end{array}$

\section{Observações}

(i) vogais epentéticas inseridas apenas por razões fonológicas aparecem entre $<>$ e não são glosadas;

(ii) quando um morfema em Karitiana corresponder a duas informações ao mesmo tempo, as glosas são associadas por um ponto final, por exemplo tyka em Karitiana é glosado por MOV.SIG;

(iii) quando uma palavra em Karitiana deve ser traduzidas por duas palavras em português, o recurso do ponto também é utilizado. Por exemplo, kanda(t) em Karitiana é traduzido por muitas.vezes.

\section{Nota sobre as traduções}

As traduções das sentenças são aquelas oferecidas pelos consultores no momento da elicitação. Outras traduções são possíveis para a sentença. Sempre que necessário, fazemos uso de uma tradução literal ou situação para explicitar o significado da sentença. Ver discussão no capítulo 2 para mais detalhes. 


\section{Outras Abreviaturas}

CP Sintagma Complementizador

IP Sintagma Flexional

VP Sintagma Verbal

DP Sintagma de Determinante

NP Sintagma Nominal

\section{Outras Convenções}

Esta tese utiliza, como recurso, o uso de itálico para palavras estrangeiras e palavras em português mencionadas no texto.

Traduções no corpo do texto são escritas entre aspas simples.

Sentenças agramaticais são marcadas com asterisco $(*)$.

Sentenças com anomalias semânticas, mas não necessariamente agramaticais são marcadas com ponto de interrogação (?) ou com cerquilha (\#). 


\section{Capítulo 1}

\section{Pano de Fundo}

\section{Introdução}

O objetivo deste capítulo é apresentar algumas características da língua Karitiana e o modelo teórico da Semântica Formal. A primeira parte, sobre a língua Karitiana, tem como propósito principal auxiliar o leitor que não está familiarizado com trabalhos sobre línguas indígenas, mais especificamente, sobre a língua Karitiana. A segunda parte, sobre a Semântica Formal, tem como objetivo auxiliar leitores que não têm experiência com pesquisas inseridas nessa teoria. Ambas as seções têm caráter introdutório e podem ser suprimidas por leitores proficientes nos respectivos temas.

\section{$1 \quad$ Karitiana}

Esta seção apresenta algumas características da língua Karitiana. O objetivo é familiarizar o leitor com os dados que serão apresentados neste trabalho. Karitiana é a única língua sobrevivente da família Arikém, uma das dez famílias do tronco Tupi. É falada por aproximadamente 320 pessoas de um grupo indígena de mesmo nome em uma reserva localizada a aproximadamente 90 quilômetros ao sul de Porto Velho, Rondônia (Storto e Vander Velden 2005) ${ }^{1}$.

Os primeiros pesquisadores que estudaram a língua Karitiana foram os membros do Summer Institute of Linguistics (SIL) David Landin e Rachel Landin, que descreveram aspectos da fonologia e da sintaxe da língua (Landin, D. 1984, 1988; Landin, R. 1982, 1987, 1989; Landin e Landin 1973). Em 1983, David Landin publicou um dicionário Karitianaportuguês e português-Karitiana (Landin, D. 1983). Nas décadas de 1980 e 1990, Daniel Everett publicou alguns artigos sobre a sintaxe da língua (Everett, D. 1985, 1993).

Luciana Storto, que pesquisa a língua Karitiana desde 1992, analisou aspectos

\footnotetext{
O dado é de 2005 e é atribuído a Nelson Karitiana (comunicação pessoal). A população Karitiana vem crescendo consideravelmente desde a década de 1970, quando a população era de aproximadamente 70 pessoas. Esse crescimento sugere que a população seja hoje maior que os 320 relatados em 2005. Everett, C. (2006), no entanto, afirma que a população é de 260 pessoas. Para uma descrição mais detalhada da população Karitiana, bem como sua distribuição entre gênero e faixa etária, ver Everett, C. (2006).
} 
fonético-fonológicos e sintáticos da língua em sua tese de doutorado em 1999 (Storto 1999). A autora tem uma vasta bibliografia sobre a língua. Algumas de suas publicações são: Storto (1994, 1996, 1998, 2001, 2002, 2003, 2005, 2008, 2010, 2011, 2012a, 2012b, 2012b, 2013, 2014); Storto e Demolin (2005) e Storto e Thomas (2012).

Mais recentemente, há a tese de doutorado de Caleb Everett sobre a fonologia e a sintaxe da língua (Everett, C. 2006) e as dissertações de mestrado de Coutinho-Silva (Coutinho-Silva 2008) e Rocha (Rocha 2011a) sobre aspectos sintáticos. Rocha tem ainda três artigos sobre a estrutura argumental dos verbos em Karitiana (Rocha 2011b, 2011c, 2012). Sobre a semântica da língua, há a minha dissertação de mestrado (Sanchez-Mendes 2009) e a dissertação de Carvalho (Carvalho 2010) e seu artigo, Carvalho (2009). Há os artigos publicados por Ana Müller (Müller, Storto e Coutinho-Silva 2006, Müller 2009, 2011, 2012, Müller e Negrão 2012) e por mim (Sanchez-Mendes 2006, 2008, 2011, 2012a, 2012b), além dos nossos trabalhos em coautoria (Müller e Sanchez-Mendes, 2008, 2009 e Sanchez-Mendes e Müller 2007).

Desse modo, pode-se dizer que a língua Karitiana é uma língua relativemente bem descrita. As características da língua Karitiana apresentadas nesta seção foram retiradas dos trabalhos acima mencionados. Os temas escolhidos dizem respeito a características gerais da língua: (i) a ordem de palavras e o padrão de concordância; (ii) a morfologia verbal; e (iii) os sintagmas nominais. Foram escolhidos esses temas e não outros por se tratar das características mais relevantes para se entender as glosas das sentenças utilizadas. A ordem de palavras das sentenças e o padrão de concordância de uma língua são as primeiras características que auxiliam na compreensão dos dados. Por isso, essas são, muitas vezes, as primeiras características apresentadas de uma língua que está sendo exposta pela primeira vez. A morfologia verbal é um tema relevante nesta tese por duas razões. Primeiramente, uma vez que os sintagmas nominais não possuem marcas morfológicas de quase nenhum tipo, muitas características linguísticas devem ser buscadas nos sintagmas verbais. Ademais, nesta tese, há um capítulo especialmente dedicado à analise semântica dos sintagmas verbais, que explora aprofundadamente esse domínio. Os sintagmas nominais constituem outro tema relevante por suas características particulares. Os sintagmas nominais em Karitiana não possuem morfologia de número, tampouco qualquer tipo de determinante.

Esses são os temas abordados na seção. A primeira subseção apresenta a ordem de palavras da língua e seu padrão de concordância. As subseções 1.2 e 1.3 apresentam a 
morfologia verbal da língua. São primeiramente apresentados os morfemas de modo, tempo e aspecto na seção 1.2; e, em seguida, na seção 1.3, a construção de cópula é discutida. Trata-se de uma construção verbal bastante comum na língua. Por fim, a seção 1.4 apresenta as características dos sintagmas nominais na língua.

\subsection{Ordem de Palavras e Padrão de Concordância}

O objetivo desta subseção é apresentar a ordem de palavras da língua Karitiana bem como o seu padrão de concordância. Karitiana é uma língua de núcleo à direita, ou seja os complementos antecedem os núcleos dos sintagmas (Storto 1999). Essa direcionalidade do núcleo está associada à existência de posposições e ao verbo na posição final em orações subordinadas. A língua Karitiana apresenta posposições, em vez de preposições. A sentença (1) ilustra essa característica. O sintagma posposicionado Porto Velho pip corresponde ao sintagma preposicionado em português para Porto Velho.

$$
\begin{aligned}
& \text { João i-tat- } \quad \text { Porto Velho pip }{ }^{2} \\
& \text { João PART-ir-ABS Porto Velho para } \\
& \text { 'O João foi para Porto Velho' }
\end{aligned}
$$

O fato de possuir núcleo à direita interfere diretamente na ordem de palavras da língua. Em sentenças não finitas, como as subordinadas, o verbo aparece in situ, no final da sentença, e a ordem de palavras é SOV ou OSV (Storto 1999). Na sentença (2) abaixo, o verbo oky 'matar' está na posição final da sentença encaixada, sem marcas de tempo e concordância. O único núcleo funcional projetado é o de aspecto. A ordem da sentença subordinada em (2) é OSV, ordem coloquialmente mais comum, segundo Storto (1999)³.

$$
\begin{array}{llll}
\text { [Boroja taso oky tykiri ] } & \varnothing \text {-naka-hyryp- } \varnothing \text { õwã. } \\
\text { cobra homem matar PERF } & \text { 3-DECL-chorar-NFUT } & \text { criança }
\end{array}
$$

'Quando o homem matou a cobra, a criança chorou'

(exemplo 4 de Storto 1999, p. 125)

A sentença (1) está em uma construção de cópula. Essa contrução será descrita em detalhes na seção 1.3.

Segundo Everett, C. (2006), no entanto, a ordem mais comum encontrada em subordinadas é SOV. Como a subordinação não faz parte diretamente do tema desta tese, vamos deixar a questão em aberto. 
Segundo Storto (1999), sentenças finitas, por outro lado, apresentam movimento obrigatório do verbo associado à presença de concordância e tempo. Nessas sentenças, os verbos ocupam a posição de núcleo de CP (Storto 1999). Karitiana possui, no entanto, uma tendência de preenchimento da posição de especificador de CP. Por isso, Storto (1999) afirma que a língua possui uma tendência $\mathrm{V}-2$, ou seja, apresenta uma propensão a exibir o verbo na segunda posição das sentenças ${ }^{4}$. Desse modo, nas sentenças matrizes, a ordem mais comum é SVO. A ordem OVS é uma ordem especial para sentenças com objeto focalizado. O exemplo (2) abaixo mostra o verbo na segunda posição com marcas de concordância e tempo em uma sentença finita típica de ordem SVO.

$$
\begin{aligned}
& \text { Taso } \quad \text { Ø-na-oky-t boroja. } \\
& \text { homem 3-DECL-matar-NFUT cobra } \\
& \text { 'O homem matou a cobra' }
\end{aligned}
$$

(exemplo 2 de Storto 1999, p. 125)

Storto (1999) utiliza a adjunção adverbial como evidência para o alçamento do verbo em sentenças matrizes. Em sentenças SVO, encontram-se três possibilidades para o aparecimento de advérbios: (i) AdvSVO (com o advérbio adjungido à esquerda de CP), (ii) SVAdvO (com o advérbio à esquerda de IP ou VP), (iii) SVOAdv (com o advérbio adjungido à direita de $\mathrm{CP})$ - representadas pelos exemplos $(4 \mathrm{a}),(4 \mathrm{~b}),(4 \mathrm{c})$ respectivamente.

a. Mynda taso Ø-na-m-potpora-j ese. vagarosamente homem 3-DECL-CAUS-ferver-FUT água

'O homem ferveu a água vagarosamente'
b. Taso
Ø-na-m-potpora-j
mynda
ese.
homem 3-DECL-CAUS-ferver-FUT vagarosamente água
'O homem ferveu a água vagarosamente'

\footnotetext{
4 O movimento obrigatório do verbo em sentenças finitas aproxima o Karitiana de línguas V-2 como o alemão. Em Alemão, no entanto não é possível o verbo aparecer na primeira posição, enquanto que Karitiana permite o verbo em posição inicial. Por isso, fala-se em tendência V-2.
} 

c. Taso Ø-na-m-potpora-j ese mynda.
homem 3-DECL-CAUS-ferver-FUT água vagarosamente
'O homem ferveu a água vagarosamente'

(exemplos 58 a 61 de Storto 1999, p. 138 e 139)

Crucialmente, o advérbio não pode ocorrer entre o sujeito e o verbo, como mostra o exemplo em (5). Isso ocorre porque essas projeções ocupadas pelo sujeito e pelo objeto nessas sentenças (espcificador de $\mathrm{CP}$ e núcleo $\mathrm{C}$ de $\mathrm{CP}$, respectivamente, estão em configuração especificador-núcleo (Storto 1999). A impossibilidade de adjunção nessa posição é uma evidência de que o sujeito e o verbo foram alçados.

$$
\begin{array}{lll}
\text { * Taso mynda } & \varnothing \text {-na-m-potpora-j } & \text { ese. } \\
\text { homem vagarosamente } & \text { 3-DECL-CAUS-ferver-FUT água } \\
\text { 'O homem ferveu a água vagarosamente' } &
\end{array}
$$

Uma vez apresentada a ordem de palavras, vamos apresentar o padrão de concordância da língua. Em Karitiana, assim como em muitas línguas Tupi, os sintagmas nominais não são normalmente marcados para caso. Na sentença (3) acima, repetida abaixo em (6), pode-se notar que nem o sujeito taso 'homem', nem o objeto boroja 'cobra' apresentam marcas de caso.

$$
\begin{aligned}
& \text { Taso } \quad \text {-na-oky-t boroja. } \\
& \text { homem 3-DECL-matar-NFUT cobra } \\
& \text { 'O homem matou a cobra' }
\end{aligned}
$$

Uma exceção possível para essa generalização é o caso oblíquo, que aparece com verbos bitransitivos, por exemplo. Uma curiosidade é que em um verbo como hit 'dar', o objeto oblíquo representa o tema, a coisa dada. A pessoa que recebe é representada pelo argumento direto. Ou seja, em Karitiana, o verbo hit 'dar' pode ser representado por algo como 'dar alguém para alguma coisa'. A sentença (7) ilustra esse fato: 
Yn Ø-naka-hit-Ø eremby-ty y-pant'in.

eu 3-DECL-dar-NFUT rede-OBL 1POSS-irmã

'Eu dei rede para a minha irmã'

Embora haja em Karitiana a marca de oblíquo, os argumentos centrais dos verbos não são marcados para caso ${ }^{5}$. Os verbos possuem, no entanto, marcas de concordância que obedecem a um padrão ergativo-absolutivo (Storto 2005). Línguas ergativo-absolutivas são línguas em que o argumento dos verbos intransitivos se assemelham com o argumento interno de verbos transitivos, e não com seu argumento externo (o agente). Em línguas nominativoacusativas, como o português e o inglês, o argumento de verbos intransitivos recebe caso nominativo. Em línguas ergativo-absolutivas, o argumento interno de verbos transitivos e o argumento de verbos intransitivos recebem caso absolutivo. $\mathrm{O}$ argumento externo de verbos transitivos recebe caso ergativo.

Em Karitiana, a concordância do verbo é com o absolutivo, ou seja, o verbo concorda com o sujeito em sentenças intransitivas e com o objeto em sentenças transitivas (Storto 2005). A sentença (8) mostra o morfema de concordância $\{y-\}$ concordando com o sujeito em primeira pessoa.

$$
\begin{array}{ll}
\text { Y-ta-opiso-t } & \text { yn. } \\
\text { 1S-DECL-ouvir-NFUT } & \text { eu }
\end{array}
$$

'Eu ouvi'

(exemplo 4 de Storto 1999, p. 161)

Em (9), embora o sujeito seja o pronome de primeira pessoa, o morfema de concordância é o de terceira pessoa $\{\mathrm{a}-\}$, concordando com o objeto.

$$
\begin{aligned}
& \text { Yn a-ta-oky-j an. } \\
& \text { eu 2S-DECL-matar-FUT você }
\end{aligned}
$$

'Eu vou matar você'

(exemplo 1 de Storto 1999, p. 157)

\footnotetext{
5 Os argumentos centrais seriam o agente e o tema, representados pelo nominativo e pelo acusativo, respectivamente, nas línguas de padrão nominativo-acusativo de concordância. Observa-se, no entanto, que em Karitiana, o tema é marcado por caso oblíquo em sintagmas verbais bitransitivos, como mostrou o dado em (5).
} 
O padrão de concordância exibido pela língua está de acordo com a classificação de seus verbos segundo Rocha (2012). Segundo o autor, todos os verbos intransitivos em Karitiana são inacusativos. Os verbos intransitivos são divididos em dois tipos pela literatura: os inergativos e os inacusativos. Os verbos inergativos são os verbos considerados como intransitivos canônicos, seu sujeito ocupa a posição de argumento externo, assim como o sujeito de verbos transitivos. Exemplos de verbos inergativos em português são trabalhar, sorrir e dançar. Verbos inacusativos são verbos em que o sujeito sintático não possui características de agente. Esses verbos possuem apenas argumento interno (Burzio 1986). Chegar, morrer e cair são exemplos de verbos inacusativos em português.

Rocha (2012) mostra que todos os verbos intransitivos do Karitiana podem alternar como verbos transitivos, o que demonstra que eles são todos inacusativos, uma vez que apenas intransitivos inacusativos poderiam sofrer esse processo de alternância. Os dados em (10) e (11) ilustram essa possibilidade. Em (10a), o verbo otam 'chegar' é utilizado como intransitivo. Em (10b) o verbo está transitivizado pelo morfema causativo $\{-m-\}$. A alternância não é inesperada, uma vez que o verbo chegar é inacusativo em línguas como o inglês e o português. Já o dado em (11) mostra que mesmo um verbo tradicionalmente considerado como inergativo, como terekterek 'dançar' e, portanto, não alternante; pode ser transitivizado em Karitiana.

a. $\quad$-pyry-otam- $<$ y $>$-n João.

3-ASS-chegar-NFUT João

'O João chegou'

(exemplo 5 de Rocha 2012, p. 166)

b. Ø-pyry-m-otam-<y>-n João yn.

3-ASS-CAUS-chegar-NFUT João eu

'Eu fiz o João chegar'

(exemplo 7 de Rocha 2012, p. 167) 
(11)

a. $\quad \varnothing$-py-terekterek-a-n taso.

3-ASS-dançar-VT-NFUT homem

'O homem dançou'

(exemplo 9 de Rocha 2012, p. 167)

b. Y-py-m-terekterek-a-n hyrỹj hãrã.

1-ASS-CAUS-dançar-VT-NFUT música boa

'A boa música me fez dançar'

(exemplo 12 de Rocha 2012, p. 168)

Rocha (2011a, 2012) utiliza dados como os expostos acima para mostrar que todos os verbos intransitivos têm o mesmo comportamento sintático, no que diz respeito à alternância. Porém, esses dados mostram ainda uma outra característica da língua: uma vez que todos os verbos intransitivos são considerados sintaticamente como inacusativos, isso indica que os verbos intransitivos em Karitiana possuem como argumento sempre um argumento interno. Desse modo, é possível concluir que em Karitiana, a concordância se dá sempre com o argumento interno do verbo, que é representado pelo de sujeito sintático nos verbos intransitivos, e pelo objeto nos verbos transitivos.

Não há consenso, no entanto, se as marcas de pessoa que aparecem no verbo são mesmo marcas de concordância. Landin, D. (1984) e Everett, C. (2006) defendem que as marcas de pessoa no verbo seriam pronomes cliticizados e não morfemas de concordância, como defende Storto (2005). Storto (2005) apresenta dois argumentos em favor de sua análise. Primeiramente, se essas marcas fossem pronomes cliticizados, não se esperaria a sua coocorrência com pronomes plenos na sentença. Como é possível notar nos dados (8) e (9), acima, repetidos aqui em (12) e (13), não é esse o caso.

$$
\text { Y-ta-opiso-t yn. }
$$

1S-DECL-ouvir-NFUT eu

'Eu ouvi'

(exemplo 4 de Storto 1999, p. 161) 
Yn a-ta-oky-j an.

eu 2S-DECL-matar-FUT você

'Eu vou matar você'

(exemplo 1 de Storto 1999, p. 157)

Além disso, como se viu, sentenças subordinadas, que são sentenças com verbo in situ, aparecem sem essas marcas. Isso indica que esses são mesmo morfemas inseridos por um núcleo funcional que expressam concordância de pessoa. Com base nesses argumentos, assumiremos a proposta de Storto (2005) de que as marcas de pessoa infixadas nos verbos são morfemas de concordância.

A tabela 1, abaixo, apresenta os pronomes pessoais e morfemas de concordância de pessoa em Karitiana, quando as sentenças estão no modo declarativo ${ }^{6}$.

Tabela 1: Prefixos de Concordância de Pessoa em Karitiana

\begin{tabular}{|l|c|c|}
\hline \multicolumn{1}{|c|}{ PESSOA } & PRONOME & MORFEMA \\
\hline $1^{\mathrm{a}}$ SG & yn & y- \\
\hline $2^{\mathrm{a}}$ SG & an & a- \\
\hline $1^{\mathrm{a}}$ PL inclusiva & yta & yj- \\
\hline $1^{\mathrm{a}}$ PL exclusiva & yjxa & yta- \\
\hline $2^{\mathrm{a}}$ PL & ajxa & aj- \\
\hline $3^{\mathrm{a}}$ pessoa (SG ou PL) & i & $\varnothing-$ \\
\hline
\end{tabular}

Em Karitiana, a terceira pessoa não possui diferença entre singular e plural. O pronome é sempre $i$ e o morfema de concordância é sempre $\left\{\varnothing_{-}\right\}$nas sentenças declarativas. Em Karitiana há dois tipos de primeira pessoa do plural. A primeira pessoa do plural inclusiva é aquela que inclui o ouvinte. Nós inclusivo é a soma da primeira pessoa com a(s) segunda(s) e terceira(s) pessoas. A primeira pessoa exclusiva, por outro lado, exclui o interlocutor. Nós exclusivo é a soma da primeira pessoa com uma(s) terceira(s) pessoa(s), excluindo a(s) segunda(s) pessoa(s). Essa distinção é comum em muitas línguas das Américas (Tupi e Quéchua, por exemplo) e da Oceania.

Esta seção apresentou a ordem de palavras e o padrão de concordância da língua Karitiana. Em Karitiana a ordem de palavras depende do tipo de sentença considerada. Em sentenças subordinadas, é possível observar o verbo na posição final, em que é gerado. Em sentenças matrizes finitas, por outro lado, observa-se uma ordem derivada por movimento do

\footnotetext{
Para marcas de pessoa em outras construções, ver Storto (2002).
} 
verbo. Nessas sentenças, a ordem mais comum é SVO no modo declarativo. Quanto ao padrão de concordância, embora os argumentos centrais em Karitiana não sejam marcados para caso, a concordância verbal apresenta um padrão ergativo-absolutivo em que há concordância com o argumento interno do verbo, o argumento absolutivo. A próxima subseção tratará de outras marcas flexionais nos verbos como modo, tempo e aspecto.

\subsection{Modo, Tempo e Aspecto}

O objetivo desta subseção é apresentar as categorias de modo, tempo e aspecto em Karitiana. A primeira categoria a ser discutida será a de modo. Como ficará claro ao longo da seção, essa classe de morfemas não é analisada de forma consensual pelos autores que a estudaram. Em seguida, será apresentada uma breve descrição da categoria de tempo na língua e, em seguida, da de aspecto.

\subsubsection{Modo}

A língua Karitiana apresenta uma gama de morfemas que foram descritos como morfemas de modo em Storto (2002): declarativo, assertivo, citativo, deôntico, condicional e imperativo, apresentados em (14a) a (14f), respectivamente.
a. Taso Ø-na-oky-t
boroja.
$=(3)=(6)$
homem 3-DECL-matar-NFUT cobra
'O homem matou a cobra'

(exemplo 2 de Storto 1999, p. 125)

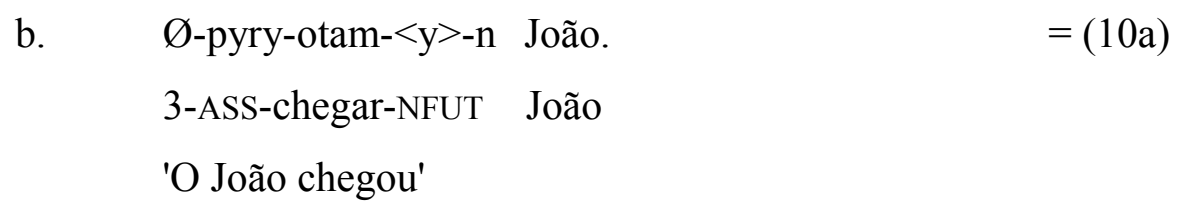

(exemplo 5 de Rocha 2012, p. 166) 
c. [Kinda pojongo ta-ket ta-m-tata]-ty iri-kãra-j Botyj. coisa estrangeira 3ANAF-irmão 3ANAF-CAUS-ir-OBL CIT-pensar-FUT Deus 'Deus pensou que seu irmão tranformou-se em um estrangeiro'

(exemplo 2 de Storto 2002, p. 157)

d. Pyn-pyt'y-t.

DEON-comer-NFUT

'Ele deve comer'

(exemplo 1 de Storto 2002, p. 157)

e. Yn jy-pit yn ip an ti-'y-t $t^{7}$.

eu COND-pegar eu peixe você CFO-comer-OBL

'Eu ia pegar um peixe para você comer'

(exemplo 2 de Storto 2002, p. 158)

f. A-pongyw-a.

2S-quieto-IMPR

'Cale-se'

(exemplo 3 de Storto 2002, p. 158)

A tabela abaixo resume os morfemas de modo aprensentados em Storto (2002):

Tabela 2: Morfemas de Modo em Karitiana

\begin{tabular}{|l|c|}
\hline \multicolumn{1}{|c|}{ MODO } & MORFEMA \\
\hline Declarativo & $\mathrm{na}(\mathrm{ka})-/ \mathrm{ta}(\mathrm{ka})-$ \\
\hline Assertivo & pyt $<\mathrm{y}>-$ \\
\hline Citativo & iri- \\
\hline Deôntico & pyn- \\
\hline Condicional & $\tilde{\mathrm{jy}}-$ \\
\hline Imperativo & $-\mathrm{a} /-\varnothing$ \\
\hline
\end{tabular}

$7 \quad P y t^{\prime} y$ e 'y são duas palavras usadas para 'comer'. ' $y$, na verdade, pode ser considerado como tendo um significado mais amplo, como 'ingerir'. Pyt'y é o verbo específico para ingestão de sólidos, enquanto que $s e^{\prime} y$ 'beber' é usado para a ingestão de líquidos. 
Como o foco dessa pesquisa não está no modo, os dados desta tese estão em sua grande maioria no modo que autora chama de declarativo, o modo default da língua, que é expresso pelos morfemas $\{n a(k a)-\} /\{t a(k a)-\}$ prefixados às raízes verbais. A sílaba $\{-k a\}$ é um morfema chamado de aumentativo por Landin, D. (1984) que ocorre quando as raízes verbais têm a primeira sílaba acentuada (Landin, D. 1984, Everett, C. 2006, Storto 2008). $\{n a-\}$ e $\{n a k a-\}$ são, portanto, alomorfes; assim como $\{t a-\}$ e $\{t a k a-\}$. As formas mais curtas $\{n a-\}$ e $\{t a-\}$ são usadas quando a primeira sílaba do verbo não é acentuada e $\{n a k a-\}$ e $\{t a k a-\}$ são usados quando a primeira sílaba do verbo é acentuada.

$\{N a(k a)-\}$ e $\{t a(k a)-\}$ são ainda alomorfes um do outro. $\{N a(k a)-\}$ é o usado quando a concordância é de terceira pessoa, expressa pelo morfema zero, tanto no singular, quanto no plural. O morfema $\{t a(k a)$ - $\}$ é utilizado com os outros morfemas de pessoa.

No exemplo (15), abaixo, a concordância é com a primeira pessoa, logo o morfema selecionado é $\{t a(k a)-\}$. O verbo tat 'ir' é uma sílaba acentuada e, por isso, utiliza-se o morfema $\{$ taka- $\}$.

$$
\begin{aligned}
& \text { Y-taka-tat- } \varnothing . \\
& \text { 1S-DECL-ir-NFUT }
\end{aligned}
$$

'Eu fui'

(exemplo 5 de Storto 2008, p. 186)

Em (16), a concordância é de terceira pessoa, com o objeto pikom 'macaco', então o morfema selecionado é $\{n a(k a)-\}$. A primeira sílaba do verbo oky 'ir' não é acentuada e, por isso, o morfema declarativo utilizado é $\{n a-\}$.

$$
\begin{aligned}
& \text { Yn } \varnothing \text {-na-oky-t pikom. } \\
& \text { eu 3-DECL-matar-NFUT macaco } \\
& \text { 'Eu matei macaco' }
\end{aligned}
$$

(exemplo 6 de Storto 2008, p. 187)

Os morfemas $\{n a(k a)-\} /\{t a(k a)-\}$ não são tratados por Everett, C. (2006) como morfemas de modo, mas como marcas de construções de participante de ato de fala (do inglês Speech Act Participant). Segundo o autor, essas construções formam um dos tipos de vozes 
básicas da língua. $\mathrm{O}$ autor afirma que, embora esses morfemas apareçam quase sempre em sentenças declarativas, esse não é sempre o caso.

$\mathrm{O}$ argumento do autor para rejeitar análise de que esses morfemas sejam de modo declarativo é a sua coocorrência com o morfema de evidencial indireto. Morfemas evidenciais são morfemas que codificam gramaticalmente a maneira como o falante obteve a informação enunciada. Eles podem indicar, por exemplo, se a pessoa viu o que ela está afirmando ou se ela ouviu de alguém a informação. Em Karitiana, o morfema \{-saryt $\}$ indica que a informação veiculada possui evidências indiretas, por exemplo, o falante ouviu dizer $^{8}$. O dado (17) ilustra esse morfema.

$$
\begin{aligned}
& \text { I } \varnothing \text {-naka-pydn-saryt- } \varnothing \text { bola. } \\
& 3 \text { 3-DECL-chutar-EVID-NFUT bola } \\
& \text { 'Ele chutou a bola' } \\
& \text { Situação: eu ouvi ele chutar a bola }{ }^{10}
\end{aligned}
$$

(exemplo 12.102 de Everett, C. 2006, p. 282)

Segundo Everett, C. (2006), o modo declarativo está associada a um alto grau de certeza do falante. Em línguas como Patwin, língua da região do norte da Califórnia, nos Estados Unidos, por exemplo, os morfemas de declarativo e de evidencial indireto são mutualmente excludentes. Everett, C. (2006) defende que a possibilidade de ocorrência da marcas de evidencial $\{$-saryt $\}$ com os morfemas $\{n a(k a)-\} /\{t a(k a)-\}$ indica que que eles não devem ser analisados como marcas de modo declarativo, como em Storto (1999). O autor ressalva que esses prefixos ocorrem frequentemente em sentenças declarativas e que há uma forte associação entre essas marcas e a semântica de sentença declarativa. No entanto, essa relação não é biunívoca, não estando presente em todas as sentenças com $\{n a(k a)-\} /\{t a(k a)-\}$.

Embora o argumento do autor para rejeitar a análise de que $\{n a(k a)-\} /\{t a(k a)-\}$ sejam morfemas de modo declarativo seja pertinente, a sua proposta de análise alternativa não

\footnotetext{
Esse morfema contrasta com o morfema de evidencial direto \{-ta'ãt $\}$ que veicula que o falante viu a ocorrência relatada. Ver Storto (2002) para mais detalhes.

9 As glosas não foram copiadas de Everett, C. (2006). O autor não adiciona o morfema zero de concordância com a terceira pessoa e glosa $\{n a k a-\}$ como NSAP, de Speech Act Participant. Como ficará claro mais abaixo, adotaremos a proposta de Storto (2002) e glosaremos $\{n a(k a)-\} /\{t a(k a)-\}$ como $D E C L$, de declarativo.

10 A tradução da sentença foi modificada do dado retirado de Everett, C. (2006). A tradução oferecida pelo autor é 'He kicked the ball, I heard' ('Ele chutou a bola, ou ouvi'). No entanto, essa não é uma boa tradução para a sentença, pois ela mistura a tradução da sentença com as informações semânticas do morfema, dando a ilusão de que a sentença em Karitiana apresenta uma coordenação de orações.
} 
tem uma capacidade explicativa melhor.

Everett, C. (2006) defende que esses morfemas expressam uma voz em Karitiana, que ele denomina de participante do ato de fala (Speech Act Participant). Na verdade $\left\{n a(k a)_{-}\right\}$e $\{t a(k a)-\}$ representariam duas vozes da língua. $\{t a(k a)-\}$ representaria a voz de participante de ato de fala (SAP - Speech Act Participant), enquanto que $\{n a(k a)-\}$ representaria o não participante do ato de fala (NSAP - Non-Speech Act Participant). Os morfemas recebem esse nome porque, como já foi descrito anteriormente, $\{n a(k a)-\}$ é usado quando o argumento absolutivo é de terceira pessoa, logo, uma pessoa que não participa do ato de fala e $\{t a(k a)$ - $\}$ é utilizado quando a concordância é com a primeira e a segunda pessoa, ou seja, as pessoas participantes do ato de fala.

A proposta do autor leva em conta a definição de voz de Shibatani (2006) (apud Everett, C. 2006). Trata-se de uma abordagem baseada em dados tipológicos e de orientação linguistico-cognitiva. Mais especificamente, o autor utiliza a definição de voz que faz uma associação entre a ação eventiva e seus participantes. Basicamente, o autor traduz as vozes SAP e NSAP da seguinte forma:

a. Quando o evento descrito termina dentro de cena do discurso, ou seja, em um participante do ato de fala (SAP), então, o prefixo é $\{t a(k a)-\}$;

b. Quando o evento termina fora da cena do discurso, ou seja, em um não participante do ato de fala (NSAP), então o prefixo é $\{n a(k a)-\}$.

Logo, pode-se observar que se trata apenas de uma mudança de nomenclatura para a descrição do mesmo fenômeno. Dizer em (18a) que $\{t a(k a)-\}$ é utilizado quando o evento descrito termina dentro da cena do discurso é o mesmo que dizer que ele é o morfema para a primeira e a segunda pessoas. Assim como quando o evento termina fora da cena do discurso (18b) trata-se da terceira pessoa.

Essa tradução do uso de $\{n a(k a)-\} /\{t a(k a)-\}$ como voz está longe de ser incontroversa. O próprio autor assume que esse não é o uso canônico da noção de voz e que ele está utilizando uma definição pouco usual para o fenômeno (de Shibatani 2006 apud Everett, C. 2006). O que se nota, é que a mudança de rótulo de modo declarativo para voz de participante (ou não) do ato de fala não tem poder explicativo.

A assunção de que os prefixos $\{n a(k a)-\} /\{t a(k a)-\}$ sejam modo declarativo ainda traz problemas, mas não há um esclarecimento maior em considerar esses morfemas como marcas 
de voz. Logo, neste trabalho, os morfemas $\{n a(k a)-\} /\{t a(k a)-\}$ serão glosados como modo declarativo, com a ressalva feita nesta seção de que esse tópico ainda merece bastante atenção.

As sentenças no modo declarativo apresentam a seguinte sequência de morfemas verbais de concordância, modo e tempo: PESSOA-MODO.DECLARATIVO-verbo-TEMPO. O exemplo em (19) ilustra esse estrutura.
Taso Ø-na-oky-t
boroja.
homem 3-DECL-matar-NFUT cobra
'O homem matou a cobra'

$=(3)=(6)=(14)$

(exemplo 2 de Storto 1999, p. 125)

Esta seção discutiu os morfemas de modo declarativo na línguas Karitiana. A próxima seção apresentará brevemente os morfemas de tempo nas construções verbais declarativas.

\subsubsection{Tempo}

Em Karitiana os morfemas de tempo são futuro e não futuro (Storto 2002). Segundo Everett, C. (2006), esse sistema de divisão do tempo é relativamente raro nas línguas do mundo, embora seja atestado, por exemplo, em Hua, uma língua da Nova Guiné e em Wari, uma língua amazônica. Em Karitiana, os morfemas para futuro e não futuro variam em sentenças afirmativas de acordo com o modo empregado (Storto 2002 e Everett, C. 2006).

Neste trabalho, os dados aparecem na construção verbal default da língua, que foi descrita acima como modo declarativo (seguindo a proposta de Storto 2002). Em sentenças no modo declarativo, o sufixo de tempo não futuro é $\{-t\}$, quando o verbo termina com vogal, exemplo em (20); e $\{-\varnothing\}$, quando termina com consoante, exemplo em (21).
Taso Ø-naka-'y-t
kinda'o.
homem 3-DECL-comer-NFUT fruta
'O homem come/comeu fruta' 
(21)
João Ø-na-pimbik- Ø
gooj $^{11}$.
João 3-DECL-empurrar-NFUT carro
'O João empurra/empurrou carro'

O sufixo de futuro é $\{-j\}$ se o verbo termina em vogal, exemplo em (22); e $\{-i\}$ se o verbo termina em consoante - sentença (23).
Taso $\quad \varnothing$-na-oky-j boroja.
homem 3-DECL-matar-FUT cobra
'O homem vai matar a cobra'

(23) Y-taka-tat-i yn.

1S-DECL-ir-FUT eu

'Eu irei'

(exemplo 12.70 de Everett, C. 2006, p. 269)

A tabela 3 resume a distribuição desses morfemas.

Tabela 3: Sufixos de Tempo do Karitiana no Modo Declarativo

\begin{tabular}{|l|c|c|}
\hline \multicolumn{1}{|c|}{ Restrição } & Futuro & Não Futuro \\
\hline se o verbo termina em vogal & $\{-j\}$ & $\{-t\}$ \\
\hline se o verbo termina em consoante & $\{-i\}$ & $\{-\emptyset\}$ \\
\hline
\end{tabular}

O tempo não futuro descreve situações no passado e no presente. A interpretação default, no entanto, é de um evento ocorrido no passado (Everett, C. 2006). A próxima seção discutirá os morfemas de aspecto em Karitiana. Nela, serão apresentadas algumas estratégias que a língua possui para descrever situações no presente e habituais.

$\overline{11}$ Em Karitiana, a palavra utilizada para 'carro' é gooj, a mesma paravra utilizada para 'canoa'. 


\subsubsection{Aspecto}

A língua Karitiana possui uma variedade de marcas que expressam aspecto. Storto (2002) descreveu auxiliares de aspecto imperfectivo e referencial e clíticos de aspecto repetitivo, inceptivo e dubitativo. Esta subseção apresenta especificamente os morfemas de imperfectividade na língua. O que há de singular nessas marcas é que elas possuem uma complexidade semântica e carregam além do significado imperfectivo, informações de número de participantes do evento e de sua posição (Landin, D. 1984, Storto 2002, Everett, C. 2006 e Carvalho 2009, 2010).

O exemplo em (24) mostra que o marcador aspectual tyka apresenta informações de imperfectivo e de que o sujeito é singular e está em movimento.

$$
\begin{aligned}
& \text { Maria } \varnothing \text {-naka-'y ty-ka-t kinda'o. } \\
& \text { Maria 3-DECL-comer IMP-MOV.SG-NFUT fruta } \\
& \text { 'Maria está comendo fruta (em movimento)' }
\end{aligned}
$$

(exemplo 40a de Carvalho 2010, p. 45)

Landin, D. (1984) e Everett, C. (2006) defendem que as marcas de aspecto são sufixos verbais que ocorrem entre a raiz verbal e morfema de tempo ${ }^{12}$. Storto (2002) e Carvalho $(2009,2010)$, por outro lado, afirmam que se trata de um verbo auxiliar. Os argumentos a favor dessa última análise são: (i) os marcadores auxiliares ocorrem com os mesmos morfemas de tempo que ocorrem em verbos e; (ii) eles possuem um acento próprio, configurando portanto uma palavra, e não um morfema preso (Carvalho 2009, 2010).

Assumiremos a proposta de Storto (2002) e Carvalho $(2009,2010)$ e consideraremos os marcadores aspectuais como verbos auxiliares. No entanto, o primeiro argumento apresentado pelas autoras pode ser questionado uma vez que o marcador aspectual não apresenta todos os morfemas flexionais que os verbos plenos possuem. $\mathrm{O}$ único morfema que aparece é o de tempo; ele não apresenta morfemas de modo ou pessoa, como os verbos plenos.

Segundo Landin, D. (1984), Storto (2002), Everett, C. (2006) e Carvalho (2009, 2010), os auxiliares aspectuais são bimorfêmicos. O primeiro morfema indica imperfectivo e

\footnotetext{
12 Segundo Carvalho $(2009,2010)$ o morfema de tempo pode aparecer também coocorrendo na raiz verbal e na marca de aspecto.
} 
o segundo está associado à posição do sujeito da ação. Desse modo, tyka é dividido em $\{t y$ - $\}$, morfema de imperfectivo e $\{-k a\}$, marcador de movimento do sujeito. A tabela 4, abaixo, apresenta a decomposição dos quatro marcadores de imperfectivo em Karitiana (Landin, D. 1984, Storto 2002, Everett, C. 2006 e Carvalho 2009, 2010):

Tabela 4: Morfemas de Imperfectivo em Karitiana

\begin{tabular}{|c|l|l|}
\hline $\begin{array}{c}\text { Auxiliares } \\
\text { Imperfectivos }\end{array}$ & \multicolumn{2}{|c|}{$\begin{array}{c}\text { Decomposição } \\
\text { Morfêmica }\end{array}$} \\
\hline \multirow{2}{*}{ tyka } & $\{t y-\}$ & imperfectivo \\
\cline { 2 - 3 } & $\{-k a\}$ & movimento \\
\hline \multirow{2}{*}{ tyja } & $\{t y-\}$ & imperfectivo \\
\hline & $\{-j a\}$ & sentado \\
\hline \multirow{2}{*}{ tyso } & $\{t y-\}$ & imperfectivo \\
\cline { 2 - 3 } & $\{-s o\}$ & em pé \\
\hline \multirow{2}{*}{ tysyp } & $\{t y-\}$ & imperfectivo \\
\cline { 2 - 3 } & $\{-s y p\}$ & deitado \\
\hline
\end{tabular}

Os exemplos (25), (26) e (27), abaixo, mostram sentenças com tyja, tyso e tysyp, respectivamente.

Maria Ø-naka-'y ty-ja-t kinda'o.

Maria 3-DECL-comer IMP-SENT-NFUT fruta

'Maria está comendo fruta (sentada)'

(exemplo 40b de Carvalho 2010, p. 46)

(26) Maria Ø-naka-'y ty-so-t kinda'o.

Maria 3-DECL-comer IMP-EM.PÉ-NFUT fruta

'Maria está comendo fruta (em pé)'

(exemplo 40c de Carvalho 2010, p. 46) 
Maria Ø-naka-'y ty-syp-Ø kinda'o.

Maria 3-DECL-comer IMP-DEIT.PL-NFUT fruta

'Maria está comendo fruta (deitada)'

(exemplo 40d de Carvalho 2010, p. 46)

Everett, C. (2006) chama atenção para o fato de que o aspectual tysyp parece não possuir mais a informação de que a posição do nominal é deitada. Segundo o autor, essa distinção de posição estaria se perdendo na língua. Muitos falantes jovens não veem diferença de interpretação nos dados de (25) a (27) acima.

O que é digno de nota a respeito da variação de acordo com a posição é que ela diz respeito sempre ao sujeito da sentenças, seja o seu verbo intransitivo ou transitivo. Nesse sentido esse é um fenômeno que se aplica apenas ao argumento nominativo (Landin, D. 1984 e Everett, C. 2006). Essa distribuição não estaria de acordo com o padrão de concordância da língua que seleciona a distribuição ergativo-absolutiva. Logo, o esperado é que houvesse uma distribuição com o sujeito intransitivo e objeto transitivo de um lado e o sujeito transitivo de outro. Nesse caso, é possível afirmar que, na língua Karitiana, alguns fenômenos obedecem a distribuição ergativo-absolutiva, como a concordância verbal, por exemplo; e outros, como a distribuição do morfema posicional dos auxiliares aspectuais, obedecem a um padrão nominativo-acusativo.

Outra característica digna de nota acerca dos marcadores aspectuais em Karitiana é que a sua distribuição em sentenças no futuro e no não futuro não é consensual. Everett, C. (2006) e Carvalho $(2009,2010)$ discordam quanto à relação entre o marcador de imperfectivo e os morfemas de tempo. Everett, C. (2006) afirma que as sentenças com o marcadores aspectuais não são possíveis no tempo futuro. Ele ilustra sua tese com o dado em (28). Como se vê, a sentença com o marcador $\{$ tyja $\}$ de imperfectivo e com sufixo verbal $\{-j\}$ de futuro é agramatical.

$$
\begin{aligned}
& \text { *I } \quad \text { Ø-na-oky ty-ja-j } \quad \text { saara. } \\
& \text { ele } 3 \text {-DECL-matar IMP-SENT-FUT jacaré } \\
& \text { 'Ele vai estar matando jacaré (sentado)' }
\end{aligned}
$$

(exemplo 12.87 de Everett, C. 2006, p. 277) 
Carvalho $(2009,2010)$, no entanto, mostra que um outro auxiliar, tyka é compatível apenas com sentenças em que o tempo de tópico é posterior ao tempo de enunciação da sentença. Nesse caso, ela defende a impossibilidade de uso desse marcador com sentenças que descrevem situações no passado. A sentença em (29) ilustra esse fato.

* Koot yn Ø-naka-m-'a ty-ka-t gooj.

ontem eu 3-DECL-CAUS-fazer IMP-MOV.SG-NFUT canoa

'Ontem eu estava fazendo canoa'

(exemplo 98b de Carvalho 2010, p. 92)

Segundo Carvalho (2010), não há incompatibilidade do auxiliar aspectual tyka com o tempo futuro, como mostra o exemplo em (30):
Um dia yn $\varnothing$-naka-m-'a
ty-ka-j
gooj.
um dia eu 3-DECL-CAUS-fazer IMP-MOV.SG-FUT canoa
'Um dia (no futuro) eu estarei fazendo canoa'

(exemplo 98c de Carvalho 2010, p. 92)

A discordância entre Everett, C. (2006) e Carvalho (2010) quanto à coocorrência da aspecto imperfectivo com tempo futuro pode ser devida ao marcador escolhido para os testes. O exemplo que Everett, C. (2006) fornece é com o marcador tyja, o imperfectivo que marca a posição sentado. Carvalho (2010) concentra sua análise em tyka, o imperfectivo que marca movimento. Nota-se, portanto, que a distribuição e o significado dos auxiliares aspectuais em Karitiana é um tópico que merece uma investigação mais refinada.

Uma outra propriedade dos auxiliares aspectuais em Karitiana é que eles apresentam uma restrição relacionada ao número (singular/plural) de participantes da ação. Everett, C. (2006) mostra que o marcador tysyp só pode ser utilizado quando o nominal absolutivo é plural. Segundo o autor, sentença (31), por exemplo, só pode ser usada em contextos em que há mais de uma onça. 


$$
\begin{aligned}
& \text { Ombaky i-pyt'y ty-syp-Ø } \\
& \text { onça PART-comer IMP-DEIT.PL-NFUT } \\
& \text { 'As onças estão comendo' }
\end{aligned}
$$

Carvalho (2010), no entanto, mostra que um outro marcador aspectual, o tyka, pode ser usado somente em sentenças em que o sujeito é singular. Essa é mais uma característica que se aplica apenas ao argumento nominativo. Segundo a autora, o dado em (32) abaixo só pode ser usado em contextos em que há apenas uma criança, não sendo adequado para descrever um contexto em que há mais de uma criança.

$$
\begin{aligned}
& \text { Ø-na-ohit ty-ka-t õwã. } \\
& \text { 3-DECL-pescar IMP-MOV.SG-NFUT criança } \\
& \text { 'A criança está pescando' }
\end{aligned}
$$

Já no dado em (33), em que há um sujeito plural, a sentença fica agramatical com o auxiliar tyka.

$$
\begin{aligned}
& \text { (33) * João Pedro Ø-na-manga ty-ka-t } \quad \text { Rex. } \\
& \text { João Pedro 3-DECL-levantar IMP-MOV.SG-NFUT Rex } \\
& \text { 'O João e o Pedro estão levantando o Rex' }
\end{aligned}
$$

(exemplo 103b de Carvalho 2010, p. 98)

Esta subseção apresentou os auxiliares aspectuais em Karitiana. Há uma gama de marcadores de aspecto que marcam imperfectividade (descritos como progressivos por alguns autores). Sua restrição a situações no tempo passado ou futuro ainda é objeto de controvérsia na literatura. Há quem defenda que um deles (tyja) não pode ser usado em sentenças no tempo futuro (Everett, C. 2006) e quem defenda que um deles (tyka) não pode aparecer em sentenças que descrevem um evento passado (Carvalho 2010). Além disso, a sua restrição de uso quanto ao número dos argumentos nominais envolvidos também não é consensual. Eles ora indicam número plural do argumento absolutivo (tysyp descrito em Everett, C. 2006) ora indicam 
singular do argumento nominativo (tyka descrito em Carvalho 2010). Esta subseção fecha a seção sobre os morfemas funcionais nas construções verbais em Karitiana. A próxima seção tratará de uma construção verbal bastante comum na língua, a construção de cópula.

\subsection{Construção de Cópula}

O objetivo desta seção é apresentar a construção de cópula em Karitiana. Optou-se por descrevê-la em uma seção separada porque se trata de uma construção verbal bastante comum na língua que ocorrerá em boa parte dos dados tratados nesta tese. O dado em (34) abaixo mostra uma sentença com construção de cópula típica.

$$
\begin{aligned}
& \text { Taso } \quad \text {-na-aka-t i-se'a-t. } \\
& \text { homem 3-DECL-COP-NFUT PART-bom-ABS } \\
& \text { 'O homem é bom' }
\end{aligned}
$$

(exemplo 5 de Storto 2010, p. 2)

No entanto, esse tipo de construção não ocorre apenas em predicação com adjetivos. Everett, C. (2006) e Storto (2010) concordam que essa construção pode ocorrer com predicados nominais, adjetivais e verbais. As sentenças abaixo apresentam a construção com o predicado nominal kinda'o 'fruta' e o predicado verbal kat 'dormir'.

$$
\begin{aligned}
& \text { Byyty } \varnothing \text {-na-aka-t kinda'o-t. } \\
& \text { mamão 3-DECL-COP-NFUT fruta-ABS } \\
& \text { 'Mamão é fruta' }
\end{aligned}
$$

(exemplo 1 de Storto 2010, p. 2)
(36)
Taso $\quad$ i-kat-t. homem 3-DECL-COP-NFUT PART-dormir-ABS
'O homem dormiu'

(exemplo 7 de Storto 2010, p. 2) 
O uso com predicados verbais é o mais intrigante, uma vez que não se trata de uma predicação copular no seu sentido mais tradicional, como no caso do adjetivo e do substantivo. Além disso, não é qualquer tipo de verbo que pode aparecer em construções desse tipo; apenas os verbos intransitivos. A sentença em (37) mostra que uma sentença com um verbo transitivo como ' $y$ 'comer' em uma construção de cópula é agramatical.

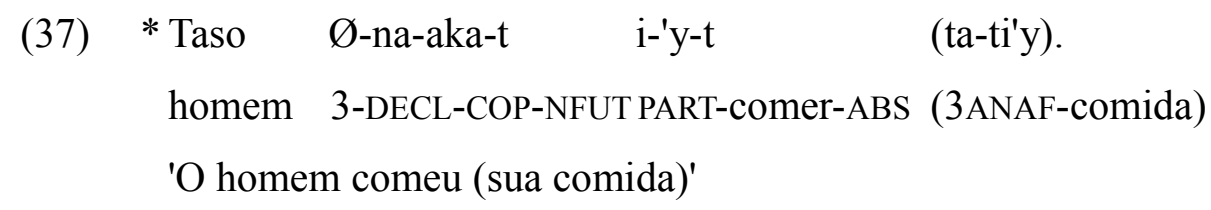

(exemplo 10 de Storto 2010, p. 3)

Storto (2010) analisa as sentenças copulares em Karitiana como sentenças bioracionais. A predicação copular naakat pode aparecer com substantivos, adjetivos ou verbos intransitivos. O verbo de cópula $a k a$ seleciona uma minioração nominalizada como complemento, por isso a presença do prefixo $\{i-\}$ de particípio quando o complemento é um verbo ou adjetivo. Essas sentenças são entendidas como sentenças de alçamento do sujeito da minioração para a posição pré-copular, uma posição de foco. O sufixo $\{-t\}$ ou $\{-\varnothing\}$ que aparece na minioração é uma marca morfosintática desse movimento do sujeito. Por isso, ele foi glosado como concordância absolutiva por Storto (2008, 2010). Sintaticamente, a estrutura da cópula é a seguinte:

$$
\begin{aligned}
& \text { Sujeito }_{i} \text { naakat }\left[\text { nom }\left[\text { мо } t_{i} X\right]\right] \\
& \text { onde } X \text { pode ser um substantivo, um adjetivo ou um verbo intransitivo }
\end{aligned}
$$

(representação 13a de Storto 2010, p. 3)

A tabela 5, abaixo, apresenta uma generalização a respeito das sentenças copulares. Ela mostra ainda quais são os morfemas que figuram em cada uma delas. 
Tabela 5: Generalizações sobre a Construção de Cópula

\begin{tabular}{|l|l|}
\hline $\begin{array}{c}\text { Núcleo do Complemento } \\
\text { da Cópula }\end{array}$ & \multicolumn{1}{c|}{ Construção } \\
\hline Substantivo & naakat + substantivo-ABSOLUTIVO \\
\hline Adjetivo & naakat + PARTICÍPIO-adjetivo-ABSOLUTIVO \\
\hline Verbo Intransitivo & naakat + PARTICÍPIO-verbo-ABSOLUTIVO \\
\hline
\end{tabular}

Nas contruções copulares, a cópula naakat pode ser omitida, como se vê no exemplo em (39) e (40), que são as mesmas sentenças apresentadas em (33) e (34) acima, mas com omissão da cópula (Storto 2010).
Byyty kinda'o-t.
mamão fruta-ABS
'Mamão é fruta'

(40) Taso i-kat-t.

homem PART-dormir-ABS

'O homem dormiu'

Segundo Storto (2010), a cópula poderia ser omitida sempre que estivesse no tempo não futuro. Everett, C. (2006) discorda de Storto (2010), no entanto, e mostra que a cópula não é opcional quando o predicado é um substantivo, conforme ilustra a agramaticalidade da sentença em (41). Os dados em (39) e (41) são contraditórios e revelam a discordância entre os autores.

(41) * Ombaky hĩm.

onça animal

'A onça é um animal'

(exemplo 12.56 de Everett, C. 2006, p. 264) 
Em casos em que há um predicado verbal e a omissão da cópula, como em (40) acima, e (42) abaixo, Everett, C. (2006), diferentemente de Storto (2010), não considera que a sentença possui uma construção de cópula com omissão do verbo copular, mas propõe que essa é uma construção verbal diferente. Para o autor, quando aparece o naakat, a sentenças apresenta uma construção de cópula, e quando o naakat não aparece, mas apenas o verbo intransitivo, ele considera que a sentença possui uma construção que ele denomina construção de valência intransitiva. Essa análise tem consequências para as glosas que autor utiliza. A título de exemplo, apresentamos abaixo duas sentenças copiadas com as glosas do autor. Quando o verbo intransitivo aparece sem a cópula, como em (42), o autor glosa o prefixo $\{i-\}$ como INT de intransitivo e o sufixo $\{-t\}$ como NFUT de não futuro.

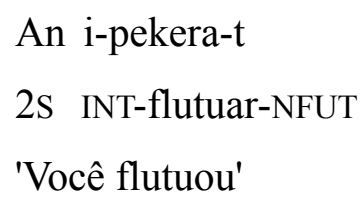

(exemplo 12.12 de Everett, C. 2006, p. 238)

Já em casos em que aparece o verbo copular, como em (43), ele continua glosando o prefixo $\{i-\}$ como INT de intransitivo, mas glosa agora o sufixo do verbo $\{-\varnothing\}$ como COP.AGR. de concordância de cópula. Para o autor, o sufixo verbal $\{-t\}$ ou $\{-\varnothing\}$, embora homófono aos prefixos de não futuro caracterizam uma concordância com com a cópula. Storto (2010) também considera esse sufixo como marcar de concordância, que ela chama de concordância absolutiva.

$$
\begin{aligned}
& \text { I na-aka-t i-kysep- } \varnothing \text {. } \\
& 3 \text { NSAP-COP-NFUT INT-pular-COP.AGR } \\
& \text { 'Ele está pulando' }
\end{aligned}
$$

(exemplo 12.18 de Everett, C. 2006, p. 240)

Os principais argumentos para não se considerar esse morfema como marca de tempo, embora ele seja homófono ao sufixo de não futuro são: (i) esse sufixo também aparece nas construções de cópula com predicado nominal, como mostrou a tabela 5; e (ii) quando a sentença com cópula está no futuro, o sufixo de naaka flexiona, mas não o sufixo do 
predicado, como se pode ver no exemplo (44).

\begin{tabular}{|c|c|c|}
\hline Taso & $\varnothing$-na-aka-j & i-kat-t. \\
\hline homem & 3-DECL-COP-FUT & PART-dormir-ABS \\
\hline
\end{tabular}

(exemplo 8 de Storto 2010, p. 2)

Esta seção apresentou uma construção verbal bastante comum em Karitiana, a construção de cópula. Ela nos ajuda a nos familiarizar com muitos dados deste trabalho que aparecem com verbos intransitivos. A próxima seção tratará dos sintagmas nominais na língua.

\section{$1.4 \quad$ Sintagmas Nominais}

Os sintagmas nominais em Karitiana possuem uma característica singular. Eles aparecem sempre nus, e não possuem nenhum material funcional na posição de determinante. O nomes na língua não possuem tampouco marcas de plural/singular ou classificadores (Müller, Storto e Coutinho-Silva 2006). Os substantivos comuns ocorrem livremente nus como argumentos de sentenças (Müller, Storto e Coutinho-Silva 2006, Müller 2009). O exemplo (45) mostra que tanto nomes comuns que denotam conjuntos de indivíduos, como jonso 'mulher', ou que denotam massa, como ese 'água', que se comportam respectivamente como nomes contáveis e massivos nas línguas do mundo, aparecem nus sem qualquer flexão de número ou quantificador nominal.

$$
\text { Jonso Ø-naka-ot-Ø ese. }
$$

mulher 3-DECL-buscar-NFUTágua

'Mulher buscou água'

Os sintagmas nominais na língua possuem denotação neutra para número (Müller, Storto e Coutinho-Silva 2006). A sentença (45) é apropriada em contextos em que há uma ou mais de uma mulher e qualquer quantidade de água.

Segundo os autores, do ponto de vista tipológico, a língua Karitiana estaria mais 
próxima de línguas como o chinês que apresentam ocorrência livre de nominais nus como argumentos e ausência de flexão nominal (Chierchia 1998). No entanto, essas línguas possuem um sistema de classificadores que ocorrem em sintagmas com numerais. Em Karitiana, no entanto, não há classificadores nesse ambiente, como mostra o exemplo (46).

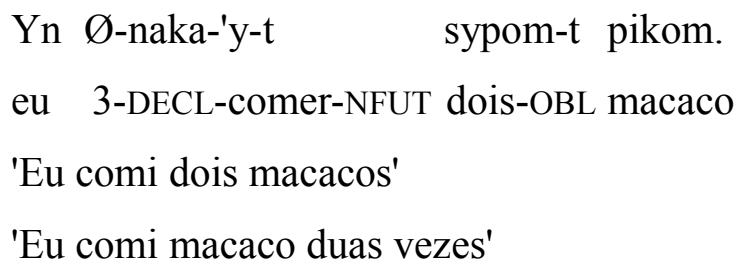

(exemplo 4 de Müller, Storto e Coutinho-Silva 2006, pág. 188)

Segundo Müller (2011), os sintagmas nominais em Karitiana são também neutros quanto à (in)definitude, ou seja, eles não codificam a distinção entre definido e indefinido. Os exemplos abaixo mostram que os sintagmas nominais nus em Karitiana podem aparecer tanto em um contexto de inserção de um novo referente no discurso, ambiente típico de argumentos indefinidos, como em (47a), quanto em contextos de retomada de um referente, contexto típico de argumentos definidos, como em (47b).

$$
\begin{aligned}
& \text { Professor enfermera } \varnothing \text {-na-aka-t } \quad \text { i-amby-t } \quad \text { y-ambip. } \\
& \text { Professor enfermeira } 3 \text {-DECL-COP-NFUT PART-vir-ABS } 1 \text { POSS-casa } \\
& \text { 'Um professor e um enfermeiro vieram a minha casa' }
\end{aligned}
$$

(exemplo 18a de Müller 2011, p. 442)

$$
\begin{aligned}
& \text { b. Professor Ø-na-aka-t i-le-t livro-ty y-'iti hot. } \\
& \text { Professor 3-DECL-COP-NFUT PART-ler-ABS livro-OBL 1POSS-filha para } \\
& \text { 'O professor leu o livro para a minha filha' }
\end{aligned}
$$

(exemplo 18b de Müller 2011, p. 442)

Uma vez que não possui material funcional na posição de determinante, a língua Karitiana possui estratégias para expressar demonstrativos e quantificação universal bem distintas do que se observa em línguas como o inglês e o português. Sentenças com sintagmas interpretados como demonstrativos possuem uma oração relativa (Müller, Storto e Coutinho- 
Silva 2006 e Coustinho-Silva 2008). Para expressar algo como aquele homem, em Karitiana, diz-se homem (que) está lá, como se vê no exemplo em (48).

Dibm Ø-naka-tat-i ony taso aka. amanhã 3-DECL-ir-FUT lá homem COP 'Aquele homem irá amanhã' (['Homem (que) estar lá] irá amanhã')

(exemplo 10 de Müller, Storto e Coutinho-Silva 2006, pág. 189)

E para exprimir a noção de quantificação universal, a língua Karitiana possui uma expressão formada por uma oração subordinada, akatyym, que pode ser traduzida por 'todos(as)' (Coutinho-Silva 2008 e Storto e Thomas 2012).

Taso aka-tyym $\varnothing$-na-sokõ'ĩ-t $\quad$ eremby.
homem COP-ASP DECL-amarrar-NFUT rede
'Todos os homens amarraram rede'

(Storto e Thomas 2012, p. 1)

As características apresentadas acima mostram que, tipologicamente, a língua Karitiana está inserida no grupo de línguas que apresenta apenas quantificação adverbial (Sanchez-Mendes 2009). Partee et el. (1987) e Bach et al. (1995) distinguem dois tipos de quantificação nas línguas naturais: quantificação de determinante e quantificação adverbial. A quantificação de determinante é a quantificação do domínio nominal feita por quantificadores de determinante (como todo e algum em português). A quantificação adverbial engloba todos os outros mecanismos quantificacionais, por meio de advérbios, auxiliares, quantificadores flutuantes, afixos, etc.

Karitiana é uma língua que apresenta apenas quantificação adverbial porque não possui nenhum tipo de quantificador de determinante canônico, embora possua mecanismos de quantificação como akatyym descrito acima, que expressa quantificação universal. Coutinho-Silva (2008) e Storto e Thomas (2012) argumentam que akatyym poderia ser um contraexemplo para a afirmação de que em Karitiana há apenas quantificação adverbial. Segundo os autores, akatyym não possui características de advérbio. Ele quantifica apenas sobre o domínio nominal e não sobre o verbal e possui uma distribuição muito mais rígida em 
relação ao seu domínio de quantificação em comparação com a liberdade de posição dos advérbios.

No entanto, segundo a divisão tipológica proposta em Partee et el. (1987) e Bach et al. (1995), a quantificação adverbial não compreende apenas os advérbios de quantificação, mas todo tipo de quantificação exercida por mecanismos distintos dos quantificadores de determinantes canônicos. Akatyym, embora não se comporte como um advérbio típico em Karitiana, também não é um determinante como o every 'todo' do inglês. Coutinho-Silva (2008) analisa akatyym como uma sentença relativa, enquanto Storto e Thomas (2012) defendem que se trata de uma sentença adverbial. Logo, continuaremos considerando que o Karitiana é uma língua que não possui quantificadores nominais que são determinantes, mesmo possuindo construções que fornecem significados semelhantes aos dos quantificadores nominais, como o caso de akatyym.

Um outro possível contraexemplo para a assunção de que em Karitiana não há quantificação nominal seria os numerais. No entanto, os numerais têm comportamento de adjunto e não ocupam posição funcional de determinante na língua. O exemplo (46), repetido aqui em (50) mostra que o numeral sypom 'dois' pode ser considerado ora como adjunto do nome, ora como adjunto adverbial da sentença.

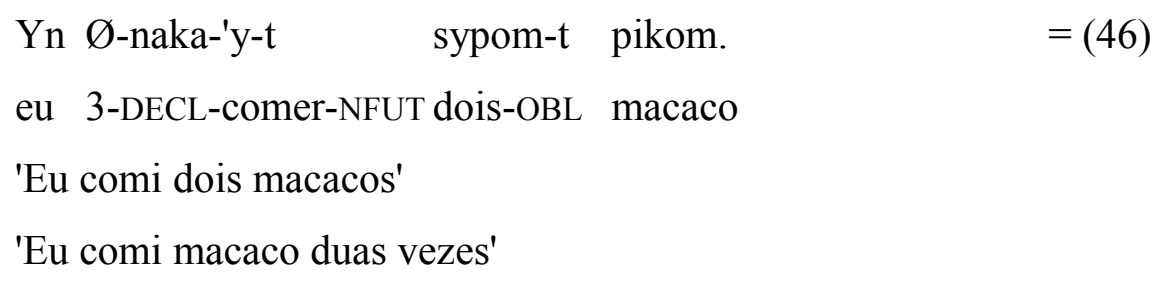

(exemplo 4 de Müller, Storto e Coutinho-Silva 2006, pág. 188)

Esta subseção mostrou que a língua Karitiana não possui material funcional na posição de determinante e se encaixa no grupo de línguas que possuem apenas quantificação adverbial (Sanchez-Mendes 2009). Esta subseção fecha a seção sobre as características básicas da morfossintaxe do Karitiana. O objetivo principal foi ajudar a deixar mais claros os exemplos deste trabalho para aqueles que nunca tiveram nenhum contato com a língua. A próxima seção faz uma caracterização do paradigma teórico utilizado neste trabalho. Seu objetivo é tornar este trabalho acessível para leitores interessados nesta pesquisa que ainda não estejam familiarizados com os mecanismos da Semântica Formal. 
O objetivo desta seção é descrever de modo sucinto o modelo teórico da Semântica Formal adotado neste trabalho de modo a deixar claro quais são as perguntas que o norteiam. O leitor que estiver familiarizado com a teoria pode desconsiderar esta seção. A descrição apresentada foi formulada basicamente a partir de dois livros introdutórios: Semantics in Generative Grammar de Irene Heim e Angelika Kratzer (Heim e Kratzer 1998) e Informal Lectures in Formal Semantics de Emmon Bach (Bach 1989). A subseção sobre eventos foi baseada em textos clássicos sobre o assunto, como Davidson (1967), Parsons (1990) e Kratzer (1996, 2003).

A Semântica, em seu sentido geral, é o estudo do significado. Diferentes modos de descrever o que é significado moldam diferentes tipos de semânticas. O modelo teórico adotado nesta pesquisa é o da Semântica Formal (doravante SF) e o seu objeto linguístico de interesse é a sentença. A SF não busca entender aspectos do significado de uma palavra específica, como a Semântica Lexical, nem o significado de unidades maiores que a sentença, como a Semântica Discursiva ${ }^{13}$.

A Semântica Formal descreve o significado das sentenças das línguas naturais como as suas condições de verdade. Saber o significado de uma sentença é saber quais são as condições necessárias para que essa sentença seja verdadeira. Não é relevante, para a análise, o valor de verdade de uma sentença, ou seja, saber se ela é verdadeira ou falsa, mas saber em que condições a sentença é considerada verdadeira. É nesse sentido que se diz que a Semântica Formal é uma teoria verifuncional (de truth-conditional semantics). Assim, a SF é uma teoria do significado que emparelha sentenças com suas condições de verdade em um esquema como (51), abaixo:

A sentença “__ " é verdadeira se, e somente se,

$\mathrm{Na}$ fórmula em (51), o que aparece entre aspas, no primeiro espaço, é a sentença da língua objeto, a língua que está sendo investigada. Neste trabalho, a língua objeto é o Karitiana. No segundo espaço, depois de se, e somente se, aparece a descrição das condições de verdade da sentença. Essa descrição é feita por meio de uma metalinguagem, que é a

\footnotetext{
13 Semântica Lexical, Semântica Formal e Semântica Discursiva não esgotam os tipos de semânticas existentes. Ver Pires de Oliveira (1999) para uma descrição mais completa dos estudos do significado.
} 
língua utilizada para se falar da língua objeto. A metalinguagem utilizada pela teoria poderia ser qualquer uma, como o inglês, o português, ou o Karitiana. No entanto, a fim de explicitar fenômenos semânticos bastante precisos, a SF utiliza uma metalinguagem enriquecida com um vocabulário técnico nas suas representações. Desse modo, a metalinguagem utilizada nesse trabalho será a língua portuguesa acrescida de um vocabulário técnico, que virá sempre acompanhado de uma explicação ou tradução informal.

O objetivo final de uma análise semântica é explicitar, por meio de uma forma lógica elaborada na metalinguagem, as condições de verdade, e portanto, o significado de uma sentença de uma língua natural ${ }^{14}$. Esse princípio é baseado na proposta de Richard Montague (Montague 1970, 1973), que é uma das ideias centrais na origem da Semântica Formal, de que as línguas naturais poderiam ser tratadas como linguagens formais.

Mas esse é apenas o resultado da análise. O trabalho do linguista está em analisar como o sistema semântico produziu esse significado. Para isso, ele precisa entender como o encadeamento de palavras que forma a sentença pode ser computado para gerar as suas condições de verdade. É nesse sentido que se assume que a interpretação semântica é composicional. O significado de uma sentença depende do significado de suas partes e de sua estrutura sintática, ou seja, de como as suas partes estão organizadas. Assim, a SF procura investigar de que modo o significado de cada parte de uma sentença contribui para as suas condições de verdade.

A composição semântica que permite computar o significado de uma sentença consiste em dois componentes básicos. Um léxico e um conjunto de regras. O léxico representa as expressões linguísticas acompanhadas de seu significado, ou seja, sua denotação. As regras, por sua vez, descrevem como essas expressões podem ser associadas ${ }^{15}$.

A denotação de cada item lexical é representada por dois colchetes duplos 【 \. Assim, para se referir à denotação de um nome comum como cadeira, utiliza-se 【 cadeira 】. Não está no escopo desta seção fazer uma descrição completa das denotações de todos os tipos de itens linguísticos. Vamos nos ater somente em algumas categorias para explicar a relação semântica básica de Aplicação Funcional.

Uma das formas de descrever a composição semântica é através da terminologia

\footnotetext{
14 A forma lógica é entendida aqui enquanto uma representação semântica da sentença e não como nível de representação sintática. Ver Kratzer (1996) para uma discussão sobre a distinção entre os conceitos.

15 A descrição nesta seção está bastante simplificada. Não foram inseridas, por exemplo, as descrições dos domínios de denotação dos itens lexicais. Para mais detalhes sobre o instrumental teórico da Semântica Formal ver Heim e Kratzer (1998).
} 
utilizada por Gottlob Frege, um lógico, filósofo e matemático alemão que teve grande influência na lógica e na filosofia da linguagem, disciplinas que estão na origem da Semântica Formal. Segundo Frege (1892), os constituintes de uma sentença podem ser saturados ou insaturados. Os constituintes saturados possuem significado completo e podem funcionar como argumentos nas sentenças. São constituintes saturados, por exemplo, os nomes próprios, as descrições definidas e as sentenças. Os constituintes insaturados são partes incompletas que necessitam de um ou mais complementos para se tornarem saturadas, ou seja, possuírem valor de verdade; elas são entendidas como funções que necessitam de argumentos. São insaturados, por exemplo, os verbos antes de se associarem a seus argumentos.

$\mathrm{Na}$ proposta de Frege, as sentenças das línguas naturais são itens saturados e possuem como denotação o seu valor de verdade ${ }^{16}$. Convencionou-se utilizar o número 1 para o valor verdadeiro e 0 para o falso. A denotação de um nome próprio como Inácio é saturada e equivale a sua referência, o próprio indivíduo Inácio. Sua descrição é feita da seguinte forma:

\section{【Inácio 》= Inácio}

O que está apresentado em (52) é a entrada lexical do item Inácio para a computação semântica. Ela pode ser lida da seguinte forma: a denotação de Inácio é o indivíduo Inácio. A denotação de um verbo intransitivo como trabalha é insaturada e é representada por uma função. O formalismo mais recente da SF trabalha com uma notação especial para descrever funções: a notação- $\lambda$ (lambda). Trata-se apenas de uma forma de descrição das funções. Assim, uma função matemática como em (53a) pode ser reescrita com a notação lambda como em (53b).
a. $\quad f(x)=x+1$
b. $\quad \lambda x \cdot x+1$

A descrição em (53b) representa uma função insaturada que precisa de um argumento. A parte que representa que com um argumento a função será saturada é exatamente o $\lambda \mathbf{x}$. Uma função como trabalha tem a seguinte entrada lexical em notação- $\lambda$ :

\footnotetext{
${ }_{16}$ A denotação não é o mesmo que significado. Uma sentença denota um valor de verdade (verdadeiro ou falso), mas seu significado é descrito por meio de condições de verdade, não propriamente em valores de verdade.
} 
A descrição em (54) deve ser lida da seguinte forma: trabalha é uma função que pega um argumento $\mathbf{x}$ e devolve um item saturado do tipo sentença (trabalha $(x)$ ). Mais especificamente, devolve o valor de verdade 1 se o valor atribuído a $\mathbf{x}$ trabalha e 0 se o valor dado a $\mathbf{x}$ não trabalha. Uma forma pela qual se pode saturar a função trabalha é por meio de uma saturação com nome próprio, do tipo Inácio trabalha ${ }^{18}$. Nesse caso, aplica-se a função

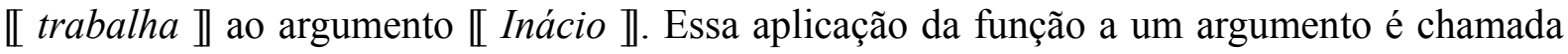
de Aplicação Funcional e é escrita da seguinte forma:

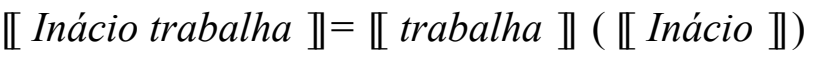

Substituindo os itens por sua denotação:

【Inácio trabalha $\rrbracket=[\lambda \mathrm{x}$. trabalha $(\mathrm{x})]$ (Inácio)

$\mathrm{O}$ argumento Inácio vai saturar a função por meio de $\beta$-redução (beta-redução). $\beta$ redução é a redução do termo- $\lambda$, ou seja, é a substituição do termo- $\lambda$ pelo argumento. Como a função da denotação de trabalha tem uma insaturação, ou seja, necessita de um argumento, ela tem um lambda. Esse lambda desaparece juntamente com a variável com a Aplicação Funcional, o argumento Inácio substitui todas as ocorrências da variável $\mathbf{x}$ e a função é reduzida. O resultado da aplicação é a denotação da sentença, ou seja um valor de verdade (Frege 1892). A sentença terá o valor de verdade 1, o verdadeiro, se Inácio trabalha, e o valor de verdade 0 , o falso, nos outros casos. A relação entre o valor de verdade de uma sentença (sua denotação) e as sua condições de verdade (seu significado) são representados da seguinte forma:

17 Esta é apenas uma simplificação uma vez que a condição de domínio das funções não está sendo levada em conta. As informações sobre o tempo estão sendo descartadas por questão de simplificação.

18 Há outras regras de composição semântica disponíveis no modelo. A regra de modificação, por exemplo, será descrita e utilizada no capítulo desta tese. Ver Heim e Kratzer (1998) para mais detalhes. 
Ou, ainda:

$$
\text { "Inácio trabalha" é verdadeira se, e somente, se trabalha (Inácio) }
$$

O resultado que se vê em (57) e (58), embora possa parecer à primeira vista apenas uma tradução banal, não é nada trivial. Vimos que o que aparece entre colchetes duplos em (57) e entre aspas em (58) é uma expressão linguística, nesse caso, a sentença Inácio trabalha na língua objeto. Os colchetes duplos representam uma função de interpretação que aplicada à sentença fornece a sua denotação, que é descrita depois do sinal de igual. A denotação da sentença é descrita por meio de uma condição, expressa depois de se, e somente se. Esta condição é fornecida por meio de uma metalinguagem. Se a sentença analisada estivesse em Karitiana, a representação seria a seguinte:
a. 【Inácio ipytim'adnat $\rrbracket=1$ se, e somente, se trabalha (Inácio)
b. "Inácio ipytim'adnat" é verdadeira se, e somente, se trabalha (Inácio)

Em (59), a língua objeto é o Karitiana e a metalinguagem descrita em língua portuguesa acrescida de uma linguagem técnica, já que a predicação é descrita como trabalha (Inácio), representação inspirada na Lógica de Predicados. Vimos, portanto, um exemplo de composição semântica de expressões linguísticas que resultou em um pareamento entre uma sentença e seu significado descrito por meio de uma condição de verdade, escrita em uma metalinguagem.

Essa é a descrição básica do que é a predicação no modelo da SF. No caso descrito acima, a Aplicação Funcional envolveu um nome próprio e um verbo intransitivo. No entanto, esse não é único ambiente que a Aplicação Funcional pode ocorrer. Na realidade, a composição semântica é indiferente aos rótulos das categorias sintáticas. A Aplicação Funcional pode ocorrer sempre que houver uma compatibilidade entre os termos. Essa compatibilidade é definida por meio de tipos semânticos. É nesse sentido que se diz que

19 Heim e Kratzer (1998) utilizam o próprio inglês como metalinguagem. Estamos usando uma representação inspirada na Lógica de Predicados. Na Lógica de Predicados, os predicados são representados por letras maiúsculas e os argumentos por letras minúsculas. Desse modo a predicação Inácio trabalha seria representada como $T(i)$. Estamos colocando a representação em palavras por questões de simplificação. 
interpretação semântica é conduzida por meio de tipos (do inglês type-driven interpretation).

Os dois tipos básicos assumidos pela SF são: tipo e para indivíduos, como Inácio; e tipo t para valores de verdade, o tipo das sentenças. Esses são os tipos correspondentes às denotações saturadas. Os tipos das denotações insaturadas são formados a partir desses dois tipos básicos. Um verbo intransitivo como trabalha, por exemplo, tem um tipo composto. Seu tipo é <e,t>. Isso quer dizer que trabalha é uma função que pede um argumento de tipo e, tipo dos indivíduos, e devolve um constituinte do tipo t, ou seja, uma sentença. As denotações em (60) mostram os tipos de cada item utilizado na composição descrita acima.

$$
\begin{array}{ll}
\text { a. } & {[[\text { Inácio }]]_{\mathrm{e}}=\text { Inácio }} \\
\text { b. } & {[[\text { trabalha }]]_{<\mathrm{e}, \triangleright}=\lambda \mathrm{x}_{\mathrm{e}} \text {. trabalha }(\mathrm{x})} \\
\text { c. } & {\left[[\text { Inácio trabalha }]_{\mathrm{t}}=1 \mathrm{se}, \mathrm{e}\right. \text { somente, se trabalha (Inácio) }}
\end{array}
$$

Um verbo transitivo como amar possui duas insaturações, ou seja, precisa de dois argumentos do tipo dos indivíduos para formar uma sentença. O tipo de um verbo como esse é portanto, $<\mathrm{e},<\mathrm{e}, \mathrm{t}>>$. Isso quer dizer que ele é uma função que pega um argumento do tipo e, o tipo dos indivíduos, e devolver uma função do tipo <e,t>. Essa segunda função, por sua vez, pega um argumento do tipo e e devolve uma sentença, de tipo t. A composição semântica da João ama Maria está descrita abaixo:

$$
\begin{array}{ll}
\text { a. } & {\left[\left[\text { João } \rrbracket_{\mathrm{e}}=\right.\right.\text { João }} \\
\text { b. } & {[[\text { Maria }]]_{\mathrm{e}}=\text { Maria }} \\
\text { c. } & {[[\text { ama }]]_{<\mathrm{e},<\mathrm{e}, \triangleright}=\lambda \mathrm{x}_{\mathrm{e}} \lambda \mathrm{y}_{\mathrm{e}} \text {. ama }(\mathrm{x})(\mathrm{y})} \\
\text { d. } & {\left[\left[\text { ama Maria } \rrbracket_{<\mathrm{e}, \triangleright}=\lambda \mathrm{y}_{\mathrm{e}} \text { ama }(\text { Maria })(\mathrm{y})\right.\right.} \\
\text { e. } & {[[\text { João ama Maria }]]_{\mathrm{t}}=1 \text { se, e somente, se ama (Maria)(João) }{ }^{20}}
\end{array}
$$

Uma vez descrito o instrumental teórico básico utilizado neste trabalho, a próxima seção avançará na descrição da SF que considera eventos em sua teoria.

20 Embora a ordem ama(Maria)(João) possa parecer incorreta, ela quer dizer que João ama Maria. Isso ocorre porque os argumentos são representados na ordem em que são inseridos na sentença. Como o primeiro argumento a saturar a sentença é o objeto, ou seja, o argumento interno, ele ficará sempre representado mais próximo ao verbo. Essa representação está sendo utilizada porque é a mais comum nos trabalhos em Semântica Formal atuais. Na Lógica de Predicados Clássica, a representaria seria A(j,m). 


\subsection{Semântica de Eventos}

A seção anterior mostrou que a denotação de um verbo intransitivo é uma função do tipo $<$ e, $\mathrm{t}>$ que toma um argumento do tipo e e devolve uma sentença. Trabalhos recentes em SF têm procurado revisar essa denotação tradicional com argumentos de que os verbos não podem ser considerados apenas funções de indivíduos e devem denotar eventos. Esta seção pretende introduzir a noção de eventos no modelo semântico descrito acima.

O filósofo Donald Davidson foi o primeiro a discutir a importância da noção de eventos em uma teoria do significado. Davidson (1967) defende que os predicados verbais além dos seus argumentos usuais possuem também uma variável de evento.

a. Denotação tradicional:

$[[$ ferir $]]=\lambda \mathrm{x} . \lambda \mathrm{y}$. ferir $(\mathrm{x})(\mathrm{y})$

b. Denotação davidsoniana:

$[[$ ferir $]]=\lambda \mathrm{x} . \lambda \mathrm{y} . \lambda \mathrm{e} . \operatorname{ferir}(\mathrm{x})(\mathrm{y})(\mathrm{e})$

Parsons (1990), por sua vez, apresenta uma série de argumentos em favor de verbos denotarem eventos mostrando os ganhos empíricos na descrição semântica. No entanto, a proposta de Parsons (1990) é ligeiramente diferente da de Davidson (1967). Davidson (1967) considera que o evento é mais um argumento do verbo, enquanto que Parsons (1990) considera que os verbos são predicados de eventos, ou seja, os eventos são os únicos argumentos dos verbos, e seus argumentos nominais são inseridos na forma lógica via predicação secundária, representada por seus papéis temáticos.

a. $\quad$ Proposta de Davidson (1967):

$[[$ ferir $]=\lambda \mathrm{x} . \lambda \mathrm{y} . \lambda \mathrm{e}$. ferir $(\mathrm{x})(\mathrm{y})(\mathrm{e})$

b. Proposta de Parsons (1990):

[[ ferir $\rrbracket=\lambda \mathrm{x} . \lambda \mathrm{y} . \lambda \mathrm{e}$. ferir (e) \& Agente (y)(e) \& Tema (x)(e)

Um dos ganhos dessa proposta diz respeito ao uso de modificadores verbais em sentenças como (64) abaixo. 
Vimos que o tratamento semântico dos verbos baseado na tradição lógica os considera como relações entre os seus argumentos. O verbo ferir, por exemplo, denota uma relação entre dois argumentos, aquele que fere e aquele que é ferido. A sentença Brutus feriu César é representada tradicionalmente como em (65).

【Brutus feriu César $\rrbracket=1$ se, e somente, se feriu (César)(Brutus)

Nessa abordagem, uma sentença modificada como (66) tem a seguinte representação.

$$
\text { 【 Brutus feriu César com uma faca 》= } 1 \text { sse feriu (César)(Brutus)(com uma faca) }
$$

Essa não é uma forma totalmente adequada de representar as sentenças modificadas por duas razões. Primeiramente, essa representação leva à pressuposição de que o verbo ferir possuiria entradas lexicais diversas com um número diferente de posições insaturadas. $\mathrm{Ou}$ seja, se ele vier sem modificador ele será uma relação com duas posições insaturadas, se ele tiver um modificador, possuirá três insaturações, se tiver dois modificadores, quatro insaturações e assim por diante. Não é interessante para a teoria inchar o léxico com tantas entradas para o mesmo verbo.

A segunda razão é a perda da preservação de relações semânticas importantes entre as sentenças modificadas, como o acarretamento. Os exemplos em (67) mostram que as representações não conseguem preservar o acarretamento existente entre as sentenças. A representação em (67a') não acarreta as representações em (67b') e (67c'), mas a sentença (67a) acarreta as sentenças (67b) e (67c). As representações em (67a'), (67b'), e (67c') não acarretam a representação em (67d'), mas as sentenças (67a), (67b) e (67c) acarretam a sentença (67d). 
a. Brutus feriu César com uma faca nas costas.

a'. $\quad[[$ Brutus feriu César com uma faca nas costas $]]=1$ sse feriu (César) (Brutus)(com uma faca)(nas costas)

b. Brutus feriu César com uma faca.

b'. $\quad[[$ Brutus feriu César com uma faca $\rrbracket=1$ sse feriu (César)(Brutus)(com uma faca)

c. Brutus feriu César nas costas.

c'. $\quad[[$ Brutus feriu César nas costas ]] = 1 sse feriu (César)(Brutus)(nas costas)

d. Brutus feriu César.

d'. $\quad[[$ Brutus feriu César $\rrbracket=1$ sse feriu (César)(Brutus)

Uma representação semântica com eventos e a separação dos argumentos dos verbos por meio dos papéis temáticos resolve o problema apresentado acima. Na proposta de Parsons (1990), a sentença sem modificadores como (68a), tem uma representação semântica como em (68b).
a. Brutus feriu César.
b. $\quad[[$ Brutus feriu César $\rrbracket=1$ sse $\exists$ e $[$ ferir (e) \& Agente (Brutus)(e) \& Tema (César)(e) $]^{21}$

A representação em (68b) pode ser lida da seguinte forma: existe um evento, esse é um evento de ferir, o agente desse evento é Brutus e o tema desse evento é César. Com representações desse tipo, é possível preservar os acarretamentos discutidos nas sentenças em (69).

(69) a. Brutus feriu César com uma faca nas costas.

a'. $\quad[[$ Brutus feriu César com uma faca nas costas $]=1$ sse $\exists$ e [ ferir (e) \& Agente (Brutus)(e) \& Tema (César)(e) \& Com (faca)(e) \& Em (costas)(e) ]

b. Brutus feriu César com uma faca.

b'. [[ Brutus feriu César com uma faca $]=1$ sse $\exists$ e $[$ ferir (e) \& Agente (Brutus)(e) \& Tema (César)(e) \& Com (faca)(e) ]

\footnotetext{
21 A variável evento é presa existencialmente por um quantificador existencial sentencial.
} 
c. Brutus feriu César nas costas.

c'. $\quad[[$ Brutus feriu César nas costas ]] $=1$ sse $\exists$ e [ ferir (e) \& Agente (Brutus)(e) \& Tema $($ César $)(\mathrm{e}) \&$ Em $(\operatorname{costas})(\mathrm{e})]$

d. Brutus feriu César.

d'. $\quad[[$ Brutus feriu César ] ] $=1$ sse $\exists \mathrm{e}[$ ferir (e) \& Agente (Brutus)(e) \& Tema (César)(e) ]

Pode-se observar em (69) que, com uma variável de eventos (e), os modificadores aparecem na representação semântica por meio da conjunção \&. Uma vez que uma sentença complexa do tipo $P \& Q$ só é verdadeira se $P$ for verdadeira e $Q$ for verdadeira, o acarretamento das sentenças é mantido.

\section{Tabela 6: Tabela de Verdade da Conjunção}

\begin{tabular}{|c|c|c|}
\hline P & Q & P\&Q \\
\hline V & V & V \\
\hline V & F & F \\
\hline F & V & F \\
\hline F & F & F \\
\hline
\end{tabular}

Um outro argumento apresentado por Parsons (1990) em favor de uma teoria que considere eventos é o fato de que algumas sentenças das línguas naturais fazem referência explícita a eventos. Por exemplo, em inglês o gerúndio nominal singing do verbo to sing 'cantar' faz referência explícita a um evento.

(70) After the singing of the Marseillaise they saluted the flag.

'Depois do canto da Marselhesa, eles saudaram a bandeira'

(exemplo de Parsons 1990, p. 17)

A Semântica de Eventos captura adequadamente o fato de que o gerúndio nominal em inglês se refere a um evento, embora seja uma categoria nominal. A representação semântica da sentença acima é a seguinte: 
(71) $\exists$ e [ saudar (e) \& Agente (eles)(e) \& Tema (a bandeira)(e) \& ヨe' [ cantar (e') \& Tema(a Mars.)(e') \& Depois (e)(e') ] ]

Além de as línguas naturais fazerem referência explícita a eventos, elas apresentam também a capacidade de fazer quantificações sobre eventos. É o que mostra a sequência de sentenças em (72).

(72) a. Em todo incêndio, oxigênio é consumido.

b. Agatha incendiou a casa.

c. Oxigênio foi consumido.

(traduzido do exemplo de Parsons 1990, p. 18)

Uma representação semântica com eventos consegue explicar porque (72c) é uma consequência lógica de (72a) e (72b). A quantificação universal explícita sobre eventos em (72) é semelhante à encontrada no famoso silogismo aristotélico descrito em (73).

(73) a. Todo homem é mortal.

a'. $\quad[[$ Todo homem é mortal $\rrbracket=1$ sse $\forall \mathrm{x}[\operatorname{homem}(\mathrm{x}) \rightarrow \operatorname{mortal}(\mathrm{x})]$

b. Sócrates é homem.

b'. [[ Sócrates é homem $]=1$ sse homem(Sócrates)

c. Sócrates é mortal.

c'. $\quad[[$ Sócrates é mortal $\rrbracket=1$ sse mortal(Sócrates)

A inferência em (72) pode então ser escrita da seguinte forma:

(74) a. [[ Em todo incêndio, oxigênio é consumido ]] $=1$ sse $\forall \mathrm{e}[$ incendiar (e) $\rightarrow$ $\exists \mathrm{e}^{\prime}\left[\operatorname{consumir}\left(\mathrm{e}^{\prime}\right) \& \mathrm{Tema}\left(\mathrm{O}_{2}\right)\left(\mathrm{e}^{\prime}\right) \& \operatorname{Em}\left(\mathrm{e}^{\prime}\right)(\mathrm{e})\right]$ ]

b. $\quad[[$ Agatha incendiou a casa $]=1$ sse $\exists \mathrm{e}[$ incendiar (e) \& Agente (Agatha) (e) \& Tema (a casa)(e) ]

c. $\quad\left[\left[\right.\right.$ Oxigênio foi consumido $\rrbracket=1 \mathrm{sse} \exists \mathrm{e}^{\prime}\left[\operatorname{consumir}(\mathrm{e}) \& \operatorname{Tema}\left(\mathrm{O}_{2}\right)\left(\mathrm{e}^{\prime}\right)\right]$ 
Nota-se, portanto, que a SF tem um ganho descritivo com o uso da noção de eventos. O conjunto de denotações básicas com indivíduos e valores de verdade descrito acima será, então, enriquecido com os predicados de eventos. Além dos dois tipos básicos e para indivíduos e $\mathbf{t}$ para valores de verdade, um tipo $\mathbf{s}$ é inserido para os eventos. Um verbo intransitivo que foi descrito anteriormente como uma função do tipo <e,t> que toma um argumento do tipo e e devolve uma sentença, de tipo t, passa a ser considerado como uma função do tipo $<$ e, $<$ s, $\mathrm{t}>>$ na proposta de Parsons (1990), chamada pela literatura de proposta neodavidsoniana. Um verbo transitivo que tinha tipo $<\mathrm{e},<\mathrm{e}, \mathrm{t}>>$ na teoria tradicional passa a ter o tipo $<\mathrm{e},<\mathrm{e},<\mathrm{s}, \mathrm{t}>>>$ nessa nova proposta.

Há um desenvolvimento mais recente dessa proposta que diz respeito à implementação de uma ideia já bastante difundida nos estudos da teoria da gramática de que o argumento externo de um verbo tem um estatuto diferente do argumento interno. Kratzer (1996) retoma os argumentos de Marantz (1984) de que o argumento externo não é um verdadeiro argumento do verbo. O primeiro deles é que muitas vezes um tipo particular de argumento interno desencadeia uma interpretação particular nem sempre prevista pela raiz verbal. Em inglês, por exemplo take a bus 'tomar/pegar um ônibus' e take a nap 'tirar um cochilo' têm interpretações muito distintas, embora sejam sintagmas verbais formados pelo mesmo verbo take. O mesmo vale para matar uma barata e matar uma garrafa de cerveja em português. O mesmo fenômeno não ocorre com argumentos externos.

Essa característica (entre outras) levou os estudiosos a propor que o argumento interno tem uma relação com a denotação do verbo diferente da do argumento externo. Mais especificamente, motivou a considerar que o argumento interno é o único verdadeiro argumento do verbo. Assumindo essa proposta, Kratzer (1996) propõe que a denotação de um verbo transitivo seja a seguinte:

$$
[\text { ferir }]]=\lambda \mathrm{x} . \lambda \mathrm{e} . \text { ferir }(\mathrm{x})(\mathrm{e})
$$

A proposta da autora é de que todos os argumentos externos são associados por método neodavidsoniano. O método neodavidsoniano refere-se a uma proposta como a de Parsons (1990) de inserção dos argumentos via predicação secundária. A diferença é que na proposta de Kratzer (1996) a predicação secundária não aparece na denotação do verbo, mas é introduzida sintaticamente por meio de um núcleo funcional, que a autora chama de VoiceP. 
Isso quer dizer que no nível do VP o verbo encontrará apenas o seu argumento interno. A composição semântica de feriu César, por exemplo, fica da seguinte forma:
a. $\quad[[$ feriu $\rrbracket=\lambda x . \lambda e .[$ feriu $(\mathrm{x})(\mathrm{e})]$
b. $\quad[[$ feriu César $\rrbracket=\lambda e .[$ feriu $($ César $)(\mathrm{e})]$

Nesse ponto, há um sintagma verbal (VP) que possui apenas a insaturação do evento e não possui uma insaturação para indivíduos, para que o sujeito possa entrar na derivação. Nesse ponto fica claro que, na proposta de Kratzer (1996), o sujeito não é um verdadeiro argumento do verbo, uma vez que os verdadeiros argumentos do verbo já foram saturados.

A autora propõe que o argumento externo seja inserido sintaticamente por voz, no núcleo de VoiceP, que tem a seguinte denotação:

$$
\text { 【Vice } \left.\rrbracket_{<e,<\mathrm{s}, \triangleright>}=\lambda \mathrm{x}_{\mathrm{e}} \lambda \mathrm{e}_{\mathrm{s}} \text {. [ agente }(\mathrm{x})(\mathrm{e})\right]
$$

O núcleo Voice se junta ao VP por meio de uma nova regra composicional chamada Identificação do Evento (descrita em Kratzer 1996). Trata-se de uma regra de conjunção que pega como input uma função $\mathrm{f}$ do tipo $<\mathrm{e},<\mathrm{s}, \mathrm{t}>>$ (o núcleo Voice) e uma função $\mathrm{g}$ do tipo $<$,,$>$ (o VP de Kratzer) e devolve como output uma função h do tipo $<$ e, $<$ s, $\mathrm{t}>>$ que é representada por $\lambda \mathrm{x}_{\mathrm{e}} \lambda \mathrm{e}_{\mathrm{s}}$. [ $\left.\mathrm{f}(\mathrm{x})(\mathrm{e}) \& \mathrm{~g}(\mathrm{e})\right]$. A conjunção do dois gera uma função $\mathrm{h}$ que é $\lambda \mathrm{x}_{\mathrm{e}}$ $\lambda \mathrm{e}_{\mathrm{s} .} .[[[$ voice $]](\mathrm{x})(\mathrm{e}) \&[[\mathrm{VP}]](\mathrm{e})]$.

$$
\begin{aligned}
& \text { Identificação do Evento: } \\
& \begin{array}{lcl}
\mathrm{f} & \mathrm{g} & \mathrm{h} \\
<\mathrm{e},<\mathrm{s}, \mathrm{t}>> & <\mathrm{s}, \mathrm{t}> & <\mathrm{e},<\mathrm{s}, \mathrm{t}>>
\end{array}
\end{aligned}
$$

No caso da sentença Brutus feriu César, o núcleo Voice se junta ao VP feriu César formando: 
(79)
a. $\quad[[$ Voice $\rrbracket(\llbracket$ feriu César $\rrbracket)=\lambda x . \lambda$ e. [ [ voice ]] $(\mathrm{x})(\mathrm{e}) \&[[\mathrm{VP}]](\mathrm{e})]$

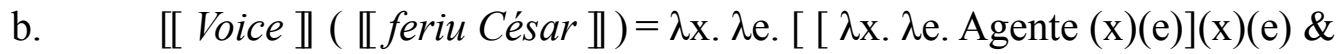
[ $\lambda$ e. feriu (César)(e) ](e) ] ]

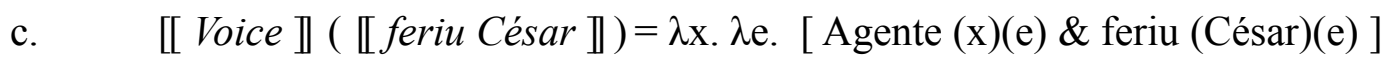

Nesse ponto, é possível inserir o sujeito Brutus por Aplicação Funcional:

(80) a. $\quad[[$ Brutus feriu César $]]=[\llbracket$ Voice $\rrbracket(\llbracket$ feriu César $\rrbracket)]([[$ Brutus $\rrbracket)$

b. $\quad[[$ Brutus feriu César $\rrbracket=[\lambda x$. $\lambda$ e. [ Agente (x)(e) \& feriu (César)(e) ] ] (Brutus)

c. $\quad[[$ Brutus feriu César $]]=\lambda$ e. $[$ Agente (Brutus)(e) \& matou $($ César)(e) ]

A variável evento é presa existencialmente por um quantificador existencial sentencial:

$$
\text { [I Brutus feriu César } \rrbracket_{\mathrm{t}}=1 \text { sse } \exists \text { e [ agente (Brutus)(e) \& feriu(César)(e) ] }
$$

A expressão apresentada acima pode ser lida da seguinte forma: a sentença Brutus feriu César tem denotação 1, ou seja, é verdadeira, se, e somente se, existe um evento, Brutus é o agente desse evento, e esse é um evento de ferir César. Por questões de simplicidade, todas as informações de tempo e aspecto estão sendo desconsideradas. Esta subseção fecha a seção sobre o paradigma teórico da Semântica Formal. O objetivo principal da seção foi o de familiarizar o leitor com o instrumental teórico empregado nesta tese. 


\section{Capítulo 2}

\section{Metodologia}

\section{Introdução}

O objetivo deste capítulo é descrever a metodologia utilizada nos trabalhos de campo para coleta de dados do Karitiana para esta pesquisa, esclarecendo algumas questões teóricas, práticas e éticas. A primeira seção tem como objetivo mostrar de que modo a escolha do quadro teórico de uma pesquisa determina a metodologia utilizada para coletar os seus dados. Além disso, este capítulo também justifica o modelo utilizado. Embora haja um considerável número de trabalhos publicados dedicados à pesquisa de campo, esse ainda é um assunto que suscita debate e discordância entre linguistas indigenistas ${ }^{22}$. A primeira subseção da seção discute mais amplamente o que é metodologia de trabalho de campo em linguística. Muitas afirmações são feitas de forma genérica. A subseção 1.2, no entanto, apresenta concretamente de que modo essas questões tomam forma quando se trata de um caso específico, como o dos quantificadores adverbiais da língua Karitiana com o paradigma teórico da Semântica Formal. Alguns exemplos serão discutidos, a fim de deixar claro de que modo os trabalhos de campo para coleta de dados para esta pesquisa foram feitos. A seção 1.3 discute, por fim algumas questões éticas envolvidas em um trabalho como este.

A segunda seção do capítulo apresenta uma discussão acerca da interface entre a Semântica e a Pragmática na análise da quantificação de frequência em Karitiana. Essa pesquisa mostra de que modo um estudo que procura dividir certos componentes do significado gramatical de uma língua necessita de uma metodologia específica e precisa. $\mathrm{O}$ trabalho sugere alguns ajustes no protocolo que vem sendo utilizado para trabalhos de campo em Semântica Formal.

22 Ver, por exemplo, Crowley (2007), Newman and Ratliff (2001), e Chelliah e de Reuse (2011). 
O trabalho de campo em Linguística, em seu sentido tradicional, envolveu, por muito tempo, um pesquisador vivendo na comunidade que falava a língua que se pretendia estudar. Pelo método de imersão, o linguista teria acesso à língua em seu contexto natural. Esse método foi o predominante desde as primeiros estudos das línguas indígenas americanas feitos por missionários no século XVI até o apogeu da tradição estruturalista americana no século XX.

Linguistas como Sapir e Bloomfield seguiram a tradição estruturalista de trabalho de campo na qual a coleta de dados não se distinguia da interação e observação direta das comunidades estudadas. Nessa tradição, toda a linguística descritiva tinha origem em trabalhos de campo com interação com falantes nativos (Chelliah e de Reuse 2011). Esse é o modo de trabalho de campo reconhecido como legítimo e defendido por muitos linguistas como Everett, D. (2001) e Aikhenvald (2007). Bowern (2008), por exemplo, define o trabalho de campo como somente aquele em que uma língua é estudada no lugar onde ela é falada pelos seus falantes.

Outros linguistas pós-estruturalistas reconhecem, no entanto, que é possível fazer trabalhos de campo com bastante sucesso por meio de coletas baseadas em entrevistas, sem a necessidade de o linguista se tornar um membro da comunidade que fala a língua estudada. Segundo Chelliah e de Reuse (2011), não há uma diferença qualitativa da coleta nesses dois modos. É possível fazer bons trabalhos de campo por meio de entrevistas e trabalhos de campo ruins em um ambiente de imersão na língua estudada. E vice-versa. Além disso, os autores chamam a atenção para o fato de que o trabalho de campo em linguística em ambientes não exóticos é muito mais comum do que se parece quando se lê os textos sobre o assunto. Nesse sentido, o linguista de campo não é mais necessariamente aquela figura caricata do pesquisador que desbrava a selva em busca de dados linguísticos.

Segundo Crowley (2007), por muito tempo os pesquisadores em linguística foram divididos em dois tipos: os linguistas pés-sujos, que fazem os trabalhos de campo, e não possuem nenhuma sofisticação teórica e os linguistas de poltrona que fazem suas teorias baseadas em dados introspectivos e totalmente fora da realidade (termos do autor). Crowley (2007) afirma que essa dicotomia caricata não existe.

É possível que o trabalho de campo se realize na comunidade dos falantes ou, ainda, 
em um outro ambiente, em que os linguistas possam se encontrar com os falantes. Muitas vezes, por questões técnicas, a segunda possibilidade é a preferida. É o caso de trabalhos de campo comuns em fonética e fonologia que envolvem gravações com o uso de equipamentos específicos que ficam na universidade. Embora esse não seja o nosso caso, os trabalhos de campo com os falantes de Karitiana têm sido feitos tanto na Universidade de São Paulo, em São Paulo, como em Porto Velho, em Rondônia.

Logo, o termo trabalho de campo tem, atualmente, um sentido muito mais amplo. Segundo Chelliah e de Reuse (2011), o trabalho de campo em linguística descritiva compreende tanto a coleta de dados por meio de interação com os falantes em situações de uso natural da língua quanto por meio de entrevistas. Nesse sentido, o trabalho teórico e o prático não são mais excludentes. A próxima seção discutirá de que forma a teoria e a prática estão presentes na coleta de dados em pesquisa linguística.

\subsection{Teoria e Prática no Trabalho de Campo}

Há muitas formas de se coletar dados linguísticos, cada uma delas mais ou menos apropriada para um determinado tipo de investigação. Uma pesquisa que tenha como objetivo a investigação da fonologia de uma língua utiliza instrumentos e métodos que não são necessariamente adequados para uma investigação da sintaxe dessa língua. Um trabalho que pretenda investigar, por exemplo, os processos fonológicos do português brasileiro não pode reunir dados baseados apenas em textos escritos. Já um trabalho em sintaxe, que depende de evidências negativas (os dados agramaticais) não pode ser feito baseado apenas em dados espontâneos. Nesse sentido, no trabalho de campo, a teoria e a prática não podem ser desvincilhadas.

Essa não é, no entanto, uma postura compartilhada por todos os pesquisadores sobre o assunto. Abbi (2001), por exemplo, afirma que a teoria amarra as mãos do linguista de campo (termo da autora) no sentido de que ela limita a autonomia do pesquisador direcionando-o a uma coleta de dados limitada. Bowern (2008), por outro lado, assume que teoria e descrição não são mutuamente excludentes, e que a teoria é inerente à pesquisa. Não é possível fazer a descrição de uma língua sem a formulação de hipóteses sobre como ela funciona. Quando o pesquisador faz a descrição de uma língua, as escolhas dos dados e a metalinguagem das categorias revelam o emprego de uma teoria. 
A autora faz uma comparação interessante entre o trabalho do linguista de campo e o de Sherlock Holmes. O investigador muitas vezes pedia a seu companheiro Watson para fazer a descrição do cenário de um crime. Watson, inexperiente, tem problemas na tarefa porque não sabe exatamente o que o seu professor está pedindo. Holmes, nesse momento, intervém e chama a atenção de que só é possível fazê-lo se você possui uma hipótese, algo para procurar. Segundo Bowern (2008), o trabalho de campo envolve descobertas por meio de perguntas, mas o pesquisador precisa saber quais perguntas fazer. A experiência do linguista e o quadro teórico com a qual trabalha, seja ele de que tipo for, o ajudam a determinar quais perguntas elaborar e a fazer predições passíveis de serem testadas. Por exemplo, se um linguista está investigando uma língua e se depara com dados que parecem indicar que ela não possui quantificadores nominais (como é o caso do Karitiana, descrito no capítulo anterior), mas não está familiarizado com a literatura que apresenta a possibilidade de existência de línguas que possuem apenas quantificadores adverbiais (Partee et el. 1987 e Bach et al. 1995); sua descrição se tornaria muito mais árdua. O pesquisador pode ser forçado, por exemplo, a analisar os quantificadores adverbiais como nominais.

Nesse sentido, defendemos que não há diferença qualitativa entre descrever e analisar. A pesquisa que é chamada de descritiva não é possível de ser executada sem uma teoria e a que é considerada teórica não pode se desenvolver sem o uso de um corpus. Logo, o procedimento de coleta de dados utilizado nesta pesquisa está fortemente ligado ao paradigma teórico da Semântica Formal.

Esta seção discutiu de modo genérico as características de trabalho de campo de linguística. Mais especificamente, defendeu-se que teoria e prática estão intimamente ligadas nesse processo. A próxima seção procura ilustrar essas afirmações com exemplos apresentados de forma detalhada de como é esse procedimento.

\subsection{Semântica Formal e Elicitação Controlada}

Esta seção apresenta a metodologia utilizada para coleta dos dados de uma língua para a sua investigação nos moldes da Semântica Formal. O objetivo é mostrar que a elicitação controlada é o modo mais adequado para a coleta de sentenças acompanhadas de suas respectivas condições de verdade, o objeto de estudo do paradigma utilizado. O protocolo seguido é basicamente o que se encontra descrito em Matthewson (2004) e vem 
sendo empregado amplamente nos trabalhos de campo para coleta de dados linguísticos nesse modelo teórico. Conforme discutido anteriormente, o objetivo da Semântica Formal é investigar qual a contribuição de cada expressão linguística para as condições de verdade associadas a uma sentença (que revelam, segundo a teoria, o seu significado). Logo, a coleta de dados para pesquisa nesse paradigma teórico deve conduzir a sentenças acompanhadas de suas respectivas condições de verdade.

Um possível primeiro passo de aproximação aos dados de uma língua desconhecida é por meio de consulta do acervo de seus dados espontâneos, escritos ou não. Entretanto, nem sempre a língua que se quer investigar possui um acervo desse tipo. Além disso, esse passo é apenas para uma aproximação aos dados da língua. É impossível coletar as informações necessárias para um trabalho que faz uma investigação sobre as condições de verdade das sentenças apenas por meio do discurso espontâneo ou de textos. Os dados que podem ser obtidos pelo uso exclusivo desse método quase sempre não são suficientes pois não fornecem informações mais sutis ou dependentes do contexto que são cruciais para o estudo do significado das sentenças. A sentença em (82), por exemplo, foi retirada de uma narrativa oral em Karitiana traduzida para o português (Malosso 2003).

(82) a. Mynda, y'ete'et, ipibmam 'in tyym ikytop, iohynam pitat yj'a tykiri taka'a okot bypiitap tairisooty. Ty'ooman 'ej se'yp am'a tykiri.

'Sem pressa, a gente faz a quantidade de chicha, porque quando a gente faz muita chicha, acontece morte próxima'

b. Taso tapyot iataki pitat tyym, isoko'im tyym, kyj, i'a tykit imbodn.

'Todos fazem o moquém, amarram sem arrebentar o cipó. Se arrebentar, não é bom'

Suponha que queremos usar essas sentenças para estudar o modificador de grau pita(t) na língua, traduzido usualmente como 'muito' ${ }^{23}$. Pode-se observar que é impossível retirar informações acerca de seus usos possíveis e quais as condições de verdade das sentenças em que esse advérbio aparece a partir de sentenças como essas. Quanto à distribuição, é impossível retirar de dados como em (82) uma generalização sobre as possibilidade de uso de pita(t). A tradução em (82) por não apresentar segmentação

23 Conforme será discutido nesta tese, pita(t) tem um uso como modificador de substantivos e adjetivos como pita e um uso como modificador adverbial de predicados verbais como pitat. 
morfológica nem tradução palavra a palavra não dá pistas nem do tipo de sintagma que é modificado por pita(t). Conforme será discutido em detalhes no capítulo 4, essa é uma informação crucial para a investigação do significado do modificador. Para a investigação do significado, a deficiência desse tipo de dado se torna ainda mais dramática. Podemos ver que o que temos em (82) é apenas uma tradução adequada para o contexto em que pita(t) ocorreu nessa narrativa. Em (82b) não há nem a palavra 'muito' na tradução. Não conseguimos obter informações de outras situações em que essas sentenças poderiam ser usadas, ou ainda saber em que contextos elas não poderiam ser utilizadas adequadamente. Esse tipo de informação é essencial e não aparece nos corpora de discurso espontâneo.

Nesse sentido, o trabalho de campo com elicitação controlada é de suma importância para uma pesquisa como essa ${ }^{24}$. A elicitação controlada é um método de coleta de dados baseado em entrevistas que possui, basicamente, duas etapas: traduções e julgamentos de contextos. Nas duas etapas, Matthewson (2004) defende o uso de uma metalíngua como instrumento, uma língua que não seja a língua que está sendo investigada e que seja conhecida pelo pesquisador e pelo consultor ${ }^{25}$. A metalíngua utilizada em nossas pesquisas com os Karitiana é o português brasileiro.

A primeira etapa da elicitação envolve a coleta de traduções, que representam ferramentas importantes para o trabalho de campo. No entanto, elas devem ser utilizadas como pistas e não como resultados, constituindo a menor parte do trabalho de campo. A autora defende que sempre sejam pedidas traduções de sentenças completas e nunca de palavras ou sintagmas menores que uma sentença. Isso porque não é fácil, para o consultor, isolar a sequência que é relevante em sua língua ou muitas vezes a tradução pode não ser possível porque uma sequência de três palavras em uma língua pode ter apenas uma em uma outra.

O exemplo (83a) ilustra esse fato. Em Karitiana, a palavra aniki pode ser traduzida para o português como 'estar com frio'. (83b) traz um exemplo em inglês em que ocorre o contrário: duas palavras give up traduzida para uma ('desistir'). Veja que alguém que tentasse traduzir give up para o português traduzindo palavra a palavra chegaria a algo sem sentido, como 'dar acima'.

24 No entanto, nem todos os linguistas aceitam que a elicitação controlada seja um bom método de coleta de dados. Entre os autores que rejeitam o tipo de elicitação utilizada nesta pesquisa e descrita nesta seção estão Schütze (1996), Dimmendaal (2001) e Mithun (2001) (apud Matthewson 2004).

25 Bowern (2008) prefere o termo consultor a informante, pois defende que consultor tem a conotação de um especialista que é consultado sobre um tópico específico. Estamos seguindo a sua sugestão no uso do termo. 
a. aniki

(Karitiana)

'estar com frio'

(Landin, D. 1984)

b. give up

'desistir'

Para se fazer as traduções de sentenças, segundo Matthewson (2004), deve-se partir de cenários descritos na metalíngua que descrevem o contexto no qual está inserida a sentença que se quer traduzir para depois o pesquisador falar a sentença. Se a sentença vier antes, ela pode induzir o consultor a contextos diferentes daquele que o pesquisador quer elicitar. A autora afirma que a influência da metalíngua sobre o consultor é desprezível, uma vez que ela exerce tanta influência quanto exerceria a língua objeto se o contexto fosse criado nessa língua. As sentenças de que se parte na metalíngua devem ser gramaticais e deve-se assumir que as sentenças obtidas na língua objeto são também gramaticais.

Por exemplo, para se coletar a tradução da sentença em Karitiana para 'O ovo caiu', deve-se proceder da seguinte forma:

(84) Tem um ninho em cima de uma árvore. Eu quero dizer que o ovo que estava no ninho caiu; como eu falo em Karitiana $O$ ovo caiu?

No entanto, nem todas as pistas semânticas de que se necessita aparecem diretamente nas traduções. Quando se pede, por exemplo, a tradução de uma sentença em português para o Karitiana, a sentença obtida pode não corresponder exatamente à sentença em português. Pode acontecer de a sentença em Karitiana poder ser usada em situações em que a sentença em português não é apropriada e vice-versa, ou seja, as sentenças podem não ter as mesmas condições de verdade. Por exemplo, ao se pedir em Karitiana uma tradução para $O$ Inácio esperou muito obteve-se a seguinte sentença:
Inácio Ø-naka-kydn-Ø kanda-t.
Inácio 3-DECL-esperar-NFUT muitas.vezes-ADV
'O Inácio esperou muito' 
Em Karitiana, essa sentença só pode ser usada para cenários em que o Inácio esperou muitas vezes. Ela não é apropriada para descrever um cenário em que o Inácio esperou uma vez por muito tempo. Já a sentença em português 'O Inácio esperou muito' é apropriada nesse contexto. Uma vez que a diferença entre iteração e duração é extremamente importante para o estudo dos advérbios de quantificação (Doetjes 2007), o método de tradução, apenas, não é suficiente.

Essa impossibilidade de tradução um para um é descrita em Quine (1960) como a indeterminação da tradução radical. A tradução completa de uma língua para outra é impossível uma vez que cada sistema linguístico tem sistemas de significados distintos. Esses sistemas não podem ser alcançados ostensivamente, ou seja, não é possível simplesmente apontar no mundo uma referência na tentativa de reproduzir o significado de uma expressão. Essa característica ficou conhecida na literatura como a inescrutabilidade da referência. $\mathrm{O}$ exemplo de Quine (1960) é a palavra gavagai. Se um linguista estudando um língua percebe que os falantes parecem utilizar a palavra gavagai nas mesmas situações em que utilizaríamos a palavra coelho em português, ele não pode assumir que gavagai e coelho se referem à mesma coisa. Em cada língua, as palavras podem agrupar conceitos distintos. No exemplo do Karitiana em (85), vimos que muito do português, embora possa ser traduzido em alguns casos por kanda(t) do Karitiana, esse não é sempre o caso. Muito pode ser usado para descrever um contexto em que houve um evento de espera muito longo, contexto em que kandat não é adequado.

Desse modo, a segunda etapa da elicitação controlada sugerida por Matthewson (2004) é crucial para os trabalhos que investigam as condições de verdade das sentenças. Trata-se da coleta de julgamentos do valor de verdade das sentenças em contextos particulares. O guia sugerido pela autora para a etapa dos julgamentos segue o modelo sugerido para as traduções: o pesquisador fala na metalíngua primeiramente o contexto no qual está inserida a sentença e, em seguida, pronuncia a sentença na língua objeto. O objetivo é checar a aceitabilidade da sentença no contexto sugerido.

$\mathrm{Na}$ etapa de elicitação de julgamentos, o uso do contexto é fundamental. Mais até do que para as traduções. É impossível questionar quais os valores de verdade de uma sentença sem apresentação de um contexto. A relação da sentença com o contexto pode ser de dois tipos: 
a. Se um falante aceita a sentença $\mathrm{S}$ em um contexto $\mathrm{C}, \mathrm{S}$ é verdadeira em $\mathrm{C}$.

b. Se uma sentença $\mathrm{S}$ é falsa em um contexto $\mathrm{C}$, o falante vai rejeitar $\mathrm{S}$ em $\mathrm{C}$.

Nota-se que, se o consultor rejeita uma sentença em um contexto, isso não é suficiente para se assumir que a sentença é falsa. Pode acontecer de a sentença ser verdadeira, mas ser infeliz nesse contexto. A infelicidade de uma sentença pode estar associada a falhas de pressuposição ou inverossimilhanças pragmáticas. Por exemplo, um fato que surgiu da coleta com o Karitiana ilustra um caso como esse. Na coleta de dados da língua, a primeira versão do dado (87) abaixo, com mulher e pegar água era com homens e não com mulheres. A sentença foi rejeitada sem nenhum motivo ligado a sua gramaticalidade ou a suas condições de verdade. O que ocorre nesse caso é uma impossibilidade ou improbabilidade pragmática: não são os homens que buscam água na aldeia Karitiana, mas as mulheres. Nesse caso, foi elaborada uma sentença infeliz.

jonso $\varnothing$-naka-ot- $\varnothing \quad$ ese.

mulher 3-DECL-pegar-NFUT água

'As mulheres pegaram água'

No caso específico do estudo dos modificadores de grau em Karitiana em dados como em (88), o procedimento para a coleta dos julgamentos de verdade dessa sentença está descrito em (89):

$$
\begin{aligned}
& \text { Taso i-pytim'adn-<a }>\text {-t pita- }{ }^{26} \text {. } \\
& \text { homem PART-trabalhar-ABS pita-ADV } \\
& \text { 'O homem trabalhou muito' }
\end{aligned}
$$

(89) a. Em um contexto em que o homem trabalhou muitas vezes esta semana ou este mês, eu posso usar a sentença Taso ipytim'adnat pitat?

b. $\quad$ Em um contexto em que o homem trabalhou uma vez por muito tempo esta semana, eu posso usar a sentença Taso ipytim'adnat pitat?

\footnotetext{
26 Optamos por não traduzir pita na glosa por se tratar de uma palavra que pode ter várias traduções em português. O significado de pita(t) será longamente debatido no capítulo 4.
} 
c. Em um contexto em que o homem trabalhou uma vez, mas bastante intensamente esta semana, eu posso usar a sentença Taso ipytim'adnat pitat?

Nota-se que os contextos usados na elicitação controlada desse dado foram criados levando-se em conta a teoria na qual ele está embasado. Para o estudo dos quantificadores adverbiais de grau é crucial a diferença entre iteratividade, duração e intensidade (Doetjes 2007) representados em (89a), (89b) e (89c) respectivamente ${ }^{27}$. Vemos aqui que o quadro teórico adotado é absolutamente indispensável na composição do corpus de uma pesquisa como esta. A teoria neste trabalho não só organiza e analisa os dados, mas determina o tipo de corpus a ser coletado, uma vez que ela é crucial para as escolhas dos dados e contextos elicitados.

Esta seção descreveu de que modo é feita a elicitação controlada, uma ferramenta metodológica indispensável para o trabalho de campo em Semântica Formal. A próxima seção trata de algumas questões éticas envolvidas nos trabalhos de campo em linguística.

\section{3 Ética}

Esta seção pretende discutir brevemente a questão ética dos trabalhos de campo em linguística. Uma vez que se trata de uma prática que envolve seres humanos, ela suscita uma discussão a respeito de problemas éticos envolvidos. A questão ética é uma questão muito complexa que envolve muitas esferas, inclusive filosóficas (Rice 2012). O que se pretende fazer nesta seção não é discutir aprofundadamente a questão, mas apenas descrever e justificar o modo pelo qual essas questões estão sendo encaradas em nossas pesquisas.

Segundo Bowern (2008), há dois tipos de questões éticas com as quais o linguista de campo tem que lidar. A primeira delas refere-se ao campo legal, associado ao comité de ética da Universidade em que se realiza a pesquisa ou a suas agências de fomento. O segundo tipo está associado ao comportamento ético do linguista em relação à comunidade da língua estudada.

Quanto à primeira esfera, a literatura sobre os trabalhos de campo em linguística falam muito pouco sobre a questão legal. Pouco se diz sobre como os órgãos gestores avaliam as pesquisas em linguística de campo. Nos Estados Unidos, a Linguistic Society of America

27 Essa questão será esclarecida ao longo da tese. 
possui uma Declaração de Ética (Ethics Statement) para conduzir trabalhos de campo com coleta de dados linguísticos. Essa declaração descreve a responsabilidade de um pesquisador que realiza trabalho de campo em linguística em cinco esferas.

A primeira esfera diz respeito à responsabilidade com os indivíduos que participam da pesquisa. O pesquisador precisa obter o consentimento dos indivíduos que serão consultados e os participantes devem ter o direito de controlar até que ponto a coleta pode ser gravada e quais dados pessoais podem ser divulgados. A participação deve ser totalmente voluntária, no sentido de não ser obrigatória, mas o pesquisador deve considerar uma compensação de algum tipo para o tempo e o esforço despendido pelo indivíduo consultado. A segunda esfera de responsabilidade envolve a participação da comunidade dos indivíduos consultados. O linguista deve levar em conta não apenas os indivíduos que fazem parte de sua pesquisa, mas também a sua comunidade, suas necessidades e expectativas em relação ao trabalho realizado. A terceira esfera de responsabilidade diz respeito à relação do pesquisador com seus alunos e colegas. A declaração afirma que a contribuição de alunos e colegas deve ser reconhecida e os conflitos de interesse devem ser evitados na prática do trabalho de campo. A declaração cita ainda a responsabilidade que o linguista deve ter com as agências de fomento que financiam os trabalhos de campo. O pesquisador deve ter um alto grau de profissionalismo e realizar um trabalho que possa ajudar pesquisadores que venham a trabalhar com aquela comunidade/língua no futuro. Por fim, a declaração cita a responsabilidade com a sociedade, lembrando das implicações políticas e sociais que podem resultar um trabalho de campo em linguística.

No Brasil, no entanto, não há nada semelhante a isso publicado pela Abralin (Associação Brasileira de Linguística). As agências de fomento brasileiras como o CNPq Conselho Nacional de Desenvolvimento Científico e Tecnológico - apresentam diretrizes baseadas na Comissão Nacional de Ética em Pesquisa (CONEP) criada pela Resolução do CNS 196/96, que está diretamente ligada ao Conselho Nacional de Saúde (CNS). Ou seja, essas diretrizes não estão adaptadas para avaliar projetos de pesquisa em linguística. Nas diretrizes do CNPq encontra-se ainda uma referência à FUNAI - Fundação Nacional do Índio - quanto à permanência e trânsito por áreas indígenas, ainda assim pouco específica para o caso de pesquisadores linguistas. Nas universidades, as pesquisas contam com avaliações de Comitês de Ética em Pesquisa em cada instituto. No entanto, é raro encontrar um Comitê de Ética em um instituto de Ciências Humanas. Eles estão, quase sempre associados aos 
institutos de Ciências Biológicas.

A segunda esfera ética, que diz respeito ao comportamento ético do linguista em relação à comunidade da língua estudada também é bastante complexa, pois o comportamento ético é dependente da cultura. O que é ético em uma cultura pode não ser em outra (Bowern 2008). Essa não é, no entanto, uma desculpa para se ignorar as questões éticas. É possível seguir uma pesquisa ética seguindo um princípio geral de maximizar os benefícios e minimizar os riscos para as partes envolvidas (pesquisadores e falantes) (Rice 2012 e Bowern 2008).

Rice (2012) sugere que o primeiro passo seja de negociação. É preciso primeiramente explicar aos consultores o trabalho que se pretende fazer, o que se pretende estudar. É preciso considerar que a comunidade espera algo do linguista e é essencial se discutir como o trabalho pode ser realizado para que todos se beneficiem com ele. Nesse ponto, é essencial conhecer o histórico dos trabalhos de linguistas na comunidade para compreender a percepção que os falantes têm do linguista de campo. Muitas vezes, o papel do linguista se confunde com o dos colonizadores, dos missionários ou dos agentes de saúde (Chelliah e de Reuse 2011). Além disso, Rice (2012) e Bowern (2008) chamam a atenção para o fato de que a negociação deve ser constante, pois é sempre possível haver mudanças nas decisões da comunidade, e elas devem ser sempre respeitadas.

Isso foi precisamente o que foi feito em abril de 2008 quando o grupo de pesquisa de língua Karitiana visitou a sua aldeia em Rondônia. Estavam presentes as professoas Luciana Storto e Ana Müller e três alunos de pós-graduação, Thiago Coutinho-Silva, Andrea Carvalho e eu. $\mathrm{Na}$ ocasião, conversamos com as lideranças e com membros da comunidade em uma assembleia que explicou o trabalho que vinha sendo feito, bem como apresentou uma pequena cartilha contendo textos escritos pelos pesquisadores do Karitiana sobre assuntos diversos da gramática da língua. Desde então, o trabalho conjunto vem se desenvolvendo com constantes renegociações. Em abril de 2013, uma outra reunião foi realizada na aldeia, a fim de discutir os resultados obtidos desde 2008 e de reafirmar a parceria entre pesquisadores e consultores falantes de Karitiana para o estudo da língua. Estiveram presentes as professoras Luciana Storto e Ana Müller e três alunos de pós-graduação, Ivan Rocha, Karin Vivanco e eu. Esse exemplo ilustra os processos de negociação e renegociação envolvidos em uma pesquisa como esta.

Uma parte importante das negociações diz respeito ao modo de pagamento aos 
consultores pelo tempo dispensado no trabalho. Todos os trabalhos consultados sobre o assunto foram unânimes em afirmar que é preciso que se estabeleça alguma compensação aos consultores, que não precisa ser necessariamente em dinheiro, mas pode ser com algum tipo de benefício que auxilie os participantes e a sua comunidade. Se em dinheiro, o modo de pagamento deve ser adequado à comunidade e deve ser baseado no valor que é geralmente aceitável naquela cultura (Rice 2012 e Bowern 2008).

Rice (2012) afirma que estabelecer um meio e um valor de pagamento é parte de demonstração de respeito à comunidade cuja língua é estudada. Bowern (2008) diz ainda que uma compensação apropriada pelo tempo dispensado formaliza a relação entre o pesquisador e o consultor e encoraja os envolvidos a trabalhar de modo sério, considerando a coleta de dados como um trabalho que precisa ser feito, e não um passatempo. Os trabalhos de campo que têm sido feitos com os Karitiana segue esse protocolo e o modo de pagamento faz parte da negociação entre linguistas e consultores. Esta seção mostrou brevemente algumas diretrizes para a condução ética do trabalho de campo em linguística.

\subsection{Considerações Finais}

Esta seção tratou de algumas questões envolvidas em trabalhos de campo para coleta de dados linguísticos. Primeiramente, o trabalho desmistificou a figura do linguista de campo mostrando que a coleta de dados linguísticos não está associada, necessariamente a ambientes exóticos. Além disso, a própria existência de um linguista que é apenas um linguista de campo foi questionada. Uma vez que se defendeu que não há uma diferença significativa entre descrever e analisar, não faz sentido separar os linguistas entre pesquisadores teóricos e práticos.

Por meio de exemplos retirados da pesquisa sobre quantificadores adverbiais em Karitiana, o capítulo mostrou que o paradigma teórico no qual se insere uma pesquisa linguística é o que determina o protocolo de formação do corpus. A Semântica Formal, por exemplo, como trabalha associando sentenças a suas condições de verdade, motiva uma coleta de dados que leve em conta traduções de sentenças e julgamentos dos seus valores de verdade.

Por fim, apresentou-se uma reflexão sobre algumas questões éticas que estão necessariamente envolvidas em um trabalho com seres humanos. Os tópicos foram 
brevemente discutidos com base na negociação e renegociação, tema recorrente em todos os trabalhos sobre o assunto consultados. A próxima seção discute algumas questões metodológicas que podem surgir de uma pesquisa que investiga o significado.

2

A Divisão de Trabalho entre a Semântica e a Pragmática: Consequências Metodológicas

O objetivo desta seção é discutir algumas questões pragmáticas envolvidas na análise semântica da quantificação adverbial em Karitiana. Mais especificamente, pretende-se mostrar como inferências pragmáticas podem ajudar a analisar apropriadamente o significado de advérbios como kanda(t) na língua em sentenças como em (90).
jonso Ø-naka-ot-
kanda-t
ese.
mulher 3-DECL-buscar-NFUT muitas.vezes-ADV água
'Mulher buscou água muitas vezes'

A seção está dividida da seguinte forma: a seção 2.1 apresenta uma breve descrição da Semântica e Pragmática enquanto teorias de análise do significado. Em seguida, a seção 2.2 apresenta o fenômeno da quantificação adverbial com o advérbio kanda(t) em Karitiana. A seção 2.3 mostra qual a proposta que defendemos para a análise semântica de kanda(t) e a compara com outras propostas semelhantes no que tange à divisão de trabalhado entre a Semântica e a Pragmática em análises do significado. A seção 2.4 explora quais a consequências desse tipo de divisão de trabalho para a metodologia de coleta de dados de uma língua para uma pesquisa como esta. A seção 2.5 apresenta, por fim, as considerações finais.

\subsection{Semântica e Pragmática}

O objetivo desta seção é apresentar, brevemente, duas áreas envolvidas em aspectos do significado das línguas naturais: a Semântica e a Pragmática. Ambas as áreas são essenciais para o entendimento de como o significado é construído. Nos últimos 40 anos observa-se um esforço dos pesquisadores para uma integração das teorias sobre essas áreas (McNally, no prelo). 
Todo trabalho que trata da complexidade do significado é, de uma forma ou de outra, um trabalho de divisão entre significado convencional e significado contextual (McNally, no prelo). A Semântica é uma teoria voltada para o estudo do significado convencional, do conjunto de códigos sistematizados na gramática de uma língua. A Pragmática, por sua vez, interessa-se pelo significado dependente do contexto de uso; pelo conjunto de inferências não lógicas derivadas com base nesses códigos (Ariel 2008). Nesse sentido, investigar quais aspectos do significado são parte do código e quais são derivados via inferência é avaliar a divisão de trabalho entre Semântica e Pragmática na constituição do significado.

Nesta tese, assumimos uma teoria pragmática neogriceana (Grice 1989). Segundo essa proposta, sempre que possível, deve-se atribuir um significado único para uma forma linguística e derivar as outras interpretações por meio da Pragmática. Um exemplo clássico desta proposta é a análise dos numerais. Segundo uma proposta neogriceana, um numeral como três significa pelo menos três e sua interpretações de exatamente três é derivada via pragmática.

Essa proposta explica porque, em certos casos, um numeral deve ser interpretado como pelo menos $n$, como (91a); e, em outros, como exatamente $n$ (exemplo 91b).

(91) a. Estou precisando de dinheiro emprestado. Você tem vinte reais?

b. O João tem três filhos.

Em (91a), não é reevante saber quando dinheiro a pessoa tem, mas apenas se ela tem pelo menos vinte reais. Em (91b), por outro lado, o falante quer informar que o João tem exatamente três filhos. O Princípio de Cooperação de Grice (1975) explica como a interpretação exatamente $n$ é derivada da denotação pelo menos $n$. Segundo esse princípio, a comunicação se baseia em máximas conversacionais. Uma dessas máximas é a máxima da quantidade, segundo a qual a contribuição do falante deve ter a quantidade exata de informação necessária para os propósitos da conversa ${ }^{28}$.

28 Para uma discussão das outras máximas, ver Grice (1975). 
Máxima da Quantidade:

a. $\quad$ Faça da sua contribução tão informativa quanto necessária para os propósitos da comunicação;

b. Não faça sua contribuição mais informativa do que necessária.

Se, em (91b), o falante soubesse que o João tem mais de três filhos, ele falaria, porque a situação seria mais informativa e a informação é relevante para o contexto. Se o falante não disse que o João tem mais de três filhos, é porque o falante acredita que o João possui exatamente três filhos. A próxima subseção mostra um exemplo de análise neogriceana do significado, em que um significado único é atribuído a um item lexical em Karitiana, enquanto que certas leituras das sentenças em que ocorre são derivados via inferência.

\subsection{A Quantificação Adverbial com $k a n d a(t)$}

O objetivo desta seção é apresentar alguns aspectos da língua Karitiana que estão envolvidos na quantificação adverbial com o advérvio kanda(t). Esse assunto foi escolhido porque ilustra perfeitamente as questões semânticas e pragmáticas que podem surgir da análise de um quantificador em uma língua sem quantificadores de determinante, como o Karitiana. Em Karitiana, todos os sintagmas em posição de argumentos aparecem nus, sem determinantes, quantificadores ou classificadores. Eles não possuem morfemas de singular e plural e têm sempre denotação neutra para número. A sentença em (93) ilustra essa propriedade. Tanto um substantivo usualmente contável, como jonso 'mulher', quanto um tradicionalmente massivo como ese 'água' aparecem nus e denotam uma quantidade indeterminada.

Jonso Ø-naka-ot-Ø

ese.

mulher 3-DECL-buscar-NFUT água

'Mulher buscou água'

A sentença acima, quando utilizada com o advérbio kanda(t), exibe uma variabilidade de traduções para o português (possibilidades descritas em (95)). 
Jonso Ø-naka-ot- $\varnothing \quad$ kanda-t $\quad$ ese. $=(90)$

mulher 3-DECL-buscar-NFUT muitas.vezes-ADV água

'Mulher buscou água muitas vezes'

(95) Contextos apropriados para a sentença (94):
a. 'Muitas mulheres buscaram água'
b. 'Mulher buscou muita água'
c. 'Mulher buscou água muitas vezes'
d. 'Muitas mulheres buscaram muita água muitas vezes'

O que se observa nas situações descritas em (93) é que os nomes comuns em sentenças quantificadas com kanda(t) têm duas interpretações possíveis: (i) uma leitura mais fraca de um ou mais, uma quantidade indefinida, como em (93c); ou (ii) uma leitura mais forte de muitas mulheres, muita água, como em (93a), (93b) e (93d). A próxima subseção apresenta uma proposta de análise que defende o significado descrito em (i) para os nomes comuns em sentenças com kanda(t). A leitura (ii) será derivada via inferência pragmática.

\subsection{Proposta}

Nesta subseção, vamos ilustrar porque devemos adotar a proposta griceana de que sintagmas nominais em sentenças com kanda(t) tem a denotação mais fraca (a denotação neutra para número). Os exemplos em (92) e (94) mostraram que kanda(t) parece ter um comportamento ambíguo entre um quantificador nominal e um quantificador adverbial. Defendemos que kanda(t) é um quantificador adverbial exclusivo de eventos que sempre significa 'muitas vezes'. Na nossa proposta, as leituras de 'muitas mulheres', 'muita água' verificadas acima serão derivadas via inferência pragmática.

O principal argumento a favor dessa proposta é baseado nos contextos inapropriados para a sentença (94), descritos em (96): 

a. 'Muitas mulheres buscaram água uma vez'
b. 'Mulher buscou muita água uma vez'
c. 'Muitas mulheres buscaram muita água uma vez'

Além disso, a sentença (94) pode ser usada em contextos em que apenas uma mulher participou do evento e houve uma pequena quantidade de água, como mostram os contextos descritos em (97):

$$
\text { Contextos apropriados para a sentença (94): }
$$
a. 'Uma mulher buscou água muitas vezes'
b. 'Mulher buscou pouca água muitas vezes'

O contraste entre os contextos descritos em (95), (96) e (97) demonstra que a referência a muitas entidades é uma possibilidade em sentenças com kanda(t) (contextos de 95a a 95d), mas não uma necessidade (cntextos 97a e 97b); enquanto que a leitura de muitos eventos é uma obrigatoriedade (contextos de 96a a 96c). Isso indica que kanda(t) quantifica sempre sobre os eventos e as leituras possíveis de 'muitas mulheres' e 'muita água' são possíveis porque os sintagmas nominais na língua são neutros para número. Essas leituras são fornecidas por meio de inferências pragmáticas, isto é, elas podem ser inferidas em certos contextos, mas não fazem parte das condições de verdade da sentença. A denotação da sentença (94) está descrita em (98):

$$
\llbracket \text { jonso nakaot kandat ese } \rrbracket=1 \text { sse } \exists \mathrm{E} . \forall \mathrm{e} \in \mathrm{E}[\operatorname{buscar}(\mathrm{y})(\mathrm{e}) \& \text { água (y) \& }
$$

$$
\text { Agente }(\mathrm{x})(\mathrm{e}) \& \text { mulher }(\mathrm{x}) \&|\mathrm{E}|>\mathrm{N}]^{29}
$$

Em palavras, o que está descrito em (98) é que a denotação da sentença jonso nakaot kandat ese 'Mulher buscou água muitas vezes' é o valor de verdade 1, ou seja, o verdadeiro, se e somente se, existe um evento, este é um evento de pegar água, o agente deste evento é mulher e a cardinalidade do evento é maior que um normal $(\mathbf{N})$ determinado contextualmente.

\footnotetext{
29 Na denotação verbal estamos seguindo Kratzer (1986) que considera que apenas o argumento tema é um verdadeiro argumento do verbo. $\mathrm{O}$ argumento externo é inserido via predicação secundária pelo predicado Agente. Ver descrição no capítulo 1 para mais detalhes.
} 
A restrição de cardinalidade representa a contribuição semântica de que muitos eventos ocorreram. A próxima subseção compara a proposta apresentada acima com propostas semelhantes encontradas na literatura acerca da divisão de trabalho entre a Semântica e a Pragmática nos estudos do significado.

\subsubsection{Comparação com Propostas Semelhantes}

O objetivo desta subseção é apresentar uma comparação com outros casos de divisão de trabalho entre Semântica e Pragmática (neogriceana) encontrados na literatura. Em primeiro lugar, há semelhanças entre a proposta defendida nesta seção e a proposta apresentada por neogriceanos para a conjunção disjuntiva ou. Essa conjunção possui dois usos, um associado a uma leitura mais fraca (leitura e/ou), ilustrada em (99a); e outro representado por uma leitura mais forte, a leitura exclusiva, ilustrado em (99b).

$$
\begin{aligned}
& \text { a. Para se aposentar, é preciso ter } 65 \text { anos ou } 35 \text { anos de contribuição. } \\
& \text { b. } \quad \text { Você tem direito a uma sobremesa, escolha gelatina ou fruta. }
\end{aligned}
$$

A interpretação mais saliente da sentença (99a) é de que para se aposentar é preciso ter 65 anos de idade ou 35 anos de contribuição, mas não há problemas se a pessoa possuir as duas propriedades. Já a sentença (99b) tem como leitura mais saliente que cada cliente deve consumir gelatina ou fruta, exclusivamente; não podendo optar pelas duas sobremesas.

A análise de Horn (1972) para a conjunção ou é colocar na Semântica a denotação mais ampla (inclusiva) e derivar via inferência a noção exclusiva (mais restrita). Uma proposta semelhante é oferecida para o most 'a maioria' do inglês. Most possui uma leitura mais fraca que significa algo como 'mais da metade' (sentença em (100a)) e uma leitura mais forte de 'mais da metade, mas não todos' (sentença (100b)). 
a. Most students like Semantics ${ }^{30}$.

'A maioria dos alunos gosta de Semântica'

b. Most students were approved.

'A maioria dos alunos foi aprovada'

No caso de most, a proposta é semelhante à apresentada acima para a conjunção disjuntiva. Horn (1972) defende que se coloque na Semântica a denotação mais ampla (leitura mais fraca) e a leitura mais forte deve ser derivada via implicatura conversacional em alguns contextos via máxima de quantidade de Grice (1975). Ou seja, o falante não deve falar mais nem menos do que o necessário. Por exemplo, em (100b) acima, pode-se inferir que a maioria dos alunos, mas não todos, foi aprovada; porque se fosse esse o caso, o falante proferiria Todos os alunos foram aprovados. Uma vez que ele não disse uma sentença que seria mais informativa no contexto, essa sentença é falsa ou desconhecida do falante.

Vale notar que, embora haja uma semelhança com os casos de quantificação adverbial com kanda(t), em sentenças com most, há uma derivação via competição com um quantificador mais forte: todo. Em Karitiana, no entanto, não há uma estratégia linguística no domínio nominal para se expressar 'pouco'. Logo, as leituras representadas por 'muitas mulheres' e 'muita água' no domínio nominal em sentenças com kanda(t) não devem ser derivadas via competição, como no caso de most. A semelhança entre os dois casos está na proposta geral de se considerar como parte da Semântica o significado mais abrangente, e derivar o significado mais restrito via inferências pragmáticas. Por conta disso, estamos utilizando o termo mais geral inferência pragmática em vez de implicatura conversacional. As implicaturas conversacionais foram investigadas por Grice (1975) e estão diretamente ligadas a intenções do falante. No caso de kanda(t) que está sendo analisado nesta seção, o significado derivado não está necessariamente associado a intenções do falante, mas a possibilidades disponíveis pela forma lógica da sentença.

\footnotetext{
30 Os exemplos foram mantidos em inglês, pois a proposta foi feita com base nessa língua. A diferença de leitura não é totalmente clara em português. (99a) também parece ter a leitura forte em português. No caso da conjunção disjuntiva, ela parece ser geral para todas as línguas, uma vez que se trata de um operador lógico.
} 


\subsection{Caminhos da Proposta}

Diante da discussão apresentada, os desdobramentos da análise podem seguir diferentes caminhos. O primeiro deles é buscar investigar em Karitiana qual a leitura mais relevante, mais saliente ou preferencial de sentenças com kanda(t). Trabalhos que lançam mão da noção de inferência pragmática usualmente discutem quais as leituras preferenciais e que contextos contribuem para as inferências. Esse tipo de investigação com as sentenças contendo kanda(t) é um trabalho para pesquisa futura dada a dificuldade de se fazer uma pesquisa como essa em uma língua parcialmente descrita da qual não somos falantes nativos.

Um segundo desdobramento que pode ser explorado é como a diferença entre leituras disponíveis e canceláveis interferem na metodologia dos trabalhos de campo para a coleta de dados da língua. A próxima subseção apresenta algumas reflexões sobre a metodologia de coletas de dados discutida na seção 1 deste capítulo e sobre como a divisão de trabalho entre Semântica e Pragmática interfere no procedimento.

\subsubsection{Metodologia}

O objetivo desta seção é retomar e discutir as características do protocolo de elicitação controlada defendido em Matthewson (2004) para coletar dados linguísticos para uma pesquisa em Semântica Formal. A seção 1 deste capítulo mostrou que assumimos, juntamente com a autora, que é impossível coletar as informações necessárias para um trabalho que faz uma investigação sobre o significado das sentenças apenas por meio do discurso espontâneo ou de textos. Os dados que podem ser obtidos pelo uso exclusivo desses métodos muitas vezes não são suficientes, no sentido de que não possuem as informações necessárias para uma análise explícita do significado das sentenças. O pesquisador necessita de evidências negativas bem como de detalhamentos contextuais que são cruciais para esse tipo de investigação, e não são possíveis de serem obtidos por meio de textos ou transcrições de fala espontânea. Assim, a elicitação controlada é uma ferramenta metodológica indispensável para o trabalho de campo em Semântica Formal, que precisa ter acesso ao significado das sentenças.

Matthewson (2004) descreve dois tipos de elicitação: pedindo traduções e pedindo julgamentos. O guia sugerido pela autora para os julgamentos é o seguinte: o pesquisador fala 
na metalíngua primeiramente o contexto no qual está inserida a sentença e, em seguida, a sentença na língua objeto. Para a elicitação de julgamentos, o uso do contexto é fundamental. Mais até do que para as traduções. É impossível questionar sobre os valores de verdade e de felicidade de uma sentença sem apresentação de um contexto. A relação da sentença com o contexto pode ser de dois tipos:

(101) a. Se um falante aceita a sentença $S$ em um contexto $C, S$ é verdadeira em $C$.
b. Se uma sentença $S$ é falsa em um contexto $C$, o falante vai rejeitar $S$ em $C$.

A relação entre sentença e contexto descrita em (101a) afirma que se um falante aceita uma sentença em determinado contexto é porque a sentença é verdadeira naquele contexto. No entanto, isso não garante que o contexto sugerido e aceito seja o único contexto que representa as condições de verdade da sentença, ou seja, o seu significado (para o paradigma teórico adotado nesta tese). Pode-se ter elaborado um contexto mais amplo que inclua mais do que as condições de verdade da sentença. A sentença abaixo do português ilustra um caso em que não há correspondência total entre o contexto e as condições de verdade da sentença.

(102) Tem crianças na piscina do prédio.

Se a sentença em (102) acima fosse elicitada no seguinte contexto:

(103) Neste momento, há muitas crianças na piscina do prédio, eu posso proferir a sentença Tem crianças na piscina do prédio?

A sentença é verdadeira nesse contexto, mas ela descreve um cenário menor do que o descrito no contexto. A sentença também seria verdadeira em um contexto com poucas crianças. Em um outro contexto, forçando um pouco mais o argumento, em que tem crianças na piscina do prédio e o céu é azul, a sentença (102) também poderia ser utilizada; embora mais uma vez o contexto possua mais informações do que se veicula no significado da sentença. Responder a essas questões parece simples em exemplos como o de (102), mas elas não são nada triviais quando se trata de uma outra língua ou ainda de expressões mais 
complexas mesmo em português.

Por conta disso, propomos um passo a mais nas fases descritas no protocolo de Matthewson (2004). É preciso elicitar os contextos limítrofes para que se chegue ao contexto mínimo que representa as condições de verdade da sentença. Os contextos limítrofes são aqueles em que são apresentadas situações que ajudem a delimitar as informações contidas no significado da sentença. Ou seja, são aqueles que procuram explicitar o que realmente faz parte das condições da verdade de uma sentença (sua Semântica) e o que é significado derivado de seu uso (Pragmática). Os contextos limítrofes para o exemplo com advérbio kanda(t) de mulher buscando água seriam algo do tipo:

(104) a. O contexto é o seguinte: Tinha uma mulher só, e ela foi no rio pegar água sozinha, eu posso usar a sentença jonso nakaot kandat ese?

b. $\quad$ O contexto é o seguinte: Tinha uma mulher só, e ela foi no rio pegar água sozinha, ela só tinha um copinho, não tinha um balde, ela foi várias vezes, mas ainda assim pegou pouca água no total, eu posso usar a sentença jonso nakaot kandat ese?

O contexto (104a) tem como objetivo testar se a leitura de muitas mulheres em sentenças com kanda(t) é uma obrigatoriedade, ou seja, se ela faz parte do significado da sentença, ou se é derivada pragmaticamente em alguns contextos. A resposta obtida para a pergunta (104a) foi sim; o que indica que o sintagma jonso na sentença jonso nakaot kandat ese tem denotação neutra para número, podendo indicar, uma, algumas ou mesmo muitas mulheres. O contexto (104b) tem o mesmo objetivo, mas com relação ao tema ese 'água'. A resposta obtida também foi sim; confirmando a hipótese de que o advérbio kanda(t) quantifica apenas sobre os eventos da sentença, não tendo efeitos semânticos sobre os sintagmas nominais das sentenças. 


\subsection{Considerações Finais}

Esta seção mostrou que a divisão de trabalho entre a Semântica e a Pragmática é importante em um trabalho que pretende analisar o significado linguístico. No caso específico deste capítulo, procurou-se mostrar que kanda(t) deve ser analisado como um quantificador exclusivo de eventos. As leituras das sentenças com multiplicação das entidades nominais devem ser derivadas via inferências pragmáticas.

Discutimos a relação de uma proposta como essa com a metodologia de elicitação controlada para a coleta de dados para a pesquisa. Defendeu-se que o protocolo utilizado para coleta deve ter um passo a mais de delimitação do contexto mínimo que representa as condições de verdade da sentença. Para tal, deve-se testar o que estamos chamando de contextos limítrofes. Trata-se de contextos que ajudam a delimitar o significado convencional das sentenças, explicitando aspectos que podem ser explicados via inferências pragmáticas. 


\section{Capítulo 3}

\section{Sintagmas Verbais}

\section{Introdução}

O objetivo deste capítulo é discutir algumas propriedades dos sintagmas verbais em Karitiana. O capítulo está dividido em duas grandes seções. A primeira seção trata da distinção télico e atélico e das classes acionais em Karitiana. Seu objetivo principal é estabelecer a pertinência de se classificar os predicados verbais da língua segundo a telicidade e acionalidade. As teorias propostas para a acionalidade são apresentadas e algumas caracteríticas da língua são debatidas. Os testes tradicionalmente utilizados na literatura sobre o tema são aplicados em Karitiana em uma lista de verbos. A conclusão da seção é de que a classificação dos predicados segundo sua acionalidade é pertinente, desde que resguardadas as diferenças entre as línguas para as quais foi proposta e o Karitiana.

A segunda seção do capítulo discute duas propriedades semânticas dos predicados verbais em Karitiana, a cumulatividade e a contabilidade. Sua tese central é a de que o domínio verbal da língua é ao mesmo tempo cumulativo e contável, embora as duas noções pareçam teoricamente excludentes. Os dados da língua com pluracionalidade verbal e advérbios de frequência e de grau apoiam a proposta. A seção está dividida em duas partes. $\mathrm{Na}$ primeira subseção, são apresentados os dados da língua Karitiana que sugerem uma análise que leve em conta tanto a cumulatividade quanto a contabilidade. Mais especificamente, são discutidas a operação da pluracionalidade e a distinção entre advérbios de frequência e de grau na língua. A segunda parte apresenta uma discussão teórica com o objetivo de mostrar que essas duas propriedades são compatíveis, a despeito da sua aparente contradição.

O capítulo é relevante para esta tese porque esclarece a natureza semântica dos predicados verbais em Karitiana. A divisão dos predicados verbais segundo a sua acionalidade, mais especificamente a distinção télico-atpelico, será retomada no capítulo sobre a modificação de grau no domínio verbal, bem como a sua realação com a atomicidade e a contabilidade. 


\section{$1 \quad$ A Distinção Télico-Atélico e as Classes Acionais em Karitiana}

\subsection{Introdução}

O objetivo desta seção é discutir os sintagmas verbais em Karitiana segundo o que ficou conhecido na literatura como acionalidade ou aspecto lexical (o termo normalmente utilizado é a palavra em alemão aktionsart 'de ação'). A acionalidade diz respeito à natureza do evento codificada como uma propriedade inerente dos verbos. Ela não se confunde (ou não deve ser confundida) com as noções de tempo e de aspecto gramatical.

O tempo é uma marca que localiza o evento em uma linha temporal (Reinchenbach 1947). Reinchenbach (1947) formalizou a semântica temporal por meio de três momentos associados ao evento descrito e que devem ser localizados em uma linha temporal: o momento da fala (MF), o momento do evento (ME) e o momento da referência (MR). Na sentença (105a), por exemplo, o evento de abrir a porta marca o momento de referência e o evento de alguém pular a janela marca o o momento do evento (ambos no passado em relação o momento da fala). (105b) representa a relação entre os três momentos. O tempo enquanto marca linguística é caracterizado pela relação entre o momento da referência e o momento da fala. Na sentença abaixo, o tempo é passado porque o momento da referência é anterior ao momento da fala.

(105) a. Quando eu abri a porta, alguém pulou a janela.

b. $\quad \mathrm{MR}=\mathrm{ME}<\mathrm{MF}$

O momento da referência (MR) é simultâneo ao momento do evento (ME), e ambos são anteriores ao momento da fala (MF).

$\mathrm{O}$ aspecto, por sua vez, diz mais respeito às relacões temporais internas ao evento (Comrie 1985). Sua caracterízação mais comum refere-se à (in)completude do evento e ela pode ser descrita como a relação entre o momento do evento e o momento da referência. $\mathrm{O}$ aspecto é dito perfectivo quando o momento do evento está incluído no momento de referência; e o aspecto é imperfectivo quando o momento de referência está incluído no momento do evento ${ }^{31}$. As duas sentenças abaixo ilustram a diferença. Em ambas, o momento

\footnotetext{
31 As definiçõs foram bastante simplificadas. Para um estudo mais refinado do aspecto, ver Klein, W. (1994).
} 
de referência é descrito pela oração subordinada quando eu tinha 18 anos. Em (106a), com o verbo da sentença principal no perfectivo, o evento de morar no Rio de Janeiro (ME) está incluído no período de ter 18 anos. Em (106b) com o verbo da sentença principal no imperfectivo, o evento de morar no Rio de Janeiro estrapola o período de ter 18 anos, ou seja, inclui o momento de referência.

(106) a. Quando eu tinha 18 anos, eu morei no Rio de Janeiro.

a'. $\quad \mathrm{ME} \subseteq \mathrm{MR}$

O momento do evento (ME) está incluído no momento da referência (MR).

b. Quando eu tinha 18 anos, eu morava no Rio de Janeiro.

b'. $\quad \mathrm{MR} \subseteq \mathrm{ME}$

O momento da referência (MR) está incluído no momento do evento (ME).

A acionalidade, em contrapartida, está associada a propriedades internas do predicado e sua relação com o tempo. Do ponto de vista filosófico-ontológico, o fenômeno da acionadade é interessante porque explicita certas características da natureza do evento. A distinção das classes acionais é também importante do ponto de vista linguístico, como será explicitado mais abaixo, sobretudo porque explica a distribuição de certos adjuntos dos verbos e por que certas inferências só são válidas para sentenças que descrevem eventos de um certo tipo e não de outro.

O objetivo desta seção é mostrar, primeiramente, o percurso da teoria na definição dessas classes e de que modo elas são utilizadas em investigações semânticas do predicado verbal. A seção 1.2.1 apresenta a proposta clássica de Vendler (1957) de divisão dos verbos em accomplishments, achievements, atividades e estados. A seção 1.2.2 mostra o avanço da literatura em considerar a acionalidade como uma característica dos sintagmas verbais como um todo e não de verbos apenas. Na seção 1.2.3, a proposta de Rothstein (2004) que associa a telicidade à atomicidade é discutida. A segunda parte desta seção (subseção 1.3) discute as classes acionais em Karitiana. Os principais testes propostos na literatura são apresentados e debatidos. Seu objetivo é discutir se é possível averiguar se em Karitiana a divisão dos predicados verbais em accomplishments, achievements, atividades e estados é relevante, pois essa divisão será utilizada na análise da modificação de grau apresentada no próximo capítulo. 


\subsection{Teorias sobre Acionalidade}

\subsubsection{Vendler (1957)}

O objetivo desta subseção é apresentar a divisão dos predicados verbais em classes acionais proposta em Vendler (1957) ${ }^{32}$. Vendler (1957) foi o primeiro a sistematizar os verbos em diferentes classes de acordo com sua interação com o tempo. Sua divisão dos predicados verbais em accomplishments, achievements, atividades e estados, embora não seja incontroversa, é a base para as discussões posteriores acerca da acionalidade.

A primeira distinção importante feita pelo autor diz respeito à telicidade. $\mathrm{O}$ termo telicidade ficou consagrado na literatura sobre acionalidade, mas o artigo de Vendler de (1957) não chega a mencioná-lo. O autor aborda a noção em termos de clímax ou término lógico. Verbos télicos são aqueles que possuem um fim determinado, previsto (do grego telos, 'fim'). Assim, o sintagma desenhar um círculo tem um ponto final natural descrito pelo predicado. Correr, por outro lado, é um predicado que não tem um ponto final determinado lexicalmente. Por conta disso, faz sentido dizer terminar de desenhar um círculo, enquando não é coerernte dizer terminar de correr $^{33}$. Com esses exemplos, Vendler (1957) apresenta uma primeira distinção: atividades versus accomplishments. Verbos como correr são atividades porque representam um processo homogêneo que tem a mesma natureza como um todo ${ }^{34}$. Predicados como desenhar um círuculo perduram no tempo, do mesmo modo que verbos de atividade como correr, mas progridem em direção a um término determinado, seu telos. Esses verbos com duração e telos são os verbos de accomplishment.

A evidência oferecida pelo autor de que atividades e accomplishments são verbos de duração é que eles podem ser usados em tempos contínuos do inglês, como mostram os exemplos abaixo com o presente contínuo ${ }^{35}$ :

32 Como ficará claro na próxima seção, a distinção de Vendler (1957) é para verbos, sem considerar seus argumentos.

33 A não ser que interpretemos correr com um fim determinado, como em um contexto em que é frequente o João correr 10 quilômetros por dia, em que é possível dizer $O$ João terminou de correr querendo dizer que ele terminou de correr os 10 quilômetros.

34 Verbos de atividades também são chamados na literatura de processos (por exemplo em Bach 1986, Parsons 1990 e Verkuyl 1996).

35 Tempos contínuos em inglês são tempos formados pelo verbo auxiliar be 'ser' mais um verbo principal no progressivo (com -ing). 
$(107)$

a. I'm running.

ATIVIDADE

'Eu estou correndo'

b. I'm drawing a circle.

ACCOMPLISHMENT

'Eu estou desenhando um círculo'

(exemplos de Vendler 1957, p. 144)

A segunda distinção apresentada em Vendler (1957) é a distinção entre verbos de achievement como reconhecer e estados como amar. Diferentemente dos verbos de atividade e de accomplishment, verbos como reconhecer e amar não indicam eventos que se desnvolvem no tempo, mas verbos que podem ser verdadeiros a respeito de um sujeito em momentos de tempo determinados. A evidência oferecida pelo autor de que verbos de achievement como reconhecer e estativos como amar não são verbos de progressão temporal é sua incompatibilidade com o presente contínuo do inglês:

a. $\quad *$ I'm recognizing $i t^{36}$.

ACHIEVEMENT

'Eu estou reconhecendo'

b. * I'm loving it.

ESTADO

'Eu estou amando ${ }^{37 !}$

(exemplos de Vendler 1957, p. 144)

Verbos de achievement como reconhecer são predicados de momentos instantâneos, enquanto que verbos estativos como amar são predicados de momentos de tempo mais longos $^{38}$. Uma vez que são pontuais, os verbos de achievement são télicos, seu telos é atingido instantaneamente. Estados, por outro lado, são atélicos, pois seu fim não é determinado lexicalmente.

Para se fazer uma pergunta sobre o tempo de ocorrência de achievements, utiliza-se at what time? 'em que momento?'; enquanto que para se perguntar sobre estados, utiliza-se (for) how long? 'por quanto tempo'. Uma vez que estados e atividades têm em comum o fato

36 No entanto, alguns verbos de achievement podem aparecer no progressivo com leitura de fase preparatória para o evento como em John is dying 'O João está morrendo', que significa que o João está em vias de morrer, e não em processo do evento de morrer.

37 Essa caracterização está longe de ser incontroversa. No entanto, estamos utilizando as divisões tradicionais para termos uma primeira aproximação dos diferentes tipos de predicados em Karitiana.

38 Embora seja relativamente consensual nos trabalhos sobre o assunto considerar que os achievements sejam eventos instantâneos, Piñón (1997) chama a atenção para o fato de que eventos realmente instantâneos não existem. Para uma análise mais aprofundada do comportamento dos achievements, ver Piñón (1997). 
de serem atélicos, a pergunta adequada para atividades é a mesma ((for) how long? Por quanto tempo?'). A pergunta apropriada para verbos de accomplishment é, por sua vez, how long did it take to? 'quanto tempo levou para?'. As sentenças abaixo ilustram as diferenças:

(109) a. At what time did you recognize it?

ACHIEVEMENT

'Em que momento você o reconheceu?'

b. (For) how long did you love her?'

ESTADO

'Por quanto tempo você a amou?'

c. (For) how long did you run?

ATIVIDADE

'Por quanto tempo você correu?'

d. How long did it take to draw the circle?

ACCOMPLISHMENT

'Quanto tempo levou para desenhar o círculo?'

(exemplos de Vendler 1957, p. 145 a 147)

Resumindo, acomplishments e achievements são predicados télicos, ou seja, possuem um telos, um final determinado, enquanto que atividades e estados são predicados atélicos, que não possuem um telos determinado. Entre os predicados télicos, accomplishments são aqueles que possuem uma duração até atingir o telos, enquanto que os achievements são predicados que descrevem eventos pontuais. Entre os predicados atélicos, atividades são os eventos que descrevem um processo em duração, enquanto que estados são predicados estáveis dos indivíduos.

A tabela 7 apresenta resumidamente a proposta de Vendler (1957) associada ao que o autor chama de esquema de tempo. Trata-se basicamente da relação entre os eventos de cada classe com o tempo. 
Tabela 7: Classes Vendlerianas e sua Interação com o Tempo ${ }^{39}$

\begin{tabular}{|c|c|c|}
\hline Classificação & Exemplos & Descrição \\
\hline Atividade & $\begin{array}{l}\text { correr, } \\
\text { empurrar um } \\
\text { carrinho }\end{array}$ & $\begin{array}{l}A \text { was running at time } t \text { ('A estava } \\
\text { correndo no tempo } t^{\prime} \text { ) significa que o } \\
\text { instante de tempo } t \text { está inserido em um } \\
\text { intervalo de tempo em que } A \text { estava } \\
\text { correndo do inicio ao fim. }\end{array}$ \\
\hline Accomplishmet & $\begin{array}{l}\text { desenhar um } \\
\text { círculo, correr } \\
\text { uma milha }\end{array}$ & $\begin{array}{l}A \text { was drawing a circle at } t \text { (' } A \text { estava } \\
\text { desenhando um círculo em } t^{\prime} \text { ) significa } \\
\text { que } t \text { está inserido no intervalo de tempo } \\
\text { em que } A \text { desenhou o circulo. }\end{array}$ \\
\hline Achievement & $\begin{array}{l}\text { alcançar o topo, } \\
\text { parar o avião }\end{array}$ & $\begin{array}{l}A \text { won a race between } t_{1} \text { to } t_{2}(' A \text { ganhou a } \\
\left.\text { corrida entre } t_{1} \text { e } t_{2}{ }^{\prime}\right) \text { significa que o } \\
\text { instante de tempo em que } A \text { ganhou a } \\
\text { corrida está entre } t_{1} \text { e } t_{2} \text {. }\end{array}$ \\
\hline Estado & $\begin{array}{l}\text { amar, } \\
\text { saber }\end{array}$ & $\begin{array}{l}\text { A loved somebody from } t_{1} \text { to } t_{2} \text { ('A amou } \\
\text { alguém entre } t_{1} \text { e } t_{2} \text { ) significa que em } \\
\text { qualquer instante entre } t_{1} \text { e } t_{2} A \text { amou } \\
\text { aquela pessoa. }\end{array}$ \\
\hline
\end{tabular}

O esquema de tempo de Vendler (1957) mostra que os verbos de atividade e estados estão lexicalmente associados a períodos de tempo que não são nem únicos nem determinados. Os instantes de tempo são descritos como em um e qualquer. Accomplishments e achievements, por outro lado, implicam a noção de um tempo único e determinado, descritos com o artigo definido $o$ na tabela acima.

Esta subseção mostrou a proposta clássica de Vendler (1957) para as classes verbais. A próxima subseção discute a ampliação dessa proposta para o sintagma verbal (VP) uma vez que a natureza do objeto direto está associada à natureza do evento descrito verbo.

\footnotetext{
39 Mantivemos as frases em inglês porque Vendler (1957) faz uma proposta baseada nos dados dessa língua. Os destaques do autor com o objetivo de enfatizar se é um ou o intervalo foram mantidos.
} 


\subsubsection{A Acionalidade como uma Propriedade de VPs}

O objetivo desta subseção é discutir os trabalhos de Verkuyl (1996) e Krifka (1998) de ampliação da caracterização da acionalidade para o sintagma verbal ${ }^{40}$. $\mathrm{O}$ argumento principal para se considerar a acionalidade como um fenômeno do VP e não apenas do verbo está no fato de que a natureza do objeto influencia na classificação do evento descrito. $\mathrm{O}$ exemplo abaixo ilustra essa propriedade. O verbo comer descreve um evento télico quando tem um objeto do tipo uma maçã, mas é atélico quando é utilizado com um objeto como maçãs.

a. O João comeu uma maçã.

TÉLICO

b. $\quad$ O João comeu maçãs.

ATÉLICO

O trabalho influente de Verkuyl (1996) critica a proposta de Vendler (1957) afirmando que o autor atribuiu muita importância para o domínio temporal. Verkuyl (1996) defende uma abordagem composicional para a acionalidade que leve em conta a contribuição semântica do DP objeto. $\mathrm{O}$ autor foca sua análise na distinção entre terminativo e durativo. $\mathrm{O}$ terminativo é o que a literatura em geral chama de télico e o durativo, de atélico. Para Verkuyl (1996), a distinção entre aspecto terminativo e durativo é construída estruturalmente com a contribuição de propriedades temporais e atemporais.

As propriedades temporais são traduzidas pelo traço [ \pm ADD TO] que divide os predicados eventivos [+ADD TO] dos predicados estativos [- ADD TO]. As propriedades atemporais dizem respeito à contribuição do DP para o aspecto e estão associdados ao traço [ \pm SQA]. Segundo o autor, se o DP objeto de um verbo tiver uma quantidade especificada ele será [+SQA] e se não tiver uma quantidade especificada será [-SQA]. A relação entre os dois traços deriva as classes acionais principais. Verkuyl (1996) não diferencia accomplishments de achievements e considera todos os predicados télicos como eventos ${ }^{41}$. O autor denomina os verbos de atividade como processos. Do ponto de vista temporal, processos e eventos são [+ADD TO], enquanto que estados são [- ADD TO]. O que diferencia processos de eventos é

40 Embora a afirmação de que a telicidade seja uma característica do VP e não do verbo seja bem aceita nos estudos atuais, Filip e Rothstein (2005) afirmam que essa não é uma propriedade universal das línguas naturais. As autoras defendem que, em línguas eslavas, a telicidade é uma propriedade dos verbos.

${ }^{41}$ O termo se confunde com a noção de eventos utilizadas pela Semântica de Eventos, como em Parsons (1990), mas decidimos manter a terminologia do autor. Para Verkuyl (1996) eventos são, portanto, os predicados télicos. 
o domínio atemporal. Se o predicado tiver um DP [+SQA], ou seja, com quantidade especificada, ele será um evento (predicado télico). Se o predicado tiver um DP [-SQA], com quantidade não especificada, ele será um processo. A tabela 8 abaixo representa a proposta:

Tabela 8: Proposta de Verkuyl (1996) Baseada nos Traços [ \pm ADD TO] e [ \pm SQA]

\begin{tabular}{|c|c|c|c|}
\hline & & \multicolumn{2}{|l|}{ Domínio Temporal } \\
\hline & & $\begin{array}{l}\text { Eventivo } \\
\text { [+ADD TO] }\end{array}$ & $\begin{array}{l}\text { Não eventivo } \\
\text { [-ADD TO] }\end{array}$ \\
\hline \multirow[t]{2}{*}{$\begin{array}{l}\text { Domínio } \\
\text { Atemporal }\end{array}$} & $\begin{array}{l}\text { DP com quantidade indefinida } \\
\text { [-SQA] }\end{array}$ & Processo & \multirow{2}{*}{ Estado } \\
\hline & $\begin{array}{l}\text { DP com quantidade definida } \\
\text { [+SQA] }\end{array}$ & $\begin{array}{l}\text { Evento } \\
\text { (Predicado Télico) }\end{array}$ & \\
\hline
\end{tabular}

Segundo a proposta de Verkuyl (1996), um predicado só será terminativo (télico) se tiver os dois traços marcadas com positivo. Ou seja, o predicado tem que ser eventivo, por oposição a estativo, e deve ter um argumento com quantidade especificada. As sentenças abaixo ilustram as quatro possibilidades derivadas das associações com os traços.

$\begin{array}{cll}\text { (111) a. } & \text { John wanted sandwichs. } & \text { [-ADD TO }]+[-\mathbf{S Q A}]=[- \text { Termin. }] \\ & \text { 'O John quis sanduíches' } & \text { ESTADO } \\ \text { b. } & \text { John wanted a sandwich. } & \text { [-ADD TO }]+[+ \text { SQA }]=[- \text { Termin. }] \\ & \text { 'O John quis um sanduíche' } & \text { ESTADO } \\ \text { c. } & \text { John ate sandwichs. } & \text { [+ADD TO }]+[-\mathbf{S Q A}]=[- \text { Termin. }] \\ & \text { 'O John comeu sanduíches' } & \text { PROCESSO } \\ \text { d. } & \text { John ate a sandwich. } & \text { [+ADD TO }]+[+ \text { SQA }]=[+ \text { Termin. }] \\ & \text { 'O John comeu um sanduíche' } & \text { EVENTO }\end{array}$

(exemplos baseados em Verkuyl 1996, pag. 22)

A proposta de Verkuyl (1996), embora tenha sido bastante influente por trazer para a discussão da acionalidade a contribuição do DP, sofreu muitas críticas. Uma delas diz respeito, por exemplo a predicados de atividade (processos) com DPs definidos, como no exemplo abaixo. Segundo a proposta de Verkuyl (1996) baseada nos traços, o evento descrito 
em (112) deveria ser terminativo (télico), uma vez que trata-se de um predicado eventivo ([+ADD TO]) e com DP com quantidade definida ([+SQA]). No entanto, não é esse o caso; trata-se de uma atividade, um evento atélico ${ }^{42}$.

\section{O João empurrou o carro.}

Krifka (1998) propõe uma abordagem mereológica para a telicidade e resolve problemas como o da sentença (112) acima. A mereologia é uma teoria de relações de partetodo que ficou célebre a partir da análise da distinção contável-massivo dos sintagmas nominais baseada na estrutura algébrica de semirreticulado proposta por Link $(1983)^{43}$. No reticulado do domínio contável, o conjunto dos átomos ( $a, b$ e $c$ no exemplo abaixo) representa a denotação do nome contável singular, enquanto que o plural é representado pelo reticulado inteiro. A estrutura abaixo, representa um modelo de reticulado.

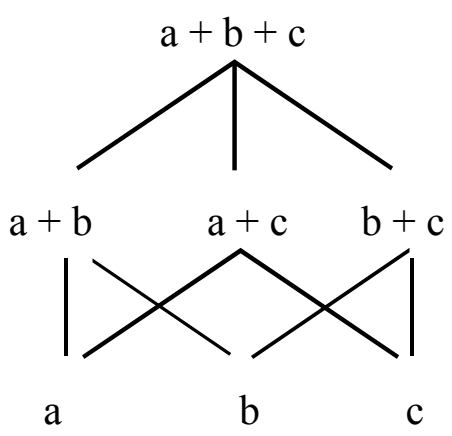

Para Krifka (1998), a telicidade pode ser definida em termos mereológicos. Mais especificamente, ela é discutida em termos das propriedades semânticas de cumulatividade e quantização. Essas propriedades são apresentadas respectivamente em (114) e (115):

42 Para mais críticas à proposta de Verkuyl (1996), ver Krifka (1996).

43 Link (1983) utiliza uma estrutura tecnicamente conhecida como semirreticulado superior (do inglês join semi-lattice) porque não considera os elementos ínfimos, mas apenas os supremos. Para mais detalhes, ver Davey e Priestley (1990). 
Cumulatividade:

$\forall \mathrm{X} \subseteq \mathrm{U}_{\mathrm{P}}\left[\mathrm{CUM}_{\mathrm{P}}(\mathrm{X}) \leftrightarrow \exists \mathrm{x}, \mathrm{y}[\mathrm{X}(\mathrm{x}) \wedge \mathrm{X}(\mathrm{y}) \wedge \neg \mathrm{x}=\mathrm{y}] \wedge \forall \mathrm{x}, \mathrm{y}[\mathrm{X}(\mathrm{x}) \wedge \mathrm{X}(\mathrm{y}) \rightarrow\right.$ $\mathrm{X}(\mathrm{x} \oplus \mathrm{P} \mathrm{y})]]$

Um predicado $\mathrm{X}$ é cumulativo se, e somente se, se existem dois argumentos diferentes $\mathrm{x}$ e y aos quais o predicado X se aplica individualmente, então o predicado $\mathrm{X}$ também se aplica à soma de $\mathrm{x}$ e $\mathrm{y}$.

(definição 3 de Krifka 1998, p. 3)

(115) Quantização:

$\forall \mathrm{X} \subseteq \mathrm{U}_{\mathrm{P}}\left[\mathrm{QUA} A_{\mathrm{P}}(\mathrm{X}) \leftrightarrow \forall \mathrm{x}, \mathrm{y}[\mathrm{X}(\mathrm{x}) \wedge \mathrm{X}(\mathrm{y}) \rightarrow \neg \mathrm{y}<\mathrm{p} \mathrm{x}]\right]$

Um predicado $\mathrm{X}$ é quantizado se, e somente se, sempre que ele se aplica a dois argumentos $\mathrm{x}$ e $\mathrm{y}$, y nunca é uma parte de $\mathrm{x}$.

(definição 4 de Krifka 1998, p. 3)

Sintagmas nominais massivos como água e plurais como maçãs são cumulativos. Se o predicado água se aplica a duas porções diferentes de água, ele também se aplica à soma dessas porções, que continua sendo uma porção de água. Se o predicado maçãs se aplica a dois conjuntos distintos de maçãs, ele também se aplica à soma desses conjuntos, que continua sendo um conjunto de maçãs. Água e maçãs não são predicados quantizados. Se uma porção de água é dividida, o predicado água continua se aplicando às partes. O mesmo ocorre com maçãs. Porções menores de maçãs continuam sendo maçãs. Um sintagma como três maçãs, por outro lado, é um predicado quantizado. Isso porque o predicado três maçãs não se aplica a partes de três maçãs: a uma parte do conjunto de três maçãs contendo, por exemplo, duas maçãs, não se pode aplicar o predicado três maçãs. Ademais, três maçãs não é um predicado cumulativo. Se o predicado três maçãs se aplica a dois conjuntos distintos, ele não se aplica a soma desses dois conjuntos, que configuram juntos seis maçãs.

Além das propriedades de cumulatividade e quantização, a proposta de Krifka (1998) para a telicidade está associada às noções de parte inicial e final dos eventos. De modo geral, uma parte inicial de um evento é sua parte que não possui nenhuma outra parte que a preceda e a parte final é aquela que não possui nenhuma outra parte que a suceda. As definições estão representas abaixo. 
a.

$\forall \mathrm{e}, \mathrm{e}^{\prime} \in \mathrm{U}_{\mathrm{E}}\left[\mathrm{INI}_{\mathrm{E}}\left(\mathrm{e}^{\prime}, \mathrm{e}\right) \leftrightarrow \mathrm{e}^{\prime} \leq_{\mathrm{D}} \mathrm{e} \wedge \neg \exists \mathrm{e}^{\prime \prime} \in \mathrm{U}_{\mathrm{E}}\left[\mathrm{e}^{\prime \prime} \leq_{\mathrm{E}} \mathrm{e} \wedge \mathrm{e}^{\prime \prime} \ll_{\mathrm{E}} \mathrm{e}^{\prime}\right]\right]$

Um evento $e^{\prime}$ é uma parte inicial de um evento $e$, se, e somente se, $e^{\prime}$ é uma parte de $e$ e não existe nenhum evento $e^{\prime \prime}$ que seja também parte de $e$ e que preceda $e^{\prime}$.

b. $\quad \forall \mathrm{e}, \mathrm{e}^{\prime} \in \mathrm{U}_{\mathrm{E}}\left[\mathrm{FIN}_{\mathrm{E}}\left(\mathrm{e}^{\prime}, \mathrm{e}\right) \leftrightarrow \mathrm{e}^{\prime} \leq_{\mathrm{D}} \mathrm{e} \wedge \neg \exists \mathrm{e}^{\prime \prime} \in \mathrm{U}_{\mathrm{E}}\left[\mathrm{e}^{\prime \prime} \leq_{\mathrm{E}} \mathrm{e} \wedge \mathrm{e}^{\prime} «_{\mathrm{E}} \mathrm{e}^{\prime \prime}\right]\right]$

Um evento $e^{\prime}$ é uma parte final de um evento $e$, se e somente, se; $e^{\prime}$ é uma parte de $e$ e não existe nenhum evento $e^{\prime \prime}$ que seja também parte de $e$ e que suceda $e^{\prime}$.

(definições 36a e 36b de Krifka 1998, p. 9)

Utilizando as noções de parte inicial e final de um evento, Krifka (1998) afirma que a telicidade pode ser definida como uma propriedade de um predicado $\mathbf{X}$ que se aplica a um evento de tal forma que todas as partes desse evento são partes iniciais e finais dele. A definição de telicidade está descrita em (117):

$\forall \mathrm{X} \subseteq \mathrm{U}_{\mathrm{E}}\left[\mathrm{TEL}_{\mathrm{E}}(\mathrm{X}) \leftrightarrow \forall \mathrm{e}, \mathrm{e}^{\prime} \in \mathrm{U}_{\mathrm{E}}\left[\mathrm{X}(\mathrm{e}) \wedge \mathrm{X}(\mathrm{e}) \wedge \mathrm{e}^{\prime} \leq_{\mathrm{E}} \mathrm{e} \rightarrow \operatorname{INI}_{\mathrm{E}}\left(\mathrm{e}^{\prime}, \mathrm{e}\right) \wedge \mathrm{FIN}_{\mathrm{E}}\left(\mathrm{e}^{\prime}, \mathrm{e}\right)\right]\right]$

Um predicado $\mathrm{X}$ é télico se, e somente se, para todo evento $e$ e para todo evento $e^{\prime}$ aos quais o predicado X se aplica, se $e^{\prime}$ é parte de $e$, então $e^{\prime}$ é parte inicial e parte final de $e$.

(definição 37 de Krifka 1998, p. 9)

Segundo a definição em (117), os predicados télicos são quantizados, uma vez que não há nenhuma parte do evento e que seja diferente de e a que se possa atribuir o predicado $\mathbf{X}$. Os predicados cumulativos por outro lado, são atélicos. Se um predicado cumulativo $\mathbf{X}$ se aplica a pelo menos dois eventos e e e' que não são contemporâneos, ou seja, se é possível encontrar um e" que seja parte de e e que suceda e'; então, $\mathbf{X}$ é um predicado atélico.

Para explicar a diferença entre predicados télicos como comer três maçãs e predicados atélicos como comer maçãs, Krifka (1998) assume que existe um homorfismo entre os eventos e seus argumentos semânticos que relaciona suas estruturas reticuladas. Segundo o autor, as estruturas algébricas de ambos os domínios estão associadas por relações temáticas. Dessa forma, argumentos quantizados como três maçãs estão associados a eventos 
quantizados e, portanto, télicos; enquanto que argumentos cumulativos, como maçãs, estão relacionados a eventos cumulativos, e portanto, atélicos.

a. $\quad$ O João comeu uma maçã.

TÉLICO

b. O João comeu maçãs.

ATÉLICO

É importante notar, no entanto, que essa relação homomórfica entre os domínios dos indivíduos denotados pelos argumentos e dos eventos denotados pelos verbos se aplica apenas a predicados que são incrementais. Predicados incrementais são aqueles em que o objeto é construído ou consumido durante o processo do evento. Desse modo; comer, fazer, e destruir são exemplos de predicados incrementais. Mas, empurrar, por outro lado, não é incremental. Isso quer dizer que não há uma relação homomórfica entre o evento de empurrar e o objeto empurrado. É por isso que, embora no exemplo abaixo o objeto seja quantizado, o evento descrito é atélico.

O João empurrou o carro.

Esta subseção mostrou as proposta de Verkuyl (1996) e Krifka (1998) de expansão para o VP da classificação dos predicados verbais em classes acionais. A contribuição de Krifka (1998) à proposta de Verkuyl (1996) é atribuir uma relação homomórfica entre a natureza do evento e a propriedade dos DPs somente a predicados incrementais. A próxima seção apresenta as críticas de Rothstein (2004) à proposta de Krifka (1998) e apresenta uma abordagem para a telicidade baseada em atomicidade.

\subsubsection{Rothstein $(2004,2008)$}

Rothstein (2004, 2008) critica a proposta de Krifka (1998) apresentada acima, mostrando que uma abordagem para a telicidade baseada na quantização revela problemas. No exemplo (120), abaixo, por exemplo, o predicado denota um evento télico, embora o argumento seja cumulativo. Uma sequência de números é um predicado cumulativo porque uma sequência de números somada a uma sequência de números resulta em uma nova sequência de números. Segundo a proposta de Krifka (1998), que assume um homomorfismo 
entre o domínio dos eventos e o domínio dos indivíduos, o predicado verbal deveria ser cumulativo, como seu objeto e, portanto, atélico.

João escreveu uma sequencia de números em um minuto.

TÉLICO

(traduzido do exemplo 13a de Rothstein 2008, p.8)

Para Rothstein (2004), a telicidade é uma expressão da atomicidade. Sua proposta geral é a de que todo o domínio verbal é gramaticalmente contável. Essa análise será explorada em detalhes na próxima seção deste capítulo. Segundo a autora, os verbos denotam expressões do tipo:

$\llbracket \mathrm{V} \rrbracket=\lambda \mathrm{e} . \mathrm{P}(\mathrm{e}) \& \operatorname{MEAS}(\mathrm{e})=<1, \mathrm{U}>$

(representação 21 de Rothstein 2008, p.13)

Na representação acima, MEAS é uma função de medida que gera pares ordenados do tipo $<$ número natural, unidade de medida $>$. Segundo a proposta em (121), a denotação de um verbo é um predicado de eventos tal que a medida do evento é dada segundo a unidade $\mathbf{U}$. Para Rothstein $(2004,2008)$ a diferença entre VPs télicos e atélicos está nos critérios de individuação de um evento. Quando os critérios de individuação (U) são lexicalmente especificados, o evento é telico, e quando não são especificados o evento é atélico. Isso está de acordo com a intuição de que o que conta como um átomo de um evento de reconhecer algo ou de desenhar um círculo faz parte da denotação desses predicados; enquanto que o que conta como um evento completo de correr ou de saber depende do contexto. Os exemplos abaixo ilustram essa diferença.

$$
\begin{aligned}
& \text { a. } \quad \llbracket \text { reconhecer } \rrbracket=\lambda \text { e. } \mathrm{P}(\mathrm{e}) \wedge \operatorname{MEAS}(\mathrm{e})=<1 \text {,reconhecida }> \\
& \text { b. } \llbracket \text { correr } \rrbracket=\lambda \mathrm{e} . \mathrm{P}(\mathrm{e}) \wedge \operatorname{MEAS}(\mathrm{e})=<1, \mathrm{U}>
\end{aligned}
$$

Nesse sentido, Rothstein $(2004,2008)$ afirma que os predicados télicos são naturalmente atômicos. Segundo essa proposta, verbos de atividade e estado são inerentemente não atômicos, enquanto que os verbos de achievement são atômicos. Verbos de accomplishment são considerados potencialmente atômicos, a depender da natureza de seu 
objeto.

Além de propor que a telicidade é derivada da atomicidade, Rothstein $(2004,2008)$ reanalisa as distinções propostas em Vendler (1957) segundo dois traços básicos: (i) se eles são passíveis de se estender temporalmente e, (ii) se envolvem evento de mudança.

O traço de mudança é o de telicidade e é o que separa accomplishments e achievements de um lado e estados e atividades de outro. Já o traço de duração separa por um lado as atividades e accomplishments como eventualidades que são durativas, têm estágios em sua estrutura interna e, por outro, os estados e achievements que não têm. Os achievements expressam mudanças de duração mínima (eventos instantâneos), enquanto que os estados não têm estágios discerníveis em sua denotação.

Tabela 9: Características de Rothstein $(2004,2008)$ para as Classes Vendlerianas

\begin{tabular}{|l|c|c|}
\hline \multicolumn{1}{|c|}{ Classes } & $\begin{array}{c}\text { Eventos mínimos são } \\
\text { estendíveis }\end{array}$ & Evento de mudança \\
\hline Estados & - & - \\
\hline Atividades & + & - \\
\hline Achievements & - & + \\
\hline Accomplishments & + & + \\
\hline
\end{tabular}

Esta subseção fecha a apresentação de propostas para a divisão dos predicados verbais em accomplishments, achievements, atividades e estados. A próxima seção discute uma divisão dos estativos que é bastante utilizada na literatura: a divisão entre predicados individual-level e stage-level.

\subsubsection{Estativos stage-level e individual level}

O objetivo desta seção é apresentar uma outra distinção importante dos predicados verbais discutida na literatura. Tradicionalmente, a literatura semântica divide os predicados estativos em predicados stage-level e predicados individual-level. Carlson (1980) apresenta uma distinção entre propriedades transitórias dos indivíduos e susceptíveis a repetições propriedades stage-level - e propriedades que são consideradas permanentes - propriedades individual-level. Essas propriedades são expressas, respectivamente, por predicados stagelevel e individual-level. São predicados stage-level, por exemplo, estar feliz, querer, estar 
disponível, etc. Exemplos de predicados individual-level são acreditar em Deus, amar, saber, conhecer, ser brasileiro. Muitos trabalhos têm mostrado que essa diferença é relevante para o estudo de vários fenômenos gramaticais - como por exemplo, o comportamento dos plurais nus do inglês, descrito em Carlson (1980). Carson (1980) partiu do fato de que os plurais nus do inglês podiam ter leitura genérica ou existencial dependendo do tipo de predicado verbal. Em sentenças com predicados individual-level, como (123a), o plural têm leitura genérica; enquanto em sentenças com predicado stage-level, como em (123b), o plural tem leitura existencial.
a. Dogs are intelligent.
LEITURA GENÉRICA
'Cachorros são inteligentes'
b. Dogs are sick.
LEITURA EXISTENCIAL
'Cachorros estão doentes ${ }^{44 !}$

(exemplos de Carlson 1980, p. 451)

Kratzer (1995) explora essa diferença e propõe que predicados estativos stage-level e individual-level têm estruturas argumentais diferentes. Predicados stage-level têm comportamento semelhante ao dos outros predicados verbais e possuem um argumento davidsoniano de evento $^{45}$. Predicados individual-level, por outro lado, não possuem esse argumento. A autora sustenta a sua proposta com base nos dados com o locativo em alemão, sentenças introduzidas por when 'quando' e com plurais nus do inglês. Não vamos apresentar todos os detalhes da argumentação, mas o que se pode dizer é que considerar que os predicados stage-level, mas não os individual-level, têm um argumento extra de evento traz consequências bastante desejáveis para a análise de muitas construções em que ocorrem. Vamos focar a discussão no argumento que é mais relevante para esta tese que é o que diz respeito à modificação de predicados verbais.

O primeiro capítulo mostrou que a modificação de predicados verbais é um dos argumentos que Parsons (1990) apresenta como evidência a favor de uma Semântica de Eventos. Modificadores verbais típicos são predicados de eventos. Logo, para que a modificação ocorra, é preciso que haja um argumento evento sobre o qual se possa predicar. O

\footnotetext{
44 Optamos por manter um plural nu na tradução em português, embora a sentença não pareça natural.

45 A proposta para uma Semântica de Eventos baseada em Davidson (1967) e Parsons (1990) foi apresentada no capítulo 1 .
} 
que se espera a partir da proposta de Kratzer (1995) de que predicados individual-level não possuem um argumento evento é, portanto, que esses predicados não possam ser modificados. A expectativa é confirmada pelos dados, como se pode ver em (124) abaixo (exemplos adaptados de Kratzer 1995). As sentenças (124a) e (124b) mostram que predicados stagelevel como dançar podem ser modificados por locuções adverbiais de lugar como no pátio e de tempo, como hoje. O predicado é uma dançarina, por outro lado, é um predicado individual-level e, portanto, não pode ser modificado, como mostram os dados (124c) e $(124 d)^{46}$.

(124) a. Maria está dançando no pátio.

b. Maria está dançando hoje.

c. \# Maria é uma dançarina no pátio.

d. \# Maria é uma dançarina hoje.

Kratzer (1995) chama a atenção para o fato de que essa distinção não é tão categórica a ponto de ser lexical. Não é raro um predicado individual-level ser interpretado como stagelevel. Por exemplo, ter cabelos castanhos é canonicamente um predicado individual-level; mas, ele pode ser considerado como uma propriedade bastante transitória para um sujeito que frequentemente tinge os cabelos de cores diferentes. Nos casos em que isso ocorre, o predicado passa a ter a estrutura argumental de um predicado stage-level. O mesmo ocorre com possíveis sentenças com predicados individual-level modificados. A sentença (124d) acima, por exemplo, pode ser considerada uma sentença adequada em um contexto em que o predicado ser uma dançarina é interpretado como um predicado stage-level, como 'se passar por uma bailarina'.

Esta subseção fechou a seção sobre as teorias sobre as classes verbais. A proposta de dividir os predicados verbais em estados, atividades, achievements e accomplishments não é totalmente incontroversa, mas foi a que ganhou mais atenção na literatura sobre acionalidade e pode ser considerada como uma primeira classificação dos predicados verbais de uma língua. A próxima subseção discute a pertinência dessa classificação em Karitiana.

\footnotetext{
46 Na proposta de Kratzer (1995), a distinção entre predicados stage-level e individual-level não se aplica apenas a predicados estativos. Predicados individual-level são tipicamente estativos, mas stage-levels podem ser não estativos.
} 


\subsection{Classes Verbais em Karitiana}

O foco desta subseção é discutir a classificação dos predicados verbais em Karitiana segundo a sua acionalidade. Seu principal objetivo é discutir a pertinência de se classificar os predicados verbais da língua em estados, atividades, achievements e accomplishments. Ou seja, pretende-se verificar se, em Karitiana, são encotradas as mesmas classes acionais que se discute na literaura para o inglês e outras línguas. Essa discussão é relevante porque essa distinção será utilizada para a análise da modificação de grau no domínio verbal na língua. Para tal, vamos, primeiramente, discutir a relação entre a acionalidade e a natureza dos sintagmas nominais na língua. Conforme apresentado no capítulo 1 , os nomes comuns em Karitiana são sempre nus e têm sempre número neutro. A subseção 1.3.1 explora quais as consequências dessa característica para as classes acionais na língua. Em seguida, a seção 1.3.2 apresenta uma seleção de verbos separados segundo sua acionalidade. Foram escolhidos os verbos que são usualmente classificados em cada classe, levando-se em conta a caracterítica particular dos NPs em Karitiana. Na subseção 1.3.3, esses verbos são submetidos a uma variedade de testes que foram tradicionalmente utilizados para o diagnóstico das classes verbais em muitas línguas. Seu objetivo central é discutir se esses testes são adequados em Karitiana e se algum deles pode ser utilizado com segurança para distinguir as classes na língua.

\subsubsection{Os Sintagmas Nominais e as Classes Acionais em Karitiana}

O objetivo desta subeção é discutir quais as consequências da natureza particular dos sintagmas nominais com nomes comuns em Karitiana para a classificação dos predicados verbais segundo a acionalidade. Conforme apresentado no capítulo 1, os sintagmas nominais em Karitiana não apresentam material funcional de nenhum tipo. A língua não possui determinantes, quantificadores ou classificadores. Além disso, em Karitiana, não há marcas para número no domínio nominal. Os sintagmas nominais formados por nomes comuns não modificados por expressões de quantidade têm uma denotação neutra para número, ou seja, denotam indivíduos singulares e plurais. A sentença (45), repetida abaixo em (125) ilustra essa propriedade. Nomes comuns que denotam indivíduos, como jonso 'mulher', ou que denotam massa, como ese 'água', que se comportam respectivamente como nomes contáveis e massivos 
nas línguas descritas na literatura, aprecem nus na sentença e denotam quantidades indefinidas, ou seja, a sentença pode ser utilizada em contextos em que há uma ou mais mulheres e uma quantidade indefinida de água.

Jonso Ø-naka-ot-Ø

$=(45)=(93)$

mulher 3-DECL-buscar-NFUT água

'Mulher buscou água'

Uma vez que os sintagmas nominais possuem denotação neutra para número, eles possuem a propriedade da cumulatividade. Conforme apresentado acima, na seção 1.2.2, a cumulatividade é uma das propriedades que Krifka (1998) utiliza para sua proposta de telicidade. A definição de cumulatividade é retomada em (126).

(126) Cumulatividade:

$\forall \mathrm{X} \subseteq \mathrm{U}_{\mathrm{P}}\left[\mathrm{CUM}_{\mathrm{P}}(\mathrm{X}) \leftrightarrow \exists \mathrm{x}, \mathrm{y}[\mathrm{X}(\mathrm{x}) \wedge \mathrm{X}(\mathrm{y}) \wedge \neg \mathrm{x}=\mathrm{y}] \wedge \forall \mathrm{x}, \mathrm{y}[\mathrm{X}(\mathrm{x}) \wedge \mathrm{X}(\mathrm{y}) \rightarrow\right.$ $\mathrm{X}(\mathrm{x} \oplus \mathrm{P} \mathrm{y})]]$

Um predicado $\mathrm{X}$ é cumulativo se, e somente se, se existem dois argumentos diferentes $\mathrm{x}$ e $\mathrm{y}$ aos quais o predicado X se aplica, então o predicado x também se aplica à soma de x e y.

(definição 3 de Krifka 1998, p. 3)

Em Karitiana, um nome como jonso 'mulher' denota átomos e somas. Ele é, portanto, cumulativo. Uma representação da denotação de jonso 'mulher' baseada em conjuntos é apresentada abaixo, em (127), para um contexto em que existem apenas três mulheres. Como se pode observar, se o predicado jonso se aplica, por exemplo, a mulher ${ }_{1}$ e se aplica a mulher $_{2}$, ele se aplica também à soma mulher $_{1} \oplus$ mulher $_{2}$.

(127) $\llbracket$ jonso $\rrbracket=\left\{\right.$ mulher $_{1}$, mulher $_{2}$, mulher $_{3}$, mulher $_{1} \oplus$ mulher $_{2}$, mulher $_{1} \oplus$ mulher $_{3}$, mulher $_{2} \oplus$ mulher $_{3}$, mulher $_{1} \oplus$ mulher $_{2} \oplus$ mulher $_{3}$ \} 
$\mathrm{O}$ fato de os sintagmas nominais formados por nomes comuns em Karitiana serem cumulativos traz consequências para a telicidade na língua. Conforme aprensentado na seção 1.2.2, a classificação dos predicados verbais segundo a sua acionalidade é uma caracteristica dos VPs, e não do verbo apenas. Verkuyl (1996) e Krifka (1998) mostraram que a natureza dos sintagmas que ocupam a posição de objeto de um predicado interfere na sua classe acional, especialmente no caso dos predicados de accomplishment, como se pode ver nos exemplos abaixo:
a. $\quad$ O João comeu uma maçã.
DP QUANTIZADO $=$ PREDICADO TÉLICO
b. O João comeu maçãs.
DP CUMULATIVO $=$ PREDICADO ATÉLICO

Uma vez que em Karitiana os sintagmas nominais nus possuem denotação cumulativa, os verbos incrementais do tipo comer e construir vão ser sempre atélicos com esses sintagmas como argumentos, como mostram as sentenças abaixo.

João Ø-naka-'y-t kinda'o.

João 3-DECL-comer-NFUT fruta

'João comeu fruta (uma ou mais frutas)'

EVENTO ATÉLICO

(130) Ivan i-kokot- $\varnothing \quad$ pa-ty.

Ivan PART-passar-ABS rua-OBL

'O Ivan atravessou rua (uma ou mais ruas)'

EVENTO ATÉLICO

As poucas estratégias para se obter um argumento nominal quantizado em Karitiana são nomes próprios ou argumentos modificados por numerais ${ }^{47}$. Os exemplos em (131) e (132) mostram como é possível telicizar os eventos descritos acima em (129) e (130) por meio dessas estratégias.
João Ø-naka-'y-t
myhin-t kinda'o.
João 3-DECL-comer-NFUT um-OBL fruta
'O João comeu uma fruta'

EVENTO TÉLICO

${ }_{47}$ No capítulo 1, os numerais em Karitiana foram descritos como adjuntos do NP. 

(132) Ivan i-kokot- $\varnothing \quad$ Sete de Setembro dewota kyyn.
Ivan PART-passar-ABS Sete de setembro outro.lado para
'O Ivan atravessou a rua Sete de Setembro'

EVENTO TÉLICO

Além disso, alguns sintagmas, embora não sejam gramaticalmente quantizados, têm um leitura de nome próprio na comunidade. Esse é o caso, por exemplo do sintagma pyejepa que quer dizer 'escola'. Na aldeia há apenas uma escola, logo, no contexto, o sintagma tem apenas interpretação definida. Quando se diz pyejepa, é o mesmo que se dizer o nome da escola. Uma forma de salientar essa propriedade é colocando o adjetivo goot 'novo'. Assim, o sintagma pyejepa goot denota diretamente o prédio novo que foi construído para abrigar a escola da aldeia. O exemplo abaixo apresenta uma sentença com esse sintagma. O evento descrito em (133) é considerado télico porque, no contexto de uso, o argumento do verbo só pode ter interpretação definida.

João Ø-naka-pog-<a>-t pyejepa goot.

João 3-DECL-pintar.de.branco-NFUT escola novo

'O João pintou a escola nova de branco'

Os predicados de achievement também têm uma classificação dependente da natureza dos sintagmas nominais. Como se pode ver no exemplo abaixo, em português, um argumento quantizado faz do predicado encontrar um evento télico, um achievement; enquanto que o verbo com um argumento cumulativo é um predicado de atividade.
a. O João encontrou uma pulga.
EVENTO TÉLICO
b. O João encontrou pulgas.
EVENTO ATÉLICO

Em Karitiana, o verbo typ 'encontrar' também tem uma interpretação atélica quando utilizado com um sintagma nominal com nome comum.
João Ø-naka-typ-Ø
gooj sara'it.
João 3-DECL-encontrar-NFUT carro problema
'O João encontrou problema de carro'

EVENTO ATÉLICO 
Do mesmo modo, o sintagma deso ohyn 'topo de montanha' não é quantizado e denota um ou mais topos de montanhas. Assim, uma vez que os nomes comuns são neutros quanto a uma interpretação definida ou indefinida, uma tradução ao pé da letra para a sentença abaixo seria 'O João chegou em topo de montanha', com um verbo de atividade.

$$
\begin{array}{lll}
\text { João i-otam- } \varnothing \quad \text { deso } & \text { ohyn } & \text { sok. } \\
\text { João PART-chegar-ABS montanha topo } & \text { em } \\
\text { 'O João chegou em topo de montanha' }
\end{array}
$$

Mais uma vez, a saída para se obter um predicado télico será com o uso de um nome próprio ou um numeral. A sentença abaixo descreve uma situação baseada em uma história verídica da comunidade. Antônio Bigode é um índio Karitiana que estava desaparecido há muitos anos. Em 2013, porém, foi encontrado. A sentença abaixo, com o nome próprio, descreve um evento pontual, portanto, um achievement.

$$
\begin{aligned}
& \text { João Ø-naka-typ-Ø Antônio Bigode. } \\
& \text { João 3-DECL-encontrar-NFUT Antônio Bigode } \\
& \text { 'O João encontrou o Antônio Bigode' }
\end{aligned}
$$

EVENTO TÉLICO

Já a sentença abaixo, com um argumento modificado, também descreve um achievement.

$\begin{array}{llll}\text { (138) João i-otam- } \varnothing \text { myhin-t deso } & \text { ohyn } & \text { sok. } \\ \text { João PART-chegar-ABS um-OBL montanha topo } & \text { em } \\ \text { 'O João chegou no topo de uma montanha' } & \end{array}$

EVENTO TÉLICO

Esta subseção problematizou a classificação dos verbos em Karitiana quanto a sua acionalidade considerando a natureza dos sintagmas nominais na língua. Os argumentos dos verbos quando são formados com substantivos comuns não modificados por numerais são sempre cumulativos e formam predicados atélicos. Uma estratégia para se conseguir formar predicados télicos com verbos de accomplishment e achievement é por meio de nomes próprios e sintagmas nominais modificados por numerais. Essas estratégias foram utilizadas 
para a criação de uma tabela de verbos que foi submetida aos testes tradicionais utilizados para a classificação das classes acionais. As próximas subseções apresentam essa tabela e os testes.

\subsubsection{Tabela de Verbos}

Para se aplicar os testes, foi montada uma tabela de verbos com uma classificação prévia dos predicados. A tabela foi montada considerando-se verbos que se comportam nas línguas conhecidas como pertencentes a cada classe, e levando-se em conta a problematização apresentada na seção acima acerca da relação entre telicidade e a natureza dos sintagmas nominais em Karitiana. Desse modo, os predicados de accomplishment e achievement utilizados têm argumentos quantizados como sintagmas nominais modificados por numerais e nomes próprios.

As tabelas abaixo mostram os tipos de predicados selecionados acompanhados de um exemplo simples na língua.

Tabela 10: Predicados de Atividade em Karitiana

\begin{tabular}{|l|l|l|}
\hline \multicolumn{1}{|c|}{ Verbo } & \multicolumn{1}{|c|}{ Tradução } & \multicolumn{1}{c|}{ Exemplo } \\
\hline pykyn & correr & $\begin{array}{l}\text { João i-pykyn-<a>-t. } \\
\text { João PART-correr-ABS } \\
\text { 'O João correu' }\end{array}$ \\
\hline pytim'adn & trabalhar & $\begin{array}{l}\text { João i-pytim'adn-<a>-t. } \\
\text { João PART-trabalhar-ABS } \\
\text { 'O João trabalhou' }\end{array}$ \\
\hline pimbik gooj & empurrar carro & $\begin{array}{l}\text { João Ø-na-pimbik-Ø } \\
\text { João 3-DECL-empurrar-NFUT carro } \\
\text { 'O João empurrou carro' }\end{array}$ \\
\hline tagngã & dirigir carro & $\begin{array}{l}\text { João Ø-naka-tagngã-t gooj. } \\
\text { João 3-DECL-dirigir-NFUT carro } \\
\text { 'O João dirigiu carro' }\end{array}$ \\
\hline
\end{tabular}


Tabela 11: Predicados Estativos Stage-level em Karitiana

\begin{tabular}{|c|c|c|}
\hline Verbo & Tradução & Exemplo \\
\hline siki'y & $\begin{array}{l}\text { querer / } \\
\text { estar com vontade }\end{array}$ & $\begin{array}{l}\text { João i-siki'y-t ese-ty. } \\
\text { João PART-querer-ABS água-OBL } \\
\text { 'O João quer água' }\end{array}$ \\
\hline osedn & estar alegre & $\begin{array}{l}\text { João i-osedn- } \emptyset \text {. } \\
\text { João PART-estar.alegre-ABS } \\
\text { 'O João está alegre' }\end{array}$ \\
\hline em & estar sujo & $\begin{array}{l}\text { João i-em-Ø. } \\
\text { João PART-estar.sujo-ABS } \\
\text { 'O João está sujo' }\end{array}$ \\
\hline pasadn & gostar & $\begin{array}{l}\text { João i-pasadn-Ø Milena-ty. } \\
\text { João PART-gostar-ABS Milena-OBL } \\
\text { 'O João gosta da Milena' }\end{array}$ \\
\hline
\end{tabular}

Tabela 12: Predicados Estativos Individual-level em Karitiana

\begin{tabular}{|c|c|c|}
\hline Verbo & Tradução & Exemplo \\
\hline aka Karitiana & ser Karitiana & $\begin{array}{l}\text { João Ø-na-aka-t } \quad \text { Karitiana-t. } \\
\text { João 3-DECL-COP-NFUT Karitiana-ABS } \\
\text { 'O João é Karitiana' }\end{array}$ \\
\hline $\begin{array}{l}\text { pypyyt } \\
\text { Matematicaty }\end{array}$ & saber Matemática & $\begin{array}{l}\text { João i-pypyyt- } \varnothing \quad \text { Matematica-ty. } \\
\text { João PART-saber-ABS Matemática-OBL } \\
\text { 'O João sabe Matemática' }\end{array}$ \\
\hline $\begin{array}{l}\text { kywytidn } \\
\text { Botỹjty }\end{array}$ & acreditar em Deus & $\begin{array}{l}\text { João i-kywytidn-<a>-t Botỹj-ty. } \\
\text { João PART-acreditar-ABS Deus-OBL } \\
\text { 'O João acredita em Deus' }\end{array}$ \\
\hline
\end{tabular}


Tabela 13: Predicados de Accomplishment em Karitiana

\begin{tabular}{|c|c|c|}
\hline Verbo & Tradução & Exemplo \\
\hline kokot Sete de & atravessar a & Sete de Setembro dewota kyyn. \\
\hline Setembro & Sete de & João PART-passar-ABS Sete de Setembro outro.lado para \\
\hline dewota kyyn & Setembro & 'O João atravessou a rua Sete de Setembro' \\
\hline pog pyejepa & pintar a escola & João Ø-naka-pog-<a>-t pyejepa goot. \\
\hline goot & nova de & João 3-DECL-pintar.de.branco-NFUT escola novo \\
\hline & branco & 'O João pintou a escola nova de branco' \\
\hline Porto & ir para Porto & Porto Velho pip. \\
\hline Velho pip ${ }^{48}$ & Velho & João PART-ir-ABS Porto Velho para \\
\hline & & 'O João foi para Porto Velho' \\
\hline 'a myhint & fazer um colar & João Ø-naka-m-'a-t $\quad$ myhin-t boet. \\
\hline boet & & João 3-DECL-CAUS-fazer-NFUT um-OBL colar \\
\hline & & 'O João fez um colar' \\
\hline
\end{tabular}

Tabela 14: Predicados de Achievement em Karitiana

\begin{tabular}{|c|c|c|}
\hline Verbo & Tradução & Exemplo \\
\hline pop & morrer & $\begin{array}{l}\text { João i-pop-Ø. } \\
\text { João PART-morrer-ABS } \\
\text { 'O João morreu' }\end{array}$ \\
\hline otam & chegar & $\begin{array}{l}\text { João i-otam- } \varnothing . \\
\text { João PART-chegar-ABS } \\
\text { 'O João chegou' }\end{array}$ \\
\hline $\begin{array}{l}\text { typ Antônio } \\
\text { Bigode }\end{array}$ & $\begin{array}{l}\text { encontrar } \\
\text { Antônio } \\
\text { Bigode }\end{array}$ & $\begin{array}{l}\text { João Ø-naka-typ-Ø } \quad \text { Antônio Bigode. } \\
\text { João 3-DECL-encontrar-NFUT Antônio Bigode } \\
\text { 'O João encontrou o Antônio Bigode' }\end{array}$ \\
\hline $\begin{array}{l}\text { otam myhint } \\
\text { deso ohyn } \\
\text { sok }\end{array}$ & $\begin{array}{l}\text { alcançar o } \\
\text { topo de uma } \\
\text { montanha }\end{array}$ & $\begin{array}{l}\text { João i-otam-Ø } \text { myhin-t deso } \\
\text { João PART-chegar-ABS } \text { um-OBL montanha topo } \text { em } \\
\text { 'O João chegou no topo de uma montanha' }\end{array}$ \\
\hline
\end{tabular}

48 Sintagmas verbais do tipo ir $+P P$ não são accomplishments canônicos. No entanto, eles podem ser considerados predicados que denotam eventos que têm uma duração e um telos definido. 


\subsubsection{Testes de Classes Acionais em Karitiana}

O objetivo desta subseção é aplicar nos predicados verbais do Karitiana apresentados acima os testes tradicionais que são encontrados na literatura para diagnóstico das classes acionais. O objetivo principal desses testes foi o de tentar encontrar um teste que fosse confiável para diagnosticar as classes acionais na língua. A divisão dos predicados verbais segundo sua classe acional será útil na análise da modificação de grau apresentada no próximo capítulo. Os testes propostos na literatura dizem respeito normalmente à (in)compatibilidade com certos adjuntos, à (im)posssibilidade de se fazer certas inferencias, e à (in)compatibilidade com certos tempos verbais. A maior parte dos testes associados ao tempo verbal foram propostos por Vendler (1957), uma vez que como vimos, o autor fez uma tipologia dos predicados verbais de acordo com sua relação com o tempo. Os outros testes utilizados foram discutidos nos trabalhos clássicos de Dowty (1979), Parsons (1990) e Chierchia (2003).

Como ficará claro ao longo da seção, a maior parte dos testes propostos pela literatura não se aplica totalmente em Karitiana. Parte deles não é adequada porque a língua possui certas características particulares que a diferencia muito do inglês. A expressão do tempo e do aspecto, por exemplo, interfere em muitos testes. Como se verá, o fato de em Karitiana o morfema de não futuro poder ser utilizado tanto para expressar um evento perfectivo quanto um evento imperfectivo interfere diretamente na inaplicabilidade de certos testes.

No entanto, mesmo quando os testes não se aplicam completamente, há indícios de que a distinção télico-atélico existe na língua. Como se verá, os contextos e traduções apontam que essa distinção é relevante. Além disso, há pelo menos um teste que pode ser aplicado com segurança para diferenciar os predicados télicos dos atélicos: o da ambiguidade com quase.

O teste mais clássico encontrado na literatura para a distinção entre predicados télicos e atélicos é a distribuição dos adjuntos em X tempo e por X tempo. Conforme mostram os exemplos em (139), abaixo, em $X$ tempo só pode ser usado com predicados télicos, enquanto que por $X$ tempo só pode ser usado com predicados atélicos. 
(139) a. O João correu por duas horas.

b. $\quad *$ O João correu em duas horas.

c. *O João desenhou um círculo por duas horas.

d. O João desenhou um círculo em duas horas.

Tabela 15: Teste em/por X tempo

\begin{tabular}{|l|c|c|}
\hline \multicolumn{1}{|c|}{ Tipo de Predicado } & em X Tempo & por X tempo \\
\hline télico & $\checkmark$ & $x$ \\
\hline atélico & $x$ & $\checkmark$ \\
\hline
\end{tabular}

Esse teste é tradicionalmente utilisado para distinguir predicados de accomplishment e atividades. O teste foi aplicado em Karitiana nesses predicados e também nos estativos porque o teste fornece pistas interessantes para a investigação desse tipo de predicado, como será discutido mais abaixo. O teste não se aplica a predicados de achievement porque são instantâneos.

A aplicação do teste em Karitiana mostrou que a distinção em-por $X$ tempo não se aplica na língua porque ela não diferencia os dois tipos de adjunto. Não há, em Kariana, duas posposições correspondentes a em e por. Os sintagmas que representam duração do evento são adjungidos sem posposição e podem representar as duas noções. Além disso, o morfema de não futuro na língua pode ser usado para expressar um evento perfectivo ou imperfectivo. Assim, a sentença João ipykynat pode ser traduzida tanto por 'O João correu', quanto 'O João ficou correndo', sendo compatível, portanto, tanto com as noções de 'em duas horas' quanto 'por duas horas'; ambas representados por sypomp horat. Esse teste, portanto, não é útil em Karitiana. A tabela abaixo mostra a tentativa. 
Tabela 16: Teste em/por X tempo em Karitiana (Atividades e Accomplishments)

\begin{tabular}{|c|c|c|}
\hline Verbo & Adjunto & Exemplo \\
\hline \multicolumn{3}{|r|}{ Atividades } \\
\hline \multirow[t]{2}{*}{$\begin{array}{l}\text { pykyn } \\
\text { 'correr' }\end{array}$} & em duas horas & $\begin{array}{l}\text { João i-pykyn-<a>-t sypom-p hora-t. } \\
\text { João PART-correr-ABS dois-OBL hora-ADV } \\
\text { 'O João ficou correndo em duas horas' }\end{array}$ \\
\hline & por duas horas & 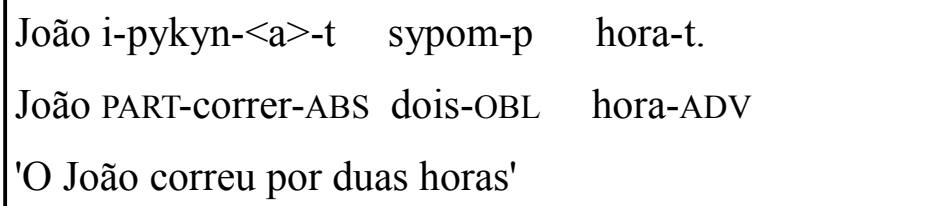 \\
\hline \multirow[t]{2}{*}{$\begin{array}{l}\text { pytim'adn } \\
\text { 'trabalhar' }\end{array}$} & em duas horas & $\begin{array}{l}\text { João i-pytim'adn-<a>-t sypom-p hora-t. } \\
\text { João PART-trabalhar-ABS dois-OBL hora-ADV } \\
\text { 'O João ficou trabalhando em duas horas' }\end{array}$ \\
\hline & por duas horas & $\begin{array}{l}\text { João i-pytim'adn-<a>-t sypom-p hora-t. } \\
\text { João PART-trabalhar-ABS dois-OBL hora-ADV } \\
\text { 'O João trabalhou por duas horas' }\end{array}$ \\
\hline \multirow[t]{2}{*}{$\begin{array}{l}\text { pimbik gooj } \\
\text { 'empurrar } \\
\text { carro' }\end{array}$} & em duas horas & $\begin{array}{l}\text { João Ø-na-pimbik-Ø gooj sypom-p hora-t. } \\
\text { João 3-DECL-empurrar-NFUT carro dois-OBL hora-ADV } \\
\text { 'O João ficou empurrando carro em duas horas' }\end{array}$ \\
\hline & por duas horas & $\begin{array}{l}\text { João Ø-na-pimbik-Ø } \quad \text { gooj sypom-p hora-t. } \\
\text { João 3-DECL-empurrar-NFUT carro dois-OBL hora-ADV } \\
\text { 'O João empurrou carro por duas horas' }\end{array}$ \\
\hline \multirow[t]{2}{*}{$\begin{array}{l}\text { tagngã gooj } \\
\text { 'dirigir carro' }\end{array}$} & em duas horas & $\begin{array}{l}\text { João Ø-na-tagngã-t gooj sypom-p hora-t. } \\
\text { João 3-DECL-dirigir-NFUT carro dois-OBL hora-ADV } \\
\text { 'O João ficou dirigindo carro em duas horas' }\end{array}$ \\
\hline & por duas horas & $\begin{array}{l}\text { João Ø-na-tagngã-t } \quad \text { gooj sypom-p } \\
\text { João 3-DECL-dirigir-NFUT carro dois-OBL } \\
\text { 'O João-ADV dirigiu carro por duas horas' }\end{array}$ \\
\hline
\end{tabular}




\begin{tabular}{|c|c|c|}
\hline & & Accomplishment \\
\hline kokot Sete de & em duas horas & João i-kokot- $\varnothing \quad$ Sete de Setembro dewota \\
\hline Setembro & & João PART-passar-ABS Sete de Setembro outro.lado \\
\hline dewota kyyn & & kyyn sypom-p hora-t. \\
\hline 'atravessar a & & para dois-OBL hora-ADV \\
\hline Sete de & & 'O João atravessou a rua Sete de Setembro em duas horas' \\
\hline Setembro' & & (ele terminou de atravessar) \\
\hline & por duas horas & João i-kokot- $\varnothing \quad$ Sete de Setembro dewota \\
\hline & & João PART-passar-ABS Sete de Setembro outro.lado \\
\hline & & kyyn sypom-p hora-t. \\
\hline & & para dois-OBL hora-ADV \\
\hline & & $\begin{array}{l}\text { 'O João ficou atravessando a rua Sete de Setembro por duas } \\
\text { horas' }\end{array}$ \\
\hline & & (demorou porque os carros ficaram atrapalhando) \\
\hline pog руејера & em duas horas & João $\varnothing$-naka-pog-<a>-t $\quad$ pyejepa \\
\hline goot & & João 3-DECL-pintar.de.branco-NFUT escola \\
\hline 'pintar a & & goot sypom-p hora-t. \\
\hline escola nova & & novo dois-OBL hora-ADV \\
\hline de branco' & & 'O João pintou a escola nova de branco em duas horas' \\
\hline & & (ele terminou de pintar) \\
\hline & por duas horas & João $\varnothing$-naka-pog-<a>-t pyejepa \\
\hline & & João 3-DECL-pintar.de.branco-NFUT escola \\
\hline & & goot sypom-p hora-t. \\
\hline & & novo dois-OBL hora-ADV \\
\hline & & 'João ficou pintando escola nova de branco por duas horas' \\
\hline tat Porto & em duas horas & João itat $\quad$ Porto Velho pip sypom-p hora-t. \\
\hline 'ir para Porto & & João PART-ir-ABS Porto Velho para dois-OBL hora-ADV \\
\hline Velho' & & 'O Joao to1 para Porto Velho em duas horas' \\
\hline & por duas horas & João itat $\quad$ Porto Velho pip sypom-p hora-t. \\
\hline & & João PART-ir-ABS Porto Velho para dois-OBL hora-ADV \\
\hline & & 'O João ficou indo para Porto Velho em duas horas' \\
\hline
\end{tabular}




\begin{tabular}{|c|c|c|}
\hline \multirow[t]{2}{*}{$\begin{array}{l}\text { 'a myhint boet } \\
\text { 'fazer um } \\
\text { colar' }\end{array}$} & em duas horas & $\begin{array}{l}\text { João Ø-naka-m-'a-t myhin-t boet } \\
\text { João 3-DECL-CAUS-fazer-NFUT um-OBL colar } \\
\text { sypom-p hora-t. } \\
\text { dois-OBL hora-ADV } \\
\text { 'O João fez um colar em duas horas' }\end{array}$ \\
\hline & por duas horas & $\begin{array}{l}\text { João Ø-naka-m-'a-t myhin-t boet } \\
\text { João 3-DECL-CAUS-fazer-NFUT um-OBL colar } \\
\text { sypom-p hora-t. } \\
\text { dois-OBL hora-ADV } \\
\text { 'O João ficou fazendo um colar por duas horas' }\end{array}$ \\
\hline
\end{tabular}

A tentativa do teste mostrou, no entanto, que há um indicativo de que as classes de verbos são diferentes. Conforme mostra a tabela abaixo, os predicados estativos stage-level quando são usados com sypomp horat 'duas horas' apresentam apenas tradução de 'por duas horas'. O que é esperado para essa classe é que ela seja compatível apenas por adjuntos do tipo por duas horas, então o resultado está dentro das expectativas. Os predicados estativos individual-level, por outro lado, são normamente incompatíveis com adjuntos de qualquer tipo, conforme discutido na seção 1.2.4. A tabela abaixo mostra que essa incompatibilidade é encontrada também em Karitiana. O verbo aka Karitiana 'ser Karitiana' não é compatível com sypomp gõgõrongant 'dois anos'. O verbo pypyyt Matematica-ty 'saber Matemática' é possível com o adjunto, mas há uma mudança no significado do verbo, que passa a ser interpretado como 'aprender Matemática'. O verbo kywytidn Botỹjty 'acreditar em Deus' é possível com o adjunto também se sofrer uma mudança de significado. $\mathrm{O}$ uso de sypomp gõgõrongant com tradução de 'em dois anos' é possível se o verbo tiver uma interpretação inceptiva ('começou a acreditar em Deus') e com a tradução de 'por dois anos' é possível se o verbo for interpretado como um predicado stage-level ${ }^{49}$.

49 O comportamento é semelhante em português:

(i) O João acreditou em Deus em dois anos. (interpretação inceptiva)

(ii) O João acreditou em Deus por dois anos. (interpretação stage-level) 
Tabela 17: Teste em/por X tempo em Karitiana (Estativos)

\begin{tabular}{|c|c|c|}
\hline \multicolumn{3}{|r|}{ Estativos Stage-level } \\
\hline \multirow{2}{*}{$\begin{array}{l}\text { siki'y } \\
\text { 'querer' }\end{array}$} & em duas horas & Tradução não disponível \\
\hline & por duas horas & $\begin{array}{l}\text { João i-siki'y-t ese-ty sypom-p hora-t. } \\
\text { João PART-querer-ABS água-OBL dois-OBL hora-ADV } \\
\text { 'O João ficou querendo água por duas horas' } \\
\text { (ficou duas horas sem beber) }\end{array}$ \\
\hline \multirow{2}{*}{$\begin{array}{l}\text { osedn } \\
\text { 'estar } \\
\text { alegre' }\end{array}$} & em duas horas & Tradução não disponível \\
\hline & por duas horas & $\begin{array}{l}\text { João i-osedn-Ø sypom-p hora-t. } \\
\text { João PART-estar.alegre-ABS dois-OBL hora-ADV } \\
\text { 'O João ficou feliz por duas horas' }\end{array}$ \\
\hline \multirow{2}{*}{$\begin{array}{l}\text { em } \\
\text { 'estar sujo' }\end{array}$} & em duas horas & Tradução não disponível \\
\hline & por duas horas & $\begin{array}{l}\text { João i-em-Ø sypom-p hora-t. } \\
\text { João PART-estar.sujo-ABS dois-OBL hora-ADV } \\
\text { 'O João ficou sujo por duas horas' }\end{array}$ \\
\hline \multirow{2}{*}{$\begin{array}{l}\text { pasadn } \\
\text { 'gostar' }\end{array}$} & em dois anos & Tradução não disponível \\
\hline & por dois anos & $\begin{array}{l}\text { João i-pasadn- } \varnothing \quad \text { Milena-ty } \quad \text { sypom-p } \\
\text { João PART-gostar-ABS Milena-OBL dois-OBL } \\
\text { gõgõrongan-t. } \\
\text { ano-ADV } \\
\text { 'O João gostou da Milena por dois anos' }\end{array}$ \\
\hline \multicolumn{3}{|r|}{ Estativos Individual-level } \\
\hline $\begin{array}{l}\text { aka } \\
\text { Karitiana } \\
\text { 'ser } \\
\text { Karitiana' }\end{array}$ & em dois anos & 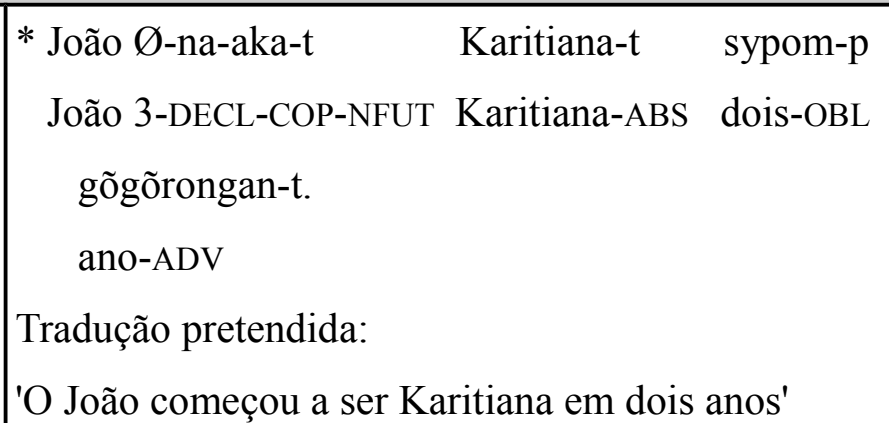 \\
\hline
\end{tabular}




\begin{tabular}{|c|c|c|}
\hline & por dois anos & $\begin{array}{l}\text { * João Ø-na-aka-t } \quad \text { Karitiana-t } \quad \text { sypom-p } \\
\text { João 3-DECL-COP-NFUT Karitiana-ABS } \\
\text { gõgõrongan-t. } \\
\text { ano-ADV } \\
\text { Tradução pretendida: } \\
\text { 'O João foi Karitiana por dois anos' }\end{array}$ \\
\hline \multirow[t]{2}{*}{$\begin{array}{l}\text { pypyyt } \\
\text { Matematica } \\
\text { ty } \\
\text { 'saber } \\
\text { Matemática' }\end{array}$} & em dois anos & $\begin{array}{l}\text { João i-pypyyt- } \varnothing \quad \text { Matematica-ty } \\
\text { João PART-saber-ABS Matemática-OBL } \\
\text { sypom-p gõgõrongan-t. } \\
\text { dois-OBL ano-OBL } \\
\text { 'O João aprendeu Matemática em dois anos' } \\
\rightarrow \text { mudança no significado do verbo }\end{array}$ \\
\hline & por dois anos & $\begin{array}{l}\text { João i-pypyyt- } \varnothing \quad \text { Matematica-ty } \\
\text { João PART-saber-ABS Matemática-OBL } \\
\text { sypom-p gõgõrongan-t. } \\
\text { dois-OBL ano-OBL } \\
\text { 'O João ficou aprendendo Matemática por dois anos' } \\
\rightarrow \text { mudança no significado do verbo }\end{array}$ \\
\hline \multirow[t]{2}{*}{$\begin{array}{l}\text { kywytidn } \\
\text { Botỹjty } \\
\text { 'acreditar } \\
\text { em Deus' }\end{array}$} & em dois anos & $\begin{array}{l}\text { João i-kywytidn- }<\text { a }>\text {-t Botỹj-ty } \\
\text { João PART-acreditar-ABS Deus-OBL } \\
\text { sypom-p gogõrongan-t. } \\
\text { dois-OBL ano-OBL } \\
\text { 'Luciana acreditou em Deus em dois anos' } \\
\text { (depois de dois anos, passou a acreditar) } \\
\rightarrow \text { A sentença passa a ter uma interpretação inceptiva }\end{array}$ \\
\hline & por dois anos & $\begin{array}{l}\text { João i-kywytidn- }<\text { a }>-t \text { Botỹj-ty } \\
\text { João PART-acreditar-ABS Deus-OBL } \\
\text { sypom-p gõgõrongan-t. } \\
\text { dois-OBL ano-OBL } \\
\text { 'Luciana acreditou em Deus por dois anos' } \\
\text { (depois parou de acreditar) }\end{array}$ \\
\hline
\end{tabular}


Um outro teste muito utilizado para a separação de predicados télicos e atélicos diz respeito a inferências possíveis a partir da interrupção de uma ação. O conjunto de sentenças abaixo ilustra esse teste. Se um evento descrito por um predicado atélico como o descrito em (140a) for interrompido, há um acarretamento de (140a) a (140b). Mas, se um evento télico como o de (141a) for interrompido, não se pode afirmar que (141b) seja verdadeira. Ou seja, o acarretamento não se mantém. Essa caracterização ficou conhecida na literatura como paradoxo do imperfectivo.

a. O João estava correndo.

b. O João correu.

(141) a. O João estava estava desenhando um círculo.

b. O João desenhou um círculo.

Tabela 18: Teste do Paradoxo do Imperfectivo

\begin{tabular}{|l|c|}
\hline Tipo de Predicado & $\begin{array}{c}\text { João estava X-ndo } \\
\text { acarreta João X-eu }\end{array}$ \\
\hline télico & $x$ \\
\hline atélico & $\checkmark$ \\
\hline
\end{tabular}

Conforme dito anteriormente, em Karitiana, o tempo não futuro pode ter uma interpretação perfectiva ou imperfectiva. O capítulo 1 mostrou que a língua possui certas estratégias para expressar o imperfectivo. No entanto, parece não haver sido detectado na língua um meio de se expressar perfectividade ${ }^{50}$. Sem uma estratégia como essa, não é possível formular a segunda parte do acarretamento como correu e desenhou um círculo porque, em Karitiana, essas sentenças também podem ser usadas em situações do tipo 'ficou correndo' e 'ficou desenhando um círculo'.

A tabela 19, abaixo, mostra a tentativa de aplicação do teste a verbos de atividade e a accomplishments.

50 Storto (1999) glosa tykiri como aspecto perfectivo dentro das sentenças subordinadas (sentença (2) do capítulo 1 desta tese). No entanto, a autora não desenvolve uma análise para a marca. 
Tabela 19: Teste do Paradoxo do Imperfectivo em Karitiana

\begin{tabular}{|c|c|}
\hline Verbo & Exemplo \\
\hline \multicolumn{2}{|r|}{ Atividades } \\
\hline $\begin{array}{l}\text { pykyn } \\
\text { 'correr' }\end{array}$ & $\begin{array}{l}\text { João i-pykyn-<a>-t ty-ka-t. } \\
\text { João PART-correr-ABS IMP-MOV.SG-NFUT então } \\
\text { Ø-pyr-a-nanda-gn } \\
\text { 3-ASS-PASS-atropelar-NFUT ele } \\
\text { 'O João estava correndo. Então, ele foi atropelado' } \\
\text { João i-pykyn-<a>-t. } \\
\text { João PART-correr-ABS } \\
\text { 'O João correu' / 'O João ficou correndo' }\end{array}$ \\
\hline $\begin{array}{l}\text { pytim'adna } \\
\text { 'trabalhar' }\end{array}$ & $\begin{array}{l}\text { João i-pytim'adn-<a>-t ty-ka-t. } \\
\text { João PART-trabalhar-ABS IMP-MOV.SG-NFUT } \\
\text { Ø-naka-'ot- } \varnothing \quad \text { i. } \\
\text { 3-DECL-cair-NFUT ele }{ }^{51} \\
\text { 'O João estava trabalhando. Enquanto isso, ele caiu' } \\
\text { João i-pytim'adn-<a>-t. } \\
\text { João PART-trabalhar-ABS } \\
\text { 'O João trabalhou' / 'O João ficou trabalhando' }\end{array}$ \\
\hline $\begin{array}{l}\text { pimbik gooj } \\
\text { 'empurrar carro' }\end{array}$ & 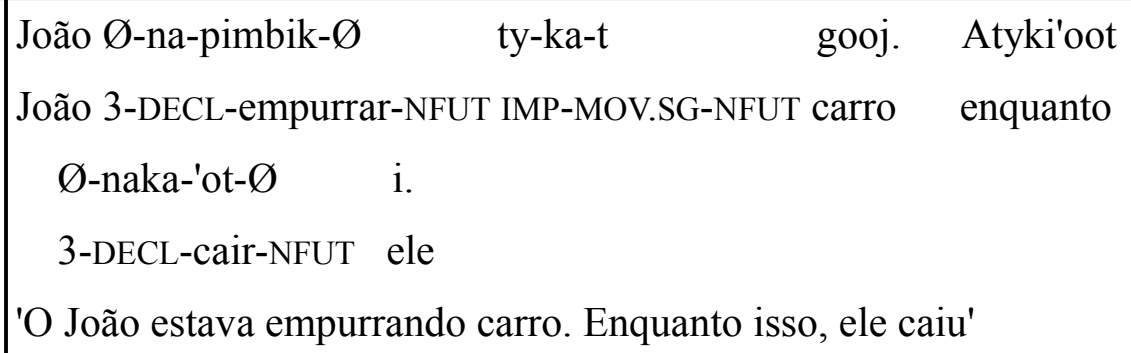 \\
\hline
\end{tabular}

51 A princípio, as conjunções sentenciais atykiri e atyki'oot parecem ser intercambiáveis nesses contextos. As palavras parecem ser polimorfêmicas. Elas poderiam ser glosadas como a-ty-ki-ri e a-ty-ki-'oot. Uma vez que não possuímos ainda uma análise para esses morfemas, preferimos deirar as palavras sem glosas em nossos exemplos. Storto $(2012 \mathrm{~b}, 2013)$ apresenta uma análise para os aspectuais tykiri e tyki'oot de onde essas conjunções podem ter sido derivadas. Ver Storto (2012b, 2013) para mais detalhes. 


\begin{tabular}{|c|c|}
\hline & $\begin{array}{l}\text { João Ø-na-pimbik- } \varnothing \quad \text { gooj. } \\
\text { João 3-DECL-empurrar-NFUT carro } \\
\text { 'O João empurrou carro' / 'O João ficou empurrando carro' }\end{array}$ \\
\hline $\begin{array}{l}\text { tagngã gooj } \\
\text { 'dirigir carro' }\end{array}$ & $\begin{array}{l}\text { João Ø-naka-tagngã-t } \quad \text { ty-ka-t } \quad \text { gooj. } \\
\text { João 3-DECL-dirigir-NFUT } \\
\text { AMP-MOV.SG-NFUT carro } \\
\text { então } \quad \text { 3-DECL-acabar-Ø } \\
\text { 'O João estava dirigindo carro. Então, acabou a gasolina' } \\
\text { João Ø-naka-tagngã-t } \quad \text { gooj. } \\
\text { João 3-DECL-dirigir-NFUT carro } \\
\text { 'O João dirigiu carro' / 'O João ficou dirigindo carro' }\end{array}$ \\
\hline & Accomplishment \\
\hline $\begin{array}{l}\text { kokot Sete de } \\
\text { Setembro } \\
\text { dewota kyyn } \\
\text { 'atravessar a } \\
\text { Sete de } \\
\text { Setembro' }\end{array}$ & 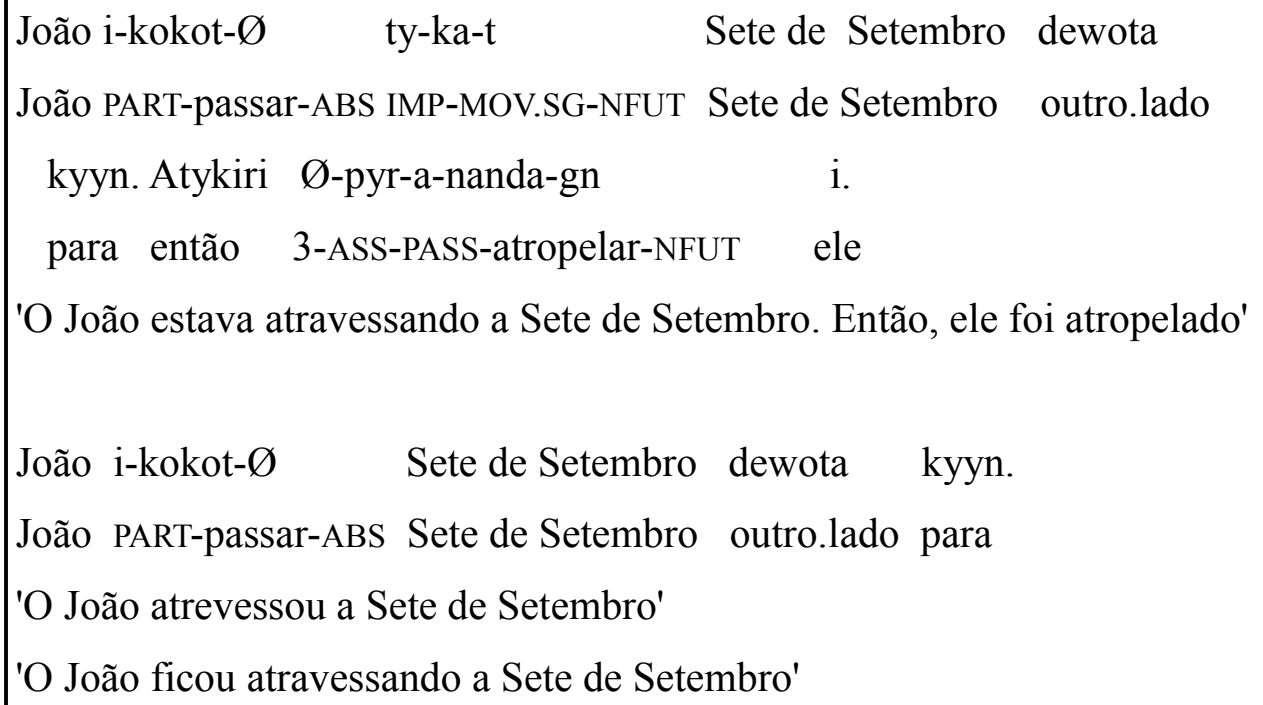 \\
\hline $\begin{array}{l}\text { pog pyejepa } \\
\text { goot } \\
\text { 'pintar a escola } \\
\text { nova de branco' }\end{array}$ & 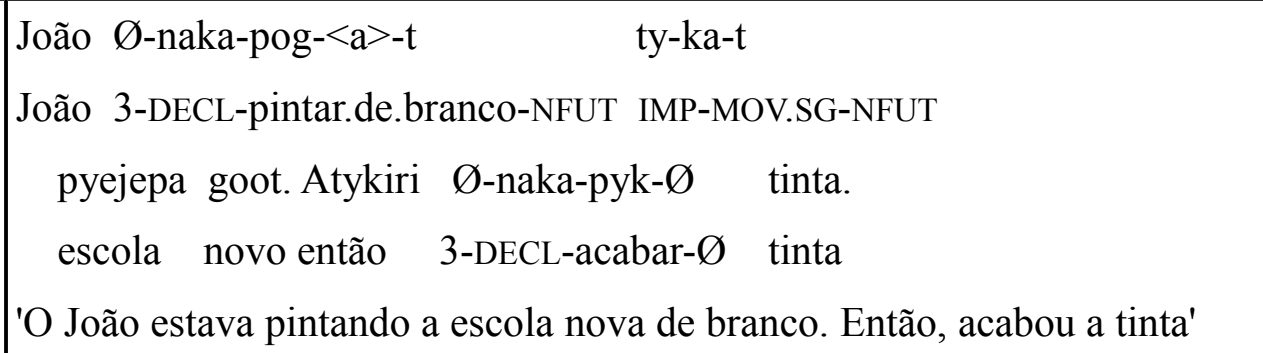 \\
\hline
\end{tabular}




\begin{tabular}{|c|c|}
\hline & $\begin{array}{l}\text { João } \varnothing \text {-naka-pog-<a>-t pyejepa goot. } \\
\text { João 3-DECL-pintar.de.branco-NFUT escola novo } \\
\text { 'O João pintou a escola nova de branco' } \\
\text { 'O João ficou pintando a escola nova de branco' }\end{array}$ \\
\hline $\begin{array}{l}\text { tat Porto Velho } \\
\text { pip } \\
\text { 'ir para Porto } \\
\text { Velho' }\end{array}$ & 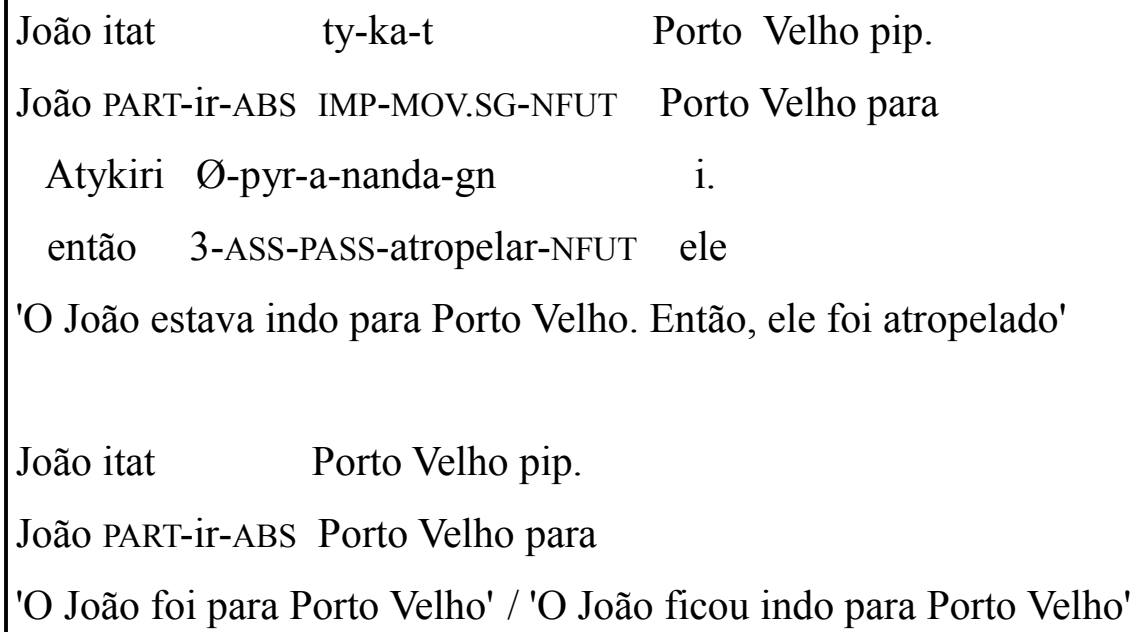 \\
\hline $\begin{array}{l}\text { 'a myhint boet } \\
\text { 'fazer um colar' }\end{array}$ & 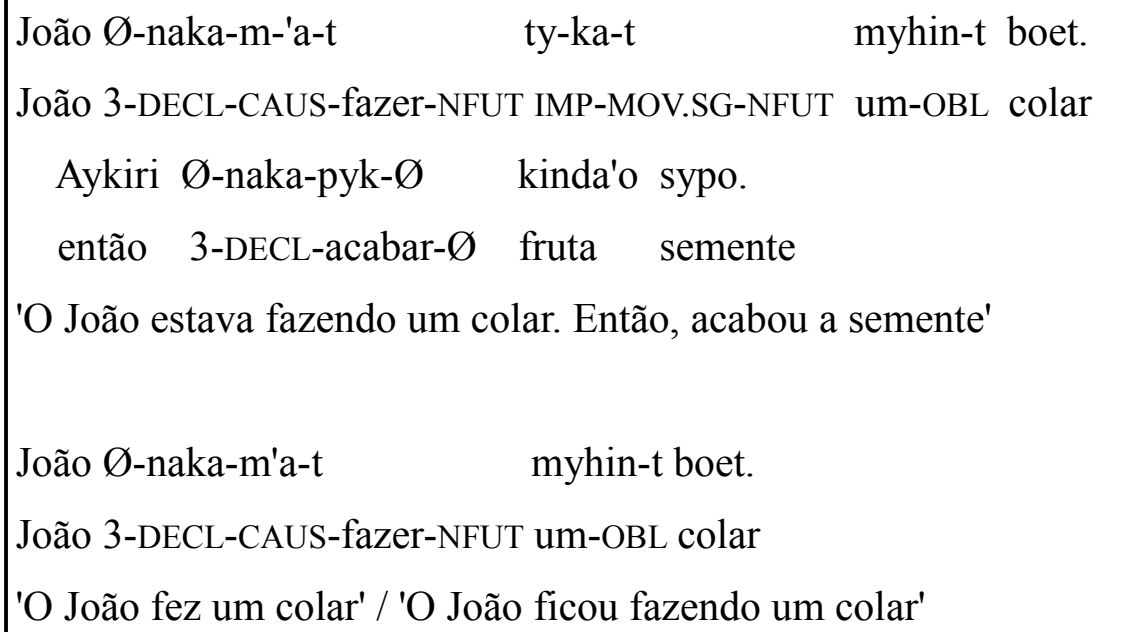 \\
\hline
\end{tabular}

Duas estratégias foram utilizadas para tentar salvar o teste do paradoxo imperfectivo em Karitiana. A primeira delas foi checar o contexto de uso das sentenças. A tabela abaixo mostra que os consultores confirmam que as sentenças com predicados de accomplishment e interrupção da ação descrevem um evento que não foi concretizado. 
Tabela 20: Interrupção de Predicados de Accomplishment em Karitiana

\begin{tabular}{|c|c|}
\hline Verbo & Exemplo \\
\hline kokot Sete de & Sete de Setembro dewota \\
\hline Setembro & João PART-passar-ABS IMP-MOV.SG-NFUT Sete de Setembro outro.lado \\
\hline dewota kyyn & kyyn. Atykiri $\varnothing$-pyr-a-nanda-gn \\
\hline 'atravessar a & para então 3-ASS-PASS-atropelar-NFUT \\
\hline Sete de & 'O João estava atravessando a Sete de Setembro. Então, ele foi atropelado' \\
\hline Setembro' & $\checkmark$ não conseguiu atravessar \\
\hline pog руејера & João Ø-naka-pog-<a $>$-t $\quad$ ty-ka-t \\
\hline & João 3-DECL-pintar.de.branco-NFUT IMP-MOV.SG-NFUT \\
\hline 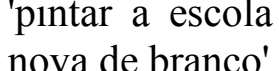 & pyejepa goot. Atykiri $\varnothing$-naka-pyk- $\varnothing$ tinta. \\
\hline & escola novo então 3-DECL-acabar- $\varnothing$ tinta \\
\hline & 'O João estava pintando a escola nova de branco. Então, acabou a tinta' \\
\hline & Contexto: $\quad \checkmark$ não conseguiu pintar toda a escola \\
\hline tat Porto Velho & João itat $\quad$ ty-ka-t $\quad$ Porto Velho pip. \\
\hline & João PART-ir-ABS IMP-MOV.SG-NFUT Porto Velho para \\
\hline Velho' & Atykiri $\varnothing$-pyr-a-nanda-gn $\quad$ i. \\
\hline & então 3-ASS-PASS-atropelar-NFUT ele \\
\hline & 'O João estava indo para Porto Velho. Então, ele foi atropelado' \\
\hline & Contexto: $\quad \checkmark \quad$ não conseguiu chegar \\
\hline 'at myhint boet & João Ø-naka-m'a-t $\quad$ ty-ka-t \\
\hline 'fazer um colar' & João 3-DECL-CAUS-fazer-NFUT IMP-MOV.SG-NFUT um-OBL colar \\
\hline & Atykiri $\varnothing$-naka-pyk- $\quad$ kinda'o sypo. \\
\hline & então 3-DECL-acabar- $\varnothing$ fruta semente \\
\hline & 'O João estava fazendo um colar. Então, acabou a semente' \\
\hline & Contexto: $\quad \checkmark \quad$ não conseguiu terminar \\
\hline
\end{tabular}

A tabela abaixo, por sua vez, mostra que os verbos de atividade não apresentam o mesmo comportamento. Eventos descritos por verbos de atividade podem ser interrompidos sem prejuízo para a concretização do evento. 
Tabela 21: Interrupção de Predicados de Atividade em Karitiana

\begin{tabular}{|c|c|}
\hline Verbo & Exemplo \\
\hline $\begin{array}{l}\text { pykyn } \\
\text { 'correr' }\end{array}$ & $\begin{array}{ll}\text { João i-pykyn- }<\text { a }>\text {-t } \quad \text { ty-ka-t. } & \text { Atykiri } \\
\text { João PART-correr-ABS IMP-MOV.SG-NFUT } & \text { então } \\
\text { Ø-pyr-a-nanda-gn } & \text { i. } \\
\text { 3-ASS-PASS-atropelar-NFUT ele } & \\
\text { 'O João estava correndo. Então, ele foi atropelado' } \\
\text { Contexto: } \quad \checkmark \text { correu }\end{array}$ \\
\hline $\begin{array}{l}\text { pytim'adn } \\
\text { 'trabalhar' }\end{array}$ & $\begin{array}{l}\text { João i-pytim'adn- }<\text { a }>\text {-t } \text { ty-ka-t. } \\
\text { João PART-trabalhar-ABS IMP-MOV.SG-NFUT } \\
\begin{array}{l}\text { Ø-naka-'ot- } \varnothing \quad \text { i. } \\
\text { 3-DECL-cair-NFUT }\end{array} \\
\text { 'O João estava trabalhando } \\
\text { Contexto: } \quad \checkmark \quad \text { trabalhou }\end{array}$ \\
\hline $\begin{array}{l}\text { pimbik gooj } \\
\text { 'empurrar carro' }\end{array}$ & $\begin{array}{l}\text { João } Ø \text {-na-pimbik- } \varnothing \quad \text { ty-ka-t } \quad \text { gooj. Atyki'oot } \\
\text { João 3-DECL-empurrar-NFUT IMP-MOV.SG-NFUT carro enquanto } \\
\text { Ø-naka-'ot- } \quad \text { i. } \\
\text { 3-DECL-cair-NFUT ele } \\
\text { 'O João estava empurrando carro. Enquanto isso, ele caiu' } \\
\text { Contexto: } \quad \checkmark \text { empurrou carro }\end{array}$ \\
\hline $\begin{array}{l}\text { tagngã gooj } \\
\text { 'dirigir carro' }\end{array}$ & $\begin{array}{l}\text { João } \varnothing \text {-naka-tagngã-t } \quad \text { ty-ka-t } \quad \text { gooj. } \\
\text { João 3-DECL-dirigir-NFUT } \\
\text { AMP-MOV.SG-NFUT carro } \\
\text { Atykiri } \quad \text { Ø-naka-pyk-Ø } \quad \text { gasolina. } \\
\text { então } \quad \text { 3-DECL-acabar-Ø gasolina } \\
\text { 'O João estava dirigindo carro. Então, acabou a gasolina' } \\
\text { Contexto: } \quad \checkmark \quad \text { dirigiu carro }\end{array}$ \\
\hline
\end{tabular}

Uma outra estratégia utilizada para tentar aplicar o teste do paradoxo do imperfectivo em Karitiana foi formular uma sentença que significasse algo tipo 'mas não terminou de'. A tabela abaixo mostra a aplicação dessa estratégia. 
Tabela 22: Interrupção de Predicados de Accomplishment em Karitiana com Descrição Complementar

\begin{tabular}{|c|c|}
\hline Verbo & Exemplo \\
\hline $\begin{array}{l}\text { kokot Sete de } \\
\text { Setembro } \\
\text { dewota kyyn } \\
\text { 'atravessar a } \\
\text { Sete de } \\
\text { Setembro' }\end{array}$ & 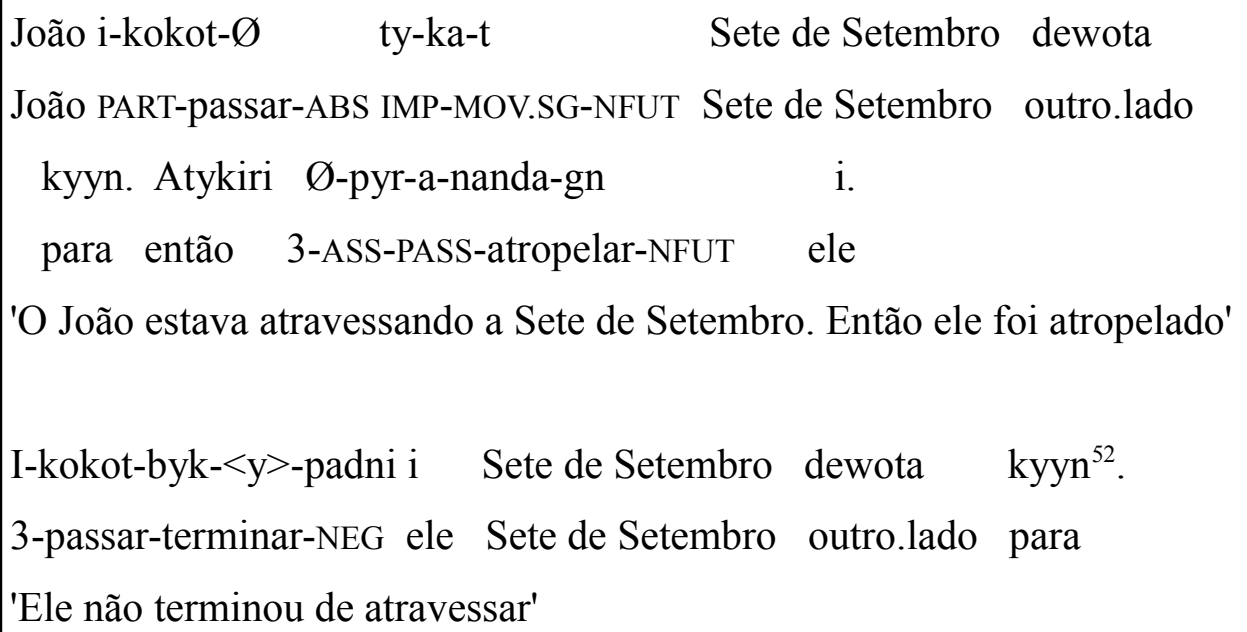 \\
\hline $\begin{array}{l}\text { pog pyejepa } \\
\text { goot } \\
\text { 'pintar a escola } \\
\text { nova de branco' }\end{array}$ & $\begin{array}{l}\text { João } \varnothing \text {-naka-pog-<a>-t ty-ka-t } \\
\text { João 3-DECL-pintar.de.branco-NFUT IMP-MOV.SG-NFUT } \\
\text { pyejepa goot. Atykiri } \varnothing \text {-naka-pyk- } \varnothing \text { tinta. } \\
\text { escola novo então } \quad \text { 3-DECL-acabar-Ø tinta } \\
\text { 'O João estava pintando a escola nova de branco. Então, acabou a tinta' } \\
\text { I-pog-byk-<y>-padni pyjepa goot i. } \\
\text { 3-pintar.de.branco-terminar-NEG escola novo ele } \\
\text { 'Ele não terminou de pintar a escola nova de branco' }\end{array}$ \\
\hline $\begin{array}{l}\text { tat Porto Velho } \\
\text { pip } \\
\text { 'ir para Porto } \\
\text { Velho' }\end{array}$ & 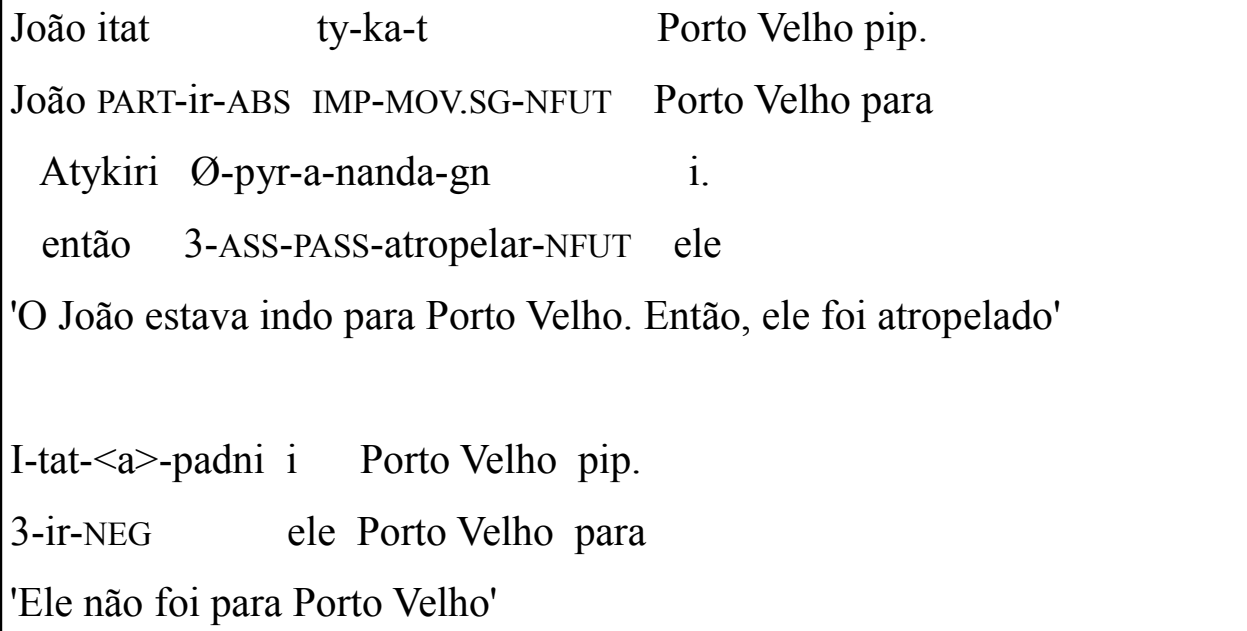 \\
\hline
\end{tabular}

${ }_{52} \mathrm{O}$ infixo $\{-b y k-\}$ é semelhante a um aspectual com significado de 'terminar de'. 


\begin{tabular}{|c|c|}
\hline & $\begin{array}{l}\text { *I-tat-byk-<y>-padni i Porto Velho pip. } \\
\text { 3-ir-terminar-NEG ele Porto Velho para } \\
\text { Tradução pretendida: 'Ele não terminou de chegar em Porto Velho' } \\
\rightarrow \text { Assim como em português, em Karitiana, não se pode dizer algo do tipo } \\
\text { 'terminou de } \text { ir }^{153} \text {. }\end{array}$ \\
\hline $\begin{array}{l}\text { 'at myhint boet } \\
\text { 'fazer um colar' }\end{array}$ & $\begin{array}{l}\text { João } \varnothing \text {-naka-m-'a-t } \quad \text { ty-ka-t } \\
\text { João 3-DECL-CAUS-fazer-NFUT IMP-MOV.SG-NFUT um-OBL colar } \\
\text { Atykiri } \varnothing \text {-naka-pyk- } \varnothing \quad \text { kinda'o sypo. } \\
\text { então } \quad 3 \text {-DECL-acabar- } \varnothing \text { fruta semente } \\
\text { 'O João estava fazendo um colar. Então, acabou a semente' } \\
\text { I-m-'a-byk-<y>-padni i myhin-t boet. } \\
\text { 3-CAUS-fazer-terminar-NEG ele um-OBL colar } \\
\text { 'Ele não terminou de fazer um colar' }\end{array}$ \\
\hline
\end{tabular}

A tabela abaixo mostra a mesma estratégia mas sem interrupção explícita da ação e com uma sequência do tipo 'então ainda não atravessou'.

$\overline{53}$ Isso se deve ao fato de que $i r+P P$ não é um verbo de accomplishment canônico. 
Tabela 23: Interrupção de Predicados de Accomplishment em Karitiana com

Descrição Complementar com sapadni 'ainda não'

\begin{tabular}{|c|c|}
\hline Verbo & Exemplo \\
\hline $\begin{array}{l}\text { kokot Sete de } \\
\text { Setembro } \\
\text { dewota kyyn }\end{array}$ & 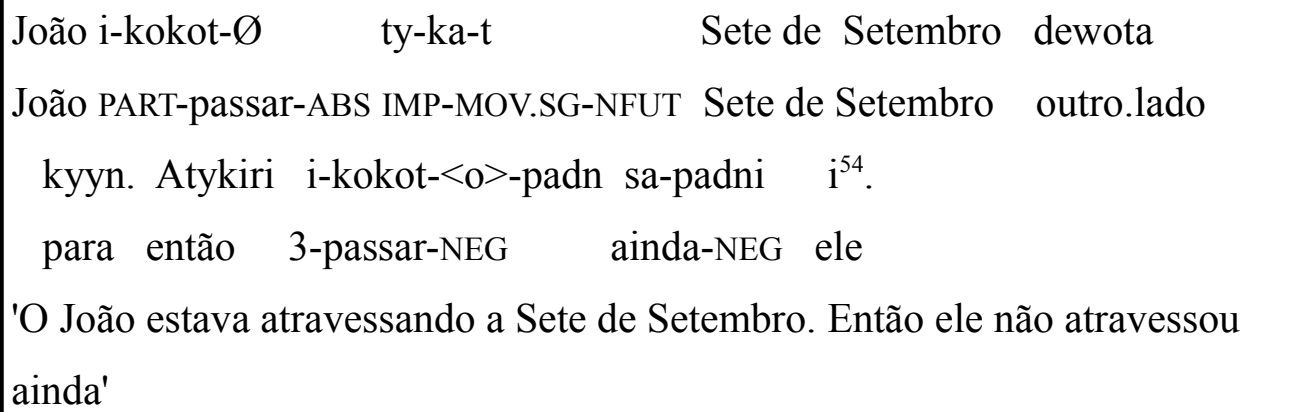 \\
\hline $\begin{array}{l}\text { pog pyejepa } \\
\text { goot } \\
\text { 'pintar a escola } \\
\text { nova de } \\
\text { branco' }\end{array}$ & 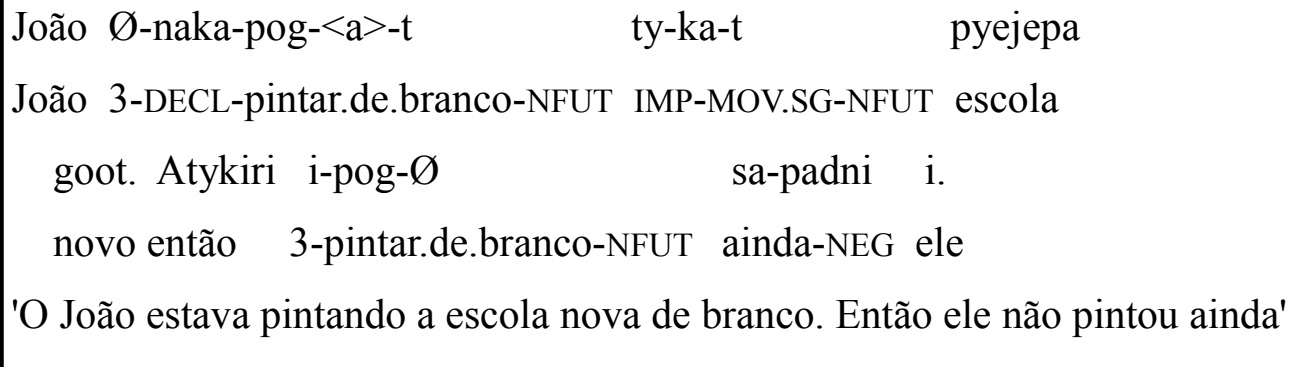 \\
\hline $\begin{array}{l}\text { tat Porto Velho } \\
\text { pip } \\
\text { 'ir para Porto } \\
\text { Velho' }\end{array}$ & 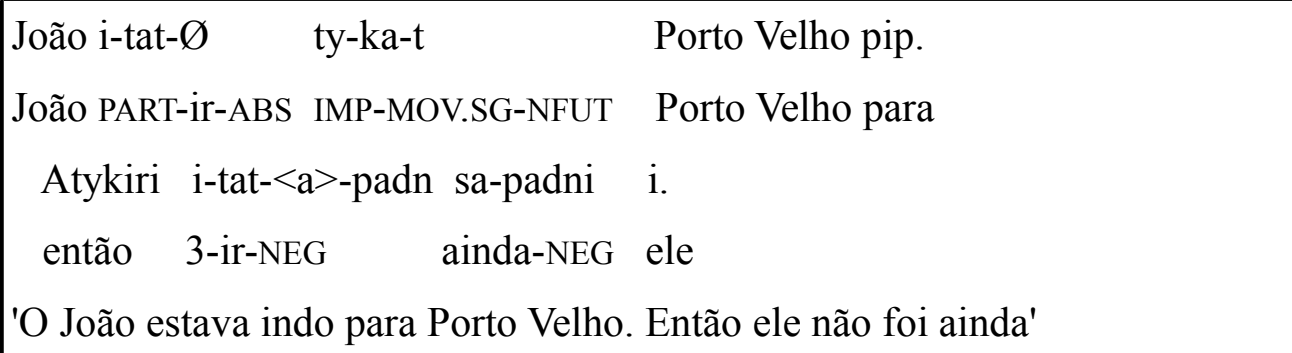 \\
\hline $\begin{array}{l}\text { 'at myhint boet } \\
\text { 'fazer um colar' }\end{array}$ & 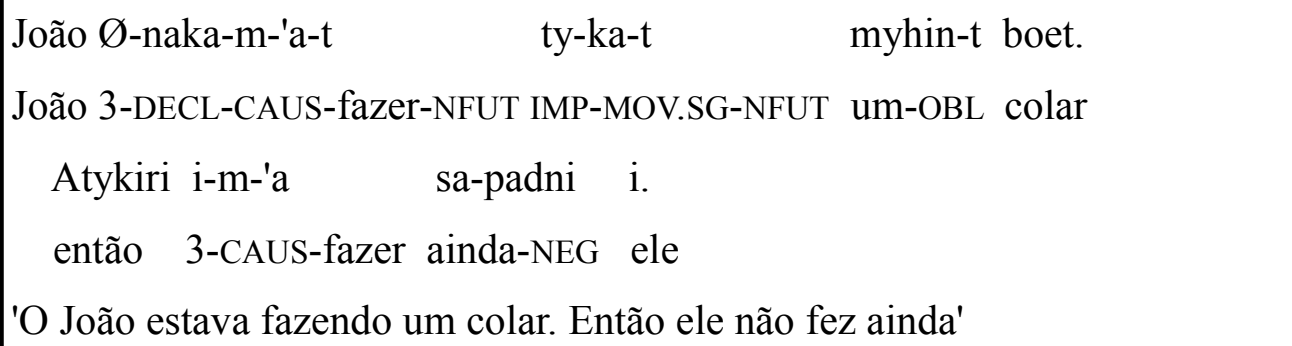 \\
\hline
\end{tabular}

As estratégias acima mostraram que há uma diferença entre predicados de atividade e de accomplishment em Karitiana, indicando que a distinção télico-atélico pode ser relevante na língua. $\mathrm{O}$ teste com 'então ainda não' foi aplicado também em predicados de achievement, com o mesmo resultado obtido com os accomplishments (ver tabela abaixo). Isso indica que esse é um bom teste para separar predicados télicos de atélicos na língua.

54 Estamos considerando que sapadni tem uma concordância negativa. 
Tabela 24: Interrupção de Predicados de Achievement em Karitiana com

Descrição Complementar com sapadni 'ainda não'

\begin{tabular}{|c|c|}
\hline Verbo & Exemplo \\
\hline $\begin{array}{l}\text { рор } \\
\text { 'morrer' }\end{array}$ & $\begin{array}{l}\text { João i-pop-Ø } \quad \text { ty-ka-t. } \quad \text { Aykiri } \\
\text { João PART-morrer-ABS IMP-MOV.SG-NFUT então } \\
\text { i-pop-<o>-padni sa-padni i. } \\
\text { 3-morrer-NEG ainda-NEG ele } \\
\text { 'O João está morrendo. Então, ele não morreu ainda' }\end{array}$ \\
\hline $\begin{array}{l}\text { otam } \\
\text { 'chegar' }\end{array}$ & $\begin{array}{l}\text { João i-otam- } \quad \text { ty-ka-t. } \quad \text { Atykiri } \\
\text { João PART-chegar-ABS IMP-MOV.SG-NFUT então } \\
\begin{array}{lll}\text { i-otam sa-padni } \quad \text { i. } \\
\text { 3-chegar ainda-NEG } & \text { ele } \\
\text { 'O João está chegando. Então ele não chegou ainda' }\end{array}\end{array}$ \\
\hline $\begin{array}{l}\text { typ Antônio } \\
\text { Bigode } \\
\text { 'encontrar } \\
\text { Antônio Bigode' }\end{array}$ & 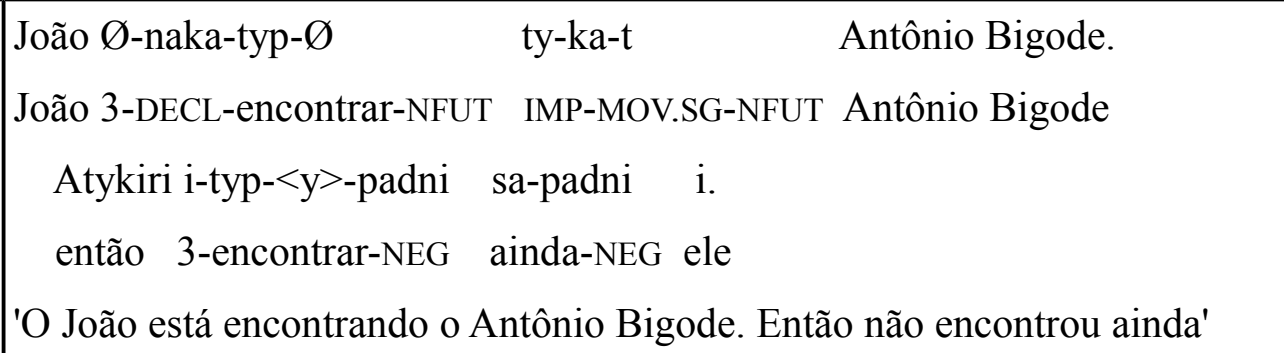 \\
\hline $\begin{array}{l}\text { otam myhint } \\
\text { deso ohyn sok } \\
\text { 'chegar no topo } \\
\text { de uma } \\
\text { montanha' }\end{array}$ & 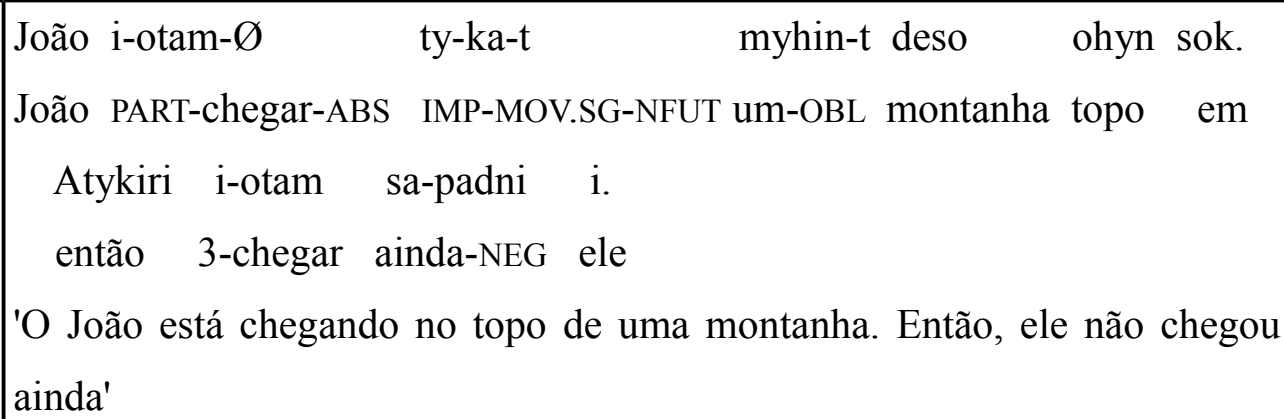 \\
\hline
\end{tabular}

As tabelas acima mostraram que, embora não seja possível formular o teste do paradoxo do imperfectivo exatamente como ele é utilizado em línguas como o inglês e o português, é possível reformular o teste de forma a mostrar que a divisão de predicados verbais em télicos e atélicos é pertinente na língua.

Conforme apresentado no início deste capítulo, um dos testes utilizados para diagnosticar verbos estativos e de acchievement em inglês é a incompatibilidade com o 
progressivo, um tempo contínuo (Vendler 1957). Os exemplos (107) e (108), repetidos abaixo em (142) ilustram essa imcompatibilidade.

a. I'm running.

ATIVIDADE

'Eu estou correndo'

b. I'm drawing a circle.

ACCOMPLISHMENT

'Eu estou desenhando um círculo'

c. $\quad$ * I'm recognizing it.

ACHIEVEMENT

'Eu estou reconhecendo'

d. * I'm loving it.

ESTADO

'Eu estou amando'

(exemplos de Vendler, 1957, p. 144)

Tabela 25: Teste da Compatibilidade com o Progressivo

\begin{tabular}{|l|c|}
\hline \multicolumn{1}{|c|}{ Tipo de Predicado } & Compatibilidade com o Progressivo \\
\hline atividade & $\checkmark$ \\
\hline accomplishment & $\checkmark$ \\
\hline achievement & $x$ \\
\hline estado & $x$ \\
\hline
\end{tabular}

Em Karitiana, o auxiliar aspectual tyka é o morfema que expressa de modo mais próximo o significado do aspecto preogressivo. Tyka foi analisado como imperfectivo por Storto (2002) e Carvalho (2010) e como pregressivo em Landin, D. (1984) e Everett, C. (2006). No entanto, tyka não diferencia atividades e accomplishmentes de achievements e estados. Conforme a tabela abaixo mostra, tyka pode aparecer com todos os tipos de predicados na língua. O único predicado da tabela que não pôde ser usado com tyka foi $a k a$ Karitiana 'ser Karitiana'. Uma explicação possível para esse fenômeno pode ser o fato de esse ser um predicado individual-level canônico ${ }^{55}$. Conforme discutido anteriormente, esse é um

55 Carvalho (2010) mostrou que tyka resiste em ocorrer com predicados individual-level. Na terminologia empregada pela autora, que adota Klein, W. (1994), esses são predicados de conteúdo lexical não-temporário, que não apresentam contraste com tempo de tópico (porque são, a rigor, permanentes). Uma vez que, segundo Klein, W. (1994), esses predicados são incompativeis com marcas de imperfectivo, Carvalho (2010) utiliza essa característica como evidência de que tyka é um auxiliar aspectual imperfectivo. 
predicado que tem resistência de ser interpretado como stage-level.

Tabela 26: Teste da Compatibilidade com o Progressivo em Karitiana

\begin{tabular}{|c|c|}
\hline Verbo & Exemplo \\
\hline \multicolumn{2}{|r|}{ Atividades } \\
\hline $\begin{array}{l}\text { pykyn } \\
\text { 'correr' }\end{array}$ & $\begin{array}{l}\text { João i-pykyn-<a }>\text {-t } \quad \text { ty-ka-t. } \\
\text { João PART-correr-ABS IMP-MOV.SG-NFUT } \\
\text { 'O João está correndo' }\end{array}$ \\
\hline $\begin{array}{l}\text { pytim'adn } \\
\text { 'trabalhar' }\end{array}$ & $\begin{array}{l}\text { João i-pytim'adn-<a }>-t \quad \text { ty-ka-t. } \\
\text { João PART-trabalhar-ABS IMP-MOV.SG-NFUT } \\
\text { 'O João está trabalhando' }\end{array}$ \\
\hline $\begin{array}{l}\text { pimbik gooj } \\
\text { 'empurrar carro' }\end{array}$ & 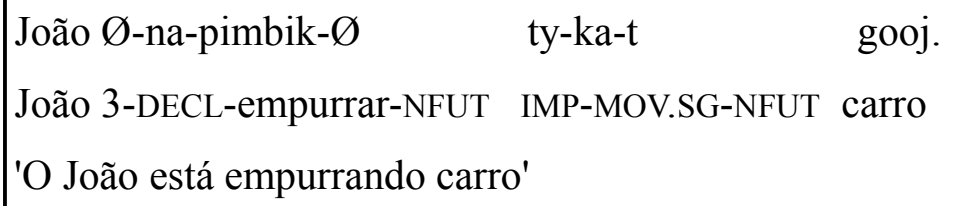 \\
\hline $\begin{array}{l}\text { tagngã gooj } \\
\text { 'dirigir carro' }\end{array}$ & $\begin{array}{ll}\text { João Ø-naka-tagngã-t } \quad \text { ty-ka-t } & \text { gooj. } \\
\text { João 3-DECL-dirigir-NFUT } & \text { IMP-MOV.SG-NFUT } \\
\text { 'O João está dirigindo o carro' } & \\
\end{array}$ \\
\hline \multicolumn{2}{|r|}{ Estativos Stage-level } \\
\hline $\begin{array}{l}\text { siki'y } \\
\text { 'querer' }\end{array}$ & $\begin{array}{l}\text { João i-siki'y-t ty-ka-t } \quad \text { ese-ty. } \\
\text { João PART-querer-ABS IMP-MOV.SG-NFUT água-OBL } \\
\text { 'O João está querendo água' }\end{array}$ \\
\hline $\begin{array}{l}\text { osedn } \\
\text { 'estar alegre' }\end{array}$ & $\begin{array}{l}\text { João i-osedn- } \varnothing \quad \text { ty-ka-t. } \\
\text { João PART-estar.alegre-ABS IMP-MOV.SG-NFUT } \\
\text { 'O João está alegre' }\end{array}$ \\
\hline $\begin{array}{l}\text { em } \\
\text { 'estar sujo' }\end{array}$ & $\begin{array}{l}\text { João naakat i-em-Ø ty-ka-t. } \\
\text { João PART-estar.sujo-ABS IMP-MOV.SG-NFUT } \\
\text { 'O João está sujo' }\end{array}$ \\
\hline $\begin{array}{l}\text { pasadn } \\
\text { 'gostar' }\end{array}$ & $\begin{array}{lll}\text { João i-pasadn-Ø } \quad \text { ty-ka-t } & \text { Milena-ty. } \\
\text { João PART-gostar-ABS } & \text { IMP-MOV.SG-NFUT } & \text { Milena-OBL } \\
\text { 'O João está gostando da Milena' } & \end{array}$ \\
\hline
\end{tabular}




\begin{tabular}{|c|c|}
\hline \multicolumn{2}{|r|}{ Estativos Individual-level } \\
\hline $\begin{array}{l}\text { aka Karitiana } \\
\text { 'ser Karitiana' }\end{array}$ & $\begin{array}{l}\text { *João Ø-na-aka-t } \quad \text { Karitiana-t } \quad \text { ty-ka-t. } \\
\text { João 3-DECL-COP-NFUT Karitiana-ABS IMP-MOV.SG-NFUT } \\
\text { 'O João é Karitiana' } \\
\text { (A sentença não é possível nem com a interpretação } \\
\text { 'O João está agindo como um Karitiana') }\end{array}$ \\
\hline $\begin{array}{l}\text { pypyyt } \\
\text { Matematicaty } \\
\text { 'saber } \\
\text { Matemática' }\end{array}$ & 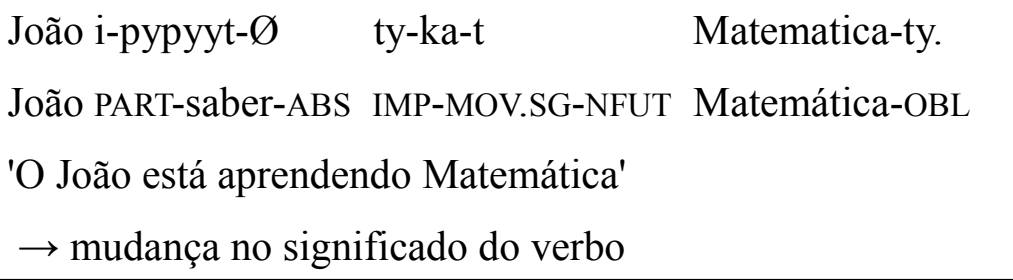 \\
\hline $\begin{array}{l}\text { kywytidn } \\
\text { Botỹjty } \\
\text { 'acreditar em } \\
\text { Deus' }\end{array}$ & $\begin{array}{l}\text { João i-kywytidn-<a>-t ty-ka-t Botỹj-ty. } \\
\text { João PART-acreditar-ABS IMP-MOV.SG-NFUT Deus-OBL } \\
\text { 'O João acredita/está acreditando em Deus' }\end{array}$ \\
\hline \multicolumn{2}{|r|}{ Accomplishment } \\
\hline $\begin{array}{l}\text { kokot Sete de } \\
\text { Setembro } \\
\text { dewota kyyn } \\
\text { 'atravessar a } \\
\text { Sete de } \\
\text { Setembro' }\end{array}$ & $\begin{array}{l}\text { João i-kokot- } \varnothing \text { ty-ka-t } \quad \text { Sete de Setembro } \\
\text { João PART-passar-ABS IMP-MOV.SG-NFUT } \\
\text { dewota kyyn. } \\
\text { outro.lado para } \\
\text { 'O João está atravessando a rua Sete de Setembro' }\end{array}$ \\
\hline $\begin{array}{l}\text { pog pyejepa } \\
\text { goot } \\
\text { 'pintar a escola } \\
\text { nova de branco' }\end{array}$ & $\begin{array}{l}\text { João } \varnothing \text {-naka-pog-<a }>\text {-t ty-ka-t } \\
\text { João 3-DECL-pintar.de.branco-NFUT IMP-MOV.SG-NFUT } \\
\text { pyejepa goot. } \\
\text { escola novo } \\
\text { 'O João está pintando a escola nova de branco' }\end{array}$ \\
\hline $\begin{array}{l}\text { tat Porto Velho } \\
\text { pip } \\
\text { 'ir para Porto } \\
\text { Velho' }\end{array}$ & $\begin{array}{lll}\text { João itat } & \text { ty-ka-t } & \text { Porto Velho pip. } \\
\text { João PART-ir-ABS } & \text { IMP-MOV.SG-NFUT } & \text { Porto Velho para } \\
\text { 'O João está indo para Porto Velho' } & \end{array}$ \\
\hline
\end{tabular}




\begin{tabular}{|c|c|}
\hline $\begin{array}{l}\text { 'a myhint boet } \\
\text { 'fazer um colar' }\end{array}$ & 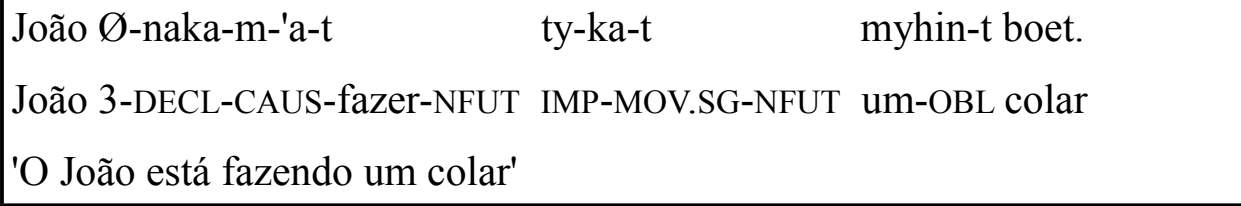 \\
\hline \multicolumn{2}{|r|}{ Achievement } \\
\hline $\begin{array}{l}\text { рор } \\
\text { 'morrer' }\end{array}$ & $\begin{array}{l}\text { João i-pop-Ø } \quad \text { ty-ka-t. } \\
\text { João PART-morrer-ABS IMP-MOV.SG-NFUT } \\
\text { 'O João está morrendo' }\end{array}$ \\
\hline $\begin{array}{l}\text { otam } \\
\text { 'chegar' }\end{array}$ & $\begin{array}{l}\text { João i-otam- } \varnothing \quad \text { ty-ka-t. } \\
\text { João PART-chegar-ABS IMP-MOV.SG-NFUT } \\
\text { 'O João está chegando' }\end{array}$ \\
\hline $\begin{array}{l}\text { typ Antônio } \\
\text { Bigode } \\
\text { 'encontrar } \\
\text { Antônio } \\
\text { Bigode' }\end{array}$ & $\begin{array}{l}\text { João Ø-naka-typ-Ø } \quad \text { ty-ka-t } \quad \text { Antônio Bigode. } \\
\text { João 3-DECL-encontrar-NFUT } \\
\text { IMP-MOV.SG-NFUT Antônio Bigode } \\
\text { 'O João está encontrando o Antônio Bigode' }\end{array}$ \\
\hline $\begin{array}{l}\text { otam myhint } \\
\text { deso ohyn sok } \\
\text { 'chegar no topo } \\
\text { de uma } \\
\text { montanha' }\end{array}$ & 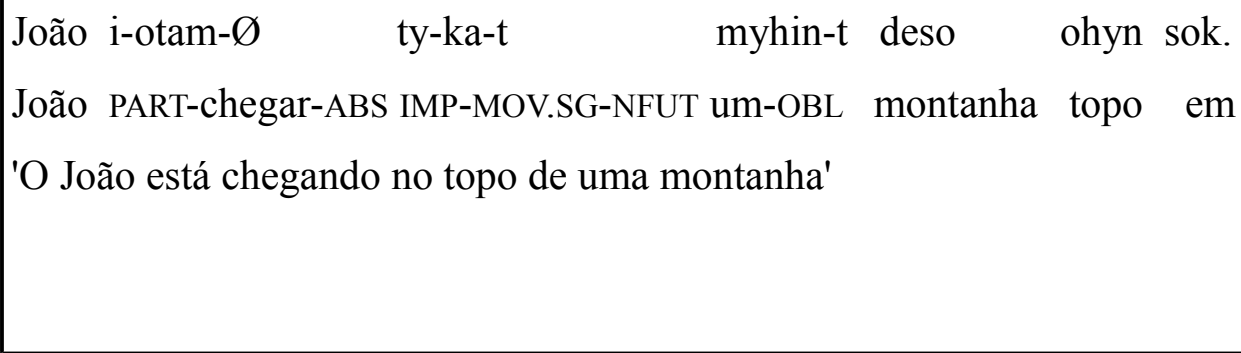 \\
\hline
\end{tabular}

Há dois testes exclusivos para separar os predicados estativos dos demais. Um deles é o da incompatibilidade de sentenças com verbos estativos e uma sentença adicional do tipo fazer o mesmo. Os exemplos abaixo mostram que o teste se aplica em português.

(143) a. O João correu e a Maria fez o mesmo.

ATIVIDADE

b. $\quad$ O João desenhou um círculo e a Maria fez o mesmo.

ACCOMPLISHMENT

c. O João reconheceu o Pedro e a Maria fez o mesmo.

ACHIEVEMENT

d. * J João soube Matemática e a Maria fez o mesmo. ESTADO 
Tabela 27: Teste da Compatibilidade com Fez o Mesmo

\begin{tabular}{|l|c|}
\hline Tipo de Predicado & Compatibilidade com Fez o Mesmo \\
\hline atividade & $\checkmark$ \\
\hline accomplishment & $\checkmark$ \\
\hline achievement & $\checkmark$ \\
\hline estado & $\times$ \\
\hline
\end{tabular}

Esse teste, no entato, não se aplica em Karitiana. Conforme apresentado na tabela 28, abaixo, todos os verbos podem ser usados em sentenças complementadas por Ahorotyym naka'at Karin 'A Karin fez também' em Karitiana. Uma das causas da inaplicabilidade do teste na língua é o fato de que o verbo ' $a$ em Karitiana parece não significar exatamento o que significa o verbo fazer em português. Normalmente, as sentenças que indicam 'fazer algo', como no caso do VP de accomplishment que estamos utilizando 'a myhint boet 'fazer um colar', contêm um morfema causativo. Nessas sentenças, 'a parece significar 'existir'. Assim, 'fazer um colar' é algo como 'causar a existência de um colar'. No entando, nas sentenças com ahorotyym naka'at Karin 'a Karin fez o mesmo', o morfema causativo não aparece. Nessas sentenças, ' $a$ parece indicar 'fazer', e não 'existir' ${ }^{56}$.

Tabela 28: Teste da Compatibilidade com o Fez o Mesmo em Karitiana

\begin{tabular}{|c|c|}
\hline Verbo & Exemplo \\
\hline \multicolumn{2}{|r|}{ Atividades } \\
\hline $\begin{array}{l}\text { pykyn } \\
\text { 'correr' }\end{array}$ & 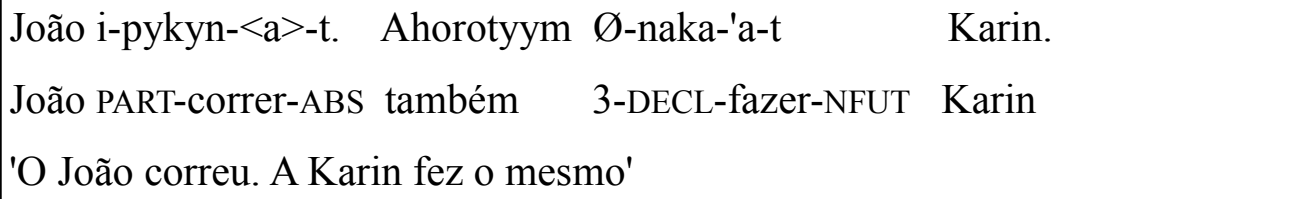 \\
\hline $\begin{array}{l}\text { pytim'adn } \\
\text { 'trabalhar' }\end{array}$ & $\begin{array}{l}\text { João i-pytim'adn-<a>-t ahorotyym } \varnothing \text {-naka-'a-t } \quad \text { Karin. } \\
\text { João PART-trabalhar-ABS também } \quad 3 \text {-DECL-fazer-NFUT Karin } \\
\text { 'O João trabalhou, a Karin fez o mesmo' }\end{array}$ \\
\hline $\begin{array}{l}\text { pimbik gooj } \\
\text { 'empurrar } \\
\text { carro' }\end{array}$ & $\begin{array}{llll}\text { João Ø-na-pimbik-Ø } & \text { gooj. Ahorotyym } & \varnothing \text {-naka-'a-t } & \text { Karin. } \\
\text { João 3-DECL-empurrar-NFUT } & \text { carro também } & \text { 3-DECL-fazer-NFUT } & \text { Karin } \\
\text { 'O João empurrou carro, a Karin fez o mesmo' } & \end{array}$ \\
\hline
\end{tabular}

56 Investigar qual o significado de ' $a$ é um trabalho ainda a ser feito. 


\begin{tabular}{|c|c|}
\hline $\begin{array}{l}\text { tagngã gooj } \\
\text { 'dirigir carro' }\end{array}$ & 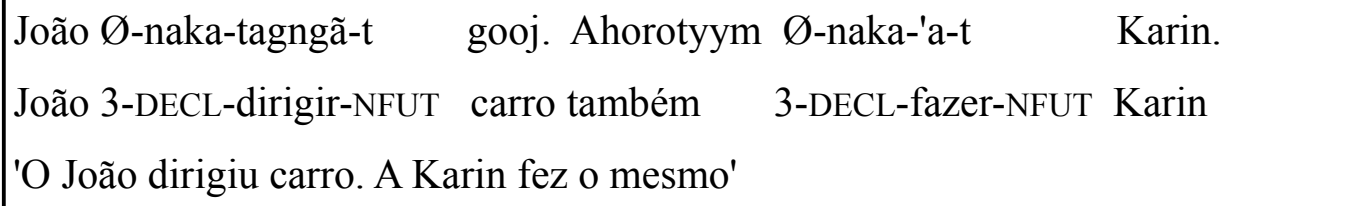 \\
\hline \multicolumn{2}{|r|}{ Estativos Stage-level } \\
\hline $\begin{array}{l}\text { siki'y } \\
\text { 'querer' }\end{array}$ & 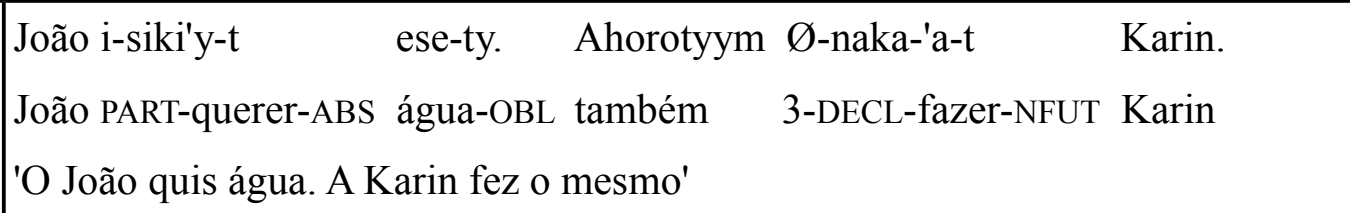 \\
\hline $\begin{array}{l}\text { osedn } \\
\text { 'estar alegre' }\end{array}$ & 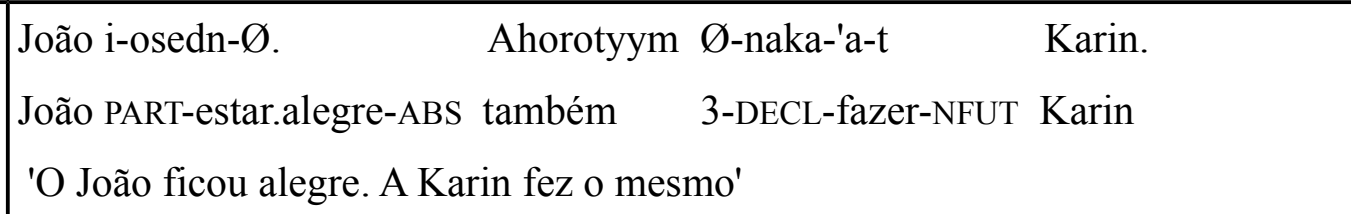 \\
\hline $\begin{array}{l}\text { em } \\
\text { 'estar sujo' }\end{array}$ & 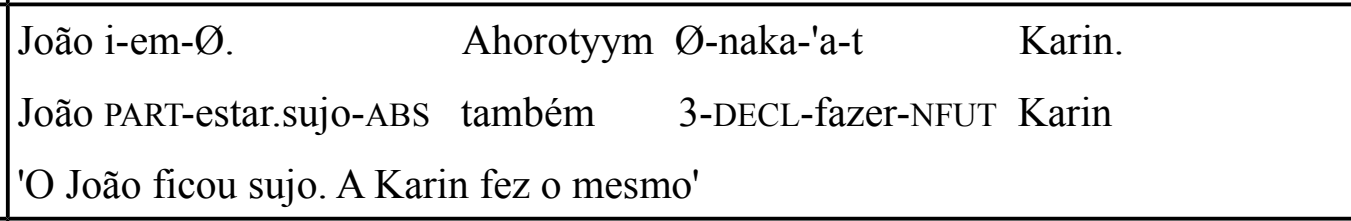 \\
\hline $\begin{array}{l}\text { pasadn } \\
\text { 'gostar' }\end{array}$ & $\begin{array}{lll}\text { João i-pasadn- } \varnothing & \text { Milena-ty. } & \text { Ahorotyym } \\
\text { João PART-gostar-ABS } & \text { Milena-OBL } & \text { também } \\
\text { Ø-naka-'a-t } & \text { Karin. } \\
\text { 3-DECL-fazer-NFUT } & \text { Karin } \\
\text { 'O João gosta/gostou da Milena. A Karin fez o mesmo' }\end{array}$ \\
\hline \multicolumn{2}{|r|}{ Estativos Individual-level } \\
\hline $\begin{array}{l}\text { aka } \\
\text { Karitiana } \\
\text { 'ser Karitiana' }\end{array}$ & 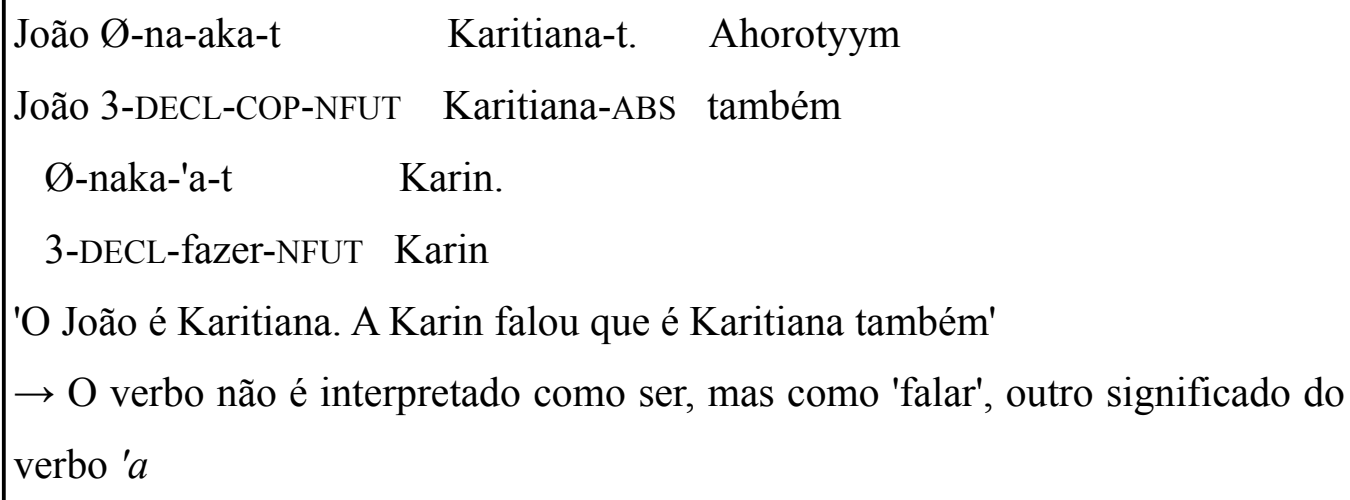 \\
\hline
\end{tabular}




\begin{tabular}{|c|c|}
\hline $\begin{array}{l}\text { pypyyt } \\
\text { Matematicat } \\
y \\
\text { 'saber } \\
\text { Matemática' }\end{array}$ & $\begin{array}{l}\text { João i-pypyyt- } \quad \text { Matematica-ty. Ahorotyym } \\
\text { João PART-saber-ABS Matemática-OBL também } \\
\text { Ø-naka-'a-t } \quad \text { Karin. } \\
\text { 3-DECL-fazer-NFUT Karin } \\
\text { 'O João aprendeu Matemática. A Karin fez o mesmo' } \\
\rightarrow \text { mudança no significado do verbo }\end{array}$ \\
\hline $\begin{array}{l}\text { kywytidn } \\
\text { Botyjjty } \\
\text { 'acreditar em } \\
\text { Deus' }\end{array}$ & $\begin{array}{l}\text { João i-kywytidn-<a>-t Botỹj-ty. Ahorotyym Ø-naka-'a-t } \\
\text { João PART-acreditar-ABS Deus-OBL também 3-DECL-fazer-NFUT Karin } \\
\text { 'O João acreditou em Deus. A Karin fez o mesmo' }\end{array}$ \\
\hline & Accomplishment \\
\hline $\begin{array}{l}\text { kokot Sete de } \\
\text { Setembro } \\
\text { dewota kyyn } \\
\text { 'atravessar a } \\
\text { Sete de } \\
\text { Setembro' }\end{array}$ & 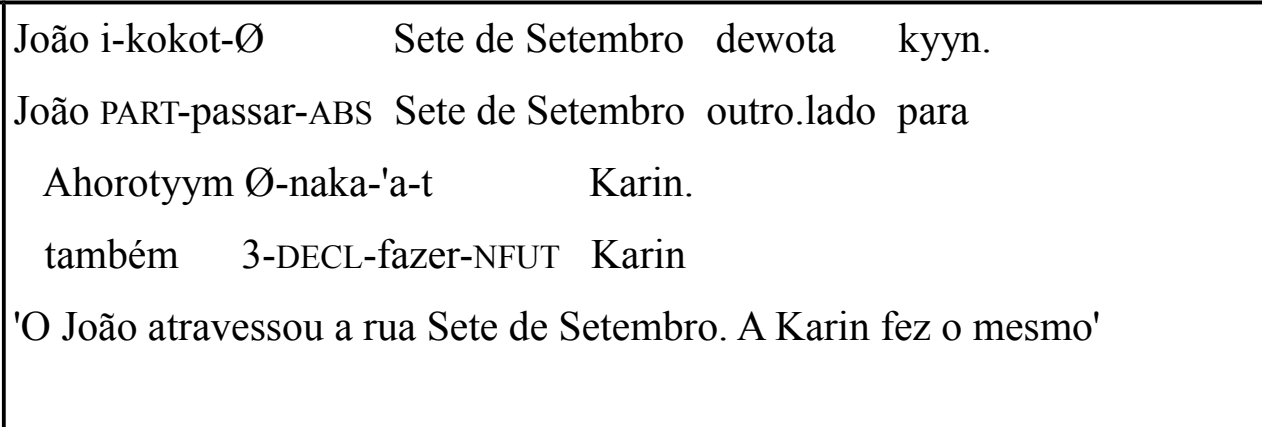 \\
\hline $\begin{array}{l}\text { pog pyejepa } \\
\text { goot } \\
\text { 'pintar a } \\
\text { escola nova } \\
\text { de branco' }\end{array}$ & $\begin{array}{l}\text { João } \varnothing \text {-naka-pog-<a>-t } \quad \text { pyejepa goot. Ahorotyym } \\
\text { João 3-DECL-pintar.de.branco-NFUT } \\
\text { Ø-naka-'a-t } \quad \text { Karin. } \\
\text { 3-DECL-fazer-NFUT Karin } \\
\text { 'O João pintou a escola nova de branco. A Karin fez o mesmo' }\end{array}$ \\
\hline $\begin{array}{l}\text { tat Porto } \\
\text { Velho pip } \\
\text { 'ir para Porto } \\
\text { Velho' }\end{array}$ & $\begin{array}{l}\text { João itat Porto Velho pip. Ahorotyym } \varnothing \text {-naka-'a-t } \\
\text { João PART-ir-ABS Porto Velho para também } \\
\text { 3-DECL-fazer-NFUT }\end{array}$ \\
\hline $\begin{array}{l}\text { 'a myhint } \\
\text { boet } \\
\text { 'fazer um } \\
\text { colar' }\end{array}$ & 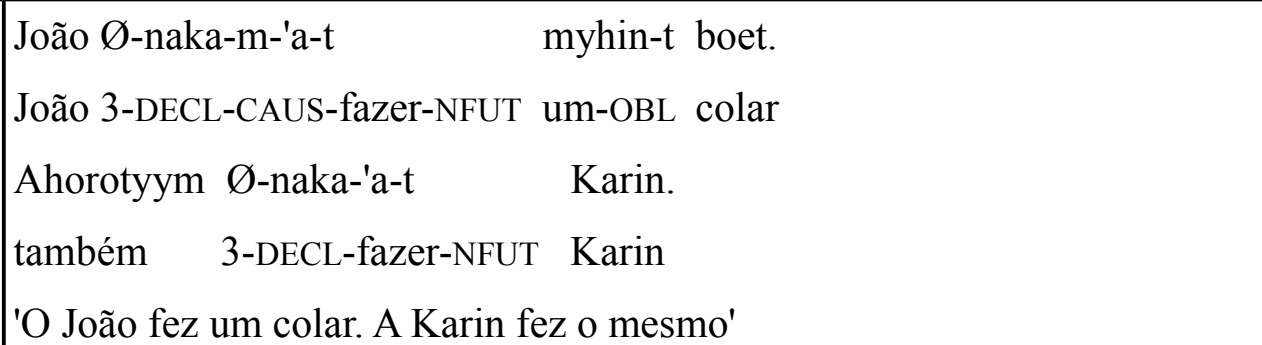 \\
\hline
\end{tabular}




\begin{tabular}{|c|c|}
\hline \multicolumn{2}{|r|}{ Achievement } \\
\hline $\begin{array}{l}\text { рор } \\
\text { 'morrer' }\end{array}$ & 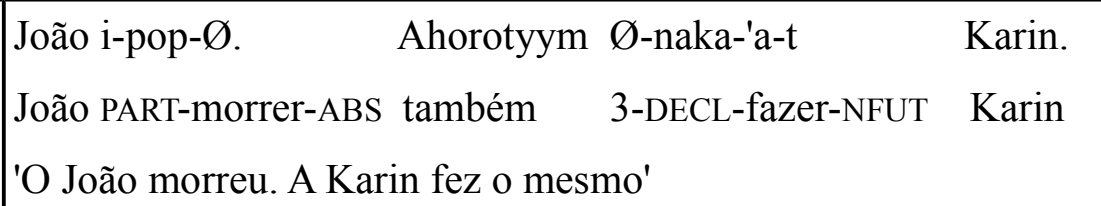 \\
\hline $\begin{array}{l}\text { otam } \\
\text { 'chegar' }\end{array}$ & $\begin{array}{lll}\text { João i-otam- } \varnothing . & \text { Ahorotyym } \varnothing \text {-naka-'a-t } & \text { Karin. } \\
\text { João PART-chegar-ABS } & \text { também } \quad \text { 3-DECL-fazer-NFUT } & \text { Karin } \\
\text { 'O João chegou. A Karin fez o mesmo' } & \end{array}$ \\
\hline $\begin{array}{l}\text { typ Antônio } \\
\text { Bigode } \\
\text { 'encontrar } \\
\text { Antônio } \\
\text { Bigode' }\end{array}$ & 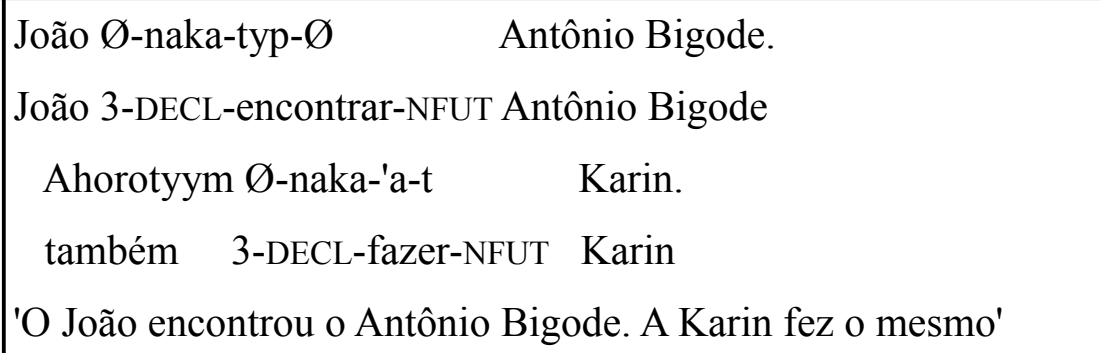 \\
\hline $\begin{array}{l}\text { otam myhint } \\
\text { deso ohyn } \\
\text { sok } \\
\text { 'chegar no } \\
\text { topo de uma } \\
\text { montanha' }\end{array}$ & $\begin{array}{lll}\text { João i-otam- } \varnothing \quad \text { myhin-t } & \text { deso } & \text { ohyn sok. } \\
\text { João PART-chegar-ABS um-OBL } & \text { montanha topo em } \\
\text { Ahorotyym } \varnothing \text {-naka-'a-t } & \text { Karin. } \\
\text { também 3-DECL-fazer-NFUT Karin } & \text { Ko } \\
\text { 'O João chegou no topo de uma montanha. A Karin fez o mesmo' }\end{array}$ \\
\hline
\end{tabular}

O segundo teste usado para separar estativos dos outros predicados diz respeito à incompatibilidade com o modo imperativo. As sentenças abaixo ilustram essa impossibilidade, que se aplica em português.
a. Corra, João!
b. Desenhe um círculo, João!
c. Alcance o topo da montanha, João!
d. * Saiba Matemática, João!

ATIVIDADE

ACCOMPLISHMENT

ACHIEVEMENT

ESTADO 
Tabela 29: Teste da Compatibilidade com o Imperativo

\begin{tabular}{|l|c|}
\hline Tipo de Predicado & Compatibilidade com o Imperativo \\
\hline atividade & $\checkmark$ \\
\hline accomplishment & $\checkmark$ \\
\hline achievement & $\checkmark$ \\
\hline estado & $\times$ \\
\hline
\end{tabular}

Mais uma vez, o teste não se aplica em Karitana. Todos os tipos de predicado verbal da língua podem ocorrer na construção de modo imperativo. O modo imperativo na língua é construído da seguinte forma: PREFIXO DE PESSOA-VERBO-SUFIXO DE MODO IMPERATIVO. Os prefixos de concordância de pessoa são distintos dos apresentados no capítulo 1, que fícou focado em apresentar as características gerais da língua a partir de sentenças no modo declarativo. A tabela abaixo apresenta os morfemas de pessoa em sentenças não declarativas. Estão presentes apenas a segunda e a terceira pessoa porque são os morfemas relevantes para os dados apresentados com o imperativo ${ }^{57}$.

Tabela 30: Prefixos de Concordância de Pessoa em Sentenças não Declarativas

\begin{tabular}{|l|c|}
\hline \multicolumn{1}{|c|}{ PESSOA } & MORFEMA \\
\hline $2^{\mathrm{a}} \mathrm{SG}$ & $\{a-\}$ \\
\hline $3^{\mathrm{a}}$ pessoa (SG ou PL) & $\{i-\}$ \\
\hline
\end{tabular}

Os sufixos de modo imperativo são $\{-a\}$ se verbo terminar com consoante, ou $\{-\emptyset\}$ se o verbo terminar com vogal.

\footnotetext{
$\overline{57}$ Para mais detalhes sobre os morfemas de concordância na língua, ver Storto (2002).
} 
Tabela 31: Teste da Compatibilidade com o Imperativo em Karitiana

\begin{tabular}{|c|c|}
\hline Verbo & Exemplo \\
\hline \multicolumn{2}{|r|}{ Atividades } \\
\hline $\begin{array}{l}\text { pykyn } \\
\text { 'correr' }\end{array}$ & $\begin{array}{l}\text { A-pykyn-a João. } \\
\text { 2s-correr-IMPR João } \\
\text { 'Corra, João!' }\end{array}$ \\
\hline $\begin{array}{l}\text { pytimadn } \\
\text { 'trabalhar' }\end{array}$ & $\begin{array}{l}\text { A-pytim'adn-a João. } \\
\text { 2s-trabalhar-IMPR João } \\
\text { 'Trabalhe, João!' }\end{array}$ \\
\hline $\begin{array}{l}\text { pimbik gooj } \\
\text { 'empurrar carro' }\end{array}$ & $\begin{array}{l}\text { I-pimbik-a gooj João. } \\
\text { 3-empurrar-IMPR carro João } \\
\text { 'Empurre o carro, João!' }\end{array}$ \\
\hline $\begin{array}{l}\text { tagngã gooj } \\
\text { 'dirigir carro' }\end{array}$ & $\begin{array}{l}\text { I-tagngã-Ø gooj João. } \\
\text { 3-dirigir-IMPR carro João } \\
\text { 'Dirija o carro, João!' }\end{array}$ \\
\hline \multicolumn{2}{|r|}{ Estativos Stage-level } \\
\hline $\begin{array}{l}\text { siki'y } \\
\text { 'querer' }\end{array}$ & $\begin{array}{l}\text { A-siki'y-Ø ese-ty João. } \\
\text { 2s-querer-IMPR água-OBL João } \\
\text { 'Queira água, João!' }\end{array}$ \\
\hline $\begin{array}{l}\text { osedn } \\
\text { 'estar alegre' }\end{array}$ & $\begin{array}{l}\text { A-osedn-a João. } \\
\text { 2s-estar.alegre-IMPR João } \\
\text { 'Fique feliz/alegre-se, João!' }\end{array}$ \\
\hline $\begin{array}{l}\text { em } \\
\text { 'estar sujo' }\end{array}$ & 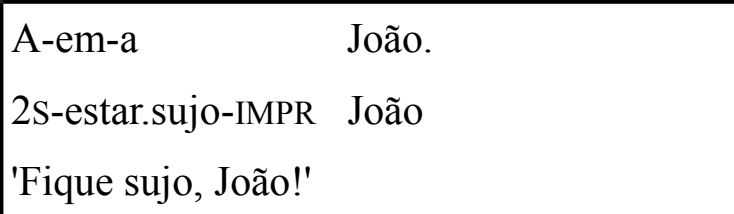 \\
\hline $\begin{array}{l}\text { pasadn } \\
\text { 'gostar' }\end{array}$ & $\begin{array}{l}\text { A-pasadn-a } \quad \text { Milena-ty } \quad \text { João. } \\
\text { 2s-gostar-IMPR } \quad \text { Milena-OBL João } \\
\text { 'Goste da Milena, João!' }\end{array}$ \\
\hline \multicolumn{2}{|r|}{ Estativos Individual-level } \\
\hline $\begin{array}{l}\text { aka Karitiana } \\
\text { 'ser Karitiana' }\end{array}$ & $\begin{array}{l}\text { A-aka-Ø Karitiana João. } \\
\text { 2S-ser-IMPR Karitiana João } \\
\text { 'Seja Karitiana, João!' }\end{array}$ \\
\hline
\end{tabular}




\begin{tabular}{|c|c|}
\hline $\begin{array}{l}\text { pypyyt } \\
\text { Matematicaty } \\
\text { 'saber } \\
\text { Matemática' }\end{array}$ & $\begin{array}{l}\text { A-pypyyt-Ø Matematica João. } \\
\text { 2s-saber-IMPR Matemática João } \\
\text { 'Aprenda Matemática, João!' } \\
\rightarrow \text { mudança no significado do verbo }\end{array}$ \\
\hline $\begin{array}{l}\text { kywytidn Botỹj } \\
\text { Botỹjy } \\
\text { 'acreditar em } \\
\text { Deus' }\end{array}$ & $\begin{array}{lll}\text { A-kywytidn-a } \quad \text { Botỹj-ty } & \text { João. } \\
\text { 2s-acreditar-IMPR } & \text { Deus-OBL } & \text { João } \\
\text { 'Acredite em Deus, João!' } & \end{array}$ \\
\hline & Accomplishment \\
\hline $\begin{array}{l}\text { kokot Sete de } \\
\text { Setembro dewota } \\
\text { kyyn } \\
\text { 'atravessar a Sete } \\
\text { de Setembro' }\end{array}$ & $\begin{array}{ll}\text { A-kokot-a } \quad \text { Sete de Setembro dewota kyyn João. } \\
\text { 2s-passar-IMPR Sete de Setembro outro.lado para João } \\
\text { 'Atravesse a rua Sete de Setembro, João!' }\end{array}$ \\
\hline $\begin{array}{l}\text { pog pyejepa goot } \\
\text { 'pintar a escola } \\
\text { nova de branco' }\end{array}$ & $\begin{array}{l}\text { I-pog-a pyejepa goot João. } \\
\text { 3S-pintar.de.branco-IMPR escola novo João } \\
\text { 'Pinte a escola nova de branco, João!' } \\
\end{array}$ \\
\hline $\begin{array}{lll}\text { tat } & \text { Porto } & \text { Velho } \\
\text { pip } & & \\
\text { 'ir para Porto } \\
\text { Velho' }\end{array}$ & $\begin{array}{l}\text { A-tat-a Porto Velho pip João. } \\
\text { 2S-ir-IMPR Porto Velho para João } \\
\text { 'Vá pra Porto Velho, João!' }\end{array}$ \\
\hline $\begin{array}{l}\text { 'a myhint boet } \\
\text { 'fazer um colar' }\end{array}$ & $\begin{array}{lll}\text { I-m-'a-Ø } & \text { myhin-t boet-<o }> & \text { João. } \\
\text { 3-CAUS-fazer-IMPR } & \text { um-OBL colar } & \text { João } \\
\text { 'Faça um colar, João!' } & \\
\end{array}$ \\
\hline & Achievement \\
\hline $\begin{array}{l}\text { рор } \\
\text { 'morrer' }\end{array}$ & $\begin{array}{ll}\text { A-pop-a } \quad \text { bykypa. } \\
\text { 2S-morrer-IMPR barata } \\
\text { 'Morra, barata!' }\end{array}$ \\
\hline $\begin{array}{l}\text { otam } \\
\text { 'chegar' }\end{array}$ & $\begin{array}{l}\text { A-otam-a João. } \\
\text { 2S-chegar-IMPR João } \\
\text { 'Chegue, João!' }\end{array}$ \\
\hline
\end{tabular}




\begin{tabular}{|l|l|}
\hline typ Antônio & I-typ-a \\
Bigode & 3-encontrar-IMPR Antônio Bigode João \\
'encontrar & 'Encontre o Antônio Bigode, João!' \\
Antônio Bigode' & \\
\hline otam myhint & A-otam-a myhin-t deso ohyn sok João. \\
deso ohyn sok & 2S-chegar-IMPR um-OBL montanha topo em João \\
chegar no topo & 'Chegue no topo de uma montanha, João!' \\
de uma & \\
montanha' & \\
\hline
\end{tabular}

Um outro teste para distinguir predicados télicos de atélicos é o que diz respeito à ambiguidade com quase. As sentenças em (145) ilustram esse teste. A sentença (145a) tem apenas a leitura de que o João ia correr, mas nem começou a correr, ou seja, ele não correu. Já (145b) é ambígua. A sentença pode ser usada para descrever um cenário em que o João ia desenhar um círculo, mas nem começou a desenhar. Mas, (145b) pode ser usada também em uma situação em que o João começou a desenhar um círculo, mas interrompeu a ação antes de terminar.

(145) a. O João quase correu.

b. O João quase desenhou um círculo.

Tabela 32: Teste da Ambiguidade com Quase

\begin{tabular}{|l|c|}
\hline Tipo de Predicado & Ambíguo com Quase \\
\hline télico & $\checkmark$ \\
\hline atélico & $x$ \\
\hline
\end{tabular}

Esse teste foi adaptado para ser aplicado em Karitiana. Aparentemente, não há na língua uma estratégia para se expressar a noção de 'quase'. No entanto, o sufixo $\{-w a k\}$ tem um comportamento semelhante ao de quase do português. \{-wak\} significa, contudo, 'querer', 'estar com vontade de ${ }^{158}$. Mas, assim como o quase, ele representa também uma ambiguidade.

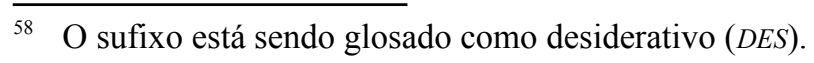


Com predicados atélicos, ele não é ambíguo. A sentença (146a) só pode ser usada em um contexto em que o João não correu. Com predicados télicos, por outro lado, a sentença é ambígua entre uma interpretação de que o João quer começar a pintar a escola e uma situação em que o João já está pintando a escola e quer terminar o evento.

$$
\begin{aligned}
& \text { a. João Ø-na-aka-t i-pykyn- }<\mathrm{i}>\text {-wak } \\
& \text { João 3-DECL-COP-NFUT PART-correr-DES } \\
& \text { 'O João quer correr' } \\
& \text { Situações: } \checkmark \text { quer começar a correr } \\
& \text { × já começou a correr }
\end{aligned}
$$

b. João Ø-naka-pog-<i $>$-wak pyejepa goot.

João 3-DECL-pintar.de.branco-DES escola novo

'O João quer pintar a escola nova de branco'

$$
\begin{aligned}
\text { Situações: } & \checkmark \quad \text { quer começar a pintar } \\
& \checkmark \quad \text { já começou a pintar/quer terminar de pintar }
\end{aligned}
$$

A tabela 33, abaixo, mostra exemplos com a tabela de verbos e ambiguidade com $\{-w a k\}$. O teste é aplicado a predicados de atividade a accomplishments. 
Tabela 33: Teste da Ambiguidade com Quase em Karitiana

\begin{tabular}{|c|c|}
\hline Verbo & Exemplo \\
\hline \multicolumn{2}{|r|}{ Atividades } \\
\hline $\begin{array}{l}\text { pykyn } \\
\text { 'correr' }\end{array}$ & 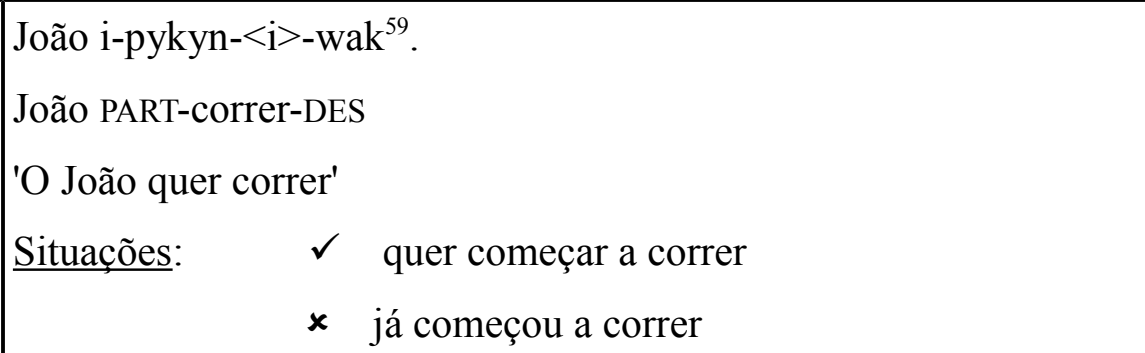 \\
\hline $\begin{array}{l}\text { pytim'adn } \\
\text { 'trabalhar' }\end{array}$ & 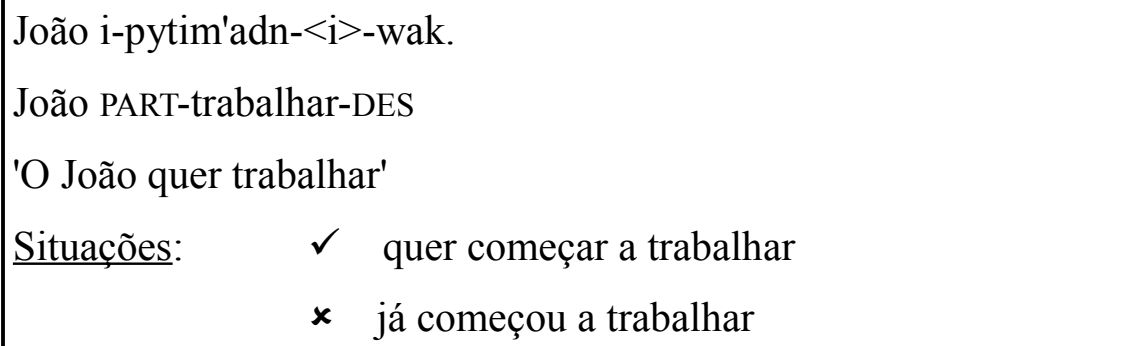 \\
\hline $\begin{array}{l}\text { pimbik gooj } \\
\text { 'empurrar carro' }\end{array}$ & 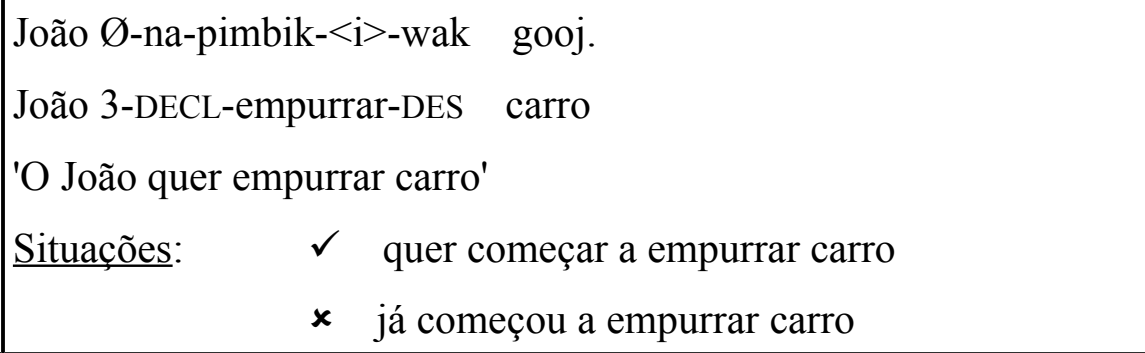 \\
\hline $\begin{array}{l}\text { tagngã gooj } \\
\text { 'dirigir carro' }\end{array}$ & $\begin{array}{l}\text { João Ø-naka-tagngã-wak gooj. } \\
\text { João 3-DECL-dirigir-DES carro } \\
\text { 'O João quer dirigir carro' } \\
\begin{array}{rlr}\text { Situações: } & \checkmark & \text { quer começar a dirigir carro } \\
& \times & \text { já começou a dirigir carro }\end{array}\end{array}$ \\
\hline \multicolumn{2}{|r|}{ Accomplishment } \\
\hline $\begin{array}{l}\text { kokot Sete de } \\
\text { Setembro } \\
\text { dewota kyyn }\end{array}$ & $\begin{array}{l}\text { João i-kokot- }<\text { o }>\text {-wak Sete de Setembro dewota kyyn. } \\
\text { João PART-passar-DES Sete de Setembro outro.lado para } \\
\text { 'O João quer atravessar a rua Sete de Setembro' }\end{array}$ \\
\hline $\begin{array}{l}\text { 'atravessar a } \\
\text { Sete de } \\
\text { Setembro' }\end{array}$ & 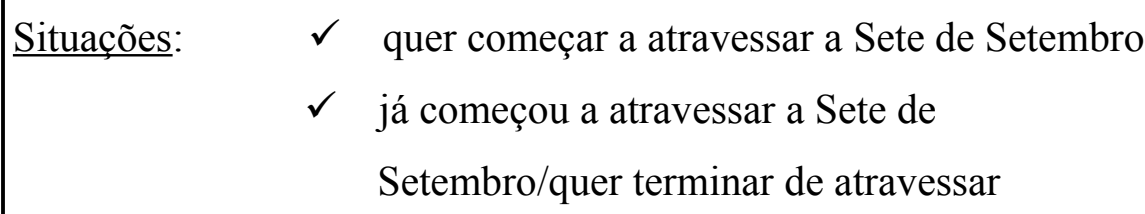 \\
\hline
\end{tabular}

59 Quando o morfema $\{-w a k\}$ é sufixado e um verbo que termina em consoante, uma vogal epentética é inserida. 


\begin{tabular}{|c|c|}
\hline $\begin{array}{l}\text { pog pyejepa } \\
\text { goot } \\
\text { 'pintar a escola } \\
\text { nova de branco' }\end{array}$ & 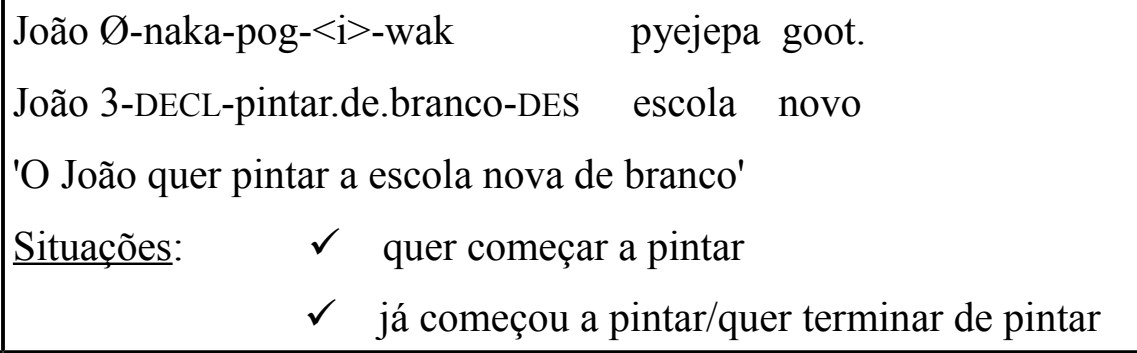 \\
\hline $\begin{array}{l}\text { tat Porto Velho } \\
\text { pip } \\
\text { 'ir para Porto } \\
\text { Velho' }\end{array}$ & 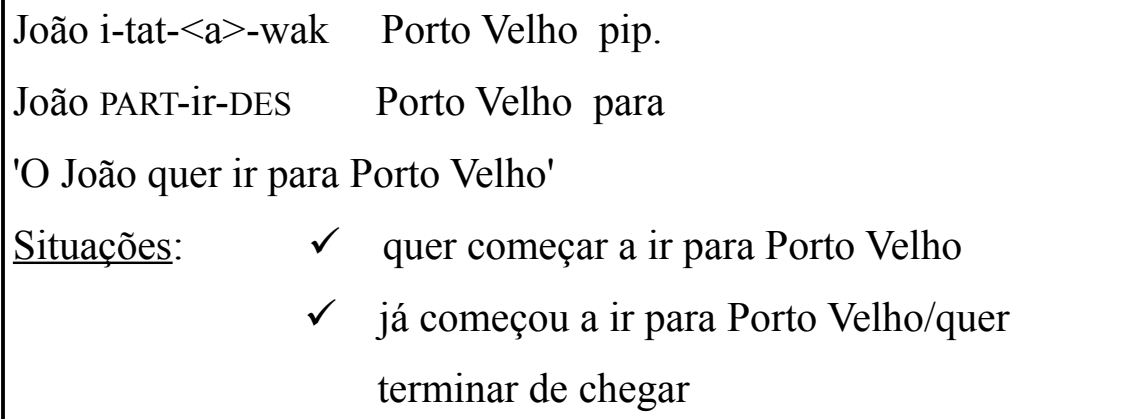 \\
\hline $\begin{array}{l}\text { 'a myhint boet } \\
\text { 'fazer um colar' }\end{array}$ & 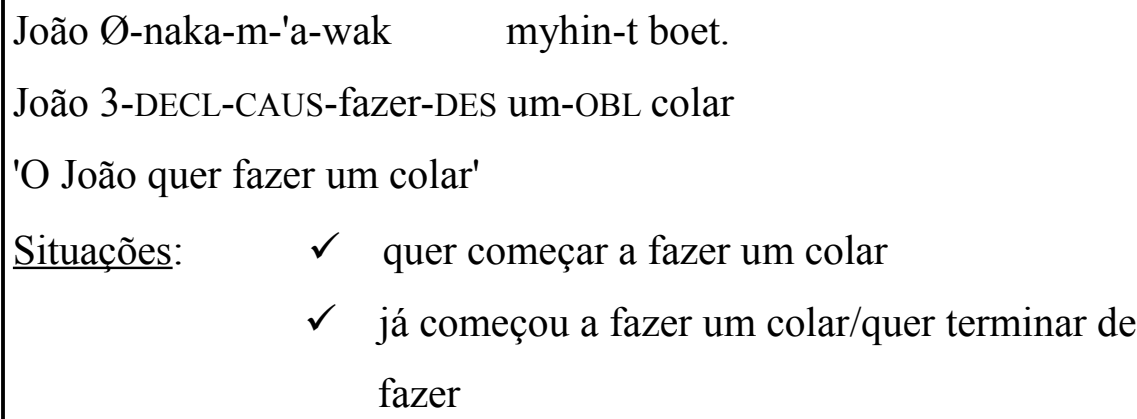 \\
\hline
\end{tabular}

Esta seção mostrou os resultados da aplicação de testes clássicos para a distinção em Karitiana das classes verbais. Vimos que, no geral, os testes precisam ser adaptados para funcionarem na língua.

O primeiro teste analisado foi o da distribuição dos adjuntos por $X$ tempo e em $X$ tempo. Em Karitiana, o teste não se aplica por não haver duas posposições que expressem essas noções. O segundo teste discutido foi o do paradoxo do imperfectivo. Vimos que o fato de que a língua parece não possuir uma estratégia para expressar o perfectivo interfere na aplicação do teste. Foi necessária a elicitação de contextos e a inserção de sentenças do tipo 'e não terminou de'. Com a adaptação do teste, foi possível notar que há uma diferença entre predicados télicos e atélicos na língua.

Um outro teste que funcionou em Karitiana foi o da ambiguidade com 'quase'. O teste foi adaptado com o sufixo desiderativo $\{-w a k\}$. A ambiguidade das sentenças com verbos télicos e esse sufixo mostraram que a telicidade é um traço que distingue as classes 
verbais em Karitiana.

Os testes da compatibilidade com o progressivo, com a sentença ahorotyym naka'at Karin 'a Karin fez o mesmo' e da compatibilidade com o imperativo não se aplicam em Karitiana. Esse fato indica apenas que os testes foram elaborados para outras línguas, e não necessariamente que a língua Karitiana é muito distinta no que diz respeito às classes acionais. Esses testes são discutíveis também quando aplicados, por exemplo, em português brasileiro. O que a seção mostrou foi que para se discutir as classes acionais em uma nova língua é preciso considerar suas características particulares e reelaborar os testes clássicos apresentados pela literatura.

Não foi encontrado nenhum teste que determinasse que estados stage-level $\mathrm{e}$ atividades são diferentes, bem como que distinguisse accomplishments de achievements ${ }^{60}$. Embora essas sejam distinções que não tenham sido comprovadas, ela vai continuar fazendo parte da tabela de verbos que vai ser retomada em outros pontos desta tese. Essa escolha foi feita apenas para manter uma homoneidade com as classes que são normalmente discutidas na literatura. Essas distinções não serão relevantes para a análise defendida nesta tese. Apenas a distinção télico-atélico, que foi comprovada nesta seção, será importante para a análise apresentada no próximo capítulo.

\subsection{Considerações Finais}

Esta seção problematizou a classificação dos predicados verbais em Karitiana segundo a sua acionalidade. A primeira parte da seção mostrou algumas teorias influentes sobre acionalidade. Vendler (1957) foi o primeiro a sistematizar os verbos segundo classes acionais. Sua abordagem está diretamente relacionada com o comportamento dos verbos em relação ao tempo. Verkuyl (1996) critica essa versão temporal e sugere uma abordagem composicional, em que é de suma importância a participação dos sintagmas nominais para a classificação. Krifka (1998) segue a proposta de Verkuyl (1996) de que o predicado verbal como um todo, junto com seu objeto deve ser considerado para a avaliação. Krifka (1998), no entanto, propõe uma análise mereológica para a telicidade que leva em conta a existência de um homomorfismo entre as estruturas dos indivíduos e dos eventos. Rothstein (2004), por sua

60 Os predicados individual-level se comportaram como predicados stage-level em alguns testes. Apenas o predicado aka Karitiana 'ser Karitiana' resistiu a ter uma interpretações stage-level. Isso indica que a língua possuiria predicados individual-level. A mudança de individual-level para stage-level dos outros predicados é prevista pela literatura conforme discutido anteriormente. 
vez, analisa os predicados verbais como predicados que são sempre contáveis em que o critério de atomicidade determina a telicidade. Predicados atômicos são télicos e predicados cuja atomicidade é preenchida contextualmente são atélicos.

Os dados do Karitiana foram debatidos com a teoria presentada. As propriedades dos sintagmas nominais e das marcas de tempo e aspecto foram retomadas para problematizar os dados da língua. Lenvando-se em conta essas variáveis, uma lista de verbos foi proposta e submetiida a uma gama de testes que são tradicionais na literatura sobre o tema. Os testes mostraram que os diagnósticos mais utilizados não se aplicam totalmente em Karitiana. No entanto, a adaptação de alguns testes indicou que a distinção télico-atélico é pertinente na língua. 
O foco desta seção é discutir duas propriedades semânticas do domínio verbal da língua Karitiana: a cumulatividade e a contabilidade. O objetivo é demonstrar que os verbos nessa língua têm denotação cumulativa e contável, embora essas duas propriedades pareçam incompatíveis. A aparente incompatibilidade vem da associação entre a propriedade da cumulatividade e os predicados massivos.

Essa investigação é relevante porque contribui para uma melhor compreensão da semântica dos verbos em Karitiana, o que auxilia na investigação das operações de quantidade nesse domínio, como a modificação de grau que será tema do próximo capítulo. Parte dos argumentos deste capítulo é baseada na distinção entre advérbios de frequência e de grau em Karitiana. Essa distinção sugere algumas características da modificação de grau que será explorada em seguida.

Para argumentar que o domínio verbal na língua Karitiana é cumulativo e contável, esta seção está dividida em duas partes. Na primeira subseção, são apresentados os dados da língua Karitiana que apoiam uma análise que leve em conta tanto a cumulatividade quanto a contabilidade. Mais especificamente são discutidas a operação da pluracionalidade e a distinção entre advérbios de frequência e de grau na língua. A segunda parte apresenta uma discussão teórica com o objetivo de mostrar que essas duas propriedades são compatíveis, a despeito da sua aparente contradição.

\subsection{Dados do Karitiana}

\subsubsection{Cumulatividade}

O objetivo desta seção é mostrar que a investigação de sentenças com operação de pluracionalidade em Karitiana apoia a proposta de que o domínio verbal da língua tem denotação cumulativa. A cumulatividade foi discutida na seção anterior sobre a proposta de Krifka (1998) para a telicidade. Sua definição está repetida em (147): 
(147) Cumulatividade:

$\forall \mathrm{X} \subseteq \mathrm{U}_{\mathrm{P}}\left[\mathrm{CUM}_{\mathrm{P}}(\mathrm{X}) \leftrightarrow \exists \mathrm{x}, \mathrm{y}[\mathrm{X}(\mathrm{x}) \wedge \mathrm{X}(\mathrm{y}) \wedge \neg \mathrm{x}=\mathrm{y}] \wedge \forall \mathrm{x}, \mathrm{y}[\mathrm{X}(\mathrm{x}) \wedge \mathrm{X}(\mathrm{y}) \rightarrow\right.$ $\mathrm{X}(\mathrm{x} \oplus \mathrm{P} \mathrm{y})]]$

Um predicado $X$ é cumulativo se, e somente se, no caso de existirem dois argumentos diferentes $\mathrm{x}$ e $\mathrm{y}$ aos quais o predicado $\mathrm{X}$ se aplica, então o predicado $\mathrm{X}$ também se aplica à soma de $\mathrm{x}$ e $\mathrm{y}$.

(definição 3 de Krifka 1998, p. 3)

Müller e Sanchez-Mendes (2008) afirmam que os verbos em Karitiana denotam predicados cumulativos. A propriedade foi utilizada pelas autoras para caracterizar a denotação de número neutro dos sintagmas verbais da língua. É importante notar que a propriedade da cumulatividade se aplica tanto a predicados neutros para número quanto a predicados plurais que não incluem átomos. Na proposta de Müller e Sanchez-Mendes (2008), no entanto, a propriedade foi utilizada pelas autoras para caracterizar a denotação de número neutro com base na hipótese que ficou conhecida na literatura como o Universal da Cumulatividade. O Universal da Cumulatividade postula que todos os predicados simples das línguas naturais possuem denotação cumulativa (Krifka 1998, Kratzer 2003, 2007).

Em sentenças simples, em Karitiana, o verbo pode fazer referência a um evento singular ou a uma pluralidade de eventos. O exemplo (148) com o verbo 'ot 'cair' mostra que a sentença pode ser usada tanto em uma situação em que o João caiu uma vez ou em uma situação em que o João caiu mais de uma vez.

(148) João i-'ot-Ø.

João PART-cair-ABS

'O João caiu'

$\begin{array}{lll}\text { Situações: } & \checkmark & \text { uma vez } \\ & \checkmark & \text { mais de uma vez }\end{array}$

O exemplo abaixo ilustra a cumulatividade verbal em uma sentença com verbo transitivo. A sentença é apropriada para descrever um contexto em que houve um evento apenas de Inácio levantar Nadia ou um contexto em que houve mais de um evento. 
Inácio Ø-na-mangat-Ø Nadia.

Inácio 3-DECL-levantar-NFUT Nadia

'O Inácio levantou a Nadia'

Situações: $\checkmark$ uma vez

$\checkmark \quad$ mais de uma vez

As sentenças (148) e (149) mostram que os sintagmas verbais simples têm uma denotação neutra para número em Karitiana. Eles podem ser usados para expressar um evento singular ou uma pluralidade de eventos.

Isso não quer dizer que em todas as sentenças o predicado verbal terá número neutro. A neutralidade para número não é mantida em sentenças que apresentam morfemas que expressam pluracionalidade. A pluracionalidade é a marcação de eventos plurais por meio de afixos verbais, chamados de marcadores pluracionais (Lasersohn 1995). Os marcadores pluracionais são morfemas verbais que expressam a ocorrência de múltiplos eventos e não têm relação com a concordância com os argumentos do verbo. Esses marcadores são normalmente afixos (frequentemente reduplicativos), mas podem também ser expressos por meio de supleção da raiz verbal. Em Karitiana, a pluracionalidade é expressa por meio da duplicação da raiz verbal e, em alguns casos, por supleção, como mostram os exemplos (150) e (151) abaixo, respectivamente (Storto 2012a, 2014):

(150) a. João Ø-naka-pon-Ø pikom kyynt.

João 3-DECL-atirar-NFUT macaco em

'O João atirou em macaco'

b. João $\varnothing$-na-pon-pon- $\varnothing \quad$ pikom kyynt.

João 3-DECL-atirar-DUPL-NFUT macaco em

'O João atirou em macaco (mais de uma vez)'

(151) a. João Ø-na-oky-t pikom.

João 3-DECL-matar.SG-NFUT macaco

'O João matou macaco' 
b. João Ø-na-popi-t pikom.

João 3-DECL-matar.PL-NFUT macaco

'O João matou macaco (mais de uma vez)'

Müller e Sanchez-Mendes (2008) defendem que os marcadores pluracionais em Karitiana realizam uma operação de pluralização na denotação cumulativa dos verbos, excluindo seus eventos atômicos (Ferreira 2005 para nomes e verbos e Müller 2000 para nomes). Essa operação é ilustrada pelo exemplo (152) que mostra que, quando o verbo possui um morfema pluracional, a sentença só pode ser utilizada em contextos em que João tenha caído mais de uma vez, não sendo apropriada para descrever um evento singular.

(152) João i-'ot-'ot-Ø.

João PART-cair-DUPL-ABS

'O João caiu (mais de uma vez)'

Situações: $\quad x$ uma vez

$\checkmark \quad$ mais de uma vez

Essa análise dos marcadores pluracionais é diferente da análise clássica da operação de plural descrita em Link (1983). O autor investigou a denotação de nomes contáveis e massivos utilizando uma estrutura algébrica de semirreticulado. Segundo sua proposta, nomes massivos denotam porções de matéria e nomes contáveis denotam átomos singulares cujos plurais são formados por operações sobre esses indivíduos singulares. Em Karitiana, a pluralização do domínio verbal é uma operação que exclui os átomos de uma denotação cumulativa (neutra para número) (Müller e Sanchez Mendes 2008). Não é o caso de que sentenças sem marcas de pluracionalidade denotem um evento singular. Sentenças sem nenhum tipo de quantificação adverbial como a pluracionalidade são compatíveis com denotações cumulativas, que incluem átomos e plurais. O exemplo (153), abaixo, mostra que o significado literal de uma sentença simples em Karitiana sem a presença de operadores de quantidade apresenta nomes e verbos com denotações de número neutro. 
Taso Ø-na-manga-t õwã.

homem 3-DECL-levantar-NFUT criança

'Um número indeterminado de homens levantou um número indeterminado de crianças um número indeterminado de vezes'

No entanto, isso não significa que as sentenças desse tipo em Karitiana são sempre ambíguas. A descrição literal é apenas uma tentativa de representar a indeterminação de número dos nomes e verbos na língua. Trata-se mais de um caso de vagueza ou indeterminação do que de ambiguidade.

Uma vez que a pluracionalidade é uma operação que subtrai os eventos atômicos de uma denotação cumulativa, uma sentença com afixo pluracional é adequada para descrever situações em que dois ou mais eventos ocorreram. As sentenças (154) e (155) com os advérbios sypom e myjym que correspondem a 'duas vezes' e 'três vezes', respectivamente, ilustram esse fato:

$$
\begin{aligned}
& \text { Sypom-t } \varnothing \text {-na-aka-t i-'ot-'ot- } \varnothing \quad \text { João. } \\
& \text { dois-OBL 3-DECL-COP-NFUT PART-cair-DUPL-ABS João } \\
& \text { 'O João caiu duas vezes' }
\end{aligned}
$$

\author{
(155) Õwã $\quad$ O-na-kot-kot-a-t mỹjym-t sypi. \\ criança 3-DECL-quebrar-DUPL-VERB-NFUT três-OBL ovo \\ 'Crianças quebraram ovo três vezes'
}

A análise dos afixos pluracionais em Karitiana como operadores de plural na denotação neutra para número do domínio verbal é diferente do que normalmente se observa em outras línguas que possuem pluracionalidade. Lasersohn (1995) investigou o fenômeno translinguisticamente e mostrou que os marcadores pluracionais estão usualmente associados a uma semântica de intensidade ou de um número de eventos acima da expectativa, como nos dados abaixo da língua Nahuatl (falada no México) e do Dyirbal (falado na Austrália). 
a. tlania Nahuatl

'perguntar'

b. $\quad$ tlatlania $^{61}$

'perguntar insistentemente'

(exemplos de Lasersohn 1995, p. 246)

(157)
a. balgan
Dyirbal
'golpear'
b. balbalgan
'golpear muito'

(exemplos de Lasersohn 1995, p. 246)

Os exemplos do Checheno (língua caucasiana) apresentados em Yu (2003) também apresentam a mesma característica. Os exemplos em (158) e (159) mostram que a pluracionalidade em Checheno está envolvida em uma repetição de muitos eventos e não exatamente em uma operação de plural ${ }^{62}$.
a. molu
Checheno
'beber'
b. myylu
'beber repetidamente'

(exemplos de Yu 2003, p. 293)

(159)
a. loocu
Checheno
'capturar'
b. loecu
'capturar repetidamente'

(exemplos de Yu 2003, p. 293)

${ }^{61}$ Em Nahuatl e em Dyirbal a pluracionalidade é expressa pela duplicação da primeira sílaba da raiz verbal.

62 Em Checheno, a pluracionalidade não é marcada por duplicação verbal, mas por meio de uma alternância da vogal temática. 
Como se pôde notar, a assunção de que os verbos têm denotação cumulativa em Karitiana, no sentido de ser neutra para número, ajuda a analisar apropriadamente a pluracionalidade na língua. Os marcadores pluracionais em Karitiana realizam uma operação de plural que exclui os átomos da denotação dos verbos. A próxima seção discute a propriedade de contabilidade associada aos predicados verbais em Karitiana.

\subsubsection{Contabilidade}

O objetivo desta seção é mostrar que a investigação de algumas propriedades dos quantificadores adverbiais em Karitiana apoia a hipótese de que todo o domínio verbal nessa língua é contável. Primeiramente, será apresentada a análise da distinção entre advérbios de frequência e de grau em francês (Doetjes 2007) para compará-los com os advérbios do Karitiana. Em seguida, algumas características da pluracionalidade em Karitiana serão retomadas para reforçar o argumento da contabilidade do domínio verbal.

\subsubsection{Advérbios de Frequência e de Grau em Francês}

Doetjes (2007) analisou a diferença entre as operações de frequência e de grau em francês analisando os advérbios souvent 'frequentemente/muitas vezes' e beaucoup 'muito'. De acordo com a autora, sentenças com advérbios de frequência, como souvent sempre possuem uma interpretação iterativa, ou seja, de ocorrência de muitos eventos. Essa é uma propriedade irrestrita desses advérbios que podem ocorrer tanto com predicados verbais télicos quanto atélicos, como mostram as sentenças (160) e (161), respectivamente.

(160) Sylvie va souvent au cinéma.

'A Sylvie vai frequentemente ao cinema'

(exemplo 1b de Doetjes 2007, p. 1)

(161) Il a plu souvent.

'Choveu frequentemente'

(exemplo 2a de Doetjes 2007, p. 2) 
Advérbios de grau como beaucoup, por outro lado, tem um comportamento diferente dependendo do tipo de predicado que modificam. Quando beaucoup é usado com predicados télicos, como no exemplo (162), a sentença tem um interpretação de muitos eventos no tempo. Sentenças com predicados atélicos como (163) têm leitura de intensidade, que Doetjes (2007) chama de leitura de grau.

(162) Sylvie va beaucoup au cinema.

'A Sylvie vai muitas vezes ao cinema'

(exemplo 1a de Doetjes 2007, p. 1)

(163) Il a plu beaucoup ${ }^{63}$.

'Choveu intensamente'

(exemplo 2a de Doetjes 2007, p. 2)

A análise de Doetjes (2007) para souvent e beaucoup é baseada na distinção contável-massivo dos predicados verbais. A autora assume a proposta de Bach (1986) de que predicados verbais télicos podem ser considerados como predicados contáveis e os atélicos como massivos. O autor sustenta que a mesma distinção empregada no domínio nominal entre predicados contáveis e massivos pode ser utilizada no tratamento dos predicados verbais. Bach (1986) mostra que há uma similaridade entre predicados atélicos e nomes massivos. Eles representariam a matéria (do inglês stuff) de que os predicados télicos e os nomes contáveis são constituídos. Assim, ouro, nome massivo, representa a matéria de que é feito o anel, nome contável. Do mesmo modo, correr, verbo atélico, seria a matéria de um predicado télico como correr até a farmácia.

Doetjes (2007) afirma que os advérbios de frequência como souvent podem contar em ambos os domínios, contável (télico) e massivo (atélico), porque eles possuem uma operação de plural de ocorrências (operação de $n$ vezes - do inglês $n$ times) que torna possível a multiplicação de eventos em qualquer contexto. A interpretação iterativa das sentenças com advérbios de frequência é, portanto, devida a uma propriedade do advérbio. Logo, ela sempre está disponível.

Já a interpretação iterativa de sentenças com advérbios de grau como beaucoup não

\footnotetext{
63 A sentença (163) parece ter também uma interpretação iterativa ('Choveu muitas vezes'). Doetjes (2007) assume que quando ela está disponível, o predicado atélico sofreu uma mudança de massivo para contável.
} 
pode ser explicada do mesmo modo, já que ela é restrita aos predicados télicos, como se viu em (162) e (163). De acordo com Doetjes (2007), advérbios de grau não possuem uma operação de plural como os advérbios de frequência. E uma vez que a interpretação de muitos eventos só está disponível em contextos télicos (contáveis), a autora afirma que ela é consequência da natureza contável do predicado, e não do advérbio. Quando os advérbios de grau ocorrem em sentenças com predicados atélicos (massivos) a sentença possui uma interpretação de grau. No entanto, Doetjes (2007) admite que uma leitura iterativa também é possível com alguns predicados atélicos modificados por beaucoup. Mas, uma vez que a autora assume que em sentenças com beaucoup a iteratividade é devida à natureza contável do predicado, ela tem que postular uma mudança do predicado de massivo para contável de predicados como pleuvoir 'chover' para capturar essa leitura em sentenças como (163). Desse modo, ela pode manter a assunção de que a interpretação de muitos eventos em sentenças com advérbios de grau tem origem na natureza contável do predicado, e não em uma característica especial do advérbio, como no caso dos advérbios de frequência.

\subsubsection{Advérbios de Frequência e de Grau em Karitiana}

O objetivo desta subseção é apresentar uma análise para advérbios de frequência e de grau em Karitiana. Essa análise será utilizada como argumento para a hipótese de que todo o domínio verbal da língua é contável. Advérbios de frequência em Karitiana sempre têm interpretação iterativa (muitos eventos), como o souvent do francês. Conforme discutido no capítulo 2, sentenças com o advérbio kanda(t) sempre denotam a ocorrência de muitos eventos, seja com predicados télicos, como 'ot 'cair' em (164), ou atélicos, como kydn 'esperar' em (165). A sentença em (165), por exemplo não pode usada em um contexto em que Inácio tenha esperado uma vez só por muito tempo.
(164)
João $\varnothing$-na-aka-t i-'ot-
João 3-DECL-COP-NFUT PART-cair-ABS muitas.vezes-ADV
'O João caiu muitas vezes' 
Inácio Ø-naka-kydn-Ø kanda-t.

Inácio 3-DECL-esperar-NFUT muitas.vezes-ADV

'O Inácio esperou muitas vezes'

Situações: $\quad \checkmark \quad$ 'O Inácio esperou muitas vezes'

× 'O Inácio esperou muito tempo em uma ocasião'

Logo, a operação de frequência em Karitiana parece ser semelhante à do francês. No entanto, algumas diferenças aparecem quando se compara o comportamento dos advérbios de grau nas duas línguas. A sentença (166) com o verbo de atividade pytim'adn 'trabalhar' e a (167) com o verbo estativo osedn 'estar alegre' ilustram que o advérbio de grau pita(t) pode ser usado para descrever uma situação iterativa ou com interpretação de grau ${ }^{64}$.

(166) João i-pytim'adn- $\varnothing$ pita-t ${ }^{65}$.

João PART-trabalhar-ABS pita-ADV

'O João trabalhou muito'

Situações: $\quad \checkmark \quad$ 'O João trabalhou muitas vezes'

$\checkmark$ 'O João trabalhou com muita intensidade'

(167) Inácio Ø-na-aka-t i-osedn- $\varnothing \quad$ pita-t.

Inácio 3-DECL-COP-NFUT PART-estar.alegre-ABS pita-ADV

'O Inácio ficou muito feliz'

Situações: $\quad \checkmark \quad$ 'O Inácio ficou feliz muitas vezes'

$\checkmark \quad$ 'O Inácio ficou feliz com muita intensidade'

O comportamento é semelhante ao de beaucoup em francês. No entanto, há duas propriedades cruciais que diferenciam pita(t) de beaucoup. Em primeiro lugar, diferentemente de beaucoup, que pode ocorrer como todos os tipos de predicados verbais, pita(t) só pode ser usado como modificador de grau, ou seja, com a noção de um grau acima do normal em uma escala, com predicados atélicos. Sentenças com pita(t) e predicados de accomplishments como 'a myhint gooj 'fazer uma canoa' em (168) ou achievements como otam 'chegar' em

\footnotetext{
64 Não vamos especificar o que seria propriamente essa leitura de grau porque ela será amplamente discutida no próximo capítulo. Por ora, ela está sendo associada aa uma leitura de intensidade.

${ }^{65}$ Pita(t) não está sendo traduzido nas glosas. O seu significado é parecido mas não exatamente igual ao muito do português. O próximo capítulo mostrará uma análise para o significado de pita(t).
} 
(169) têm uma interpretação não prevista para um modificador de grau. Esse comportamento de pita(t) com predicados télicos será discutido no próximo capítulo.

Yn Ø-naka-m-'a-t pita-t myhin-t gooj.

eu 3-DECL-CAUS-fazer-NFUT pita-ADV um-OBL canoa

'Eu fiz uma canoa mesmo'

(169) João i-otam-Ø pita-t.

João PART-chegar-ABS pita-ADV

'O João chegou mesmo'

Além disso, como será discutido em detalhes na próxima seção, pita(t) tem uma outra característica inesperada para advérbios de grau: ele pode ser usado em mais de uma situação associada ao que usualmente é chamado de contexto de grau, além do contexto iterativo. A sentença (170) pode ser usada em todos os contextos descritos em (171).
Inácio Ø-na-aka-t
i-pykyn-<a $>$-t pita-t.
Inácio 3-DECL-COP-NFUT PART-correr-ABS pita-ADV
'O Inácio correu muito'

(171) Situações em que a sentença (170) seria verdadeira:
a. 'O Inácio correu por muito tempo'
b. $\quad$ 'O Inácio correu muitas vezes'
c. 'O Inácio correu em alta velocidade'
d. 'O Inácio correu uma longa distância'
e. 'O Inácio correu fazendo muito esforço'

Portanto, pita(t) não pode ser analisado da mesma forma que beaucoup. Beaucoup possui um comportamento dicotômico nas suas interpretações; logo, faz sentido que ele seja explicado por uma regra binária, como a distinção contável-massivo. Pita(t), por outro lado, não possui um comportamento semelhante. Os dados (170) e (171) mostraram que o uso de pita(t) com predicados atélicos não está associado a um par de interpretações, mas a pelo 
menos três ${ }^{66}$. Não seria apropriado dizer que uma interpretação, a iterativa, pode ser explicada por uma regra de mudança de predicado e as outras interpretações por uma outra regra. Parece mais adequado apresentar uma proposta unificada para as interpretações. A tese que se pretende defender no próximo capítulo é a de que todas as interpretações são geradas por meio da modificação de grau. A interpretação iterativa é apenas mais uma interpretação presente nesse tipo de operação, tanto quanto as outras. A iteratividade será considerada uma dimensão de uma escala, e não uma operação no domínio verbal que compete com a modificação de grau. Esse mecanismo será esclarecido no capítulo 4.

Para analisar os advérbios de grau e de frequência em Karitiana assumimos que o domínio verbal não possui a mesma distinção contável-massivo encontrada no domínio nominal, mas é todo contável (Rothstein 1999, 2004, 2008). Para Rothstein (1999, 2004, 2008), a diferença entre predicados télicos e atélicos não revela uma diferença entre contável e massivo, como na proposta de Bach (1986), mas expressa de que modo o critério de atomicidade (MEAS), que cria os átomos semânticos para contagem, é preenchido.

Em predicados télicos como chegar, o que conta como uma unidade de evento é dado pelo léxico, como em (172a). A fórmula indica que o que conta como um evento de chegar é uma chegada. Por outro lado, predicados verbais atélicos como esperar não possuem átomos fornecidos pelo léxico, mas que são preenchidos contextualmente. Em outras palavras, a situação informa o que conta como um átomo de esperar, representado pela variável $\mathbf{U}$ na fórmula em (172b). Segundo a fórmula, o que conta como um evento de esperar é uma unidade $\mathbf{U}$ preenchida contextualmente.

$$
\begin{aligned}
& \text { a. }[[\text { chegar }]]=\lambda \text { e. } \operatorname{chegar}(\mathrm{e})^{\wedge} \operatorname{MEAS}(\mathrm{e})=<1, \text { chegada }> \\
& \text { b. }\left[[\text { esperar }]=\lambda \text { e. esperar }(\mathrm{e}){ }^{\wedge} \operatorname{MEAS}(\mathrm{e})=<1, \mathrm{U}>\right.
\end{aligned}
$$

Um dos argumentos de Rothstein (2008) para a assunção de que todo o domínio verbal é contável é que, aparentemente, não há classificadores nesse domínio como os encontrados no domínio nominal ${ }^{67}$. Os sintagmas verbais podem ser facilmente contados, diferentemente dos sintagmas adjetivais, por exemplo. As sentenças do inglês em (173)

\footnotetext{
${ }^{66}$ Como será defendido mais adiante, pelo menos três interpretações estão disponíveis nas sentenças com pita(t) como modificador de grau: duração no tempo, muitas ocorrências do evento e intensidade. Verbos de movimento apresentam ainda leitura de alta velocidade e longa distância.

${ }^{67}$ Paris (2011) e Donazzan (2013), por outro lado, defendem que, em chinês, há classificadores no domínio verbal.
} 
mostram que a operação de contagem só pode ocorrer nos adjetivos na presença do verbo to be 'ser/ficar'.

O advérbio iterativo three times 'três vezes' em (173a) só pode ser associado ao predicado principal made 'fez'. A sentença só possui a interpretação de que a bruxa fez por três vezes alguma coisa que deixou o João doente. Em (173b), por outro lado, o advérbio pode quantificar tanto o predicado principal (made 'fez') quanto o predicado da sentença subordinada (be 'ficar'). A sentença pode significar que a bruxa fez por três vezes alguma coisa que deixou o João doente ou que a bruxa fez algo (não sabemos quantas vezes) que deixou o João doente três vezes.

(173) a. The witch made John ill three times.

'A bruxa fez o João doente três vezes ${ }^{681}$

Situações: $\quad \checkmark$ fez alguma coisa três vezes

× ficar doente três vezes

b. The witch made John be ill three times.

'A bruxa fez o João ficar doente três vezes'

Situações: $\quad \checkmark$ fez alguma coisa três vezes

$\checkmark$ ficar doente três vezes

(exemplo 18 de Rothstein 2008, p. 12)

Os exemplos mostram que ill 'doente' não é um predicado que pode ser contado, enquanto que be ill 'ficar doente' pode. Isso mostra que o domínio das eventualidades como ill 'doente' são denotadas por predicados massivos, mas o domínio dos eventos como be ill 'ficar doente' é denotado por predicados verbais contávis. Essa é um distinção importante uma vez que a hipótese de Rothstein $(1999,2004,2008)$ não é a de que todos os predicados que denotam eventos sejam contáveis, mas a de que a contabilidade seja uma propriedade do domínio verbal. É possível haver eventualidades denotadas por predicados não verbais que são massivos, mas se há um sintagma verbal, ele é um sintagma contável.

Nas sentenças abaixo, uma vez que o predicado de (174a) não possui um verbo, a eventualidade não pode ser pluralizada e individualizada para ser distribuída aos participantes

68 Embora a sentença pareça agramatical em português, optamos por deixá-la o mais próximo possível da sentença em inglês para preservar o argumento de Rothstein (2008). 
John and Mary e a sentença é mal formada em inglês. Já (174b) é uma sentença bem formada porque o verbo be torna a pluralização da eventualidade possível, distribuindo-a aos participantes.

(174) a. \# The witch made John and Mary each ill.

'A bruxa fez John e Mary doentes individualmente'

b. The witch made John and Mary each be ill.

'A bruxa fez John e Mary ficar doentes individualmente'

(exemplo 20 Rothstein 2008, p. 12)

Em trabalhos anteriores, analisamos a distinção entre advérbios de frequência e de grau em Karitiana assumindo que todo o domínio verbal na língua é contável (SanchezMendes 2012a). A proposta foi a de que a interpretação de muitos eventos associada a advérbios de frequência como kanda(t) em Karitiana está sempre disponível porque o domínio verbal é todo contável na língua. Logo, a contagem de eventos é sempre uma possibilidade e não precisa ser precedida de uma operação times 'vezes' introduzida pelo advérbio, como na proposta de Doetjes (2007).

A operação de frequência com o advérbio kandat em Karitiana pode ser descrita como uma multiplicação das ocorrências dos eventos que pode ser aplicada em todos os contextos verbais na língua. A interpretação iterativa tem origem na natureza contável do predicado e não em uma propriedade particular do quantificador responsável pela contagem dos eventos, como sugerido por Doetjes (2007) para o souvent do francês. A tabela 34 abaixo resume as duas propostas apresentadas para os advérbios de frequência ${ }^{69}$.

Tabela 34: Comparação das Propostas para os Advérbios de Frequência

\begin{tabular}{|l|l|}
\hline \multicolumn{2}{|c|}{ Advérbios de Frequência } \\
\hline \multicolumn{1}{|c|}{ Doetjes (2007) } & \multicolumn{1}{c|}{ Nossa Proposta } \\
\hline $\begin{array}{l}\text { Iteratividade é devida a um } \\
\text { componente especial do advérbio. }\end{array}$ & $\begin{array}{l}\text { Iteratividade tem origem na natureza } \\
\text { contável do predicado. }\end{array}$ \\
\hline
\end{tabular}

\footnotetext{
${ }^{69}$ A nossa proposta é baseada na análise de Doetjes (2007) para a interpretação iterativa de sentenças com advérbios de grau.
} 
Uma vez que propusemos uma análise para a operação de frequência que possui uma operação a menos, já que não é preciso assumir que o advérbio possui um componente especial de times 'vezes', ela já parece mais econômica. Mas, assumir que o domínio verbal é contável é especialmente vantajoso na análise dos advérbios de grau. Como a sentença (170) mostrou, repetida em (175), pita(t) só pode ocorrer como modificador de grau em sentenças com predicados verbais atélicos e as sentenças apresentam mais de duas interpretações.

Inácio $\varnothing$-na-aka-t i-pykyn-<a>-t pita-t.
Inácio 3-DECL-COP-NFUT PART-correr-ABS pita-ADV
'O Inácio correu por muito tempo'
'O Inácio correu muitas vezes'
'O Inácio correu em alta velocidade'
'O Inácio correu uma longa distância'
'O Inácio correu fazendo muito esforço'

Uma vez que pita(t) ocorre como modificador de grau apenas com predicados atélicos e a interpretação de muitos eventos é tão importante quanto as outras, não parece adequado dizer que ela deve ser explicada por uma regra de exceção, como na proposta de Doetjes (2007). Na proposta da autora, o verbo tem que sofrer uma mudança de massivo para contável para ser pluralizado por beaucoup e a interpretação iterativa ficar disponível. Em uma proposta que assume que todo o domínio verbal é contável, a pluralização de eventos nesse contexto pode ser explicada do mesmo modo que com os advérbios de frequência, pela natureza contável do predicado.

Assumimos que a possibilidade de especificar um significado de grau (intensidade) é uma característica dos advérbios de grau que os advérbios de frequência não possuem. Logo, a proposta apresentada parece mais intuitiva, já que o advérbio que tem um componente gramatical a mais (de grau) é aquele cujas sentenças têm significados extras. O próximo capítulo tratará do modo como as diferentes interpretações das sentenças com pita(t) são geradas. Como já se pôde notar pelo exemplo (175) acima, pita(t) apresenta sempre um grau maior em relação a uma escala, ou seja, uma medida acima da média em uma escala, que pode variar (tempo, número de vezes, velocidade, etc.).

Portanto, uma proposta baseada em Rothstein $(1999,2004,2008)$ é mais vantajosa 
uma vez que a interpretação iterativa tem sempre a mesma origem, a multiplicação de eventos que está sempre disponível para predicados verbais. Na proposta de Doetjes (2007), por outro lado, a interpretação iterativa tem origens diferentes em sentenças com advérbios de frequência e de grau. A comparação das duas propostas é apresenta na tabela abaixo.

Tabela 35: Comparação das Propostas para os Advérbios de Grau

\begin{tabular}{|c|c|c|c|c|c|}
\hline \multicolumn{6}{|c|}{ Advérbios de Grau (AG) } \\
\hline \multicolumn{3}{|c|}{ Doetjes (2007) } & \multicolumn{3}{|c|}{ Nossa Proposta } \\
\hline $\begin{array}{l}\mathrm{AG}+ \\
\text { predicado } \\
\text { contável = } \\
\text { leitura } \\
\text { iterativa }\end{array}$ & \multicolumn{2}{|c|}{$\begin{array}{l}\text { Iteratividade com origem na } \\
\text { natureza contável do predicado }\end{array}$} & $\begin{array}{l}\mathrm{AG}+ \\
\text { predicado } \\
\text { télico }= \\
\text { leitura } \\
\text { iterativa }\end{array}$ & \multicolumn{2}{|c|}{$\begin{array}{l}\text { Iteratividade com origem na } \\
\text { natureza contável do predicado }\end{array}$} \\
\hline \multirow{2}{*}{$\begin{array}{l}\mathrm{AG}+ \\
\text { predicado } \\
\text { massivo }=\end{array}$} & $\begin{array}{l}\text { a) leitura } \\
\text { de grau }\end{array}$ & $\begin{array}{l}\text { Grau com origem } \\
\text { na natureza massiva } \\
\text { do predicado. }\end{array}$ & \multirow{2}{*}{$\begin{array}{l}\mathrm{AG}+ \\
\text { predicado } \\
\text { atélico }=\end{array}$} & $\begin{array}{l}\text { a) leitura } \\
\text { de } \\
\text { intensidade }\end{array}$ & $\begin{array}{l}\text { Com origem em } \\
\text { um componente } \\
\text { especial do } \\
\text { advérbio }^{71} \text {. }\end{array}$ \\
\hline & $\begin{array}{l}\text { b) } \\
\text { leitura } \\
\text { iterativa }\end{array}$ & $\begin{array}{l}\text { Mudança do verbo } \\
\text { de massivo para } \\
\text { contável. } \\
\text { Iteratividade com } \\
\text { origem na natureza } \\
\text { contável do } \\
\text { predicado. }\end{array}$ & & $\begin{array}{l}\text { b) leitura } \\
\text { iterativa }\end{array}$ & $\begin{array}{l}\text { Iteratividade com } \\
\text { origem na } \\
\text { natureza contável } \\
\text { do predicado }{ }^{72} \text {. }\end{array}$ \\
\hline
\end{tabular}

70 Essa é uma proposta para advérbios de grau como o beaucoup do francês. Em Karitiana, como se viu, predicados télicos não têm interpretação iterativa quando são modificados por pita(t), mas de uma confirmação do evento.

71 A natureza desse componente é o objeto de análise do capítulo 4.

72 A iteratividade será analisada no próximo capítulo como uma escala. A ideia é que por conta da natureza contável do domínio verbal, essa escala está sempre disponível. 


\subsubsection{Pluracionalidade}

Considerar o domínio verbal como contável ajudou a oferecer uma análise apropriada e econômica para advérbios de frequência e de grau em Karitiana. Nesta subseção, retomamos os dados de pluracionalidade na língua para reforçar o argumento. Como foi apresentado acima, os afixos pluracionais realizam uma operação de plural na denotação cumulativa (neutra para número) dos verbos em Karitiana. O plural é uma operação entendida semanticamente como um processo de individuação de um certo domínio. Uma vez que apenas os domínios que possuem unidades semânticas podem ser contados, a operação de plural pode ser usada como um teste para diagnosticar sintagmas contáveis (Chierchia 1998). Os exemplos em (176) do inglês demonstram esse teste. Footwear 'calçado' e blood 'sangue' são nomes massivos e não podem ser pluralizados, como mostram (176b) e (176d). Enquanto que shoe 'sapato' e drop 'gota' são contáveis e podem receber o morfema de plural (sentenças (176a) e (176c)).

(176) a. There are shoes in this store.

'Tem sapatos nesta loja'

b. * There are footwears in this store.

'Tem calçados nesta loja'

c. There are drops of blood on the wall.

'Tem gotas de sangue no muro'

d. * There are bloods on the wall.

'Tem sangues no muro'

(exemplo 1 de Chierchia 1998, p.55)

Vimos que a pluracionalidade é um mecanismo de plural no domínio verbal. Ela pode servir, então, como diagnóstico de sintagmas verbais contáveis. Em Karitiana, os afixos pluracionais podem ocorrer com qualquer tipo de predicado verbal e as sentenças sempre têm leitura iterativa. As sentenças (177) e (178) mostram que os predicados télicos de accomplishments como kokot Sete de Setembro dewota kyyn 'atravessar a rua Sete de Setembro' e de achievements como typ Antônio Bigode 'encontrar o Antônio Bigode' podem ser pluralizados, como previsto mesmo em uma proposta que assume a distinção contável- 
massivo no domínio verbal e propõe que esses sejam os predicados contáveis (Bach 1986 e Doetjes 2007).

a. João i-kokot- $\varnothing \quad$ Sete de Setembro dewota kyyn.

João PART-passar-ABS Sete de Setembro outro.lado para

'O João atravessou a rua Sete de Setembro'

b. João i-kokot-kokot- $\varnothing$ Sete de Setembro dewota kyyn.

João PART-passar-DUPL-ABS Sete de Setembro outro.lado para

'O João atravessou a rua Sete de Setembro (mais de uma vez)'

$(178)$

a. João Ø-naka-typ-Ø Antônio Bigode.

João 3-DECL-encontrar-NFUT Antônio Bigode

'O João encontrou o Antônio Bigode'

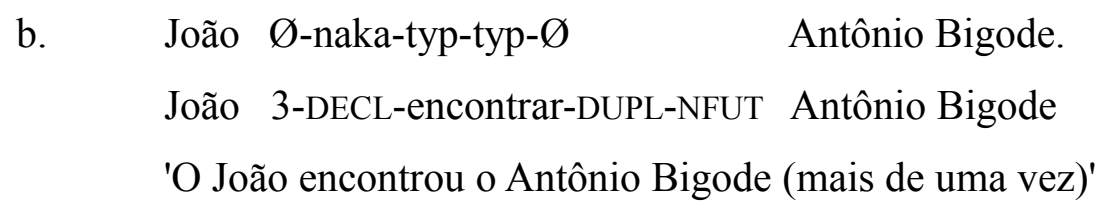

No entanto, predicados atélicos (atividades e estados stage-level) também podem ser pluralizados por duplicação verbal, como mostram os exemplos em (179) com o verbo de atividade pykyn 'correr' e (180) com o verbo estativo osedn 'estar alegre'. É importante notar que também nesses casos a interpretação da sentença é de iteratividade. Isso demonstra que a pluracionalidade está envolvida em uma pluralização de eventos também com esse tipo de predicado.

(179) a. João i-pykyn-<a>-t.

João PART-correr-ABS

'O João correu' 
b. João i-pykyn-pykyn-<a $>$-t.

João PART-correr-DUPL-ABS

'O João correu (mais de uma vez)'

Situações: $\quad \checkmark \quad$ correu mais de uma vez

× correu intensamente em uma ocasião

(180) a. Inácio Ø-na-aka-t i-osedn-Ø.

Inácio 3-DECL-COP-NFUT PART-estar.alegre-ABS

'O Inácio ficou alegre'

b. Inácio Ø-na-aka-t i-osedn-osedn- $\varnothing$.

Inácio 3-DECL-COP-NFUT PART-estar.alegre-DUPL-ABS

'O Inácio ficou alegre (mais de uma vez)'

Situações: $\quad \checkmark \quad$ ficou alegre mais de uma vez

× ficou muito alegre em uma ocasião

Em Karitiana, apenas predicados não eventivos, como os predicados estativos individual-level resistem a ser pluralizados, como mostram os exemplos (181) e (182) abaixo.

(181) a. Luciana i-kywytidn-<a>-t Botỹj-ty.

Luciana PART-acreditar-ABS Deus-OBL

'A Luciana acredita em Deus'

b. * Luciana i-kywytidn-kywytidn-<a>-t Botỹj-ty.

Luciana PART-acreditar-DUPL-ABS DEUS-OBL

Tradução pretendida: 'A Luciana acredita em Deus (mais de uma vez)'

(182) a. João Ø-na-aka-t Karitiana-t.

João 3-DECL-COP-NFUT Karitiana-ABS

'O João é Karitiana' 

b. * João Ø-na-aka-aka-t
Karitiana-t.
João 3-DECL-COP-DUPL-NFUT Karitiana-ABS
Tradução pretendida: 'O João é Karitiana' (mais de uma vez)'

Conforme a seção 1.2.4 mostrou, Kratzer (1995) propõe que predicados individuallevel não possuem argumento evento. Logo, vemos que a pluracionalidade em Karitiana se aplica a todos os predicados verbais que possuem um argumento evento, não se aplicando apenas a predicados verbais individual-level ${ }^{73}$.

Uma vez que todo o domínio verbal eventivo pode ser pluralizado em Karitiana, isso indica que esse domínio é inteiramente contável na língua. Segundo a proposta de Rothstein (1999, 2004, 2008), todo o domínio verbal é contável. A autora não diferencia predicados estativos eventivos (stage-level) e não eventivos (individual-level). Os dados do Karitiana motivam uma assunção um pouco mais restrita de que todo o domínio verbal eventivo é contável.

Segundo Rothstein $(1999,2004,2008)$, a diferença entre predicados télicos e atélicos não é uma distinção entre massivo e contável, mas uma diferença no modo como os átomos estão disponíveis para contagem. Os átomos que são contados em predicados télicos são dados pelo léxico, enquanto que os átomos dos predicados atélicos são dados pelo contexto. Nos dados do Karitiana mostrados, em (177) com kokot Sete de Setembro dewota kyyn 'atravessar a rua Sete de Setembro' e (178) com typ Antônio Bigode 'encontrar o Antônio Bigode', por exemplo, os átomos são cada evento de atravessar a Sete de Setembro e cada evento de encontrar o Antônio Bigode. Em (179) com o verbo pykyn 'correr' e (180) com o verbo osedn 'estar alegre', o contexto deverá fornecer o que conta como um evento de correr ou um evento de ficar alegre. Uma vez que os verbos kywytit Botyjty 'acreditar em Deus' e aka Karitiana 'ser Karitiana' nos dados (181) e (182) não possuem um domínio eventivo (contável), não é possível encontrar o que conta como um evento, mesmo considerando o contexto.

Logo, a tese adotada neste trabalho é a de que a individuação e a contagem não são necessariamente questões dependentes de átomo naturais, mas de átomos semânticos. A distinção contável-massivo e a operação de plural são fenômenos gramaticais que são relativamente independentes de operações cognitivas que exigem a percepção de indivíduos.

\footnotetext{
73 Um trabalho que ainda está por ser feito é examinar se as sentenças com pluracionalidade de predicados individual-level em Karitiana seriam possíveis se o predicado tivesse uma interpretação stage-level.
} 
A distinção contável-massivo é mais bem descrita em termos de como as expressões referem do que a que elas referem no mundo (Chierchia 1998, Rothstein 2010). Uma vez que é possível contar/pluralizar gramaticalmente os eventos denotados por todo tipo de predicado verbal eventivo em Karitiana, pode-se dizer que eles fazem parte de um domínio contável.

\subsection{Discussão Teórica}

Esta seção discute algumas consequências teóricas da afirmação de que o domínio verbal em Karitiana é cumulativo e contável. O objetivo é mostrar, primeiramente, que essas duas propriedades não são teoricamente excludentes. Em seguida, retomamos as questões debatidas na seção anterior deste capítulo e averiguamos quais as consequências da afirmação de que todo o domínio verbal é cumulativo para a distinção télico-atélico em Karitiana.

Dizer que um certo domínio tem denotação ao mesmo tempo cumulativa e contável pode gerar um certo desconforto devido ao fato de que a cumulatividade é uma propriedade tradicionalmente atribuída aos predicados massivos (Link 1983). No entanto, a cumulatividade não é a característica que distingue predicados massivos de contáveis. $\mathrm{Na}$ teoria de Link (1983), a cumulatividade é uma propriedade de predicados massivos e de plurais e, portanto, não é que o permite a distinção contável-massivo. Na proposta do autor, o teste para a distinção contável-massivo é baseado na propriedade da homogeneidade ${ }^{74}$.

À primeira vista, a cumulatividade e a homogeneidade parecem ser bastante similares. Mas, para descrever os predicados em trabalhos como este, a sua diferença é crucial. As definições em (183) e (184) (baseadas em Krifka 1992 e Rothstein 2010) mostram que a homogeneidade é uma propriedade dos predicados que é atribuída a suas partes, ou seja, é uma propriedade descendente. A cumulatividade, por outro lado, é uma propriedade ascendente, que caracteriza o predicado de acordo com as somas de suas partes.

\footnotetext{
74 Rothstein (2010), por outro lado, apresenta evidências de que nem a cumulatividade nem a homogeneidade podem distinguir seguramente predicados contáveis de massivos. Uma vez que a homogeneidade não é importante para este trabalho, vamos seguir a proposta clássica de Link (1983).
} 
(183) Homogeneidade:

Um predicado $\mathrm{P}$ é homogêneo se: para todo $\mathrm{x} \in \mathrm{P}$, e para todo $\mathrm{y}: \mathrm{y} \leq \mathrm{x} \wedge \neg \mathrm{y}=\mathrm{x}$ $\rightarrow \mathrm{y} \in \mathrm{P}$

Um predicado $\mathrm{P}$ é homogêneo se, e somente se, para todo $\mathrm{x}$ que pertence ao predicado $\mathrm{P}$, todas as partes $\mathrm{y}$ de $\mathrm{x}$, que forem diferentes de $\mathrm{x}$, também pertencem ao predicado $\mathrm{P}$.

(184) Cumulatividade:

Um predicado $\mathrm{P}$ é cumulativo se: $\mathrm{x} \in \mathrm{P} \wedge \mathrm{y} \in \mathrm{P} \wedge \neg \mathrm{x}=\mathrm{y} \rightarrow \mathrm{x} \square \mathrm{y} \in \mathrm{P}$

Um predicado $\mathrm{P}$ é cumulativo se, e somente se, dois indivíduos distintos x e y pertencem ao predicado $\mathrm{P}$, então, a soma de x e y também pertence a $\mathrm{P}$.

Como se vê, não há nada na definição de cumulatividade que impeça a operação de individuação e contagem de um predicado cumulativo. Na realidade, na própria definição de cumulatividade há uma operação de soma. De fato, a aproximação entre cumulatividade e predicados massivos se sustenta. Mas o fato é que todos os predicados massivos são cumulativos, mas nem todos os predicados cumulativos são massivos necessariamente. Rothstein (2010) mostrou, por exemplo, que há certos nomes contáveis em inglês como fence 'cerca' e line 'linha' que são cumulativos. Fence 'cerca' e line 'linha' podem ser pluralizadas (fences 'cercas' e lines 'linhas'); mas têm a propriedade da cumulatividade. Duas cercas (fences) que são colocadas lado a lado formam uma nova cerca. O mesmo vale para linha. Da mesma forma, predicados verbais atélicos, embora não possuam átomos em sua denotação, também são predicados contáveis.

Um outro aspecto teórico relacionado à afirmação de que os sintagmas verbais em Karitiana têm denotação cumulativa diz respeito à distinção télico-atélico. Conforme discutido na seção acima, Krifka (1998) assume que predicados quantizados são télicos e predicados cumulativos são atélicos. Nesse sentido, afirmar que todos os predicados verbais de uma língua são cumulativos seria o mesmo que dizer que eles são todos atélicos. A seção anterior já mostrou que não é esse o caso do Karitiana.

Rothstein (2008) mostra que há um certo equívoco em se assumir que todos os predicados cumulativos são atélicos. O equívoco se dá por conta da definição de cumulatividade. Para Krifka (1998), predicados atélicos como correr são cumulativos porque 
dois eventos adjacentes de correr formam um evento de correr. Predicados télicos como correr um quilômetro, por outro lado, não são cumulativos porque dois eventos adjacentes de correr um quilometro não formam um evento de correr um quilometro. Embora a operação de soma de dois eventos adjacentes pareça ser adequadamente descrito em termos da cumulatividade, Rothstein (2004) mostra que ela não é. Predicados cumulativos usuais se aplicam a átomos (ou pluralidades) e devolvem pluralidades. A soma de dois eventos atélicos adjacentes forma um outro átomo, um evento singular, e não uma pluralidade. Nesse ponto, os dois tipos de operação diferem.

A propriedade formal que captura adequadamente a propriedade de soma de dois eventos adjacentes formando um evento novo não é exatamente a cumulatividade. A essa propriedade formal, Rothstein (2004) dá o nome de s-cumulatividade. A sua definição é apresentada a seguir:

(185) S-cumulatividade:

$\exists \mathrm{e} \exists \mathrm{e}^{\prime}\left[\mathrm{X}(\mathrm{e}) \wedge \mathrm{X}\left(\mathrm{e}^{\prime}\right) \wedge \neg \mathrm{e} \subseteq \mathrm{e}^{\prime} \wedge \forall \mathrm{e} \forall \mathrm{e}^{\prime}\left[\mathrm{X}(\mathrm{e}) \wedge \mathrm{X}\left(\mathrm{e}^{\prime}\right) \wedge \mathrm{R}\left(\mathrm{e}, \mathrm{e}^{\prime}\right) \rightarrow \mathrm{X}\left({ }^{\mathrm{S}}\left(\mathrm{e} \cup \mathrm{e}^{\prime}\right)\right)\right]\right]$

Um predicado $\mathrm{X}$ é s-cumulativo se, para qualquer duas instâncias do predicado $\mathrm{X}$ relacionadas por uma relação $\mathrm{R}$, elas podem ser somadas formando uma entidade singular que também é uma instância do predicado X.

A relaçãao $\mathbf{R}$ refere-se à adjacência temporal, ou seja, os dois eventos e e e' podem ser somados e o resultado da soma é uma entidade singular. A propriedade da scumulatividade separa os predicados de estado e de atividade que são s-cumulativos dos predicados de accomplishment e achievement que não são s-cumulativos. Dois eventos adjacentes de amar ou de correr podem ser conceptualizados como um único evento que é também, por sua vez, um evento de amar e de correr. Dois eventos adjacentes de comer três maçãs ou de alcançar o topo da montanha não podem ser somados para formar um único evento de comer três maçãs ou alcançar o topo da montanha. Nesse sentido, quando Krifka (1992, 1998) assume que os predicados cumulativos são atélicos, ele está empregando na verdade a propriedade da s-cumulatividade.

Uma vez esclarecida a diferença entre cumulatividade e s-cumulatividade, o problema se dezfaz. Não há mais o comprometimento de se assumir que todos os VPs em Karitiana são atélicos com a afirmação de que eles são cumulativos. A denotação dos 
sintagmas verbais é cumulativa no sentido que ela possui átomos e somas. Em em sua denotação há tanto predicados télicos quanto atélicos. Os predicados télicos como comer três maças e alcançar o topo da montanha também são cumulativos. A propriedade que eles não compartilham com os predicados atélicos como correr e amar é a s-cumulatividade.

\subsection{Considerações Finais}

Esta seção mostrou argumentos de que o domínio verbal eventivo em Karitiana tem denotação ao mesmo tempo cumulativa (neutra para número) e contável. O comportamento da pluracionalidade na língua apoia a hipótese da cumulatividade e o comportamento dos quantificadores adverbiais e da pluracionalidade apoiam a hipótese da contabilidade dos predicados verbais eventivos. Além disso, discutiu-se brevemente as consequências teóricas da hipótese. Primeiramente, foi mostrado que e a teoria semântica sustenta essas duas propriedades, a despeito de sua aparente incompatibilidade. Em seguida, as propriedades de cumulatividade e s-cumulatividade foram discutidas a fim de resolver um problema acerca da distinção télico-atélico. A conclusão geral da seção é de que os sintagmas verbais em Karitiana são: (i) cumulativos (no sentido de neutros para número), ou seja possuem átomos e somas em sua denotação; (ii) contáveis, porque possuem átomos semânticos disponíveis para contagem; e (iii) divididos em télicos ou atélicos, e todos eles têm denotação cumulativa e contável. 


\section{Capítulo 4}

\section{A Modificação de Grau}

\section{Introdução}

O objetivo deste capítulo é apresentar uma análise para a modificação de grau exercida pelo advérbio pita(t) em Karitiana. O capítulo está dividido em cinco partes. A primeira seção discutirá de forma abrangente algumas características dos modificadores de grau mostrando de que forma sua análise é relevante para a teoria da Semântica Formal. Na seção 2, a teoria da Semântica Escalar é apresentada. A seção 3 apresenta os dados da modificação de grau em Karitiana. A seção 4 apresenta uma proposta de formalização unificada para pita(t) em todos os domínios em que ocorre. A seção 5 discute, por fim, algumas consequências da tese defendida no capítulo.

\section{Grau: uma Noção Especial}

O foco desta seção é discutir algumas características peculiares dos chamados modificadores de grau. O objetivo é mostrar de que modo esses modificadores representam um desafio para a análise semântica.

A primeira propriedade dos modificadores de grau que os particulariza é o fato de que possuem uma certa liberdade em relação ao tipo de constituintes que modificam que não é compartilhada por nenhum outro tipo de advérbio. A distribuição da palavra muito em português apresentada em (186) ilustra essa liberdade:

(186) a. Uma pessoa [ muito inteligente ] resolve as coisas rapidamente. ADJETIVO

b. O João [ correu muito ].

VERBO

c. O João correu [ muito rápido ].

ADVÉRBIO

As possibilidades acima são apenas aquelas em que muito é um advérbio. Ele tem, ainda, um uso como quantificador nominal em português, como mostram as sentenças em (187). 
(187) a. [ Muito homem ] gosta de futebol.

NOME CONTÁVEL SINGULAR

b. [ Muitos homens ] gostam de futebol.

NOME CONTÁVEL PLURAL

c. [ Muito ouro ] foi roubado do banco.

NOME MASSIVO

A possibilidade de advérbios desse tipo modificarem ou quantificarem vários domínios interfere na própria definição de toda a classe dos advérbios em português. Nas gramáticas tradicionais, é comum se encontrar como definição de advérbio a classe de palavras que modifica adjetivos, verbos e outros advérbios. No entanto, apenas os advérbios de grau (que são tratados como de intensidade nessas gramáticas) possuem essa característica. As outras classes de advérbios se aplicam regularmente aos sintagmas verbais ${ }^{75}$.

Uma outra propriedade dos modificadores de grau é o número de palavras disponíveis para expressar essa operação nas línguas. Algumas línguas possuem palavas diferentes para muito dependendo do domínio que está sendo modificado. Os exemplos (188) e (189) abaixo mostram respectivamente dados do inglês e do francês que ilustram essa diferença. Very do inglês e très e trop do francês modificam adjetivos e advérbios, enquanto que a lot do inglês e beaucoup do francês modificam predicados verbais ${ }^{76}$.

a. John is [ very clever ].

ADJETIVO

'O John é muito inteligente'

b. John [ ran a lot ].

VERBO

'O John correu muito'

c. John ran [ very fast ].

ADVÉRBIO

'O John correu muito rápido'

(189) a. Jean est [ très / trop intelligent ].

ADJETIVO

'O Jean é muito inteligente'

b. Jean [ a beaucoup couru ].

VERBO

'O Jean correu muito'

\footnotetext{
75 Não estamos considerando aqui os advérbios sentenciais como infelizmente, ou os orientados para o sujeito como inteligentemente. A ideia é apenas refletir um pouco sobre a definição das gramáticas tradicionais. De todo modo, esses advérbios não modificam adjetivos e advérbios.

76 Há ainda a diferença em inglês entre many e much 'muito' como determinantes de nomes contáveis e massivos, respectivamente.
} 
c. Jean a couru [ très / trop vite ].

ADVÉRBIO

' O Jean correu muito rápido'

Além dessa diferença vocabular em relação ao domínio modificado, as palavras que expressam grau elevado possuem uma quantidade robusta de quase-sinônimos formais ou informais (Guimarães 2007). Em (190a) e (190b), por exemplo, encontram-se quasesinônimos formais de muito em português. Em (190c), apresenta-se uma pequena seleção de seus quase-sinônimos informais ${ }^{77}$.

(190) a. sobremaneira; assaz; demasiado; bastante;

b. completamente; excessivamente; profundamente; demasiadamente;

c. pra burro; pra chuchu; pra dedéu; pacas; super.

Além dessas propriedades especiais dos modificadores de grau, uma característica formal que eles possuem e que tornam desafiadora a sua análise é o fato de eles não poderem ser tratados do mesmo modo que os outros modificadores verbais como os de tempo e de modo. O primeiro capítulo desta tese mostrou que os modificadores fazem uma espécie de restrição no domínio ao qual se aplicam e que essa restrição é expressa na forma lógica por uma conjunção. A forma lógica em (191b) pode ser lida da seguinte forma: existe um evento de correr, João é o agente desse evento e esse é um evento rápido.

(191) a. $\quad$ O João correu rápido.
b. $\quad \exists$ e [ correr (e) \& Agente (João) (e) \& rápido (e) ]

Essa não é, no entanto, uma análise apropriada para os modificadores de grau porque eles não fazem uma restrição no domínio verbal do mesmo modo que um advérbio como rápido. A forma lógica (191b) não descreve apropriadamente o significado da sentença (191a). Não é adequado descrever a sentença como: existe um evento de correr, João é o agente desse evento e esse evento é muito.

Estamos utilizando a terminologia quase-sinônimos porque não queremos nos comprometer com a análise de todos esses itens. Muitos deles parecem ter um significado diferente de muito. 
a. O João correu muito.

b. $\quad \exists e[$ correr (e) \& Agente (João) (e) \& muito (e) ]

Essa característica dos modificadores de grau tornam sua pesquisa, do ponto de vista semântico, bastante desafiadora. É preciso investigar de que modo eles entram na composição semântica e como sua interpretação pode ser capturada pela forma lógica. O objetivo das próximas seções será o de propor uma análise apropriada para esse tipo de advérbio, investigando especificamente o advérbio pita(t) do Karitiana.

\section{Semântica Escalar}

O objetivo desta seção é apresentar de que modo a Semântica Formal tem tratado as construções que envolvem grau. O estudo linguístico dessa noção esteve tradicionalmente vinculado à investigação dos adjetivos e das estruturas de comparação, mas esse instrumental vem sendo aplicado com sucesso na análise de outras construções, como a modificação de grau em diferentes domínios. A teoria que será apresentada a seguir é normalmente chamada de Semântica Escalar e segue textos clássicos sobre o tópico e que são usados nos estudos sobre o assunto: Kennedy (1999), Kennedy e McNally (2005) e Kennedy (2007).

A modificação de grau é entendida, de modo geral, como uma operação sobre predicados que são graduáveis. Uma propriedade tradicionalmente atribuída a predicados graduáveis é a sua possibilidade de ocorrência em estruturas comparativas. As sentenças abaixo mostram quatro adjetivos graduáveis, alto, caro, aberto e cheio, em sentenças comparativas.

(193) a. O João é mais alto do que o Pedro.

b. O café importado é mais caro do que o nacional.

c. A porta da direita está mais aberta do que a da esquerda.

d. O meu copo está mais cheio do que o seu.

Predicados não graduáveis, por outro lado, resistem a aparecer em estruturas comparativas: 
(194) a. ?O João é mais vegetariano do que o Pedro.

b. ? A Maria está mais grávida do que a $\mathrm{Ana}^{78}$.

c. ? O número 7 é mais primo do que o 3 .

d. ? Esta figura é mais hexagonal do que aquela ${ }^{79}$.

Um outro diagnóstico clássico para distinguir predicados graduáveis de não graduáveis é o uso de modificadores de grau como muito e tão. Somente predicados graduáveis podem aparecer com esses modificadores:

(195) a. O João é muito/tão alto.

b. $\quad$ O café está muito/tão caro.

c. A porta está muito/tão aberta.

d. O copo está muito/tão cheio.

e. ? O João é muito/tão vegetariano.

f. ? A Maria está muito/tão grávida.

g. ? O número 7 é muito/tão primo.

h. ? Esta figura é muito/tão hexagonal.

Kennedy e McNally (2005) propõem uma tipologia semântica para os predicados graduáveis segundo duas propriedades principais:

(i) divisão em relativos e absolutos em relação a um parâmetro de comparação;

(ii) relação a uma estrutura escalar aberta ou fechada.

78 Os exemplos (194a) e (194b) são possíveis se forem acomodados e interpretados, por exemplo, como 'O João é vegetariano há mais tempo do que o Pedro (ou é mais radical do que ele)' e 'A Maria está grávida há mais tempo do que a Ana'. No entanto, nem com acomodação é possível comparar a propriedade de ser primo ou a propriedade de ser hexagonal

79 Estou seguindo os estudos tradicionais em Semântica Escalar que colocam um ponto de interrrogação nessas sentenças e não um asterisco de agramaticalidade. 


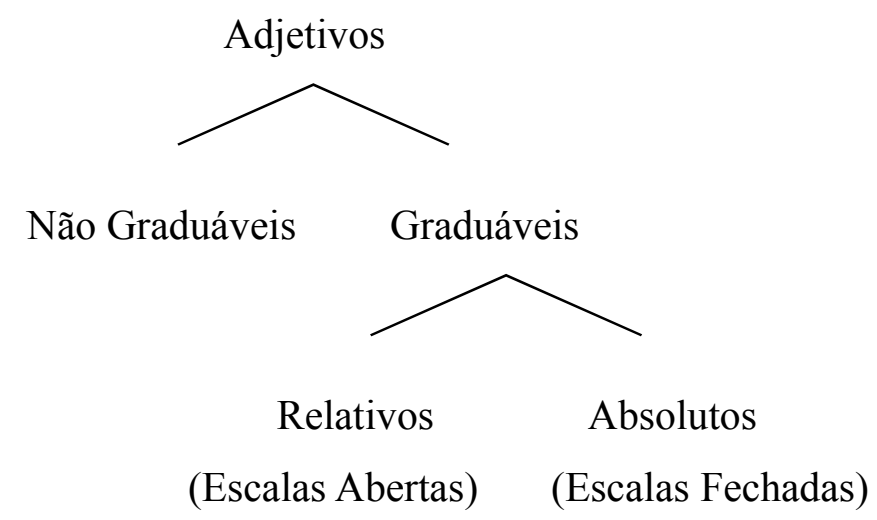

Cada uma dessas propriedades será apresentada em detalhes nas subseções que seguem.

\subsection{Predicados Relativos vs Predicados Absolutos}

O objetivo desta subseção é apresentar a divisão dos predicados graduáveis em relativos e absolutos segundo a Semântica Escalar (Kennedy 1999, Kennedy e McNally 2005 e Kennedy 2007). A proposta dessa divisão é separar os predicados de acordo com a sua relação com o contexto. Os predicados relativos são aqueles que dependem de um parâmetro de comparação contextual (do inglês standard of comparison) para serem interpretados, enquanto que os absolutos não possuem um parâmetro de comparação dependente do contexto. Por exemplo, o adjetivo alto é um predicado relativo porque sua denotação varia contextualmente. Para se atribuir a uma entidade a propriedade de ser alto é preciso se levar em conta em relação a que está sendo designada essa qualidade. O parâmetro de comparação é determinado de acordo com uma classe de comparação. A classe de comparação é formada por um conjunto de entidades que são similares em algum sentido com o que indivíduo em questão. Esse conjunto é selecionado contextualmente e influencia na denotação do predicado. A classe de comparação pode ser explicitada por um sintagma preposicional do tipo 'para uma criança' ou 'para um jogador de basquete'. Predicados relativos são, portanto, aqueles cujas condições de verdade variam de acordo com uma classe de comparação. A sentença abaixo pode ser verdadeira se for avaliada segundo um parâmetro de comparação criado a partir da classe de crianças; mas pode ser falsa se a classe de comparação considerada for de jogadores de basquete. 
Já um adjetivo como cheio não tem seu parâmetro de comparação definido contextualmente. Não é necessário que se compare contextualmente o que é cheio para se afirmar que algo está cheio. Se um copo for considerado cheio, não é possível encontrar um contexto que faça, em comparação com outros copos, ele ser considerado um copo vazio. Uma vez que esses adjetivos são independentes de um parâmetro de comparação, é estranho utilizar esse tipo de predicado com uma classe de comparação explícita, como mostram os exemplos abaixo:
a. ? Meu copo está cheio para um copo.
b. ? A porta da direita está aberta para uma porta.

Kennedy e McNally (2005) chamam a atenção para casos em que os adjetivos absolutos podem ser usados de modo impreciso. Em uma sentença como (198), por exemplo, a afirmação não é a de que o restaurante está completamente vazio, sem cadeiras, mesas ou mesmo alguns clientes. O uso de vazio é relativizado segundo um parâmetro de comparação que leve em conta outros restaurantes ${ }^{80}$.

(198) O restaurante está vazio.

A tabela abaixo resume essas características:

\footnotetext{
${ }^{80}$ Uma análise alternativa para o uso impreciso de adjetivos absolutos é por meio do que Lasersohn (1999) chama de halo pragmático, que utiliza a noção de aproximação da verdade. $\mathrm{O}$ halo pragmático considera situações próximas da verdade como verdadeiras com um propósito prático. Nesse caso, um restaurante quase vazio pode ser considerado vazio por uma questão pragmática. Ver Lasersohn (1999) para mais detalhes.
} 
Tabela 36: Predicados Graduáveis e sua Relação com o Parâmetro de Comparação

\begin{tabular}{|l|c|c|}
\hline \multicolumn{1}{|c|}{ Tipos de Predicado } & $\begin{array}{c}\text { Relação com o Parâmetro de } \\
\text { Comparação Contextual }\end{array}$ & Exemplos \\
\hline Relativo & Dependente & $\begin{array}{c}\text { alto, baixo, } \\
\text { caro, barato }\end{array}$ \\
\hline Absoluto & Independente & $\begin{array}{c}\text { aberto, fechado } \\
\text { cheio, vazio }\end{array}$ \\
\hline
\end{tabular}

Uma outra característica que diferencia adjetivos relativos e absoutos é a sua relação com casos limítrofes (do inglês borderline cases) e com o paradoxo sorites (Kennedy 2007). Apenas adjetivos relativos estão associados a casos limítrofes e ao paradoxo sorites. Casos limítrofes são aqueles em que é difícil fazer uma avaliação da sentença. A sentença abaixo, por exemplo, é claramente verdadeira em alguns casos (se a classe de comparação for os cafés das cidades italianas) e claramente falsa em outros (se a classe de comparação for os cafés de cidades com alto custo de vida). No entanto, há casos em que não é tão fácil fazer esse julgamento (se a classe de comparação for o conjunto das capitais européias com economia semelhante à de Roma). Esse casos são chamados de casos limítrofes.

(199) O café em Roma é caro.

(baseado no exemplo 1 de Kennedy 2007, p.2)

O paradoxo sorites, por sua vez, é um paradoxo baseado em uma estrutura de argumentos que parece válida, mas que leva a uma conclusão falsa. O exemplo em (200) ilustra essa estrutura.

(200) P1: Uma xícara de café de 10 reais é cara (para uma xícara de café).

P2: Qualquer xícara de café que custe 1 centavo a menos que uma xícara de café cara é uma xícara de café cara.

C: $\quad$ Então, qualquer xícara de café de graça é uma xícara de café cara. 
O problema desse paradoxo está na segunda premissa, que quando aplicada sucessivamente leva a uma conclusão falsa. Essa premissa é falsa, embora não seja facilmente reconhecida como falsa. Segundo Kennedy (2007), apenas os predicados relativos estão associados a casos limítrofes e ao paradoxo sorites porque eles são vagos (por oposição aos absolutos que não são vagos). Segundo o autor, os predicados vagos são esses em que é difícil julgar dois indivíduos que são associados a graus muito semelhantes. Um café de R\$10,00 e um de R\$ 9,99 são ambos caros (em um certo contexto). Mas é difícil avaliar em que momento a diferença de 1 centavo poderia determinar se um café é caro ou barato. Segundo Kennedy (2007), por um princípio metalinguístico, os falantes preferem não se comprometer com a posição de julgar dois graus muito próximos (como a diferença de apenas 1 centavo, por exemplo, como iguais ou diferentes, daí a dificuldade de avaliação. A próxima subseção vai apresentar uma outra característica que separa os predicados relativos e absolutos, a sua estrutura escalar.

\subsection{Estrutura Escalar}

O objetivo desta subseção é demonstrar por que se assume que os predicados graduáveis estão associados a uma escala (Kennedy 1999, Kennedy e McNally 2005). A Semântica Escalar assume uma relação biunívoca entre graus e escalas. Kennedy (1999) afirma que a abordagem escalar para a investigação do grau é empiricamente superior à análise que não faz uso de escalas como a análise dos adjetivos como predicados vagos ${ }^{81}$. Vamos seguir a proposta de Kennedy (1999) e assumir que predicados graduáveis estão associados a estruturas escalares.

No modelo da Semântica Escalar, escalas são construtos formais definidos como um conjunto de graus ordenados em uma dimensão. Elas podem ser consideradas metaforicamente uma reta formada por graus ordenados em uma determinada dimensão (que pode ser peso, altura, temperatura, comprimento, etc). Cada dimensão está relacionada a uma escala diferente, ou seja, a dimensão velocidade está associada a uma escala de velocidade, a dimensão altura a uma escala de altura e assim por diante. Toda entidade pode ser associada a um grau de diversos tipos de escala. Por exemplo, na escala de altura, o indivíduo Luciana, que tem 1,61 m de altura, está associado a um grau abaixo do grau associado ao indivíduo

${ }_{81}$ Para um estudo da análise dos adjetivos graduáveis como predicados vagos ver Klein, E. (1980). 
Oscar, que mede $2,05 \mathrm{~m}^{82}$.

(201)

$\begin{array}{ll}1,61 \mathrm{~m} & 2,05 \mathrm{~m} \\ \text { Luciana } & \text { Oscar }\end{array}$

Com o uso formal das escalas, é possível explicar estruturas linguísticas que expressam comparação em relação a alguma dimensão. Uma vez que cada indivíduo está associado a um grau de uma escala, ele pode ser comparado aos demais em relação a essa escala. Por exemplo, a sentença O Oscar é mais alto do que a Luciana será verdadeira se, no contexto em que for usada, for possível estabelecer formalmente uma escala de altura em que o indivíduo Oscar esteja associado a um grau que seja superior ao grau associado ao indivíduo Luciana. Um contexto associado a uma escala como em (201) acima seria um contexto em que a sentença é verdadeira.

Além disso, é possível marcar um grau na escala que corresponda ao que é considerado como o grau normal para aquela dimensão e contexto, possibilitando a interpretação de modificações de grau como muito e pouco, que são construídas em comparação a esse normal. Assim, por exemplo, um indivíduo é muito alto se está associado a um grau bem acima de um $\mathbf{n}$ que é assumido como normal de altura na escala construída para aquele contexto. Suponha que a altura normal para um ser humano adulto do sexo masculino no sul do Brasil seja algo em torno de 1,75 m. Como Oscar mede 2,05 m, ele pode ser considerado muito alto nesse contexto. É importante lembrar que o valor desse $\mathbf{n}$ normal varia segundo o contexto. Em um contexto em que Luciana tem 11 anos e está sendo comparada com as outras crianças dessa idade, medindo 1,61 m, ela será muito alta. Mas se o parâmetro de comparação for de mulheres adultas jogadoras de basquete, ela será bastante baixa.

Segundo Kennedy e McNally (2005), as escalas associadas aos predicados graduáveis são divididas em dois tipos: abertas e fechadas. Escalas abertas são aquelas que não possuem um grau mínimo ou máximo lexicalmente determinados. Alto, por exemplo, é um adjetivo de escala aberta, uma vez que não há lexicalmente definido um grau mínimo para algo ser considerado alto. Do mesmo modo, não há um grau máximo determinado para altura. Não existe lexicalmente representado um ponto máximo de altura a que uma entidade pode

\footnotetext{
82 A palavra indivíduo aqui está sendo tomada do ponto de vista da Semântica Formal, em oposição ao conceito de predicado. Indivíduos não são, necessariamente, pessoas.
} 
chegar. A figura abaixo representa esse tipo escala. As extremidades sem preenchimento representam os polos abertos da escala.

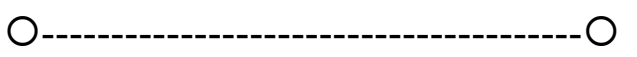

ESCALA ABERTA

Por outro lado, as escalas fechadas possuem um grau mínimo ou um grau máximo determinados. Por exemplo, cheio e vazio são adjetivos de escala fechada. A escala ocupação associada a esses adjetivos possui um grau mínimo, associado a vazio, e um grau máximo, associado a cheio. Há ainda adjetivos de escala fechada que têm apenas um dos polos fechados. Por exemplo, molhado/seco são fechados apenas no grau mínimo representado pelo seco. Seco é o polo fechado da escala porque um indivíduo deve estar completamente seco para receber esse adjetivo. Já molhado representa o polo aberto porque basta um pouco de líquido para se considerar um indivíduo como molhado e não há um limite definido lexicalmente como grau máximo de molhado ${ }^{83}$. Por outro lado, perigoso/seguro são fechados apenas no grau máximo representado por seguro. Qualquer grau abaixo de seguro já representa algo perigoso na escala de segurança. As representações abaixo revelam as três possibilidades de escalas fechadas ${ }^{84}$.

a.

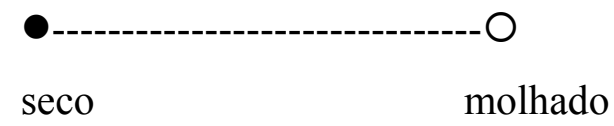

b.

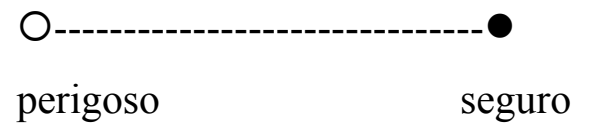

c.

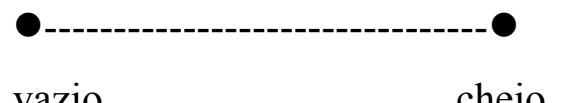

ESCALA PARCIALMENTE FECHADA

FECHADA NO GRAU MÍNIMO

ESCALA PARCIALMENTE FECHADA

FECHADA NO GRAU MÁXIMO

ESCALA TOTALMENTE FECHADA

83 Um tipo de grau máximo de molhado pode ser atingido se toda a extensão de uma roupa, por exemplo, estiver molhada. Mas nesse caso, o grau máximo é dado pelo indívíduo e não pela propriedade. Uma roupa completamente molhada é uma roupa que está (minimamente) molhada em todas as suas partes e não uma roupa que atingiu o grau máximo de propriedade de ser molhado.

${ }^{84}$ A princípio, parece não haver diferença entre ser fechado no grau mínimo ou no grau máximo. Se os adjetivos perigoso/seguro forem considerados como associados a uma escala de periculosidade, a escala pode ser considerada como fechada no grau mínimo. Frutos (2011) defende que os predicados de escalas parcialmente fechadas podem ser tratados de modo uniforme. 
Somente os adjetivos de escala fechada podem aparecer em sentenças com modificadores proporcionais como os advérbios completamente em (204a) e parcialmente, em (204b).

(204) a. O copo está completamente cheio/vazio.

b. O copo está parcialmente cheio/vazio.

Uma vez que não há um ponto em que se pode fechar a escala, os adjetivos de escala aberta não podem ser usados com completamente e parcialmente, como se vê em (205).

(205) ? O jogador é completamente/parcialmente alto.

A estrutura da escala influencia na escolha do parâmetro de comparação contextual, descrita na subseção anterior. Adjetivos associados a escalas totalmente abertas (como alto) estão relacionados a parâmetros de comparação relativos, ou seja, dependentes do contexto; enquanto que adjetivos graduáveis que usam escalas fechadas (como cheio) relacionam-se a parâmetros absolutos, ou seja, independentes do contexto. As relações dessas características estão sintetizadas na tabela abaixo:

Tabela 37: Estrutura Escalar e o Parâmetro de Comparação

\begin{tabular}{|c|c|c|}
\hline $\begin{array}{c}\text { Natureza do Predicado quanto } \\
\text { ao Parâmetro de Comparação }\end{array}$ & $\begin{array}{c}\text { Natureza do Predicado } \\
\text { quanto à Estrutura Escalar }\end{array}$ & Exemplos \\
\hline Relativo & Predicado de Escala Aberta & $\begin{array}{c}\text { alto, baixo, } \\
\text { caro, barato }\end{array}$ \\
\hline Absoluto & Predicado de Escala Fechada & $\begin{array}{c}\text { aberto, fechado, } \\
\text { cheio, vazio }\end{array}$ \\
\hline
\end{tabular}

Uma vez descritas a relação com o padrão de comparação e a estrutura escalar dos predicados graduáveis, vamos explorar qual a relevância dessas distinções para a teoria linguística. 


\subsection{Relevância do Parâmetro de Comparação e da Estrutura Escalar}

Esta seção mostra que a divisão apresentada acima é uma classificação teórica de diferentes tipos de predicados que tem respaldo descritivo. Pretende-se mostrar que a distinção baseada no parâmetro de comparação e na estrutura escalar é relevante para a análise da distribuição e do significado de modificadores (Kennedy e McNally 2005).

Kennedy e McNally (2005) investigaram a distribuição dos modificadores do inglês very, much 'muito' e well 'bem' com adjetivos deverbais (particípios). A observação dos autores é de que esses advérbios apresentam uma distribuição complementar que não é possível de ser explicada apenas do ponto de vista sintático. Os dados em (206) abaixo mostram que esses modificadores selecionam diferentes tipos de adjetivos:

(206) a. Al was very (?well/?much) surprised by the results of the election.

'O Al estava muito surpreso com o resultado das eleições'

b. Their vacation was much (?well/?very) needed.

'As suas férias eram muito necessárias'

c. Martin Beck was well (?much/?very) acquainted with the facts of the case.

'O Martin Beck estava bem familiarizado com os fatos do caso'

(exemplo 1 de Kennedy e McNally 2005, p.345)

Segundo Kennedy e McNally (2005), a distribuição desses três advérbios está relacionada à tipologia das escalas associadas aos adjetivos, assim como à relação desses adjetivos com o parâmetro de comparação. O advérbio very, por exemplo, só pode ser usado com adjetivos relativos, como surprised 'surpreso', em (206a). Surprised é um predicado relativo porque possui um parâmetro de comparação dependente do contexto: quando se diz que Al estava muito surpreso, leva-se em conta outras possíveis situações de Al ficar surpreso. O possível uso de very com adjetivos absolutos está associado a uma relativização do adjetivo. Por exemplo, em (207) abaixo, o uso do adjetivo empty 'vazio' foi relativizado. Conforme discutido anteriormente, não se trata de vazio em seu sentido absoluto, sem garçons, clientes, ou móveis, mas de um uso impreciso que, na realidade, quer dizer, quase 
vazio. Os autores não avançam muito na análise desse uso, mas o utilizam apenas para mostrar que a propriedade de very selecionar apenas predicados relativos é tão forte que seu uso com predicados absolutos os relativiza.

(207) The restaurant is very empty tonight.

'O restaurante está muito vazio esta noite'

(exemplo 70 de Kennedy e McNally 2005, p.371)

Já o advérbio much é um modificador que tem uma interpretação muito similar a de very. No entanto, much só pode ser utilizado com adjetivos que são fechados no seu grau mínimo, como ilustrado na sentença (206b) acima. Needed 'necessário' é um adjetivo de escala fechada em grau mínimo, ou seja, não há um grau máximo de ser necessário, mas há um grau mínimo. O teste feito para se certificar que um adjetivo possui uma escala fechada no seu grau mínimo é o seguinte:

(208) X não é A $=$ X não tem nenhuma A-dade (substantivo formado de A)

O que a fórmula em (208) quer dizer é que A é um adjetivo de escala fechada no grau mínimo se passar pelo seguinte teste de acarretamento: Se $X$ não é $A$ é verdadeira, então necessariamente $X$ não tem nenhuma A-dade é verdadeira. Por exemplo, necessário é um adjetivo de escala fechada em seu grau mínimo porque ele passa pelo teste em (209):

(209) X não é necessário (para Y) $=$ X não tem nenhuma necessidade (para Y)

Ou seja, se $\mathbf{X}$ não é necessário, isso acarreta que esse $\mathbf{X}$ não tem nenhuma necessidade. Outros adjetivos que passam pelo mesmo teste e que são, portanto predicados de escala fechada no grau mínimo são: criticized 'criticado', praised 'elogiado' e appreciated 'apreciado', conforme mostram os testes abaixo. Os testes foram propostos para os adjetivo em inglês. No entanto, esses adjetivos em português parecem ter o mesmo comportamento.

(210) a. $\quad X$ não foi criticado $=X$ não teve nenhuma crítica

a'. $\quad$ 'O artigo não foi criticado' $F$ ' $X$ não teve nenhuma crítica' 

b. $\quad \mathrm{X}$ não foi elogiado $=\mathrm{X}$ não teve nenhum elogio
b'. $\quad$ 'O artigo não foi elogiado' $=$ ' 'O artigo não teve nenhum elogio'
c. $\quad \mathrm{X}$ não foi apreciado $=\mathrm{X}$ não teve nenhum apreço
c'. ' $\quad$ artigo não foi apreciado' $=$ ' 'O artigo não teve nenhum apreço'

Por fim, well pode ser usado apenas com adjetivos de escalas totalmente fechadas. $\mathrm{O}$ dado (206c), repetido abaixo em (211), apresenta uma sentença com well e o adjetivo acquainted 'familiarizado'. Esse é um adjetivo absoluto porque uma vez que se está acquainted 'familiarizado' com algo, não é possível ficar ainda mais acquainted 'familiarizado', ou ainda, não é preciso se comparar outras situações de Martin Beck estar acquainted 'familiarizado' para atribuir-lhe essa qualidade ${ }^{85}$.

(211) Martin Beck was well (?much/?very) acquainted with the facts of the case. $=(206 \mathrm{c})$

'O Martin Beck estava bem familiarizado com os fatos do caso'

Uma característica singular de well que o diferencia de very e much é que ele relativiza o adjetivo, devolvendo, no fim da operação, um predicado relativo, ou seja, de escala aberta. Isso explica porque um adjetivo que já foi modificado por well pode ainda ser modificado por very, que só pode ocorrer com predicados relativos, como se vê em (212), abaixo. Aware 'consciente' é um adjetivo absoluto, de escala fechada, como mostra o exemplo em (212a). Por isso, well pode modificá-lo. O predicado resultante well aware 'bem consciente' tem, por sua vez, escala aberta (não há um grau máximo para se ficar bem consciente); ele pode, portanto, ser modificado por very.

(212) a. John is completely/fully aware of his responsability.

'O John está completamente/totalmente consciente de sua responsabilidade'

b. They remained very well aware of the difficulties that might arise from their analysis.

'Eles ficaram muito bem conscientes das dificuldades que poderiam surgir de sua análise'

(exemplo 89 de Kennedy e McNally 2005, p. 376)

\footnotetext{
Esta subseção é baseada nas afirmações de Kennedy e McNally (2005) para o inglês. Familiarizado em português parece não ter o mesmo comportamento de acquainted do inglês.
} 
Esta seção mostrou que a divisão dos predicados graduáveis em relativos e absolutos e de escala aberta e fechada é relevante para a análise dos modificadores de grau. Ela pode auxiliar na análise da distribuição e significado de modificadores de grau, como no caso adjetivos deverbais do inglês modificados por very, much e well

\subsection{Formalização}

O objetivo desta subseção é mostrar de que forma a Semântica Escalar vem tratando formalmente predicados graduáveis e modificadores de grau. A primeira grande mudança em relação a uma Semântica não escalar diz respeito ao tipo semântico d. Para a Semântica Escalar, além dos tipos tradicionais e para indivíduos, $\mathbf{s}$ para eventos e $\mathbf{t}$ para valores de verdade, o domínio semântico possui também um tipo d para graus ${ }^{86}$. Segundo a Semântica Escalar, adjetivos graduáveis denotam uma relação entre graus e indivíduos. O exemplo abaixo com o adjetivo caro ilustra a denotação de um adjetivo graduável segundo essa proposta.

$$
\llbracket \text { caro } \rrbracket=\lambda \mathrm{d}_{\mathrm{d}} \lambda \mathrm{x}_{\mathrm{e}} \cdot \text { “custo" }(\mathrm{x})=\mathrm{d}^{87}
$$

A denotação acima quer dizer que caro é um predicado que pega um argumento do tipo $\mathbf{d}$ e um argumento do tipo e e devolve o mapeamento do argumento $\mathbf{x}$ em um grau $\mathbf{d}$ em uma escala de custo. E esse grau $\mathbf{d}$ representa o custo de $\mathbf{x}$. $\mathrm{O}$ adjetivo tem tipo $<\mathrm{d},<\mathrm{e}, \mathrm{t}>>$. Em sua denotação tradicional, os adjetivos são predicados de indivíduos de tipo <e,t>. Segundo a denotação em (214), caro é um predicado que pega um argumento $\mathbf{x}$ de tipo e e devolve o valor de verdade 1, se $\mathbf{x}$ é caro. A questão composicional de como um adjetivo graduável de tipo $<\mathrm{d},<\mathrm{e}, \mathrm{t}>>$ será tranformado em um predicado de indivíduos de tipo $<\mathrm{e}, \mathrm{t}>$ será discuta mais abaixo.

(214) $\llbracket \operatorname{caro} \rrbracket=\lambda x$. $\operatorname{caro}(\mathrm{x})$

${ }_{87}^{86}$ Os tipos semânticos foram discutidos no capítulo 1.

87 Em Kennedy (1999) e Kennedy (2007), os adjetivos denotam diretamente funções de medida de tipo <e,d>. Um adjetivo como caro denotaria $\lambda x$. "custo" $(x)$ nessa proposta. Nesta tese estamos assumindo a proposta de Kennedy e McNally (2005) de que a função de medida faz parte da denotação do predicado. As dimensões das escalas estão sendo representadas entre aspas. 
$\mathrm{Na}$ denotação em (213), "custo" é uma função de medida que mapeia seus argumentos em representações abstratas (os graus nas escalas). Na denotação em (213), fíca claro de que forma é feita a associação entre indivíduos e graus descrita na seção subseção 2.2. Em 2.2, o exemplo utilizado foi alto. O indivíduo Luciana era associado ao grau 1,61 na escala "altura" mapeada em metros, enquanto que Oscar era mapeado no grau 2,05. As fórmulas abaixo mostram a aplicação da função de medida que gerou a escala descrita anteriormente $^{88}$. (215b) e (215c) representam a função de medida "altura" associada a Luciana e a Oscar respectivamente.

$$
\begin{aligned}
& \text { a. } \quad \text { alto } \rrbracket=\lambda \mathrm{d} \lambda \mathrm{x} \text {. "altura" }(\mathrm{x})=\mathrm{d} \\
& \text { b. "altura" (Luciana) }=1,61 \\
& \text { c. "altura" (Oscar) }=2,05
\end{aligned}
$$

Assim, uma generalização pode ser feita sobre a denotação dos adjetivos graduáveis:

$$
\llbracket A d j_{g} \rrbracket=\lambda \mathrm{d} \lambda \mathrm{x} . \mathrm{g}_{\mathrm{adj}}(\mathrm{x})=\mathrm{d}
$$

$\mathrm{Na}$ denotação acima, $\mathbf{g}_{\text {adj }}$ é a função de medida associada ao adjetivo. Na denotação do adjetivo alto, utilizado acima, a função de medida é altura. No caso dos adjetivos, essa função de medida é dada pelo léxico. O adjetivo alto é associado a uma função de medida de altura, o adjetivo pesado é associado a uma função de medida peso, o adjetivo cheio a uma função de medida ocupação e assim por diante.

Os predicados graduáveis de tipo $\langle\mathrm{d}<\mathrm{e}, \mathrm{t}\rangle>$ são convertidos em predicados de indivíduos de tipo $<\mathrm{e}, \mathrm{t}>$ por meio de uma morfologia de grau. A morfologia de grau pode ser, por exemplo, uma comparação, modificadores intensificadores ou de suficiência como muito e tão, respectivamente; ou ainda itens de pergunta como o how 'quão' do inglês, em perguntas do tipo how tall? 'quão alto?'. A morfologia de grau nesse caso prende a variável de grau representada por $\lambda \mathbf{d}$ na denotação do adjetivo.

Os casos em que não há uma morfologia de grau explícita são considerados como a forma positiva do adjetivo. A forma positiva do adjetivo é a predicação de um indivíduo por um adjetivo graduável, como na sentença abaixo.

\footnotetext{
88 Os valores de graus nem sempre são números naturais como no caso descrito. Em escalas não discretas como "felicidade" ou "beleza", os graus são pontos na escala, mas não são descritos numericamente.
} 
Nesses casos, não há morfologia explícita de grau para transformar o predicado de tipo $<\mathrm{d}<\mathrm{e}, \mathrm{t}>>$ em um predicado de tipo $<\mathrm{e}, \mathrm{t}>$. Há uma incompatibilidade de tipos, como mostra a tentativa de Aplicação Funcional abaixo.

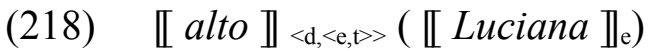

Uma proposta em potencial para que a predicação seja possível seria por meio de uma mudança de tipos (do inglês type shifting) do predicado alto de $<\mathrm{d}<\mathrm{e}, \mathrm{t}\rangle>$ para $<\mathrm{e}, \mathrm{t}\rangle$. Uma outra proposta possível é considerar a existência de um morfema de grau nulo que executa a função de prender a variável representada por $\boldsymbol{\lambda} \mathbf{d}$. Por uma questão de uniformidade com as formas de grau abertas como comparativas e modificadores, a teoria tem optado pela segunda alternativa (Kennedy e McNally 2005 e Kennedy 2007). Os autores deixam claro, no entanto, que não há grande diferença entre essa proposta e a implementação via mudança de tipos. Um argumento em favor dessa escolha seria encontrar uma língua que possuísse o morfema nulo pronunciado. No entanto, até o momento, não houve indícios de uma língua com essa característica.

O morfema de grau nulo é usualmente chamado de pos e tem a função de prender a variável de grau representada pelo $\lambda \mathbf{d}$, além de derivar o significado adequado das sentenças com os adjetivos graduáveis em sua forma positiva. A denotação de pos apresentada abaixo foi adaptada de Kennedy $(2007)^{89}$.

$$
\llbracket p o s \rrbracket=\lambda \mathrm{G}_{<\mathrm{d},<\mathrm{e}, \triangleright>} \lambda \mathrm{x}_{\mathrm{e}} . \exists \mathrm{d}\left[\mathrm{G}(\mathrm{d})(\mathrm{x}) \& \mathrm{~d} \geq \mathrm{d}_{\mathrm{s}}\right]
$$

$\mathrm{Na}$ denotação acima, $\mathbf{G}$ é a variável de adjetivos graduáveis de tipo $<\mathrm{d}<\mathrm{e}, \mathrm{t}>>$, $\mathbf{x}$ e d são as já conhecidas variáveis sobre indivíduos e graus (tipo e e d respectivamente) e $\mathbf{d}_{\mathrm{s}}$ é o grau apropriado segundo o parâmetro de comparação. Na proposta de Kennedy (2007) o grau $\mathbf{d}_{\mathrm{s}}$ é baseado tanto nas propriedades do adjetivo, quanto nas propriedades do contexto de enunciação. Se o predicado graduável for de escala aberta, $\mathbf{d}_{\mathbf{s}}$ representa o grau normal da escala. Se o predicado graduável for de escala fechada, $\mathbf{d}_{\text {s }}$ representa o grau máximo da

\footnotetext{
89 A denotação proposta por Kennedy (2007) foi adaptada porque o autor considera que os adjetivos denotam funções de medida de tipo $<$ e,d $>$ e não predicados de tipo $<$ d, $<$ e, $t>>$.
} 
escala. Os exemplos de composição abaixo mostram como esse grau é selecionado. A aplicação do morfema pos ao adjetivo relativo alto para a composição semântica da sentença (220) é apresentada em (221).

(220) A Luciana é alta.

$$
\begin{aligned}
& \text { a. } \llbracket \text { alto } \rrbracket=\lambda \mathrm{d} \lambda \mathrm{x} \text {. "altura" }(\mathrm{x})=\mathrm{d} \quad \text { Léxico } \\
& \text { b. } \quad \llbracket \text { alto }_{p o s} \rrbracket=\llbracket p o s \rrbracket(\llbracket \text { alto } \rrbracket) \quad \text { Aplicação Funcional } \\
& \text { c. } \llbracket \text { pos } \rrbracket(\llbracket \text { alto } \rrbracket)=\left[\lambda \mathrm{G} \lambda \mathrm{x} . \exists \mathrm{d}\left[\mathrm{G}(\mathrm{d})(\mathrm{x}) \& \mathrm{~d} \geq \mathrm{d}_{\mathrm{s}}\right]\right](\lambda \mathrm{d} \lambda \mathrm{x} \text {. "altura" }(\mathrm{x})=\mathrm{d}) \\
& \text { d. } \llbracket \text { alto }_{p o s} \rrbracket=\lambda \mathrm{x} . \exists \mathrm{d}\left[[\lambda \mathrm{d} \lambda \mathrm{x} \text {. "altura" (x) }=\mathrm{d}](\mathrm{d})(\mathrm{x}) \& \mathrm{~d}>\mathrm{d}_{\mathrm{s}}\right]^{90} \\
& =\lambda \mathrm{x} . \exists \mathrm{d}\left[\text { "altura" }(\mathrm{x})=\mathrm{d} \& \mathrm{~d}>\mathrm{d}_{\mathrm{s}}\right] \\
& \text { e. } \llbracket \text { alto }_{p o s} \rrbracket(\llbracket \text { Luciana } \rrbracket)^{91}=\left[\lambda \mathrm{x} . \exists \mathrm{d}\left[\text { [“altura” }(\mathrm{x})=\mathrm{d} \& \mathrm{~d}>\mathrm{d}_{\mathrm{s}}\right]\right](\text { Luciana }) \\
& \text { Aplicação Funcional } \\
& =\exists \mathrm{d}\left[\text { "altura" (Luciana) }=\mathrm{d} \& \mathrm{~d}>\mathrm{d}_{\mathrm{s}}\right] \\
& \text { f. } \quad \llbracket \text { A Luciana é alta } \rrbracket^{92}=1 \text { sse } \exists d \text { [ "altura" (Luciana) }=\mathrm{d} \& \mathrm{~d}>\mathrm{d}_{\mathrm{s}} \text { ] }
\end{aligned}
$$

A denotação em (221f) mostra que a sentença $A$ Luciana é alta é verdadeira se existe um grau d, tal que o indivíduo Luciana está associado a esse grau d na escala de altura, e esse grau $\mathbf{d}$ é maior do que o grau $\mathbf{d}_{\mathrm{s}}$ determinado levando-se em conta a função de medida altura e o parâmetro de comparação contextual. O grau d d é o grau que designamos como grau normal na descrição dos adjetivos relativos na seção 2.2. Ele é o grau que representa em um contexto o que é considerado o ponto de corte da escala (do inglês cutoff point). Abaixo dele os indivíduos não são considerados altos, e acima dele são.

Apresentamos agora um exemplo de derivação de uma sentença com um adjetivo absoluto em sua forma positiva.

$$
\text { O copo está cheio. }
$$

90 Uma vez que se trata de um adjetivo de escala aberta, o $\geq$ é substituído por $>$ para garantir que o grau $\mathbf{d}$ seja maior que o grau $\mathbf{d}_{\mathbf{s}}$, grau normal da escala. Quando o adjetivo é de escala fechada, o $\geq$ é substituído por $=$.

91 A concordância é um fenômeno sintático e não é considerado na computação semântica.

92 O verbo de ligação é semanticamente vácuo e não é considerado na computação semântica. O mesmo vale para o artigo definido antes de nomes próprios. 


$$
\begin{aligned}
& \text { a. } \llbracket \text { cheio } \rrbracket=\lambda \mathrm{d} \lambda \mathrm{x} \text {. "ocupação" }(\mathrm{x})=\mathrm{d} \quad \text { Léxico } \\
& \text { b. } \quad \llbracket \text { cheio }_{p o s} \rrbracket=\llbracket \text { pos } \rrbracket(\llbracket \text { cheio } \rrbracket) \quad \text { Aplicação Funcional } \\
& \text { c. } \llbracket \text { 【pos } \rrbracket(\llbracket \text { cheio } \rrbracket)=\left[\lambda \mathrm{G} \lambda \mathrm{x} . \exists \mathrm{d}\left[\mathrm{G}(\mathrm{d})(\mathrm{x}) \& \mathrm{~d} \geq \mathrm{d}_{\mathrm{s}}\right]\right] \\
& (\lambda \mathrm{d} \lambda \mathrm{x} \text {. "ocupação" }(\mathrm{x})=\mathrm{d}) \\
& \text { d. } \llbracket \text { cheio }_{p o s} \rrbracket=\lambda \mathrm{x} . \exists \mathrm{d}\left[[\lambda \mathrm{d} \lambda \mathrm{x} \text {. "ocupação" }(\mathrm{x})=\mathrm{d}](\mathrm{d})(\mathrm{x}) \& \mathrm{~d}=\mathrm{d}_{\mathrm{s}}\right] \\
& =\lambda \mathrm{x} . \exists \mathrm{d}\left[\text { "ocupação" }(\mathrm{x})=\mathrm{d} \& \mathrm{~d}=\mathrm{d}_{\mathrm{s}}\right] \\
& (\lambda x \cdot \operatorname{copo}(x)) \\
& =\mathrm{o} \text { único } \mathrm{x} \text { no contexto tal que copo(x) }
\end{aligned}
$$

Aplicação Funcional

$$
\begin{aligned}
&= {\left[\lambda \mathrm{x} \exists \mathrm{d} . \text { "ocupação" }(\mathrm{x})=\mathrm{d} \& \mathrm{~d}=\mathrm{d}_{\mathrm{s}}\right] } \\
&(\mathrm{o} \text { único x no contexto tal que copo }(\mathrm{x})) \\
&= 1 \text { sse } \exists \mathrm{d}[\text { "ocupação" (o único x no contexto tal que } \\
&\left.\left.\operatorname{copo}(\mathrm{x}))=\mathrm{d} \& \mathrm{~d}=\mathrm{d}_{\mathrm{s}}\right]\right]
\end{aligned}
$$

A denotação em (223i) representa que a denotação da sentença $O$ copo está cheio é o valor de verdade 1 se existe um grau d, tal que o único indivíduo que é copo no contexto está associado a esse grau $\mathbf{d}$ na escala de ocupação, e esse grau $\mathbf{d}$ é igual ao grau $\mathbf{d}_{\mathbf{s}}$ determinado levando-se em conta a função de medida ocupação e o parâmetro de comparação contextual. Uma vez que agora se trata de um adjetivo de escala fechada, o grau $\mathbf{d}_{\mathbf{s}}$ nesse caso não é o grau normal, mas é o grau máximo da escala. Segundo Kennedy (2007), isso se dá graças a um princípio chamado Economia Interpretativa, definido a seguir.

\footnotetext{
93 A denotação do artigo definido é baseada em Heim e Kratzer (1998). Ela foi adaptada e não está levando em consideração questões como a pressuposição de existência e unicidade. Ver Heim e Kratzer (1998) para mais detalhes.
} 
(224) Economia Interpretativa:

Maximize a contribuição do significado convencional dos elementos da sentença para a computação das suas condições de verdade.

O efeito da Economia Interpretativa é assegurar que os adjetivos de escala fechada como cheio sejam interpretados como adjetivos absolutos na forma positiva. O princípio da Economia Interpretativa exige que o grau máximo da escala (que faz parte do significado do adjetivo) seja computado na escolha do grau $\mathbf{d}_{\mathbf{s}}$ da forma positiva do adjetivo.

Assim como no caso dos adjetivos relativos, na forma positiva do adjetivos absolutos, o grau d também é considerado o grau que representa o ponto de corte da escala. Indivíduos que atingem o grau $\mathbf{d}_{\mathbf{s}}$ são considerados cheios, e os que não atingem, não são. Essa noção de grau que representa o ponto de corte será importante para a análise da modificação de grau em Karitiana apresentada abaixo.

\section{$3 \quad$ Modificação de Grau em Karitiana}

O objetivo desta seção é apresentar a distribuição do modificador de grau pita(t) com adjetivos, verbos e sintagmas nominais. A seção começa apresentando primeiramente uma discussão acerca da distribuição de pita e pitat mostrando porque o morfema $\{-t\}$ vem sendo identificado como adverbializador neste trabalho. Pita não está sendo traduzido nas glosas porque não há em português uma palavra que expresse o conteúdo semântico de pita. Seu significado será amplamente discutido durante o capítulo. A seção 3.2 apresenta a distribuição do modificador com adjetivos. A análise começa com adjetivos porque, conforme foi apresentado nas seções anteriores, a Semântica Escalar tem uma análise bem estabelecida para a modificação dessa classe de palavras. Em seguida, a seção 3.3 apresenta a distribuição e análise de pita(t) com sintagmas verbais. A seção 3.4 mostra, por fim, o uso desse modificador como adjetivo quando aplicado a sintagmas nominais. 


\subsection{Pita vs Pitat}

O objetivo desta seção é apresentar a distribuição de pita e de pitat justificando porque o morfema $\{-t\}$ vem sendo glosado como adverbializador. A distribuição de pita e de pitat é uniforme: pita é usado quando está modificando adjetivos ou sintagmas nominais e pitat é usado quando está modificando predicados verbais. Os exemplos de (225a) a (225c) mostram pita dentro de um sintagma nominal modificando um substantivo. $\mathrm{O}$ significado dessas sentenças será análisado em detalhes na seção 3.4. Nesta seção, vamos nos concentrar na distribuição morfossintática.
a. [Taso pita] i-otam-Ø.
homem pita PART-chegar-ABS
'O homem verdadeiro chegou'
b. Elivar i-amy-t [gooj pita].
Elivar PART-comprar-ABS carro pita
'O Elivar comprou um carro verdadeiro'

Marilena i-amy-t [opi pita].
Marilena PART-comprar-ABS brinco pita
'A Marilena comprou um brinco verdadeiro'

As sentenças abaixo mostram o uso de pita modificando adjetivos dentro do sintagma nominal.

(226) a. [Õwã se'a pita] i-otam-Ø.

menino bom pita PART-chegar-ABS

'O menino muito bom chegou'

b. [Õwã ty pita] i-otam-Ø.

menino grande pita PART-chegar-ABS

'O menino muito grande chegou' 
c. [Õwã siin pita] i-otam-Ø.

menino pequeno pita PART-chegar-ABS

O menino muito pequeno chegou'

As sentenças abaixo mostram a distribuição de pitat modificando predicados verbais.

(227) a. João i-pytim'adn-<a>-t pita-t.

João PART-trabalhar-ABS pita-ADV

'O João trabalhou muito'

b. Inácio i-pykyn- $<\mathrm{a}>-\mathrm{t} \quad$ pita-t.

Inácio PART-correr-ABS pita-ADV

'O Inácio correu muito'

c. João i-kywytidn- $<\mathrm{a}>-\mathrm{t}$ pita-t Botỹj-ty.

João PART-acreditar-ABS pita-ADV Deus-OBL

'O João acredita muito em em Deus'

A distribuição apresentada acima mostra que o sufixo $\{-t\}$ é exclusivo do uso de pita(t) modificando predicados verbais. É por conta dessa distribuição que ele está sendo glosado como adverbializador neste trabalho. Muitos outros advérbios possuem o mesmo morfema. Kandat(t) utilizado no capítulo 2 desta tese é um exemplo, como mostra a sentença abaixo.

jonso $\varnothing$-naka-ot- $\varnothing \quad$ kanda-t

ese. $=(90)=(94)$

mulher 3-DECL-buscar-NFUT muitas.vezes-ADV água

'A mulher buscou água muitas vezes'

As sentenças abaixo mostram outros advérbios que também possuem um sufixo $\{-t\}$ em Karitiana. 
(229)

a. João i-otam-Ø koo-t.

João PART-chegar-ABS ontem-ADV

'O João chegou ontem'

b. João Ø-naka-tagngã-t gooj soaso-t.

João 3-DECL-dirigir-NFUT carro rápido-ADV

'O João dirige rápido o carro'

$\begin{array}{lll}\text { c. } & \text { João } \text { i-otam- } \varnothing & \text { kama-t. } \\ \text { João } & \text { PART-chegar-ABS } & \text { agora-ADV } \\ \text { 'O João chegou agora' } & \end{array}$

Rocha (2013) mostrou que as orações subordinadas adverbiais também possuem um sufixo $\{-t\}$ adverbializador.

(230) [Gok jonso amang-ã tyki'oo-t] Ø-na-oky-t him taso.

[macaxeira mulher plantar-VT IMP-ADV] 3-DECL-matar-NFUT caça homem

'Enquanto a mulher estava plantando macaxeira, o homem matou a caça'

(exemplo 31 de Rocha 2013, p. 6)

Esta seção mostrou a distribuição de pita(t) modificando substantivos, adjetivos e verbos que justifica a glosa de advebializador para o sufixo $\{-t\}$ empregada nesta tese. As próximas seções vão tratar da distribuição e significado com cada uma dessas classes. Em seguida, apresentamos uma proposta unificada para o modificador. 


\subsection{Adjetivos}

O objetivo desta seção é apresentar a distribuição de pita modificando sintagmas adjetivais. A tabela abaixo mostra os adjetivos utilizados para os testes de pita com adjetivos. Foram selecionados os adjetivos que são tradicionalmente considerados em cada classe. Ao longo da seção, fica claro que essa classificação é valida também em Karitiana. Mais do que isso ela será de suma importância para a análise que vamos defender.

Tabela 38: Adjetivos Analisados

\begin{tabular}{|c|c|}
\hline Adjetivo & Tradução \\
\hline \multicolumn{2}{|c|}{ Adjetivos de Escalas Abertas } \\
\hline se'a & bom \\
\hline sara'i & ruim \\
\hline ty & grande \\
\hline siin & pequeno \\
\hline pyti & pesado \\
\hline pawak & leve \\
\hline \multicolumn{2}{|c|}{ Adjetivos de Escalas Totalmente Fechadas } \\
\hline akyndop & aberto \\
\hline akydnop & fechado \\
\hline osyk & cheio \\
\hline piyywyp & vazio \\
\hline \multicolumn{2}{|c|}{ Adjetivos de Escala Parcialmente Fechadas } \\
\hline pok & seco \\
\hline sembok & molhado \\
\hline sara'i & perigoso \\
\hline se'a & seguro \\
\hline \multicolumn{2}{|c|}{ Adjetivos não Graduáveis } \\
\hline grávida & 'edna \\
\hline
\end{tabular}


Uma vez que os adjetivos canonicamente não graduáveis são normalmente os que envolvem conceitos matemáticos, como hexagonal e primo, ou científico como extinto, tivemos dificuldade para encontrar palavras da língua que expressassem uma propriedade não graduável. O único adjetivo não graduável que conseguimos testar em Karitiana foi 'edna 'grávida'. O quadro acima mostra também que não há adjetivos específicos para seguro e perigoso. São usadas as mesmas palavras para bom e ruim.

Uma evidência de que os adjetivos graduáveis selecionados são mesmo graduáveis em Kariana é a sua possibilidade de uso em sentenças comparativas. A tabela mostra o uso dos adjetivos em sentenças comparativas. Em Karitiana, há muitos modos se fazer uma sentença comparativa. A mais comum delas é com um verbo de cópula e com a palavra ohynym que é semelhante ao mais do português. No entanto, ohynym também quer dizer 'maior', conforme mostra a tabela abaixo.

Tabela 39: Sentenças Comparativas com Adjetivos

\begin{tabular}{|c|c|}
\hline Adjetivo & Exemplo \\
\hline \multicolumn{2}{|r|}{ Adjetivos de Escalas Abertas } \\
\hline $\begin{array}{l}s e^{\prime} a \\
\text { 'bom' }\end{array}$ & 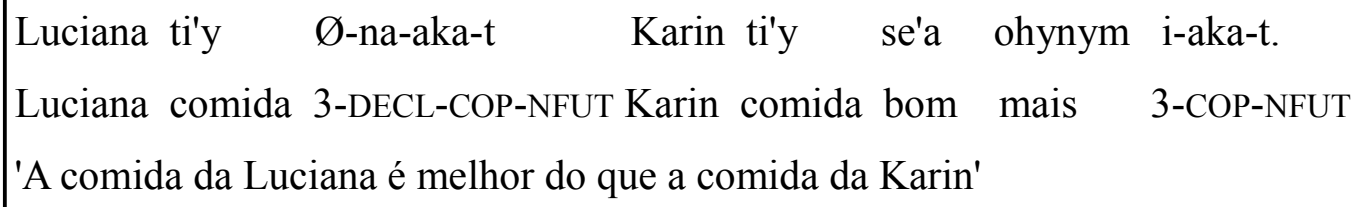 \\
\hline $\begin{array}{l}\text { sara'i } \\
\text { 'ruim' }\end{array}$ & $\begin{array}{lllll}\text { Luciana ti'y } & \varnothing \text {-na-aka-t } & \text { Karin ti'y sara'i } & \text { ohynym } & \text { i-aka-t. } \\
\text { Luciana comida } & \text { 3-DECL-COP-NFUT Karin comida ruim } & \text { mais } & \text { 3-COP-NFUT } \\
\text { 'A comida da Luciana é pior do que a comida da Karin' } & & \end{array}$ \\
\hline $\begin{array}{l}\text { ty } \\
\text { 'grande' }\end{array}$ & $\begin{array}{l}\text { Ismael } \varnothing \text {-na-aka-t Isaias ohynym i-aka-t. } \\
\text { Ismael 3-DECL-COP-NFUT Isaias maior } 3 \text {-COP-NFUT } \\
\text { 'O Ismael é maior do que o Isaias' }\end{array}$ \\
\hline $\begin{array}{l}\text { siin } \\
\text { 'pequeno' }\end{array}$ & $\begin{array}{llll}\text { Ismael } & \varnothing \text {-na-aka-t } \quad \text { Isaias ejep } \quad \text { i-aka-t. } \\
\text { Ismael } & 3 \text {-DECL-COP-NFUT Isaias menor } & \text { 3-COP-NFUT } \\
\text { 'O Ismael é menor do que o Isaias' } & \\
\end{array}$ \\
\hline $\begin{array}{l}\text { pyti } \\
\text { 'pesado' }\end{array}$ & $\begin{array}{l}\text { Luciana ombi } \varnothing \text {-na-aka-t Sarita ombi pyti ohynym } \text { i-aka-t. } \\
\text { Luciana cesto } 3 \text {-DECL-COP-NFUT Sarita cesto pesado mais } \\
\text { 3-COP-NFUT } \\
\text { 'O cesto da Luciana está mais pesado do que o cesto da Sarita' }\end{array}$ \\
\hline
\end{tabular}




\begin{tabular}{|c|c|}
\hline $\begin{array}{l}\text { pawak } \\
\text { 'leve' }\end{array}$ & $\begin{array}{l}\text { Luciana ombi } \varnothing \text {-na-aka-t Sarita ombi pawak ohynym i-aka-t. } \\
\text { Luciana cesto } 3 \text {-DECL-COP-NFUT Sarita cesto leve mais } \\
\text { 3-COP-NFUT } \\
\text { 'O cesto da Luciana está mais leve do que o cesto da Sarita' }\end{array}$ \\
\hline \multicolumn{2}{|r|}{ Adjetivos de Escalas Totalmente Fechadas } \\
\hline $\begin{array}{l}\text { akyndop } \\
\text { 'aberto' }\end{array}$ & $\begin{array}{l}\text { Funai karamã } \varnothing \text {-na-aka-t Associação karamã akyndop ohynym i-aka-t. } \\
\text { Funai porta } 3 \text {-DECL-COP-NFUT Associação porta aberto mais } 3 \text {-COP-NFUT } \\
\text { 'A porta da Funai está mais aberta do que a porta da Associação' }\end{array}$ \\
\hline $\begin{array}{l}\text { akydnop } \\
\text { 'fechado' }\end{array}$ & $\begin{array}{l}\text { Funai karamã } \varnothing \text {-na-aka-t Associação karamã akydnop ohynym i-aka-t. } \\
\text { Funai porta } 3 \text {-DECL-COP-NFUT Associação porta fechado mais 3-COP-NFUT } \\
\text { 'A porta da Funai está mais fechada do que a porta da Associação' }\end{array}$ \\
\hline $\begin{array}{l}\text { osyk } \\
\text { 'cheio' }\end{array}$ & $\begin{array}{l}\text { Luciana ombi } \varnothing \text {-na-aka-t Sarita ombi osyk ohynym i-aka-t. } \\
\text { Luciana cesto } 3 \text {-DECL-COP-NFUT Sarita cesto cheio mais } 3 \text {-COP-NFUT } \\
\text { 'O cesto da Luciana está mais cheio do que o cesto da Sarita' }\end{array}$ \\
\hline $\begin{array}{l}\text { piyywyp } \\
\text { 'vazio' }\end{array}$ & 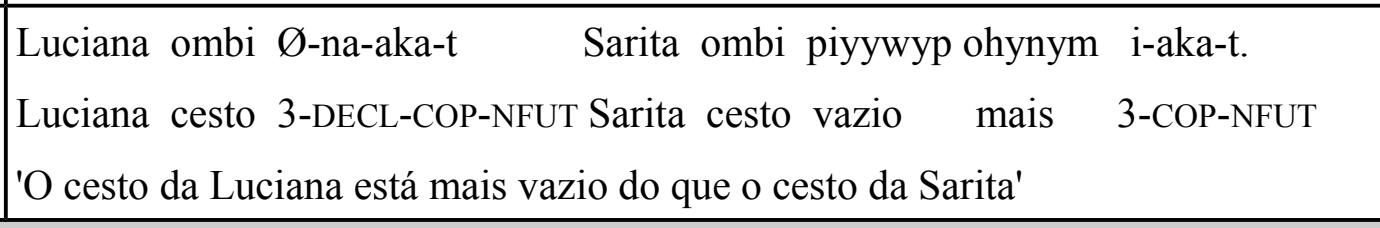 \\
\hline \multicolumn{2}{|r|}{ Adjetivos de Escala Parcialmente Fechadas } \\
\hline $\begin{array}{l}\text { pok } \\
\text { 'seco' }\end{array}$ & $\begin{array}{l}\text { Luciana pykyp Ø-na-aka-t Karin pykyp pok ohynym i-aka-t. } \\
\text { Luciana roupa } 3 \text {-DECL-COP-NFUT Karin roupa seco mais } 3 \text {-COP-NFUT } \\
\text { 'A roupa da Luciana está mais seca do que a roupa da Karin' }\end{array}$ \\
\hline $\begin{array}{l}\text { sembok } \\
\text { 'molhado' }\end{array}$ & $\begin{array}{l}\text { Luciana pykyp Ø-na-aka-t } \quad \text { Karin pykyp sembok ohynym i-aka-t. } \\
\text { Luciana roupa } 3 \text {-DECL-COP-NFUT Karin roupa molhado mais } 3 \text {-COP-NFUT } \\
\text { 'A roupa da Luciana está mais molhada do que a roupa da Karin' }\end{array}$ \\
\hline $\begin{array}{l}\text { sara'i } \\
\text { 'perigoso' }\end{array}$ & 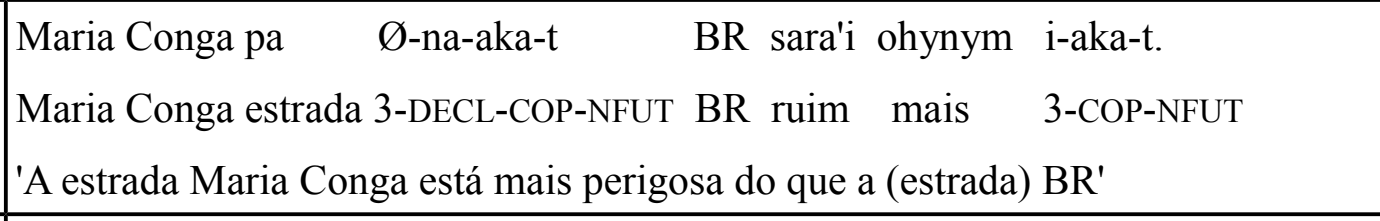 \\
\hline $\begin{array}{l}s e^{\prime} a \\
\text { 'seguro' }\end{array}$ & $\begin{array}{l}\text { Maria Conga pa } \quad \text {-na-aka-t BR se'a ohynym i-aka-t. } \\
\text { Maria Conga estrada 3-DECL-COP-NFUT BR bom mais } 3 \text {-COP-NFUT } \\
\text { 'A estrada Maria Conga está mais segura do que a (estrada) BR' }\end{array}$ \\
\hline
\end{tabular}




\begin{tabular}{|c|c|}
\hline \multicolumn{2}{|r|}{ Adjetivos não Graduáveis } \\
\hline 'edna & Karin 'edna ohynym i-aka-t. \\
\hline 'grávida' & Luciana 3-DECL-COP-NFUT Karin grávida mais \\
\hline & 'A Luciana está mais grávida do que a Karin' \\
\hline & Situação: grávida há mais tempo \\
\hline
\end{tabular}

O quadro acima mostrou que apenas predicados graduáveis em Karitiana podem aparecer em uma estrutura comparativa com o significado de comparação das propriedades que denotam. Embora a sentença comparativa com o adjetivo 'edna 'grávida' seja possível na língua, sua leitura é ligeiramente modificada. O que se está comparando é o tempo de gravidez e não propriamente a propriedade de estar grávida. Conforme foi apresentado na seção 2.1 essa mudança é prevista. Em português e em inglês, quando se diz que alguém é mais vegetariano do que outra pessoa ou está mais grávida, há uma coerção para que se considere, por exemplo, o tempo que os indivídios são vegetarianos ou estão grávidas. $\mathrm{O}$ fato de em Karitiana essa coerção também ocorrer indica que esse adjetivo também não é graduável na língua.

A evidência de que faz sentido separar os adjetivos de escalas abertas e (totalmente ou parcialmente) fechadas em Karitiana vem da própria distribuição de pita que será discutida a seguir. A tabela abaixo mostra que pita pode ser usado tanto com adjetivos de escalas abertas, os relativos, quanto com adjetivos de escala (totalmente ou parcialmente) fechadas, os absolutos. No entanto, seu significado varia um pouco em cada contexto, o que aparece na tradução. Os dados da tabela mostram que quando é usado para modificar os adjetivos de escalas abertas como $s e^{\prime} a$ 'bom' e ty 'grande', pita tem um comportamento parecido com o muito do português, ele seleciona um grau acima do normal associada à escala do adjetivo. 
Tabela 40: Adjetivos de Escala Aberta Modificados por Pita

\begin{tabular}{|c|c|}
\hline Adjetivo & Exemplo \\
\hline \multicolumn{2}{|r|}{ Adjetivos de Escalas Abertas } \\
\hline $\begin{array}{l}s e^{\prime} a \\
\text { 'bom' }\end{array}$ & $\begin{array}{l}\text { Õwã se'a pita i-otam- } \varnothing . \\
\text { menino bom pita PART-chegar-ABS } \\
\text { 'O menino muito bom chegou' }\end{array}$ \\
\hline $\begin{array}{l}\text { sara'i } \\
\text { 'ruim' }\end{array}$ & $\begin{array}{l}\text { Õwã sara'i pita i-otam- } \varnothing . \\
\text { menino ruim pita PART-chegar-ABS } \\
\text { 'O menino muito ruim chegou' }\end{array}$ \\
\hline $\begin{array}{l}\text { ty } \\
\text { 'grande' }\end{array}$ & $\begin{array}{l}\text { Õwã ty pita i-otam- } \varnothing . \\
\text { menino grande pita PART-chegar-ABS } \\
\text { 'O menino muito grande chegou' }\end{array}$ \\
\hline $\begin{array}{l}\text { siin } \\
\text { 'pequeno' }\end{array}$ & $\begin{array}{l}\text { Õwã siin pita i-otam- } \varnothing . \\
\text { menino pequeno pita PART-chegar-ABS } \\
\text { 'O menino muito pequeno chegou' }\end{array}$ \\
\hline $\begin{array}{l}\text { pyti } \\
\text { 'pesado' }\end{array}$ & $\begin{array}{l}\text { Ombi pyti pita i-ywym- } \varnothing \text {. } \\
\text { cesto pesado pita PART-sumiu-ABS } \\
\text { 'O cesto muito pesado sumiu' }\end{array}$ \\
\hline $\begin{array}{l}\text { pawak } \\
\text { 'leve' }\end{array}$ & $\begin{array}{l}\text { Ombi pawak pita i-ywym- } \varnothing \text {. } \\
\text { cesto leve pita PART-sumiu-ABS } \\
\text { 'O cesto muito leve sumiu' }\end{array}$ \\
\hline
\end{tabular}

Por outro lado, a tabela abaixo mostra que, quando pita é utilizado com adjetivos de escalas fechadas, como akyndop 'aberto' e akydno 'fechado', ele seleciona o grau máximo da escala e tem um significado parecido com o completamente do português. Com adjetivos de escala parcialmente fechadas, pita tem um significado de completamente com o adjetivo que representa o polo fechado da escala, como pok 'seco'. Com o adjetivo que representa o polo aberto da escala, ele tem o significado de muito. Esse comportamento representa exatamente a distribuição dos adjetivos very e completely descrita em Kennedy e McNally (2005). 
(231) a. The table is completely dry.

'A mesa está completamente seca'

b. ? The table is completely wet.

'A mesa está completamente molhada'

c. The table is very wet.

'A mesa está muito molhada'

(exemplos baseados em Kennedy e McNally 2005)

Tabela 41: Adjetivos de Escala Fechada Modificados por Pita

\begin{tabular}{|c|c|}
\hline Adjetivo & Exemplo \\
\hline \multicolumn{2}{|r|}{ Adjetivos de Escalas Totalmente Fechadas } \\
\hline $\begin{array}{l}\text { akyndop } \\
\text { 'aberto' }\end{array}$ & $\begin{array}{l}\text { Karamã akyndop pita i-pot-Ø. } \\
\text { porta aberto pita PART-quebrar-ABS } \\
\text { 'A porta completamente aberta quebrou' }\end{array}$ \\
\hline $\begin{array}{l}\text { akydnop } \\
\text { 'fechado' }\end{array}$ & $\begin{array}{l}\text { Karamã akydnop pita i-pot- } \varnothing \text {. } \\
\text { porta fechado pita PART-quebrar-ABS } \\
\text { 'A porta completamente fechada quebrou' }\end{array}$ \\
\hline $\begin{array}{l}\text { osyk } \\
\text { 'cheio' }\end{array}$ & $\begin{array}{l}\text { Ombi osyk pita i-ywym- } \varnothing \text {. } \\
\text { cesto cheio pita PART-sumiu-ABS } \\
\text { 'O cesto completamente cheio sumiu' }\end{array}$ \\
\hline $\begin{array}{l}\text { piyywyp } \\
\text { 'vazio' }\end{array}$ & $\begin{array}{l}\text { Ombi piyywyp pita i-ywym-Ø. } \\
\text { cesto vazio pita PART-sumiu-ABS } \\
\text { 'O cesto completamente vazio sumiu' }\end{array}$ \\
\hline \multicolumn{2}{|r|}{ Adjetivos de Escala Parcialmente Fechadas } \\
\hline $\begin{array}{l}\text { pok } \\
\text { 'seco' }\end{array}$ & $\begin{array}{l}\text { Pykyp pok pita i-ywym- } \varnothing \text {. } \\
\text { Roupa seco pita PART-sumiu-ABS } \\
\text { 'A roupa completamente seca sumiu' }\end{array}$ \\
\hline $\begin{array}{l}\text { sembok } \\
\text { 'molhado' }\end{array}$ & $\begin{array}{l}\text { Pykyp sembok pita i-ywym- } \varnothing \text {. } \\
\text { roupa molhada pita PART-sumiu-ABS } \\
\text { 'A roupa muito molhada sumiu' }\end{array}$ \\
\hline
\end{tabular}




\begin{tabular}{|c|c|}
\hline $\begin{array}{l}\text { sara'i } \\
\text { 'perigoso' }\end{array}$ & $\begin{array}{l}\text { João Ø-naka-typ-Ø } \quad \text { pa } \quad \text { sara'i pita. } \\
\text { João 3-DECL-encontrar-NFUT } \\
\text { 'O João encontrada ruim pita } \\
\text { 'O estrada muito perigosa' }\end{array}$ \\
\hline$s e^{\prime} a$ 'seguro' & $\begin{array}{l}\text { João Ø-naka-typ-Ø } \quad \text { pa se'a pita. } \\
\text { João 3-DECL-encontrar-NFUT } \\
\text { estrada bom pita } \\
\text { 'O João encontrou a estrada completamente segura' }\end{array}$ \\
\hline
\end{tabular}

Os dados acima mostraram que pita tem um comportamento dependente do tipo de predicado adjetival que modifica. Quando ocorre com adjetivos de escala aberta, ele determina um grau acima do normal da escala associada ao adjetivo. Nesses casos, ele é traduzido para o muito do português. Já quando ocorre com adjetivos de escala fechada, pita seleciona o grau máximo da escala e é traduzido por completamente nessas sentenças. Essa distribuição de pita indica que há uma certa semelhança entre o grau normal associado a adjetivos de escalas abertas e o grau máximo de adjetivos de escalas fechadas.

Essa semelhança não é surpreendente e aparece também em sentenças com adjetivos escalares não modificadas. No par de sentenças abaixo, embora as sentenças apresentem adjetivos associados a escalas de natureza diferentes (232a é de escala aberta e 232b de escala fechada), a predicação nos dois casos ocorre de modo bastante semelhante.

a. Ombi Ø-na-aka-t i-ty-t.

cesto 3-DECL-COP-NFUT PART-grande-ABS

'O cesto é grande'

b. Ombi Ø-na-aka-t i-osyk-

cesto 3-DECL-COP-NFUT PART-cheio-ABS

'O cesto está cheio'

Como a seção 2.4 mostrou, essa predicação se dá por meio do morfema de grau nulo pos. Sua denotação está reptida abaixo em (233).

$$
\llbracket \operatorname{pos} \rrbracket=\lambda \mathrm{G} \lambda \mathrm{x} . \exists \mathrm{d}\left[\mathrm{G}(\mathrm{d})(\mathrm{x}) \& \mathrm{~d} \geq \mathrm{d}_{\mathrm{s}}\right]
$$


Conforme apresentado acima, o grau $\mathbf{d}_{\mathbf{s}}$ está associado ao grau normal da escala quando pos se aplica a um adjetivo de escala aberta, como alto ou grande, e está associado ao grau máximo de uma escala quando é aplicado a um adjetivo de escala fechada, como cheio e fechado. Esses graus representam o ponto de corte em cada tipo de adjetivo, ou seja aquele grau que representa o ponto que determina quais indivíduos recebem ou não a predicação do adjetivo.

As tabelas acima mostraram que esse é exatamente o grau que pita seleciona quando modifica adjetivos. Quando modifica adjetivos de escalas abertas, pita indica um grau acima do grau normal da escala, e quando modifica adjetivos de escalas fechadas, seleciona o grau máximo da escala. Nesse sentido a entrada lexical que parece revelar adequadamente o significado de pita é a seguinte:

$$
\llbracket \text { pita } \rrbracket=\lambda \mathrm{G} \lambda \mathrm{x} . \exists \mathrm{d}\left[\mathrm{G}(\mathrm{d})(\mathrm{x}) \& \mathrm{~d} \geq \mathrm{d}_{\mathrm{s}}\right]
$$

No entanto, essa é exatamente a entrada lexical do morfema pos apresentado anteriormente, baseado em Kennedy (2007). O problema que emerge dessa semelhança está associado a uma questão que está sempre presente na análise dos modificadores de grau que pode ser exemplificada da seguinte forma: qual a diferença entre $O$ João é alto e $O$ João é muito alto? Se alto em sua forma positiva já significa acima de um grau normal na escala de altura, qual seria a contribuição de muito nesse caso? Segundo Kennedy e McNally (2005), essa diferença não aparece na denotação do modificador: o advérbio very 'muito' do inglês tem a mesma entrada lexical do morfema pos. A diferença, segundo os autores, está no parâmetro de comparação que é aumentado na denotação de very em relação ao morfema pos. A diferença de julgamento das sentenças abaixo estaria no fato de que na classe de comparação assumida em (235a) há indivíduos altos e baixos e na classe de comparação assumida para a avaliação da sentença $(235 b)$ haveria apenas indivíduos altos.

(235) a. John is tall.

'O John é alto'

b. John is very tall.

'O John é muito alto' 
Por questão de comparação, apresentamos abaixo a proposta de Kennedy e McNally (2005) para o morfema pos e para o modificador very. Os autores usam uma outra versão do morfema pos, representada em (236). Vamos continuar usando a versão do morfema descrita acima. A definição em (236) está sendo apresentada apenas para comparação da proposta de Kennedy e McNally (2005) para o very do inglês.

$$
\llbracket p o s \rrbracket=\lambda \mathrm{G} \lambda \mathrm{x} . \exists \mathrm{d}[\operatorname{standard}(\mathrm{d})(\mathrm{G})(\mathrm{C}) \& \mathrm{G}(\mathrm{d})(\mathrm{x})]
$$

(denotação 13 de Kennedy e McNally 2005, p.350)

$\mathrm{Na}$ proposta dos autores, há uma relação padrão (do inglês standard) entre o grau preso existencialmente, o predicado $\mathbf{G}$ e a classe de comparação que é representada por uma variável livre $\mathbf{C}$, que é determinada contextualmente. A entrada lexical de very 'muito' é a mesma de pos, com a diferença de que a forma positiva do adjetivo preenche a variável $\mathbf{C}$ de classe de comparação. Isso quer dizer que quando se diz very tall 'muito alto', a classe de comparação considerada é a dos indivíduos altos.

$$
\llbracket \operatorname{very} \rrbracket^{\mathrm{C}}=\lambda \mathrm{G} \lambda \mathrm{x} \cdot \exists \mathrm{d}\left[\operatorname{standard}(\mathrm{d})(\mathrm{G})\left(\lambda \mathrm{y} \cdot \llbracket \operatorname{pos}(\mathrm{G})(\mathrm{y}) \rrbracket^{\mathrm{C}}\right) \& \mathrm{G}(\mathrm{d})(\mathrm{x})\right]
$$
(denotação 64 de Kennedy e McNally 2005, p.370)

No entanto, estamos assumindo, nesta tese, a proposta de Kennedy (2007) para o morfema pos, em que o parâmetro de comparação e a classe de comparação não são representados na forma de variáveis livres na denotação de pos, mas são traduzidos na forma do grau $\mathbf{d}_{\mathbf{s}}$ que é um grau determinado com base nas propriedades dos adjetivos e do contexto de enunciação. A diferença crucial entre as duas propostas está no fato de que em Kennedy (2007) as informações do tipo de escala denotada pelo adjetivo também são computadas para a determinação desse grau, enquanto que, em Kennedy e McNally (2005), a variável C é uma variável livre contextual. Nesse sentido, vamos assumir a proposta de Kennedy e McNally (2005) de que a diferença entre pos e very está no parâmetro de comparação que é aumentado na entrada lexical de very, mas ela deve ser adaptada para a fórmula de pos de Kennedy (2007) que estamos adotando neste trabalho. Uma forma possível de fazer essa adpatação poderia ser por meio de uma mistura das duas propostas. Materíamos a denotação proposta para pos e incluiríamos uma conjunção com as informações da classe de comparação (238a). Mais especificamente, uma conjunção garantindo que a classe de comparação considerada na 
avaliação da sentença $O$ João é muito alto seja apenas a dos indivíduos altos (238b).

$$
\begin{aligned}
& \text { a. } \llbracket \text { pita } \rrbracket=\lambda \mathrm{G}_{<\mathrm{d},<\mathrm{e},>} \lambda \mathrm{x}_{\mathrm{e}} \cdot \exists \mathrm{d}\left[\mathrm{G}(\mathrm{d})(\mathrm{x}) \& \mathrm{~d} \geq \mathrm{d}_{\mathrm{z}} \& \operatorname{standard}\left(\mathrm{d}_{\mathrm{z}}\right)(\mathrm{C})\right. \\
& \text { b. } \llbracket \text { pita } \rrbracket=\lambda \mathrm{G}_{<\mathrm{d},<\mathrm{e}, \downarrow>} \lambda \mathrm{x}_{\mathrm{e}} \cdot \exists \mathrm{d}\left[\mathrm{G}(\mathrm{d})(\mathrm{x}) \& \mathrm{~d} \geq \mathrm{d}_{\mathrm{z}} \& \operatorname{standard}\left(\mathrm{d}_{\mathrm{z}}\right)(\lambda \mathrm{y} \cdot \llbracket \text { pos } \rrbracket(\mathrm{G})(\mathrm{y}))\right.
\end{aligned}
$$

A representação em (238b) garante que o grau preso existencialmente é maior que um grau $\mathrm{d}_{\mathrm{z}}$ avaliado de acordo com a classe de comparação dos indivíduos que já possuem o predicado G, como na proposta de Kennedyy e McNally (2005) para o very do inglês. No entanto, por questão de simplificação, vamos adotar nesta tese a proposta de Kennedy (2007) de que o grau $\mathbf{d}_{\mathbf{s}}$ representa a classe de comparação juntamente com as informações da escala do adjetivo e vamos adotar uma entrada lexical de pita sem a informação explícita da classe de comparação apresentada em (238). No entanto, vemos que (238) é facilmente derivada de pos. Logo, vamos assumir que o paramêtro de comparação é aumentado em $\mathbf{d}_{\text {s }}$ (Kennedy 2007).

O siginificado de pita com os adjetivos de escala fechada é um argumento a favor dessa escolha. Conforme apresentado acima, pita quando é usado para modificar predicados adjetivais de escalas fechadas, seleciona o grau máximo da escala denotada pelo adjetivo. Esse é exatamente o mesmo significado do morfema pos. Assim, semanticamente, não há diferença entre se enunciar $O$ cesto está cheio e $O$ cesto está completamente cheio. $\mathrm{O}$ fato de pita selecionar exatamente o grau que é representado por $\mathbf{d}_{\mathbf{s}}$ na proposta de Kennedy (2007) é um argumento a favor da escolha dessa proposta para o morfema pos em detrimento da proposta em Kennedy e McNally (2005), que não considera as propriedades dos adjetivos para a escolha do parâmetro de comparação. A proposta de Kennedy e McNally (2005) para o morfema pos com uma variável livre sobre classes de comparação deriva adequadamente o significado de adjetivos relativos em sua forma positiva. No entanto, a proposta dos autores não captura adequadamente a forma positiva de adjetivos absolutos porque não considera na formulação de pos uma informação sobre o tipo da escala do predicado graduável. Uma vez que a proposta de Kennedy (2007), com o grau $\mathbf{d}_{\mathbf{s}}$, consegue derivar as condições de verdade adequadas das sentenças com adjetivos relativos e absolutos, vamos continuar assumindo sua proposta.

Portanto, vamos seguir a proposta de Kennedy e McNally (2005) de que very e o morfema pos possuem o mesmo significado lexical, possuindo como diferença apenas os seus parâmetros contextuais e vamos propor para pita a mesma entrada lexical do morfema pos 
resguardadas as diferenças dos parâmetros contextuais, que na nossa proposta baseada em Kennedy (2007) são codificadas no grau $\mathbf{d}_{\mathbf{s}}$.

A entrada lexical de pita definitiva é, então, a seguinte:

$$
\llbracket \text { pita } \rrbracket=\lambda \mathrm{G}_{<\mathrm{d},<\mathrm{e}, \triangleright>>} \lambda \mathrm{x}_{\mathrm{e}} . \exists \mathrm{d}\left[\mathrm{G}(\mathrm{d})(\mathrm{x}) \& \mathrm{~d} \geq \mathrm{d}_{\mathrm{s}}\right]
$$

Em palavras, pita é uma função que pede um argumento graduável $\mathbf{G}$ de tipo $<\mathrm{d},<\mathrm{e}, \mathrm{t}>>$ (o adjetivo) e um argumento de indivíduos $\mathbf{x}$ de tipo e e devolve uma sentença de tipo t. Na sentença, o adjetivo $\mathbf{G}$ se aplica a $\mathbf{d}$ e a $\mathbf{x}$ e afirma que existe um grau $\mathbf{d}$ que é maior ou igual ao grau $\mathbf{d}_{\text {s. }} \mathrm{Na}$ fórmula em (239), $\mathbf{d}_{\mathbf{s}}$ representa o grau relevante em cada tipo de adjetivo. Assim como no caso da denotação do morfema pos proposta por Kennedy (2007), se o adjetivo tem escala aberta, o grau $\mathbf{d}_{\mathbf{s}}$ é representado pelo grau normal da escala, e a semântica do advérbio é de que o grau d é maior que o grau normal. No caso de pita, no entanto, $\mathbf{d}_{\mathbf{s}}$ será maior que o $\mathbf{d}_{\mathbf{s}}$ da forma positiva do adjetivo, derivando assim, a diferenteça entre é alto e é muito alto. Se o adjetivo for de escala fechada, o grau $\mathbf{d}_{\mathrm{s}}$ é representado pelo grau máximo da escala, e o significado do advérbio é de que o grau d é igual ao grau máximo da escala. O quadro abaixo resume a proposta.

\section{Tabela 42: Proposta para o Significado de Pita Modificando Adjetivos}

\begin{tabular}{|c|c|c|}
\hline \multicolumn{3}{|c|}{ Entrada Lexical } \\
\hline \multicolumn{3}{|c|}{$\llbracket$ pita $\rrbracket=\lambda \mathrm{G}_{<\mathrm{d},<\mathrm{e}, \triangleright>} \lambda \mathrm{x}_{\mathrm{e}} . \exists \mathrm{d}\left[\mathrm{G}(\mathrm{d})(\mathrm{x}) \& \mathrm{~d} \geq \mathrm{d}_{\mathrm{s}}\right]$} \\
\hline Tipo de Adjetivo & Valor de $\mathrm{d}_{\mathrm{s}}$ & Valor de d \\
\hline escala aberta & $\mathrm{d}_{\mathrm{s}}=$ grau normal & $\mathrm{d}>\mathrm{d}_{\mathrm{s}}$ \\
\hline escala fechada & $\mathrm{d}_{\mathrm{s}}=$ grau máximo & $\mathrm{d}=\mathrm{d}_{\mathrm{s}}$ \\
\hline
\end{tabular}

Essa semelhança no significado pode levar à hipótese de que pita é o próprio morfema pos e que finalmente foi encontrada uma língua em que ele fosse pronunciado. No entanto, uma análise nesse sentido perderia em poder de generalização quando se trata do uso do modificador no domínio verbal. Como se verá abaixo, quando pita(t) modifica predicados verbais atélicos ele tem um comportamente típico de um modificador de grau como a lot 'muito' do inglês e beacoup 'muito' do francês, resguardadas suas pequenas diferenças no significado. 
Dessa forma, vamos manter uma análise de que pita é um modificador de grau típico no sentido de que manipula valores em uma escala e tem distribuição em domínios sintáticos distintos. Abaixo, apresentamos dois exemplos de derivação de sentenças com adjetivos modificados por pita; primeiramente com o adjetivo de escala aberta $s e^{\prime} a$ 'bom' e em seguida com o adjetivo de escala fechada akyndop 'aberto'.

(240) Õwã se'a pita i-otam-Ø.

menino bom pita PART-chegar-ABS

'O menino muito bom chegou'

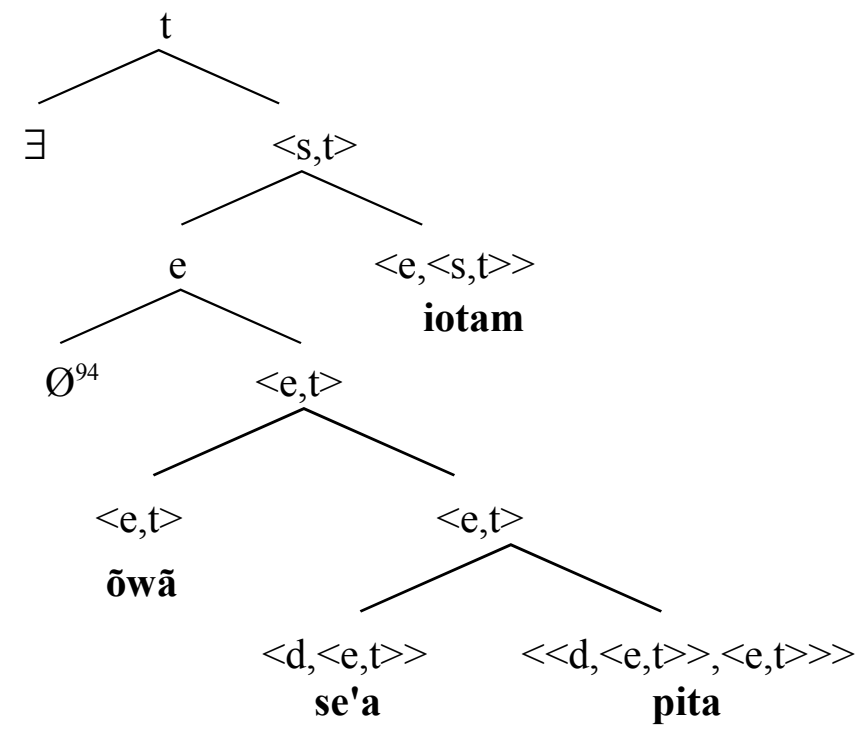

(242) Composição Semântica:

1. 【se'a pita $\rrbracket=\llbracket p i t a \rrbracket\left(\llbracket s e^{\prime} a \rrbracket\right) \quad$ Aplicação Funcional

$$
\begin{aligned}
& =\left[\lambda \mathrm{G} \lambda \mathrm{x} . \exists \mathrm{d}\left[\mathrm{G}(\mathrm{d})(\mathrm{x}) \& \mathrm{~d} \geq \mathrm{d}_{\mathrm{s}}\right]\right](\lambda \mathrm{d} \lambda \mathrm{x} . \text { "bondade" }(\mathrm{x})=\mathrm{d}) \\
& =\lambda \mathrm{x} . \exists \mathrm{d}\left[\text { "bondade" }(\mathrm{x})=\mathrm{d} \& \mathrm{~d}>\mathrm{d}_{\mathrm{s}}\right]
\end{aligned}
$$

2. $\llbracket o w \tilde{a} \rrbracket=\lambda \mathrm{x} \cdot \operatorname{menino}(\mathrm{x})$

Léxico

\footnotetext{
94 Uma vez que em Karitiana não há determinantes, estamos considerando a existência de um determinante nulo com o significado do artigo definido.
} 
3. 【 owã se'a pita $\rrbracket_{<\mathrm{e}, \downarrow}=\left[\lambda \mathrm{x} . \exists \mathrm{d}\left[\right.\right.$ "bondade" $\left.\left.(\mathrm{x})=\mathrm{d} \& \mathrm{~d}>\mathrm{d}_{\mathrm{s}}\right]\right][\lambda \mathrm{x} . \operatorname{menino}(\mathrm{x})]$

Regra de Modificação ${ }^{95}$

$$
=\lambda \mathrm{x} . \exists \mathrm{d}\left[\operatorname{menino}(\mathrm{x}) \& \text { "bondade" }(\mathrm{x})=\mathrm{d} \& \mathrm{~d}>\mathrm{d}_{\mathrm{s}}\right]
$$

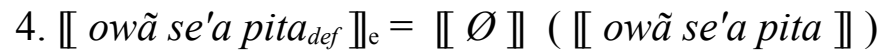

Aplicação Funcional

5. $\llbracket \varnothing \rrbracket=\lambda f$. o único $\mathrm{x}$ no contexto tal que $\mathrm{f}(\mathrm{x})=1$

Léxico

6. 【owã se'a pita ${ }_{\text {def }} \rrbracket_{\mathrm{e}}=[\lambda \mathrm{f}$. o único $\mathrm{x}$ no contexto tal que $\mathrm{f}(\mathrm{x})=1]$

$$
\begin{aligned}
& \left(\lambda \mathrm{x} . \exists \mathrm{d}\left[\operatorname{menino}(\mathrm{x}) \& \text { "bondade" }(\mathrm{x})=\mathrm{d} \& \mathrm{~d}>\mathrm{d}_{\mathrm{s}}\right]\right) \\
& =\mathrm{o} \text { único } \mathrm{x} \text { no contexto tal que }[\exists \mathrm{d}[\operatorname{menino}(\mathrm{x}) \& \\
& \text { "bondade" } \left.\left.(\mathrm{x})=\mathrm{d} \& \mathrm{~d}>\mathrm{d}_{\mathrm{s}}\right]\right]
\end{aligned}
$$

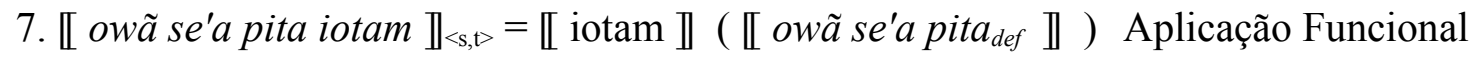

8. 【 iotam $\rrbracket=\lambda x \lambda$ e. chegar $(\mathrm{x})(\mathrm{e}) \quad$ Léxico

9. 【owã se'a pita iotam $\rrbracket_{<\mathrm{s}, \triangleright}=[\lambda \mathrm{x} \lambda \mathrm{e}$. chegar $(\mathrm{x})(\mathrm{e})]$ ( o único $\mathrm{x}$ no contexto tal que

$$
\left[\exists \mathrm{d}\left[\operatorname{menino}(\mathrm{x}) \& \text { "bondade" (x) }=\mathrm{d} \& \mathrm{~d}>\mathrm{d}_{\mathrm{s}}\right]\right] \text { ) }
$$

$=\lambda$ e. chegar (o único $x$ no contexto tal que $[\exists \mathrm{d}[$ menino(x)

$$
\text { \& "bondade" (x) } \left.=\mathrm{d} \& \mathrm{~d}>\mathrm{d}_{\mathrm{s}}\right] \text { ]) (e) }
$$

10. $\llbracket$ owã se'a pita iotam $\rrbracket_{\mathrm{t}}=1$ sse $\exists \mathrm{e} \exists \mathrm{d}$ [ chegar (o único $\mathrm{x}$ no contexto tal que menino(x)

$$
\left.\left.\& \text { "bondade" (x) }=\mathrm{d} \& \mathrm{~d}>\mathrm{d}_{\mathrm{s}}\right)(\mathrm{e})\right]
$$

Em palavras: A sentença Õwã se'a pita iotam é verdadeira se, e somente se, existe um evento e, e existe um grau d; o evento e é um evento de chegar do único $\mathbf{x}$ no contexto tal que $\mathbf{x}$ é menino e $\mathbf{x}$ é mapeado na escala de bondade em um grau $\mathbf{d}$ que é maior do que o grau $\mathbf{d}_{\mathbf{s}}$ (que representa o grau normal da escala, uma vez que se trata de uma escala aberta).

(243) Karamã akyndop pita i-pot-Ø.

porta aberto pita PART-quebrar-ABSГ

'A porta completamente aberta quebrou'

\footnotetext{
95 A Regra de Modificação é aplicada quando há dois predicados de mesmo tipo, como no caso acima de tentativa de composição semântica entre se'a pita 'muito bom' e õwã 'menino'. A Regra de Modificação estabelece que, sempre que houver dois predicados $\alpha$ e $\beta$ ambos de tipo $<\mathrm{e}, \mathrm{t}>$, eles podem formar juntos um novo predicado $\gamma$ que tem a seguinte entrada lexical:
}

(i) $\llbracket \gamma \rrbracket=\lambda \mathrm{x}$. $\alpha \rrbracket(\mathrm{x})=\llbracket \beta \rrbracket(\mathrm{x})=1$

(Heim e Kratzer 1998) 
(244)

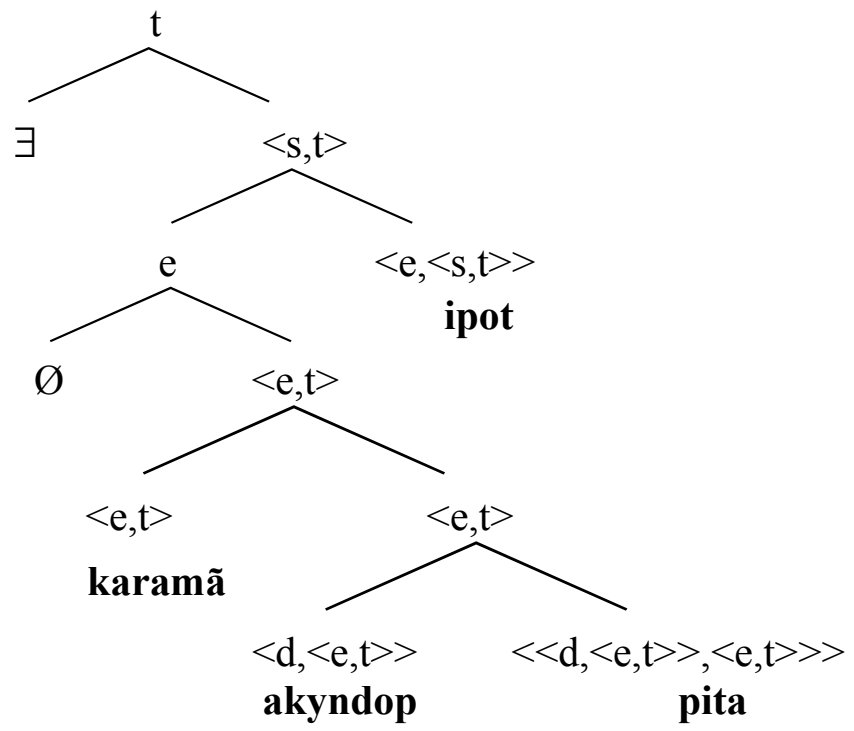

(245) Composição Semântica:

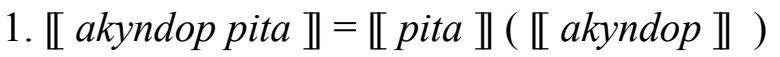

Aplicação Funcional

$=\left[\lambda \mathrm{G} \lambda \mathrm{x} . \exists \mathrm{d}\left[\mathrm{G}(\mathrm{d})(\mathrm{x}) \& \mathrm{~d} \geq \mathrm{d}_{\mathrm{s}}\right]\right](\lambda \mathrm{d} \lambda \mathrm{x}$. "abertura" $(\mathrm{x})=\mathrm{d})$

$=\lambda \mathrm{x} . \exists \mathrm{d}\left[\right.$ "abertura" (x) $\left.=\mathrm{d} \& \mathrm{~d}=\mathrm{d}_{\mathrm{s}}\right]$

2. $\llbracket \operatorname{karama} \tilde{a} \rrbracket=\lambda \mathrm{x} \cdot \operatorname{porta}(\mathrm{x})$

Léxico

3. 【 karamã akyndop pita $\rrbracket_{<\mathrm{e}, \mathrm{\downarrow}}=\left[\lambda \mathrm{x} . \exists \mathrm{d}\left[\right.\right.$ "abertura" $\left.\left.(\mathrm{x})=\mathrm{d} \& \mathrm{~d}=\mathrm{d}_{\mathrm{s}}\right]\right][\lambda \mathrm{x} \cdot \operatorname{porta}(\mathrm{x})]$

Regra de Modificação

$$
=\lambda \mathrm{x} . \exists \mathrm{d}\left[\operatorname{porta}(\mathrm{x}) \& \text { "abertura" }(\mathrm{x})=\mathrm{d} \& \mathrm{~d}=\mathrm{d}_{\mathrm{s}}\right]
$$

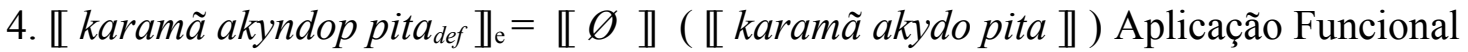

5. 【Ø $\rrbracket=\lambda f$. o único $x$ no contexto tal que $\mathrm{f}(\mathrm{x})=1$

Léxico

6. 【 karamã akyndop pita def $\rrbracket=[\lambda \mathrm{f}$. o único $\mathrm{x}$ no contexto tal que $\mathrm{f}(\mathrm{x})=1$ ]

$$
\left(\lambda x . \exists d\left[\operatorname{porta}(x) \& \text { "abertura" }(x)=d \& d=d_{s}\right]\right)
$$

$=\mathrm{o}$ único $\mathrm{x}$ no contexto tal que $[\exists \mathrm{d}[\operatorname{porta}(\mathrm{x}) \&$ "abertura" $(\mathrm{x})=\mathrm{d} \& \mathrm{~d}=\mathrm{d}_{\mathrm{s}}$ ] ]

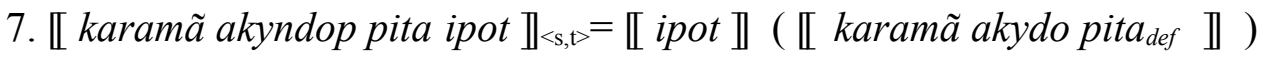

Aplicação Funcional

8. 【 ipot $\rrbracket=\lambda \mathrm{x} \lambda$ e. quebrar $(\mathrm{x})(\mathrm{e})$ 
9. 【 karamã akyndop pita ipot $\rrbracket_{<, t \triangleright}=[\lambda \mathrm{x} \lambda \mathrm{e}$. quebrar $(\mathrm{x})(\mathrm{e})]$ ( o único $\mathrm{x}$ no contexto tal

$$
\text { que } \left.\left[\exists \mathrm{d}\left[\operatorname{porta}(\mathrm{x}) \& \text { “abertura” }(\mathrm{x})=\mathrm{d} \& \mathrm{~d}=\mathrm{d}_{\mathrm{s}}\right]\right]\right)
$$

$=\lambda$ e. quebrar (o único $x$ no contexto tal que $[\exists \mathrm{d}$

$\left[\operatorname{porta}(\mathrm{x}) \&\right.$ "abertura" (x) $\left.\left.\left.=\mathrm{d} \& \mathrm{~d}=\mathrm{d}_{\mathrm{s}}\right]\right]\right)(\mathrm{e})$

10. 【 karamã akyndop pita ipot $\rrbracket_{\mathrm{t}}=1 \mathrm{sse} \exists \mathrm{e} \exists \mathrm{d}$ [ quebrar (o único x no contexto tal que

$$
\left.\operatorname{porta}(\mathrm{x}) \& \text { “abertura" }(\mathrm{x})=\mathrm{d} \& \mathrm{~d}=\mathrm{d}_{\mathrm{s}}\right)(\mathrm{e})
$$

Em palavras: A sentença Karamã akydo pita ipot é verdadeira se, e somente se, existe um evento e, e existe um grau $\mathbf{d}$; o evento e é um evento de quebrar do único $\mathbf{x}$ no contexto tal que $\mathbf{x}$ é porta e $\mathbf{x}$ é mapeado na escala de abertura em um grau $\mathbf{d}$ que é igual ao grau $\mathbf{d}_{\mathbf{s}}$ (que representa o grau máximo da escala, uma vez que se trata de uma escala fechada).

Esta seção mostrou que o modificador de grau pita quando é aplicado a adjetivos não seleciona exclusivamente nenhum tipo de escala, diferentemente do que ocorre com os modificadores de grau do inglês very, much e well. No entanto, a interpretação de pita varia de acordo com o predicado modificado. Se o predicado adjetival é de escala aberta seu significado é algo como 'acima de um grau normal na escala', semântica usualmente atribuída ao modificador muito. Se o predicado é de escala fechada, sua interpretação é 'no grau máximo da escala', significado de completamente. A seção mostrou que, a despeito dessa variação, é possível oferecer uma análise unificada para as sentenças contendo adjetivos modificados por pita por meio de uma entrada lexical única para o advérbio baseada no grau $\mathrm{d}_{\mathrm{s}}$ que corresponde ao ponto de corte da escala. 


\subsection{Verbos}

Esta seção pretende apresentar a distribuição do advérbio pitat em Karitiana com sintagmas verbais. Os dados da língua mostram que pitat tem um significado canônico de modificador de grau somente quando modifica predicados verbais atélicos. Quando modifica predicados télicos, pitat tem um significado parecido com o de uma confirmação, algo como mesmo. O significado de pitat será analisado em detalhes mais abaixo. Vamos, primeiramente, analisar as possibilidades de adjunção de pitat e sua variante fonológica pitadn. A tabela abaixo apresenta a tabela de verbos apresentada no capítulo 3 com verbos modificados por pitat.

Tabela 43: Predicados Verbais Modificados por Pitat

\begin{tabular}{|c|c|}
\hline Verbo & Exemplo \\
\hline \multicolumn{2}{|r|}{ Atividades } \\
\hline $\begin{array}{l}\text { pykyn } \\
\text { 'correr' }\end{array}$ & $\begin{array}{l}\text { João i-pykyn-<a>-t pita-t. } \\
\text { João PART-correr-ABS pita-ADV } \\
\text { 'O João correu muito' }\end{array}$ \\
\hline $\begin{array}{l}\text { pytim'adn } \\
\text { 'trabalhar' }\end{array}$ & $\begin{array}{l}\text { João i-pytim'adn- }<\mathrm{a}>-\mathrm{t} \text { pita-t. } \\
\text { João PART-trabalhar-ABS pita-ADV } \\
\text { 'O João trabalhou muito' }\end{array}$ \\
\hline $\begin{array}{l}\text { pimbik gooj } \\
\text { 'empurrar carro' }\end{array}$ & $\begin{array}{l}\text { João Ø-na-pimbik-Ø } \quad \text { pita-t } \quad \text { gooj. } \\
\text { João 3-DECL-empurrar-NFUT } \\
\text { 'O Jita-ADV carro } \\
\text { 'O João empurrou muito o carro' }\end{array}$ \\
\hline $\begin{array}{l}\text { tagngã gooj } \\
\text { 'dirigir carro' }\end{array}$ & $\begin{array}{l}\text { João Ø-naka-tagngã-t } \quad \text { pita-t } \quad \text { gooj. } \\
\text { João 3-DECL-dirigir-NFUT pita-ADV carro } \\
\text { 'O João dirigiu muito o carro' }\end{array}$ \\
\hline \multicolumn{2}{|r|}{ Estativos Stage-level } \\
\hline $\begin{array}{l}\text { siki'y } \\
\text { 'querer' }\end{array}$ & $\begin{array}{l}\text { João i-siki'y-t pita-t } \quad \text { ese-ty. } \\
\text { João PART-querer-ABS pita-ADV água-OBL } \\
\text { 'O João quis muito água' }\end{array}$ \\
\hline
\end{tabular}




\begin{tabular}{|c|c|}
\hline $\begin{array}{l}\text { osedn } \\
\text { 'estar alegre' }\end{array}$ & $\begin{array}{l}\text { João i-osedn- } \varnothing \text { pita-t. } \\
\text { João PART-estar.alegre-ABS pita-ADV } \\
\text { 'O João ficou muito alegre' }\end{array}$ \\
\hline $\begin{array}{l}\text { em } \\
\text { 'estar sujo' }\end{array}$ & $\begin{array}{l}\text { João i-em- } \varnothing \quad \text { pita-t. } \\
\text { João PART-estar.sujo-ABS pita-ADV } \\
\text { 'O João ficou muito sujo' } \\
\end{array}$ \\
\hline $\begin{array}{l}\text { pasadn } \\
\text { 'gostar' }\end{array}$ & $\begin{array}{l}\text { João i-pasadn- } \varnothing \quad \text { pita-t } \quad \text { Milena-ty. } \\
\text { João PART-gostar-ABS } \text { pita-ADV Milena-OBL } \\
\text { 'O João gostou muito da Milena' }\end{array}$ \\
\hline & Estativos Individual-level \\
\hline $\begin{array}{l}\text { aka Karitiana } \\
\text { 'ser Karitiana' }\end{array}$ & $\begin{array}{lll}\text { *João Ø-na-aka-t } & \text { pita-t } & \text { Karitiana-t. } \\
\text { João 3-DECL-COP-NFUT } & \text { pita-ADV } & \text { Karitiana-ABS } \\
\text { Tradução pretendida: 'O João é Karitiana mesmo' }\end{array}$ \\
\hline $\begin{array}{l}\text { pypyyt Matematicaty } \\
\text { 'saber Matemática' }\end{array}$ & $\begin{array}{l}\text { João i-pypyyt- } \varnothing \quad \text { pita-t } \quad \text { Matematica-ty. } \\
\text { João PART-saber-ABS } \\
\text { 'O João aprendeu mesmo Matemática' } \\
\end{array}$ \\
\hline $\begin{array}{l}\text { kywytidn Botỹjty } \\
\text { 'acreditar em Deus' }\end{array}$ & $\begin{array}{l}\text { João i-kywytidn-<a>-t pita-t Botỹj-ty. } \\
\text { João PART-acreditar-ABS pita-ADV Deus-OBL } \\
\text { 'O João acredita muito em em Deus' }\end{array}$ \\
\hline & Accomplishment \\
\hline $\begin{array}{l}\text { kokot Sete de } \\
\text { Setembro dewota } \\
\text { kyyn } \\
\text { 'atravessar a Sete de } \\
\text { Setembro' }\end{array}$ & $\begin{array}{l}\text { João i-kokot- } \varnothing \quad \text { pita-t Sete de Setembro } \\
\text { João PART-passar-ABS pita-ADV Sete de Setembro } \\
\text { dewota kyyn. } \\
\text { outro.lado para } \\
\text { 'O João atravessou mesmo a rua Sete de Setembro' }\end{array}$ \\
\hline $\begin{array}{l}\text { pog pyejepa goot } \\
\text { 'pintar a escola nova } \\
\text { de branco' }\end{array}$ & $\begin{array}{l}\text { João } Ø \text {-naka-pog-<a>-t pita-t } \quad \text { pyejepa goot. } \\
\text { João 3-DECL-pintar.de.branco-NFUT pita-ADV escola novo } \\
\text { 'O João pintou mesmo a escola nova de branco' }\end{array}$ \\
\hline $\begin{array}{l}\text { tat Porto Velho pip } \\
\text { 'ir para Porto Velho' }\end{array}$ & $\begin{array}{l}\text { João itat } \quad \text { pita-t } \quad \text { Porto Velho pip. } \\
\text { João PART-ir-ABS } \text { pita-ADV Porto Velho para } \\
\text { 'O João foi mesmo para Porto Velho' }\end{array}$ \\
\hline
\end{tabular}




\begin{tabular}{|c|c|}
\hline $\begin{array}{l}\text { 'a myhint boet } \\
\text { 'fazer um colar' }\end{array}$ & $\begin{array}{l}\text { João Ø-naka-m-'a-t } \quad \text { pita-t } \quad \text { myhin-t boet. } \\
\text { João 3-DECL- CAUS-fazer-NFUT pita-ADV um-OBL colar } \\
\text { 'O João fez mesmo um colar' }\end{array}$ \\
\hline \multicolumn{2}{|r|}{ Achievement } \\
\hline $\begin{array}{l}\text { pop } \\
\text { 'morrer' }\end{array}$ & $\begin{array}{l}\text { João i-pop-Ø pita-t. } \\
\text { João PART-morrer-ABS pita-ADV } \\
\text { 'O João morreu mesmo' }\end{array}$ \\
\hline $\begin{array}{l}\text { otam } \\
\text { 'chegar' }\end{array}$ & $\begin{array}{l}\text { João i-otam- } \varnothing \quad \text { pita-t. } \\
\text { João PART-chegar-ABS pita-ADV } \\
\text { 'O João chegou mesmo' }\end{array}$ \\
\hline $\begin{array}{l}\text { typ Antônio Bigode } \\
\text { 'encontrar Antônio } \\
\text { Bigode' }\end{array}$ & $\begin{array}{l}\text { João Ø-naka-typ-Ø } \quad \text { pita-t Antônio Bigode. } \\
\text { João 3-DECL-encontrar-NFUT pita-ADV Antônio Bigode } \\
\text { 'O João encontrou mesmo o Antônio Bigode' }\end{array}$ \\
\hline $\begin{array}{l}\text { otam myhint deso } \\
\text { ohyn sok } \\
\text { 'chegar no topo de } \\
\text { uma montanha' }\end{array}$ & $\begin{array}{l}\text { João i-otam- } \varnothing \text { pita-t myhin-t deso } \text { ohyn sok. } \\
\text { João PART-chegar-ABS pita-ADV um-OBL montanha topo em } \\
\text { 'O João chegou mesmo no topo de uma montanha' }\end{array}$ \\
\hline
\end{tabular}

A tabela acima mostra que pitat pode ser usado com todos os tipos de predicados verbais, com exceção de aka Karitiana 'ser Karitiana' que é o predicado individual-level por excelência. Conforme o capítulo anterior mostrou, verbos individual-level têm uma resistência de serem modificados. Para Kratzer (1995), isso é uma evidência de que esses predicados não tem um argumento de evento. O predicado pypyyt Matematica, que pode significar 'saber Matemática' (estativo individual-level) ou 'aprender Matemática' (verbo de achievement), foi traduzido como a segunda opção. A explicação pode ser exatamente a presença do advérbio que não pode modificar um predicado sem argumento evento e forçaria a mudança na interpretação do predicado. Já o VP kywytidn Botỹjty 'acreditar em Deus' passa a ser interpretado como um predicado stage-level com a presença do advérbio. As possibilidade de situações em que a se pode usar a sentença modificada que serão discutidas a seguir confirmam essa mudança no tipo de predicado.

A tabela mostrou ainda que pitat tem um comportamento previsível com os 
predicados verbais. Quando aparece com os sintagmas verbais atélicos, ele tem um significado parecido com o de muito em português. Quando modifica predicados télicos, pitat tem um significado de confirmação e pode ser traduzido por 'mesmo'. A tabela foi utilizada apenas para uma primeira apresentação da possibilidade de uso do advérbio pitat com os verbos selecionados. O significado de pitat em cada contexto será discutido com detalhes a seguir.

Antes de analisar o significado de pitat em cada contexto verbal, vamos apresentar ainda duas características da distribuição do advérbio na língua, sua posição de adjunção e sua variante pitadn. A primeira observação que vamos fazer dos dados acima é que, diferentemente de outros advérbios da língua, pitat pode ocorrer somente na posição pós verbal. Conforme apresentado no capítulo 1, Storto (1999) analisou a adjunção adverbial em Karitiana e notou que, em sentenças matrizes, a única posição que um advérbio não pode ocupar é entre o sujeito e o verbo, porque, segundo a análise da autora, esses dois constituintes estão em uma configuração especificador-núcleo (do inglês spec-head). Os dados abaixo ilustram esse fato com a distribuição do advérbio mynda 'vagarosamente' em sentenças transitivas:

a. Mynda

taso Ø-na-m-potpora-j

ese. $=(4$ e 5$)$

vagarosamente homem 3-DECL-CAUS-ferver-FUTágua

'O homem ferveu a água vagarosamente'

b. Taso Ø-na-m-potpora-j mynda ese.

homem 3-DECL-CAUS-ferver-FUTvagarosamente água

'O homem ferveu a água vagarosamente'

$\begin{array}{llll}\text { c. } & \text { Taso } \quad \text { Ø-na-m-potpora-j } & \text { ese } & \text { mynda. } \\ \text { homem } & \text { 3-DECL-CAUS-ferver-FUT } & \text { água } & \text { vagarosamente } \\ \text { 'O homem ferveu a água vagarosamente' } & \end{array}$


d. * Taso mynda Ø-na-m-potpora-j ese.

homem vagarosamente 3-DECL-CAUS-ferver-FUTágua

'O homem ferveu a água vagarosamente'

(exemplos 58 a 61 de Storto 1999, p. 138 e 139)

O quadro abaixo mostra que pitat não tem a mesma distribuição que os outros advérbios da língua. Ele não pode ocorrer em nenhuma outra posição que não seja depois do verbo.

Tabela 44: (Im)possibilidades de Adjunção de Pitat

\begin{tabular}{|c|c|}
\hline Verbo & Exemplo \\
\hline \multicolumn{2}{|r|}{ Atividades } \\
\hline $\begin{array}{l}\text { pykyn } \\
\text { 'correr' }\end{array}$ & $\begin{array}{l}\text { *Pita-t João i-pykyn-<a>-t. } \\
\text { pita-ADV João PART-correr-ABS } \\
\text { Tradução pretendida: 'O João correu muito' } \\
\text { *João pita-t i-pykyn-<a>-t. } \\
\text { João pita-ADV PART-correr-ABS } \\
\text { Tradução pretendida: 'O João correu muito' } \\
\text { *Pita-t i-pykyn-<a>-t } \\
\text { pita-ADV PART-CORRER-ABS João } \\
\text { Tradução pretendida: 'O João correu muito' }\end{array}$ \\
\hline $\begin{array}{l}\text { pytim'adn } \\
\text { 'trabalhar' }\end{array}$ & $\begin{array}{l}\text { * Pita-t João i-pytim'adn-<a>-t. } \\
\text { pita-ADV João PART-trabalhar-ABS } \\
\text { Tradução pretendida: 'O João trabalhou muito' } \\
\text { *João pita-t i-pytim'adn-<a>-t. } \\
\text { João pita-ADV PART-trabalhar-ABS } \\
\text { Tradução pretendida: 'O João trabalhou muito' }\end{array}$ \\
\hline
\end{tabular}




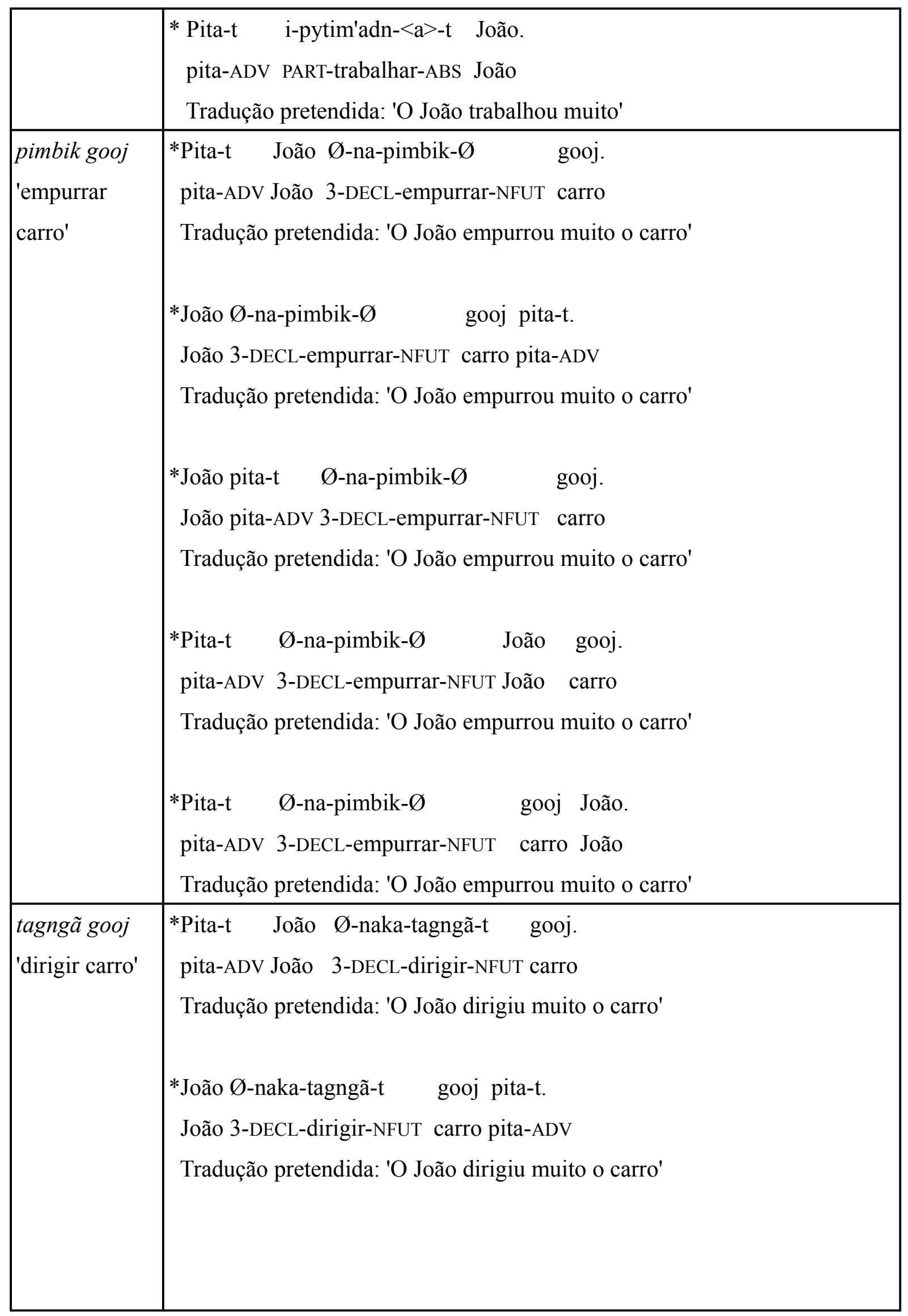




\begin{tabular}{|c|c|}
\hline & $\begin{array}{l}\text { *João pita-t } \quad \text { Ø-naka-tagngã-t } \quad \text { gooj. } \\
\text { João pita-ADV 3-DECL-dirigir-NFUT carro } \\
\text { Tradução pretendida: 'O João dirigiu muito o carro' } \\
\text { *Pita-t } \quad \text { Ø-naka-tagngã-t } \quad \text { João gooj. } \\
\text { pita-ADV 3-DECL-dirigir-NFUT João carro } \\
\text { Tradução pretendida: 'O João dirigiu muito o carro' } \\
\text { *Pita-t } \quad \text { Ø-naka-tagngã-t } \\
\text { pita-ADV 3-DECL-dirigir-NFUT carro João } \\
\text { Tradução pretendida: 'O João dirigiu muito o carro' }\end{array}$ \\
\hline & Estativos Stage-level \\
\hline $\begin{array}{l}\text { siki'y } \\
\text { 'querer' }\end{array}$ & $\begin{array}{l}\text { *Pita-t João i-siki'y-t ese-ty. } \\
\text { pita-ADV João PART-querer-ABS água-OBL } \\
\text { Tradução pretendida: 'O João quis muito água' } \\
\text { *João i-siki'y-t ese-ty pita-t. } \\
\text { João PART-querer-ABS água-OBL pita-ADV } \\
\text { Tradução pretendida: 'O João quis muito água' } \\
\text { *João pita-t i-siki'y-t } \\
\text { João pita-ADV PART-querer-ABS água-OBL } \\
\text { Tradução pretendida: 'O João quis muito água' } \\
\text { *Pita-t i-siki'y-t } \\
\text { pita-ADV PART-querer-ABS João água-OBL } \\
\text { Tradução pretendida: 'O João quis muito água' } \\
\text { *Pita-t i-siki'y-t } \\
\text { pradução pretendida: 'O João quis muito água' }\end{array}$ \\
\hline
\end{tabular}




\begin{tabular}{|c|c|}
\hline $\begin{array}{l}\text { osedn } \\
\text { 'estar alegre' }\end{array}$ & $\begin{array}{l}\text { *Pita-t João i-osedn- } \varnothing \text {. } \\
\text { pita-ADV João PART-estar.alegre-ABS } \\
\text { Tradução pretendida: 'O João ficou muito alegre' } \\
\text { *João pita-t i-osedn- } \varnothing . \\
\text { João pita-ADV PART-estar.alegre-ABS } \\
\text { Tradução pretendida: 'O João ficou muito alegre' } \\
\text { *Pita-t i-osedn- } \varnothing \\
\text { pita-ADV PART-estar.alegre-ABS João } \\
\text { Tradução pretendida: 'O João ficou muito alegre' }\end{array}$ \\
\hline $\begin{array}{l}\text { em } \\
\text { 'estar sujo' }\end{array}$ & $\begin{array}{l}\text { *Pita-t João i-em-Ø. } \\
\text { pita-ADV João PART-estar.sujo-ABS } \\
\text { Tradução pretendida: 'O João ficou muito sujo' } \\
\text { *João pita-t i-em-Ø. } \\
\text { João pita-ADV PART-estar.sujo-ABS } \\
\text { Tradução pretendida: 'O João ficou muito sujo' } \\
\text { *Pita-t i-em-Ø } \\
\text { pita-ADV PART-estar.sujo-ABS João } \\
\text { Tradução pretendida: 'O João ficou muito sujo' }\end{array}$ \\
\hline $\begin{array}{l}\text { pasadn } \\
\text { 'gostar' }\end{array}$ & $\begin{array}{l}\text { *Pita-t João i-pasadn- } \varnothing \quad \text { Milena-ty. } \\
\text { pita-ADV João PART-gostar-ABS Milena-OBL } \\
\text { Tradução pretendida: 'O João gostou muito da Milena' } \\
\text { *João i-pasadn- } \varnothing \quad \text { Milena-ty pita-t. } \\
\text { João PART-gostar-ABS Milena-OBL pita-ADV } \\
\text { Tradução pretendida: 'O João gostou muito da Milena' }\end{array}$ \\
\hline
\end{tabular}




\begin{tabular}{|c|c|}
\hline & $\begin{array}{l}\text { *João pita-t i-pasadn- } \varnothing \quad \text { Milena-ty. } \\
\text { João pita-ADV PART-gostar-ABS Milena-OBL } \\
\text { Tradução pretendida: 'O João gostou muito da Milena' } \\
\text { *Pita-t i-pasadn- } \varnothing \quad \text { João Milena-ty. } \\
\text { pita-ADV PART-gostar-ABS João Milena-OBL } \\
\text { Tradução pretendida: 'O João gostou muito da Milena' } \\
\text { *Pita-t i-pasadn- } \varnothing \quad \text { Milena-ty João. } \\
\text { pita-ADV PART-gostar-ABS Milena-OBL João } \\
\text { Tradução pretendida: 'O João gostou muito da Milena' }\end{array}$ \\
\hline & Estativos Individual-level \\
\hline $\begin{array}{l}\text { pypyyt } \\
\text { Matematicaty } \\
\text { 'saber } \\
\text { Matemática' }\end{array}$ & 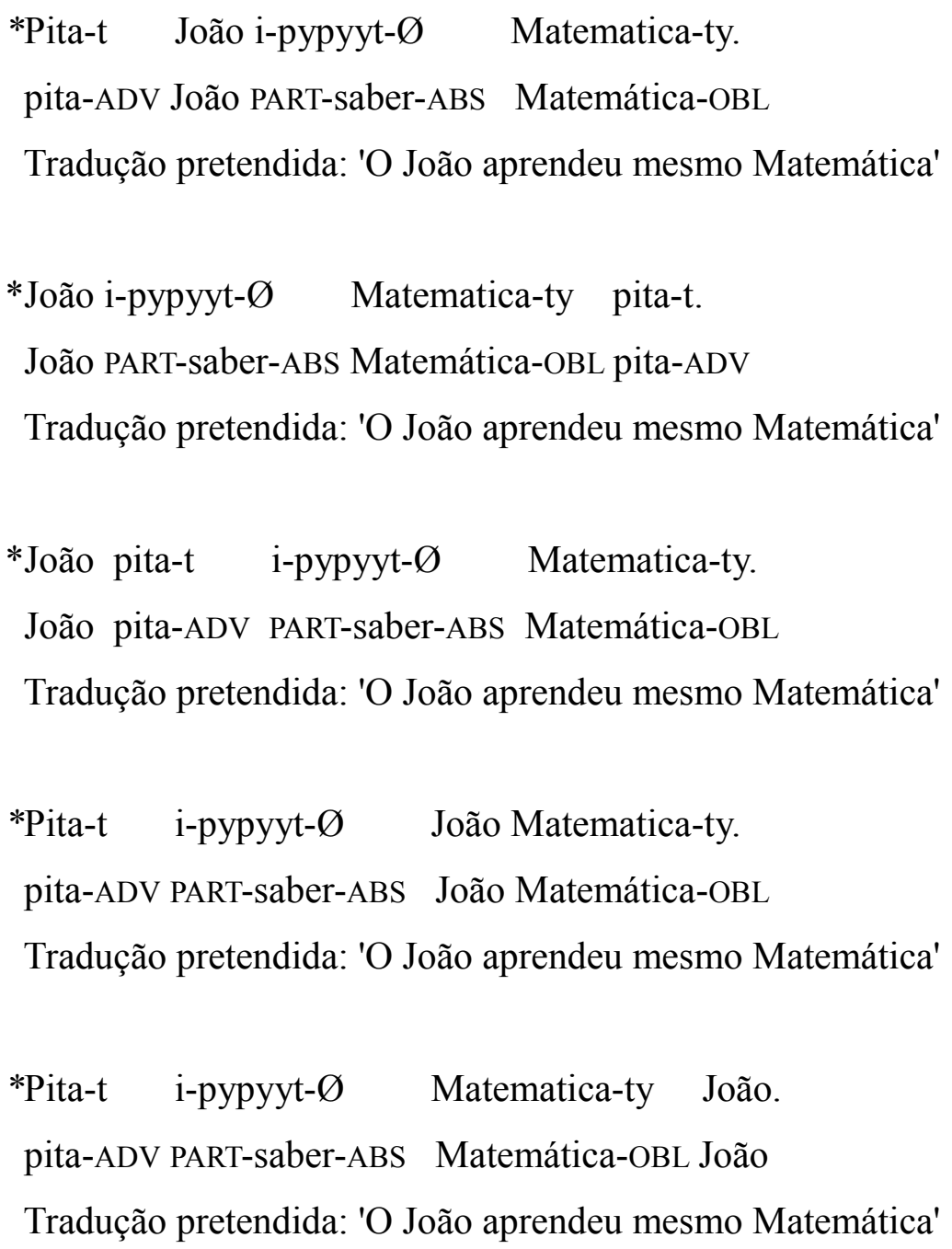 \\
\hline
\end{tabular}




\begin{tabular}{|c|c|}
\hline $\begin{array}{l}\text { kywytidn } \\
\text { Botỹjty } \\
\text { 'acreditar em } \\
\text { Deus' }\end{array}$ & $\begin{array}{l}\text { *Pita-t João i-kywytidn-<a>-t Botỹj-ty. } \\
\text { pita-ADV João PART-acreditar-ABS Deus-OBL } \\
\text { Tradução pretendida: 'O João acredita muito em em Deus' } \\
\text { *João i-kywytidn-<a>-t Botỹj-ty pita-t. } \\
\text { João PART-acreditar-ABS Deus-OBL pita-ADV } \\
\text { Tradução pretendida: 'O João acredita muito em em Deus' } \\
\text { *João pita-t } \\
\text { João pita-ADV PART-acreditar-ABS Deus-OBL } \\
\text { Tradução pretendida: 'O João acredita muito em em Deus' } \\
\text { *Pita-t } \quad \text { i-kywytidn-<a>-t João Botỹj-ty. } \\
\text { pita-ADV PART-acreditar-ABS João Deus-OBL } \\
\text { Tradução pretendida: 'O João acredita muito em em Deus' } \\
\text { pita-ADV PART-acreditar-ABS Deus-OBL João } \\
\text { Tradução pretendida: 'O João acredita muito em em Deus' } \\
\text { i-kywytidn-<a>-t Botỹj-ty João. }\end{array}$ \\
\hline & Accomplishment \\
\hline $\begin{array}{l}\text { kokot Sete de } \\
\text { Setembro } \\
\text { dewota kyyn } \\
\text { 'atravessar a } \\
\text { Sete de } \\
\text { Setembro' }\end{array}$ & 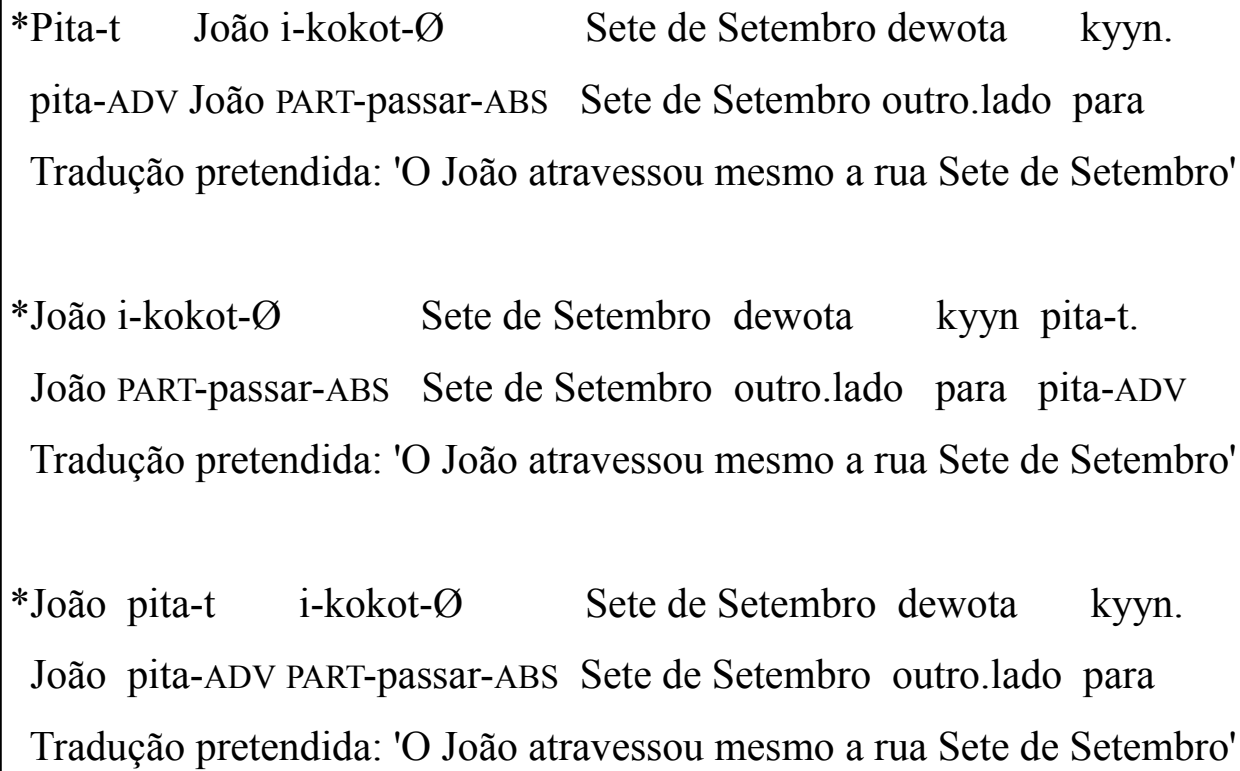 \\
\hline
\end{tabular}




\begin{tabular}{|c|c|}
\hline & 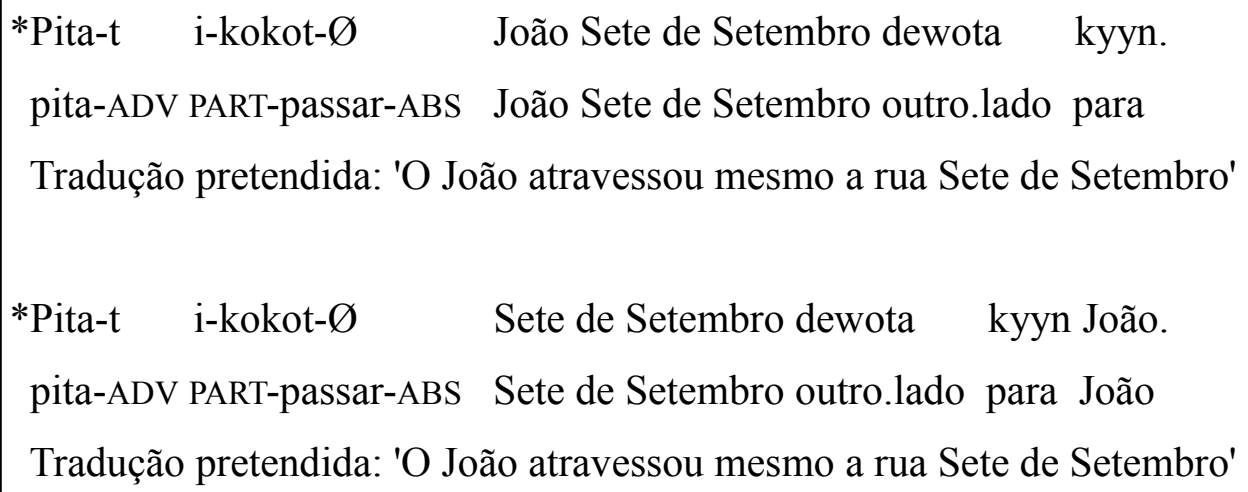 \\
\hline $\begin{array}{l}\text { pog pyejepa } \\
\text { goot } \\
\text { 'pintar a escola } \\
\text { nova de } \\
\text { branco' }\end{array}$ & 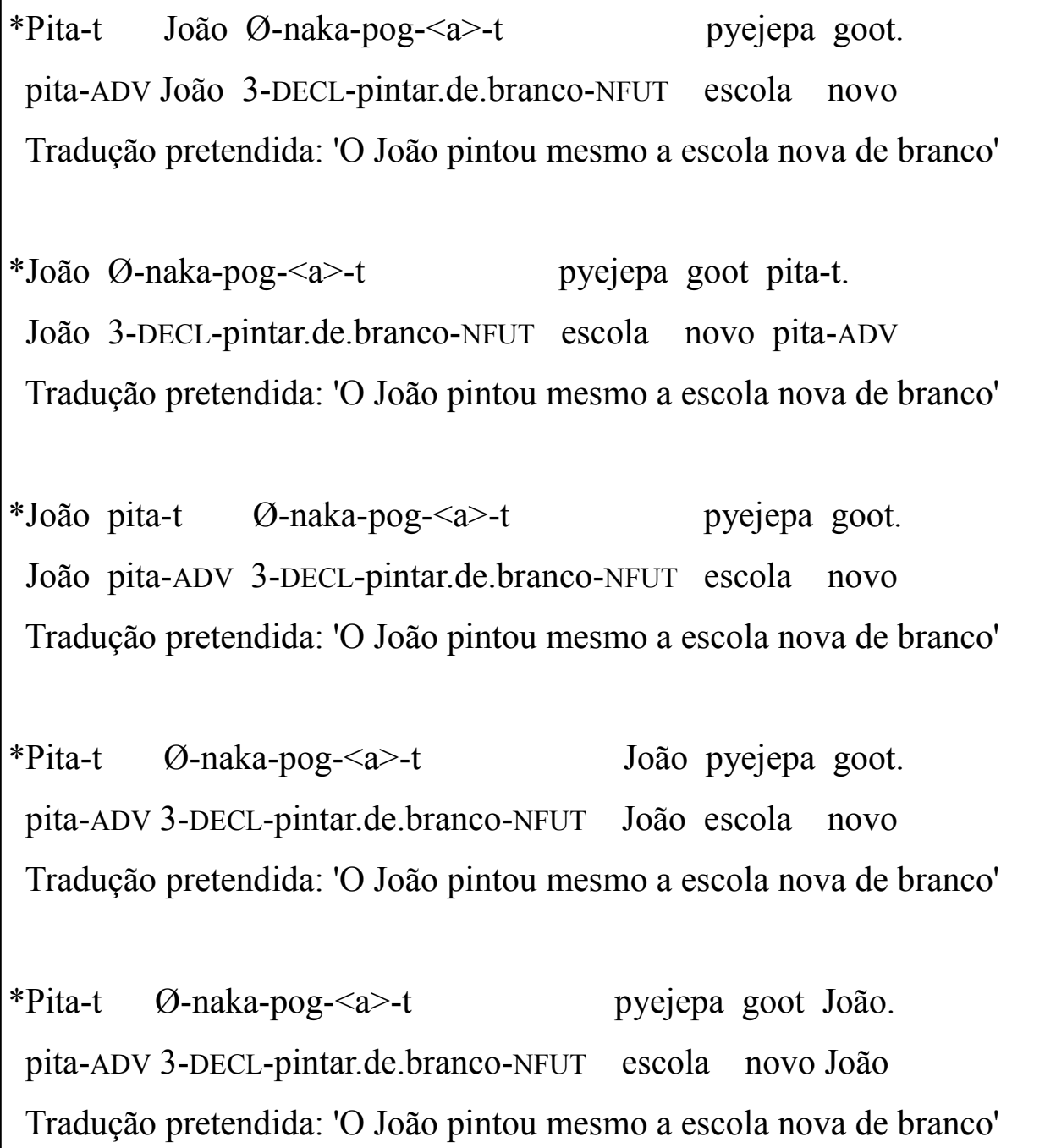 \\
\hline $\begin{array}{l}\text { tat Porto } \\
\text { Velho pip } \\
\text { 'ir para Porto } \\
\text { Velho' }\end{array}$ & $\begin{array}{l}\text { *Pita-t João i-tat- } \varnothing \quad \text { Porto Velho pip. } \\
\text { pita-ADV João } \\
\text { PART-ir-ABS Porto Velho para } \\
\text { Tradução pretendida: 'O João foi mesmo para Porto Velho' }\end{array}$ \\
\hline
\end{tabular}




\begin{tabular}{|c|c|}
\hline & 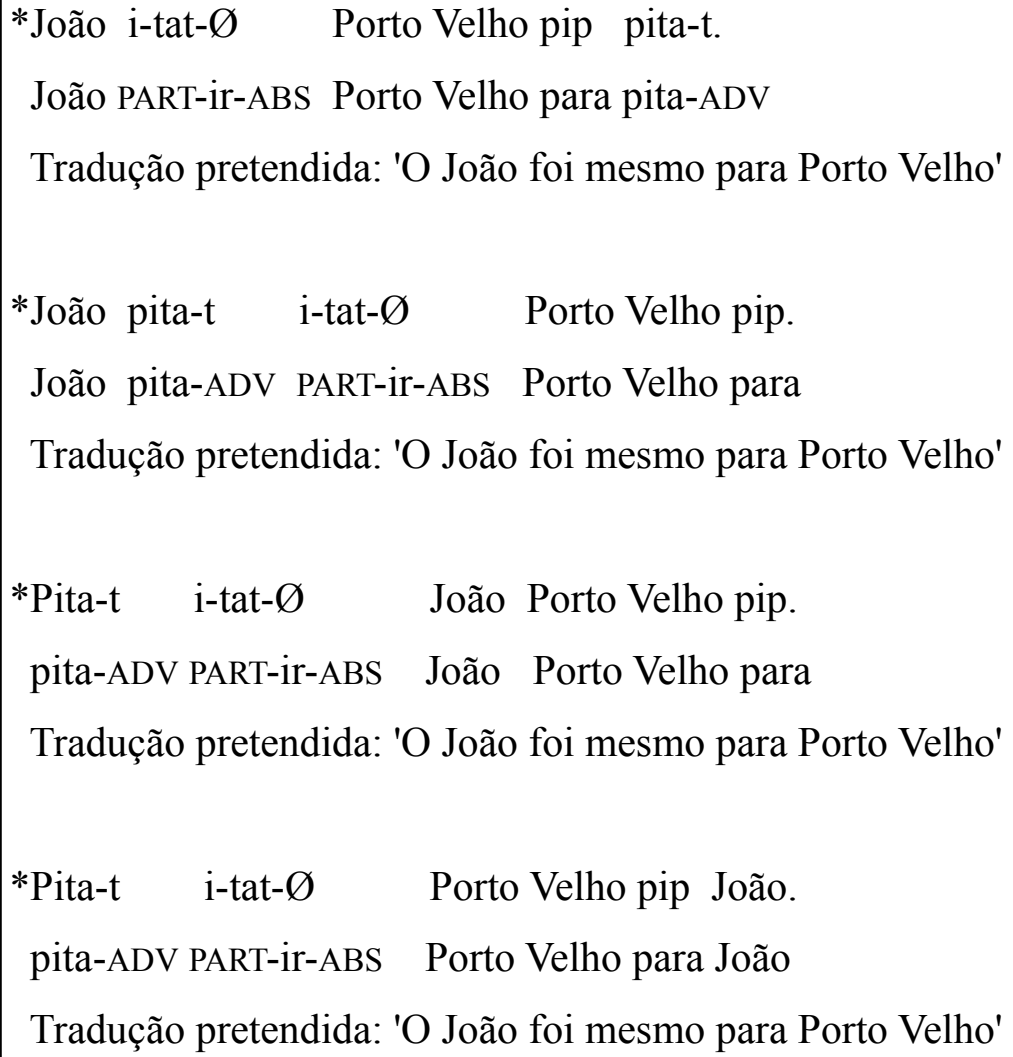 \\
\hline $\begin{array}{l}\text { 'a myhint boet } \\
\text { 'fazer um } \\
\text { colar' }\end{array}$ & $\begin{array}{l}\text { *Pita-t João Ø-naka-m-'a-t myhin-t boet. } \\
\text { pita-ADV João 3-DECL-CAUS-fazer-NFUT um-OBL colar } \\
\text { Tradução pretendida: 'O João fez mesmo um colar' } \\
\text { *João Ø-naka-m-'a-t myhin-t boet pita-t. } \\
\text { João 3-DECL-CAUS-fazer-NFUT um-OBL colar pita-ADV } \\
\text { Tradução pretendida: 'O João fez mesmo um colar' } \\
\text { *João pita-t } \quad \text { Ø-naka-m-'a-t } \\
\text { João pita-ADV 3-DECL-CAUS-fazer-NFUT um-OBL colar } \\
\text { Tradução pretendida: 'O João fez mesmo um colar' } \\
\text { *Pita-t } \quad \text { m-naka-m-'a-t } \\
\text { pita-ADV 3-DECL-CAUS-fazer-NFUT João um-OBL colar } \\
\text { Tradução pretendida: 'O João fez mesmo um colar' }\end{array}$ \\
\hline
\end{tabular}




\begin{tabular}{|c|c|}
\hline & $\begin{array}{llll}\text { *Pita-t } & \varnothing \text {-naka-m-'a-t } & \text { myhin-t boet } & \text { João. } \\
\text { pita-ADV } & \text { 3-DECL-CAUS-fazer-NFUT } & \text { um-OBL colar } & \text { João } \\
\text { Tradução pretendida: 'O João fez mesmo um colar' }\end{array}$ \\
\hline \multicolumn{2}{|r|}{ Achievement } \\
\hline $\begin{array}{l}\text { рор } \\
\text { 'morrer' }\end{array}$ & $\begin{array}{l}\text { *Pita-t João i-pop- } \varnothing . \\
\text { pita-ADV João PART-morrer-ABS } \\
\text { Tradução pretendida: 'O João morreu mesmo' } \\
\text { *João pita-t i-pop-Ø. } \\
\text { João pita-ADV PART-morrer-ABS } \\
\text { Tradução pretendida: 'O João morreu mesmo' } \\
\text { *Pita-t i-pop- } ~ \\
\text { pita-ADV PART-morrer-ABS João } \\
\text { Tradução pretendida: 'O João morreu mesmo' }\end{array}$ \\
\hline $\begin{array}{l}\text { otam } \\
\text { 'chegar' }\end{array}$ & $\begin{array}{l}\text { *Pita-t João i-otam- } \varnothing . \\
\text { pita-ADV João PART-chegar-ABS } \\
\text { Tradução pretendida: 'O João chegou mesmo' } \\
\text { *João pita-t i-otam- } \varnothing . \\
\text { João pita-ADV PART-chegar-ABS } \\
\text { Tradução pretendida: 'O João chegou mesmo' } \\
\text { *Pita-t i-otam-Ø } \\
\text { pita-ADV PART-chegar-ABS João } \\
\text { Tradução pretendida: 'O João chegou mesmo' }\end{array}$ \\
\hline $\begin{array}{l}\text { typ Antônio } \\
\text { Bigode } \\
\text { 'encontrar } \\
\text { Antônio } \\
\text { Bigode' }\end{array}$ & $\begin{array}{ll}\text { *Pita-t } & \text { João } Ø \text {-naka-typ- } \varnothing \quad \text { Antônio Bigode. } \\
\text { pita-ADV } & \text { João 3-DECL-encontrar-NFUT } \\
\text { Tradução pretendida: 'O João encontrou mesmo o Antônio Bigode' }\end{array}$ \\
\hline
\end{tabular}




\begin{tabular}{|c|c|}
\hline & 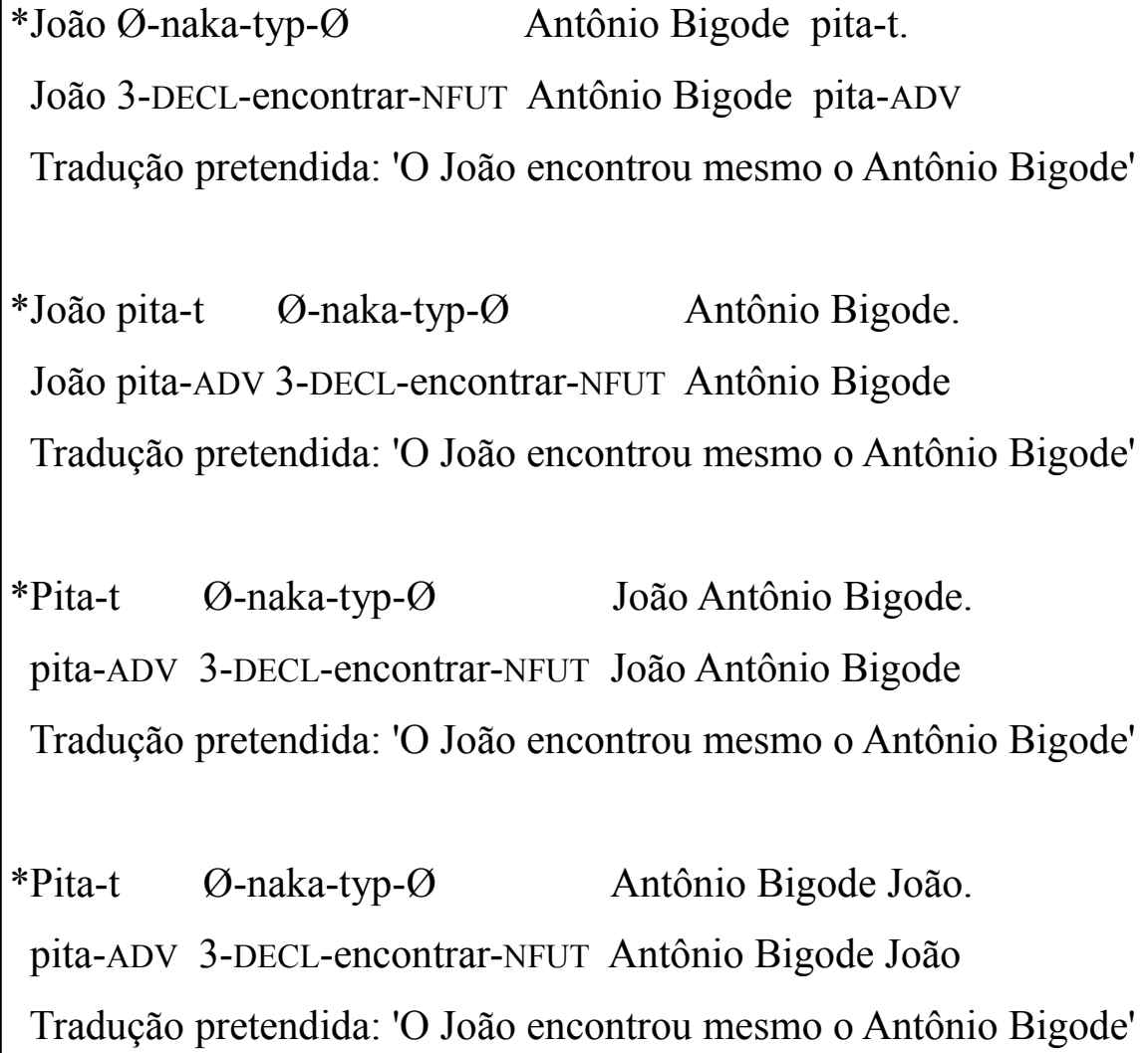 \\
\hline $\begin{array}{l}\text { otam myhint } \\
\text { deso ohyn sok } \\
\text { 'chegar no } \\
\text { topo de uma } \\
\text { montanha' }\end{array}$ & 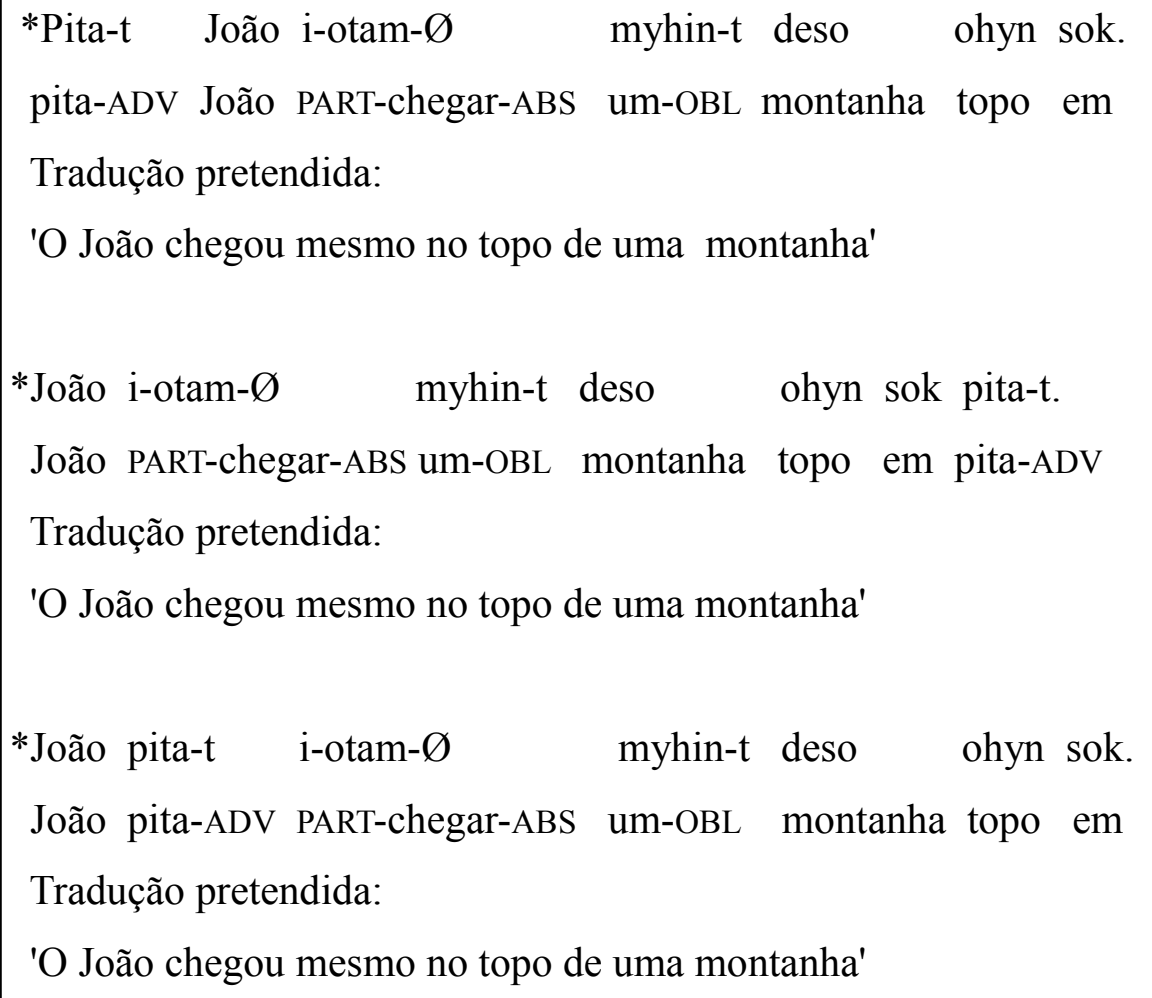 \\
\hline
\end{tabular}




\begin{tabular}{|c|c|}
\hline & 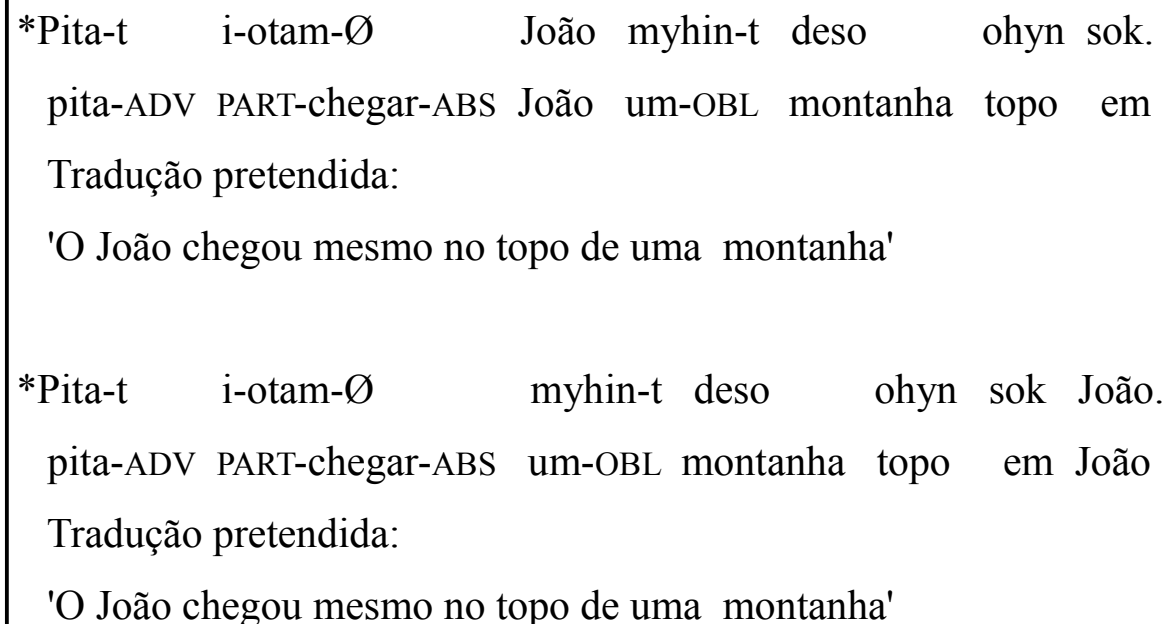 \\
\hline
\end{tabular}

Antes de passar ao significado de pitat com predicados verbais, vamos apresentar a variante fonológica pitadn que ocorre quando há uma consoante que apresenta alofonia nasal em início de palavra depois do advérbio. Os dados abaixo mostram em quais sentenças da tabela de verbos a variante pode ocorrer. As consoantes / $/ g /$ e / $b /$ possuem como alofones $[\eta g]$ e $[\mathrm{mb}]$ nesses ambientes. Vamos continuar usando pitat mesmo nesses contextos porque essa variante não aparece na elicitação de todos os falantes.

a. João Ø-na-pimbik-Ø pita-dn gooj.

João 3-DECL-empurrar-NFUT pita-ADV carro

'O João empurrou muito o carro'

b. João Ø-naka-tagngã-t pita-dn gooj.

João 3-DECL-dirigir-NFUT pita-ADV carro

'O João dirigiu muito o carro'

c. João i-kywytidn- $<\mathrm{a}>-\mathrm{t}$ pita-dn Botỹj-ty.

João PART-acreditar-ABS pita-ADV Deus-OBL

'O João acredita muito em em Deus'

Os dados discutidos acima mostraram que pitat tem uma distribuição bastante comportada no domínio verbal em Karitiana. Ele pode ocorrer com predicados verbais 
atélicos (atividades e estados) e predicados télicos (accomplishments e achievements). No entanto, a distribuição do advérbio com as diferentes classes verbais revela uma diferença de significado. Quando modifica predicados atélicos, pitat tem um significado que se aproxima do muito do português. Já quando modifica predicados télicos, pitat significa algo parecido com uma confirmação da sentença. Essa distribuição e significado dependendo do tipo de predicado faz pitat ser um advérbio bastante diferente dos modificadores de grau verbais estudados na literatura, como a lot 'muito' do inglês, por exemplo.

As sentenças em (248) mostram que a lot tem uma restrição em sua distribuição com predicados verbais. O advérbio pode modificar predicados atélicos, mas torna a sentença agramatical se o predicado é télico.

(248) a. Yanning walked a lot.

'Yanning correu muito'

b. * Yanning ate his pancake a lot.

'Yanning comeu muito sua panqueca'

(exemplos 19 e 22 de Caudal e Nicolas 2005, p. 5)

Além disso, pitat apresenta uma característica singular: quando modifica predicados atélicos, ele pode ser usado em uma gama de situações maior do que a encontrada para sentenças com a lot do inglês ou mesmo o muito do português. As descrições dos contextos abaixo ilustram essa possibilidade. 
Tabela 45: Pitat Modificando Predicados Atélicos (Situações Possíveis)

\begin{tabular}{|c|c|}
\hline Verbo & Exemplo \\
\hline \multicolumn{2}{|r|}{ Atividades } \\
\hline $\begin{array}{l}\text { pykyn } \\
\text { 'correr' }\end{array}$ & $\begin{array}{l}\text { João i-pykyn-<a>-t pita-t. } \\
\text { João PART-correr-ABS pita-ADV } \\
\text { 'O João correu muito' } \\
\text { Situações em que a sentença seria verdadeira: } \\
\text { (i) O João correu por muito tempo (em uma ocasião); } \\
\text { (ii) O João correu em alta velocidade (em uma ocasião); } \\
\text { (iii) O João correu uma longa distância (em uma ocasião); } \\
\text { (iv) O João correu muitas vezes; } \\
\text { (v) O João correu uma vez, com muita intensidade, fazendo muito esforço. }\end{array}$ \\
\hline $\begin{array}{l}\text { pytim'adn } \\
\text { 'trabalhar' }\end{array}$ & $\begin{array}{l}\text { João i-pytim'adn-<a>-t pita-t. } \\
\text { João PART-trabalhar-ABS pita-ADV } \\
\text { 'O João trabalhou muito' } \\
\text { Situações em que a sentença seria verdadeira: } \\
\text { (i) O João trabalhou por muito tempo (em uma ocasião); } \\
\text { (ii) O João trabalhou muitas vezes; } \\
\text { (iii) O João trabalhou arduamente (em uma ocasião). }\end{array}$ \\
\hline $\begin{array}{l}\text { pimbik gooj } \\
\text { 'empurrar } \\
\text { carro' }\end{array}$ & $\begin{array}{l}\text { João Ø-na-pimbik-Ø pita-t gooj. } \\
\text { João 3-DECL-empurrar-NFUT pita-ADV carro } \\
\text { 'O João empurrou muito o carro' } \\
\text { Situações em que a sentença seria verdadeira: } \\
\text { (i) O João empurrou o carro por muito tempo (em uma ocasião); } \\
\text { (ii) O João empurrou o carro em alta velocidade (em uma ocasião); } \\
\text { (iii) O João empurrou o carro por uma longa distância (em uma ocasião); } \\
\text { (iv) O João empurrou o carro muitas vezes; } \\
\text { (v) O João empurrou o carro uma vez, fazendo muito força. }\end{array}$ \\
\hline
\end{tabular}




\begin{tabular}{|c|c|}
\hline $\begin{array}{l}\text { tagngã gooj } \\
\text { 'dirigir carro' }\end{array}$ & $\begin{array}{l}\text { João Ø-naka-tagngã-t pita-t gooj. } \\
\text { João 3-DECL-dirigir-NFUT muito-ADV carro } \\
\text { 'O João dirigiu muito o carro' } \\
\text { Situações em que a sentença seria verdadeira: } \\
\text { (i) O João dirigiu o carro por muito tempo (em uma ocasião); } \\
\text { (ii) O João dirigiu o carro em alta velocidade (em uma ocasião); } \\
\text { (iii) O João dirigiu o carro por uma longa distância (em uma ocasião); } \\
\text { (iv) O João dirigiu o carro muitas vezes. }\end{array}$ \\
\hline & Estativos Stage-level \\
\hline $\begin{array}{l}\text { siki'y } \\
\text { 'querer' }\end{array}$ & $\begin{array}{l}\text { João i-siki'y-t pita-t ese-ty. } \\
\text { João PART-querer-ABS pita-ADV água-OBL } \\
\text { 'O João quis muito água' } \\
\text { Situações em que a sentença seria verdadeira: } \\
\text { (i) O João quis água por muito tempo (em uma ocasião); } \\
\text { (ii) O João quis água muitas vezes; } \\
\text { (iii) O João quis muito água (estava com muita sede). }\end{array}$ \\
\hline $\begin{array}{l}\text { osedn } \\
\text { 'estar alegre' }\end{array}$ & $\begin{array}{l}\text { João i-osedn-Ø pita-t. } \\
\text { João PART-estar.alegre-ABS pita-ADV } \\
\text { 'O João ficou muito alegre' } \\
\text { Situações em que a sentença seria verdadeira: } \\
\text { (i) O João ficou feliz por muito tempo (em uma ocasião); } \\
\text { (ii) O João ficou feliz muitas vezes; } \\
\text { (iii) O João ficou muito alegre / com muita intensidade. }\end{array}$ \\
\hline
\end{tabular}




\begin{tabular}{|c|c|}
\hline $\begin{array}{l}\text { em } \\
\text { 'estar sujo' }\end{array}$ & $\begin{array}{l}\text { João naakat i-em-Ø pita-t. } \\
\text { João PART-estar.sujo-ABS pita-ADV } \\
\text { 'O João ficou muito sujo' } \\
\text { Situações em que a sentença seria verdadeira: } \\
\text { (i) O João ficou sujo por muito tempo (em uma ocasião); } \\
\text { (ii) O João ficou sujo muitas vezes; } \\
\text { (iii) O João ficou muito sujo / com muita intensidade. }\end{array}$ \\
\hline $\begin{array}{l}\text { pasadn } \\
\text { 'gostar' }\end{array}$ & $\begin{array}{l}\text { João i-pasadn-Ø pita-t Milena-ty. } \\
\text { João PART-gostar-ABS pita-ADV Milena-OBL } \\
\text { 'O João gostou muito da Milena' } \\
\text { Situações em que a sentença seria verdadeira: } \\
\text { (i) O João gostou da Milena por muito tempo (em uma ocasião); } \\
\text { (ii) O João gostou da Milena muitas vezes (gostou e deixou de gostar); } \\
\text { (iii) O João gostou muito da Milena / com muita intensidade. }\end{array}$ \\
\hline & Estativo Individual-level (Interpretado como stage-level) \\
\hline $\begin{array}{l}\text { kywytidn } \\
\text { Boty) jty } \\
\text { 'acreditar em } \\
\text { Deus' }\end{array}$ & $\begin{array}{l}\text { João i-kywytidn- }<\text { a }>-t \text { pita-t Botỹj-ty. } \\
\text { João PART-acreditar-ABS pita-ADV Deus-OBL } \\
\text { 'O João acredita muito em em Deus' } \\
\text { Situações em que a sentença seria verdadeira: } \\
\text { (i) O João acreditou em Deus por muito tempo (em uma ocasião); } \\
\text { (ii) O João acreditou em Deus muitas vezes; } \\
\text { (iii) O João acreditou em Deus com muita fé. }\end{array}$ \\
\hline
\end{tabular}

É preciso notar que as situações apresentadas acima descrevem contextos independentes. Não é o caso de que uma das leituras possa ser derivada da outra, como por exemplo, 'O João correu uma longa distância porque correu em alta velocidade'. Cada uma das situações foi checada independentemente, excluindo as outras. Para 'O João correu em alta velocidade', por exemplo, checamos se poderia ter sido por pouco tempo, por uma curta 
distância, em uma ocasião e sem fazer muito esforço (como se o Inácio fosse um super-herói).

Por outro lado, quando pitat é usado com predicados télicos, ele é traduzido normalmente para 'mesmo' em português. Uma elicitação das sentenças com predicados télicos e o advérbio pitat revelou que seu significado nesses conxtextos é o mesmo de uma confirmação de ocorrência do evento. Duas características dessas sentenças apoiam essa hipótese. Primeiramente, muitas vezes, nas sentenças com predicados télicos modificados por pitat, os consultores não traduziam pitat. A tabela abaixo apresenta os dados em que pitat não foi traduzido.

Tabela 46: Pitat Modificando Predicados Télicos (Pitat sem tradução)

\begin{tabular}{|c|c|}
\hline Verbo & Exemplo \\
\hline \multicolumn{2}{|r|}{ Accomplishment } \\
\hline kokot Sete de & pita-t Sete de Setembro \\
\hline Setembro dewota & João PART-passar-ABS pita-ADV Sete de Setembro \\
\hline kyyn & dewota kyyn. \\
\hline 'atravessar a Sete de & outro.lado para \\
\hline Setembro' & 'O João atravessou a rua Sete de Setembro' \\
\hline pog pyejepa goot & \multirow{3}{*}{$\begin{array}{l}\text { João } \varnothing \text {-naka-pog-<a>-t pita-t } \quad \text { pyejepa goot. } \\
\text { João 3-DECL-pintar.de.branco-NFUT pita-ADV } \\
\text { 'O João pintou a escola nova de branco' }\end{array}$} \\
\hline 'pintar a escola nova & \\
\hline de branco' & \\
\hline tat Porto Velho pip & \multirow{3}{*}{$\begin{array}{l}\text { João itat } \quad \text { pita-t } \quad \text { Porto Velho pip. } \\
\text { João PART-ir-ABS } \text { pita-ADV Porto Velho para } \\
\text { 'O João foi para Porto Velho' }\end{array}$} \\
\hline 'ir para Porto Velho' & \\
\hline & \\
\hline 'a myhint boet & \multirow{3}{*}{$\begin{array}{l}\text { João Ø-naka-m-'a-t } \quad \text { pita-t myhin-t boet. } \\
\text { João 3-DECL- CAUS-fazer-NFUT pita-ADV um-OBL colar } \\
\text { 'O João fez um colar' }\end{array}$} \\
\hline 'fazer um colar' & \\
\hline & \\
\hline \multicolumn{2}{|r|}{ Achievement } \\
\hline pop & João i-pop- $\varnothing \quad$ pita-t. \\
\hline 'morrer' & João PART-morrer-ABS pita-ADV \\
\hline & 'O João morreu' \\
\hline
\end{tabular}




\begin{tabular}{|c|c|}
\hline $\begin{array}{l}\text { otam } \\
\text { 'chegar' }\end{array}$ & $\begin{array}{l}\text { João i-otam- } \varnothing \quad \text { pita-t. } \\
\text { João PART-chegar-ABS pita-ADV } \\
\text { 'O João chegou' }\end{array}$ \\
\hline $\begin{array}{l}\text { typ Antônio Bigode } \\
\text { 'encontrar Antônio } \\
\text { Bigode' }\end{array}$ & $\begin{array}{l}\text { João Ø-naka-typ-Ø pita-t Antônio Bigode. } \\
\text { João 3-DECL-encontrar-NFUT pita-ADV Antônio Bigode } \\
\text { 'O João encontrou o Antônio Bigode' }\end{array}$ \\
\hline $\begin{array}{l}\text { otam myhint deso } \\
\text { ohyn sok } \\
\text { 'chegar no topo de } \\
\text { uma montanha' }\end{array}$ & $\begin{array}{l}\text { João i-otam- } \varnothing \text { pita-t myhin-t deso } \\
\text { João PART-chegar-ABS pita-ADV um-OBL montanha topo em } \\
\text { 'O João chegou no topo de uma montanha' }\end{array}$ \\
\hline
\end{tabular}

A segunda característica que apoia a proposta de que pitat modificando predicados télicos significa uma confirmação de que o telos foi atingido está nas sentenças com negação. As sentenças com predicados atélicos modificados por pitat quando são negadas significam algo do tipo 'O João não correu muito' ou 'O João correu pouco', enquando que as senteças com predicados télicos modificados por pitat, quando são negadas, significam que o evento não ocorreu, e não que ele não ocorreu muitas vezes. A tabela abaixo mostra essa característica. A negação em Karitiana ainda não foi analisada em detalhes, mas os dados revelam que as sentenças negativas frequentetemente apresentam concordância negativa. Os dados do capítulo 3 com sapadni 'ainda' são um exemplo. A sentença abaixo foi retirada da tabela 23 do capítulo anterior.

$$
\begin{array}{llll}
\text { João i-pop- } \quad \text { ty-ka-t. } & \text { Aykiri i-pop- }<\text { o }>\text {-padni sa-padni } & \text { i. } \\
\text { João PART-morrer-ABS } & \text { IMP-MOV.SG-NFUT então 3-morrer-NEG } & \text { ainda-NEG } & \text { ele } \\
\text { 'O João está morrendo. Então, ele não morreu ainda' } &
\end{array}
$$

A tabela abaixo mostra que pitat ocorre como pitadni nas sentenças negativas. Por questão de uniformização com outros casos, como o da sentença acima, estamos assumindo que se trata de uma concordância negativa e glosando pitadni como pita-NEG. 
Tabela 47: Predicados Verbais Modificados por Pitat com Negação

\begin{tabular}{|c|c|}
\hline Verbo & Exemplo \\
\hline \multicolumn{2}{|r|}{ Atividades } \\
\hline $\begin{array}{l}\text { pykyn } \\
\text { 'correr' }\end{array}$ & $\begin{array}{l}\text { I-pykyn-<a> pita-dni padni João. } \\
\text { 3-correr pita-NEG NEG João } \\
\text { 'O João não correu muito' } \\
\text { 'O João correu pouco' }\end{array}$ \\
\hline $\begin{array}{l}\text { pytim'adn } \\
\text { 'trabalhar' }\end{array}$ & $\begin{array}{l}\text { I-pytim'adn- }<\text { a }>\text { pita-dni padni João. } \\
\text { 3-trabalhar pita-NEG NEG João } \\
\text { 'O João não trabalhou muito' } \\
\text { 'O João trabalhou pouco' }\end{array}$ \\
\hline $\begin{array}{l}\text { pimbik gooj } \\
\text { 'empurrar carro' }\end{array}$ & $\begin{array}{l}\text { I-pimbik pita-dni padni João gooj. } \\
\text { 3-empurrar pita-NEG NEG João carro } \\
\text { 'O João não empurrou muito o carro' } \\
\text { 'O João empurrou pouco o carro' }\end{array}$ \\
\hline $\begin{array}{l}\text { tagngã gooj } \\
\text { 'dirigir carro' }\end{array}$ & $\begin{array}{l}\text { I-tagngã pita-dni padni João gooj. } \\
\text { 3-dirigir pita-NEG NEG João carro } \\
\text { 'O João não dirigiu muito o carro' } \\
\text { 'O João dirigiu pouco o carro' }\end{array}$ \\
\hline \multicolumn{2}{|r|}{ Estativos Stage-level } \\
\hline $\begin{array}{l}\text { siki'y } \\
\text { 'querer' }\end{array}$ & $\begin{array}{l}\text { I-siki'y pita-dni padni João ese-ty. } \\
\text { 3-querer pita-NEG NEG João água-OBL } \\
\text { 'O João não quis muito água' } \\
\text { 'O João quis um pouco de água' }\end{array}$ \\
\hline $\begin{array}{l}\text { osedn } \\
\text { 'estar alegre' }\end{array}$ & $\begin{array}{l}\text { I-osedn pita-dni padni João. } \\
\text { 3-estar.alegre pita-NEG NEG João } \\
\text { 'O João não ficou muito alegre' } \\
\text { 'O João ficou pouco alegre' }\end{array}$ \\
\hline $\begin{array}{l}\text { em } \\
\text { 'estar sujo' }\end{array}$ & $\begin{array}{l}\text { I-aka pita-dni padni João em. } \\
\text { 3-COP pita-NEG NEG João sujo } \\
\text { 'O João não ficou muito sujo' } \\
\text { 'O João ficou pouco sujo' }\end{array}$ \\
\hline
\end{tabular}




\begin{tabular}{|c|c|}
\hline $\begin{array}{l}\text { pasadn } \\
\text { 'gostar' }\end{array}$ & $\begin{array}{l}\text { I-pasadn pita-dni padni João Milena-ty. } \\
\text { 3-gostar pita-NEG NEG João Milena-OBL } \\
\text { 'O João não gosta muito da Milena' } \\
\text { 'O João gosta pouco da Milena' }\end{array}$ \\
\hline \multicolumn{2}{|r|}{ Estativos Individual-level } \\
\hline $\begin{array}{l}\text { pypyyt } \\
\text { Matematicaty } \\
\text { 'saber Matemática' }\end{array}$ & $\begin{array}{l}\text { I-pypyyt pita-dni padni João Matematica-ty. } \\
\text { 3-saber pita-NEG NEG João Matemática-OBL } \\
\text { 'O João não aprendeu Matemática muito bem' } \\
\text { 'O João aprendeu um pouco de Matemática' }\end{array}$ \\
\hline $\begin{array}{l}\text { kywytidn Botỹjty } \\
\text { 'acreditar em Deus' }\end{array}$ & $\begin{array}{l}\text { I-kywytidn- }<\text { a }>\text { pita-dni padni João Botỹj-ty. } \\
\text { 3-acreditar pita-NEG NEG João Deus-OBL } \\
\text { 'O João não acredita muito em Deus' } \\
\text { 'O João acredita pouco em Deus' }\end{array}$ \\
\hline \multicolumn{2}{|r|}{ Accomplishment } \\
\hline $\begin{array}{l}\text { kokot Sete de } \\
\text { Setembro dewota } \\
\text { kyyn } \\
\text { 'atravessar a Sete de } \\
\text { Setembro' }\end{array}$ & $\begin{array}{l}\text { I-kokot pita-dni padni João Sete de Setembro dewota kyyn. } \\
\text { 3-passar pita-NEG NEG João Sete de Setembro outro.lado para } \\
\text { 'O João não atravessou a rua Sete de Setembro' } \\
\text { Situação: começou, mas não conseguiu atravessar }\end{array}$ \\
\hline $\begin{array}{l}\text { pog pyejepa goot } \\
\text { 'pintar a escola nova } \\
\text { de branco' }\end{array}$ & $\begin{array}{l}\text { I-pog-<a> pita-dni padni João pyejepa goot. } \\
\text { 3-pintar.de.branco pita-NEG NEG João escola novo } \\
\text { 'O João não pintou a escola nova de branco completamente' } \\
\text { Situação: pintou meia parede e parou }\end{array}$ \\
\hline $\begin{array}{l}\text { tat Porto Velho pip } \\
\text { 'ir para Porto Velho' }\end{array}$ & $\begin{array}{l}\text { I-tat pita-dni padni João Porto Velho pip. } \\
\text { 3-ir pita-NEG NEG João Porto Velho para } \\
\text { 'O João não foi para Porto Velho' } \\
\text { Situação: ele foi, mas não chegou, acabou a gasolina do seu carro }\end{array}$ \\
\hline $\begin{array}{l}\text { 'a myhint boet } \\
\text { 'fazer um colar' }\end{array}$ & $\begin{array}{l}\text { I-m-'a pita-dni padni João myhint boet. } \\
\text { 3-CAUS-fazer pita-NEG NEG João um-OBL colar } \\
\text { 'O João não fez um colar' } \\
\text { Situação: fez um colar incompleto }\end{array}$ \\
\hline
\end{tabular}




\begin{tabular}{|l|l|}
\hline \multicolumn{2}{|c|}{ Achievement } \\
\hline pop & $\begin{array}{l}\text { I-pop pita-dni padni João. } \\
\text { 3-morrer pita-NEG NEG João } \\
\text { 'O João não morreu' }\end{array}$ \\
\hline otam & $\begin{array}{l}\text { I-otam pita-dni padni João. } \\
\text { 'chegar' }\end{array}$ \\
3-chegar pita-NEG NEG João \\
'O João não chegou' \\
typ Antônio Bigode & $\begin{array}{l}\text { I-typ pita-dni padni João Antonio Bigode. } \\
\text { 3-encontrar pita-NEG NEG João Antônio Bigode } \\
\text { Bigode' }\end{array}$ \\
\hline $\begin{array}{l}\text { otam myhint deso } \\
\text { ohyn sok }\end{array}$ & $\begin{array}{l}\text { I-otam pita-dni padni João myhin-t deso } \\
\text { 3-chegar pita-NEG NEG João um-OBL montanha topo em } \\
\text { 'chegar no topo de } \\
\text { uma montanha' }\end{array}$ \\
\hline
\end{tabular}

Dada a descrição apresentada acima, uma análise apropriada para pitat modificando sintagmas verbais deve explicar sua diferença de siginificado quando modifica predicados télicos e atélicos. Na próxima seção, apresentamos nossa análise para essa propriedade de pitat que leva em conta graus e escalas indeterminadas ${ }^{96}$.

\subsubsection{Graus e Escalas Associados aos Eventos}

O objetivo desta seção é aproximar a teoria de graus descrita na seção 2 deste capítulo à Semântica de Eventos apresentada no capítulo 1. Embora a noção de grau tenha sido tratada primeiramente associada aos adjetivos, ela também pode ser atribuída a outros termos como verbos e nomes (Kennedy e McNally 2005). Essa característica já havia sido apontada por Sapir em seu trabalho clássico sobre gradação (Sapir 1944), mas vinha sendo negligenciada nos estudos sobre o assunto. Mais recentemente, no entanto, muitos trabalhos têm explorado o papel do grau e das estruturas escalares em construções verbais. Alguns exemplos são Rappaport Hovav e Levin (2010), Hay, Kennedy e Levin (1999), Kennedy e Levin (2008) e Caudal e Nicolas (2005).

\footnotetext{
${ }_{96} \mathrm{O}$ termo escalas indeterminadas será explicado mais abaixo.
} 
Entretanto, esses trabalhos estavam interessados sobretudo na investigação de predicados verbais télicos (achievements e accomplishments) e de como a telicidade pode ser descrita em termos escalares. No entanto, propomos que o instrumental utilizado pelos trabalhos interessados na investigação de predicados verbais télicos pode ser aplicado adequadamente também na modificação de predicados atélicos pelo advérbio pitat do Karitiana. O objetivo é mostrar que uma teoria semântica que leva em conta estruturas escalares, embora tenha sido construída para dar conta de predicados télicos, é o modelo apropriado para se analisar a modificação de grau de predicados verbais télicos e atélicos das sentenças do Karitiana.

Conforme descrito na seção 2 deste capítulo, há uma divisão das estruturas escalares entre escalas abertas e fechadas. Retomando brevemente, escalas abertas são aquelas que não possuem grau máximo e/ou mínimo, e escalas fechadas são aquelas que possuem. Caudal e Nicolas (2005) aplicaram essa distinção, que foi amplamente empregada no estudos dos adjetivos, aos predicados verbais. Segundo os autores, predicados verbais télicos, uma vez que possuem um limite final definido pelo telos, podem ser considerados predicados de escala fechada e, por outro lado, predicados verbais atélicos, como não possuem um ponto final, podem ser aproximados dos predicados de escala aberta. Essa associação está apresentada na tabela abaixo:

Tabela 48: Relação entre Predicados Verbais e Tipos de Escalas

\begin{tabular}{|l|l|}
\hline Tipo de Predicado Verbal & \multicolumn{1}{c|}{ Tipo de Escala } \\
\hline Télico & Fechada \\
\hline Atélico & Aberta \\
\hline
\end{tabular}

Uma vez que pitat pode ser utilizado com predicados verbais télicos e atélicos, podese dizer que ele é um modificador de grau que não apresenta uma restrição em seu domínio, podendo ocorrer com predicados de escala aberta ou fechada. Essa distribuição é diferente, por exemplo, do very 'muito' do inglês, que só pode modificar adjetivos de escala aberta (Kennedy e McNally 2005); e do a lot 'muito' que só pode ser usado com predicados verbais de escala aberta (atélicos) (Caudal e Nicolas 2005), discutidos anteriormente.

A distribuição irrestrita de pitat com os predicados verbais o aproxima do beaucoup 
'muito' do francês, discutido no capítulo 2. Conforme apresentado ateriormente, beaucoup, assim como pitat, pode ser usado irrestritamente com predicados télicos e atélicos, mas sua interpretação é dependente do tipo de predicado (Doetjes 2007). Quando usado com predicados télicos, beaucoup tem uma interpretação iterativa, de 'muitas vezes', e quando usado com predicados atélicos, apresenta uma leitura de intensidade.

(250) a. Sylvie va beaucoup au cinema.

'Sylvie vai muitas vezes ao cinema'

(exemplo 1a de Doetjes 2007, p. 1)

b. Il a plu beaucoup.

'Choveu intensamente'

(exemplo 2a de Doetjes 2007, p. 2)

A semelhança entre pitat e beaucoup, no entanto, está apenas na existência de uma relação entre a interpretação do advérbio e o tipo de verbo modificado. Pitat, como se viu, não tem uma interpretação iterativa quando modifica predicados télicos, mas uma leitura de confirmação. Vamos apresentar uma proposta que dê conta tanto dessa leitura de confirmação $\mathrm{cm}$ predicados télicos quando da leitura de um grau acima do normal associado a várias escalas com predicados atélicos.

Intuitivamente, há uma diferença entre a modificação de grau no domínio adjetival e no domínio verbal. Quando se diz A Maria é muito bonita, está claro que a dimensão da escala envolvida na interpretação da sentença é dada pelo significado lexical do adjetivo: a sentença é avaliada em relação a uma escala de beleza. No entanto, em sentenças como O homem trabalhou muito ou, em sua versão em Karitiana, Taso ipytim'adnat pitat, a escala apropriada para avaliação das condições de verdade da sentença não é óbvia, mas deve ser buscada no contexto.

Essa intuição é adequadamente capturada pela teoria semântica que assume graus e estruturas escalares. As funções de medida que representam as escalas em construções com adjetivos estão disponíveis por meio do léxico (Kennedy 1999, Kennedy e McNally 2005). É por isso que normalmente há uma correspondência entre os adjetivos e as escalas a eles relacionadas. $\mathrm{O}$ adjetivo alto, por exemplo, está associado à escala de altura; já o adjetivo feliz 
à escala de felicidade; e assim por diante. Mesmo quando não há uma semelhança lexical, a função de medida é facilmente recuperável: cheio e vazio são avaliados em uma escala de ocupação, por exemplo. Essa característica aparece formalmente na entrada lexical dos adjetivos graduáveis assumida pela Semântica Escalar. Como se vê em (251a), um adjetivo é avaliado segundo uma escala definida pela função de medida $\mathbf{g}_{\text {adj }}$ associada a ele. Em (251b), há um exemplo com o adjetivo alto.

$$
\begin{array}{ll}
\text { a. } & \llbracket A d j_{g} \rrbracket=\lambda \mathrm{d} \lambda \mathrm{x} . \mathrm{g}_{\text {adj }}(\mathrm{x})=\mathrm{d} \\
\text { b. } & \llbracket \text { alto } \rrbracket=\lambda \mathrm{d} \lambda \mathrm{x} . \text { "altura" }(\mathrm{x})=\mathrm{d}
\end{array}
$$

$\mathrm{Na}$ entrada lexical dos adjetivos graduáveis pode-se ver, claramente, de que modo essa teoria formaliza a relação intrínseca entre graus e escalas. O grau entra na denotação na forma de um argumento de tipo d, o tipo dos graus. Desse modo, adjetivos graduáveis, que eram tratados tradicionalmente pela Semântica Formal como de tipo $<\mathrm{e}, \mathrm{t}>$, passam a ser de tipo $<\mathrm{d},<\mathrm{e}, \mathrm{t}>>$. A escala aparece na denotação do adjetivo como qualificadora do argumento nominal de tipo e.

Já os verbos de atividade, como o verbo trabalhar, não codificam lexicalmente uma escala (cf. Rappaport Hovav e Levin 2010). Não há uma escala "trabalharidade” lexicalmente associada ao predicado trabalhar. Isso não quer dizer, no entanto, que as construções verbais desse tipo não possam ser relacionadas a uma escala. A proposta adotada neste trabalho é a de que as escalas, nesse caso, em vez de estarem disponíveis no léxico, são construídas contextualmente.

Assumimos que os VPs de atividade e estativos stage-level (ou individual-level que se comportam como stage-level quando modificados) são predicados que denotam eventos que podem estar associados a várias dimensões possíveis. A variedade de dimensões associadas aos predicados é responsável pela variedade de escalas relacionadas a eles e, portanto, pela indeterminação na leitura das sentenças.

No caso de trabalhar, as escalas podem ser, por exemplo, tempo de duração, número de ocorrências ou intensidade. Desse modo, quando se diz que João ipytim'adnat pitat 'O João trabalhou muito', pode-se estar querendo dizer que o João trabalhou por muitas horas (escala de duração), ou que trabalhou muitas vezes (escala de número de ocorrências), ou ainda que trabalhou com muito empenho (escala de intensidade). Do mesmo modo, quando se diz que o 
João trabalhou mais do que a Luciana, pode-se estar querendo dizer que ele trabalhou por mais tempo, mais vezes, ou com mais empenho do que ela. No caso de verbos de movimento, como correr, outras escalas podem ser construídas, como velocidade e distância.

A variedade de escalas associadas a esses predicados está disponível no instrumental formal da Semântica Escalar por uma característica que Kennedy e McNally (2005) chamam de indeterminação das escalas. A indeterminação é a possibilidade de um predicado ser compatível com escalas de várias dimensões, que são estabelecidas pelo contexto. Os vários aspectos do significado de um verbo que podem ser medidos (como extensão temporal, número de ocorrências, velocidade, intensidade, etc.) podem ser usados para fixar os parâmetros das dimensões das escalas. $\mathrm{O}$ fato de a iteratividade poder ser uma dessas escalas é uma evidência de que o domínio verbal em Karitiana é todo contável, conforme defendido no capítulo 3. Segundo Doetjes (2007) e Bach (1986), predicados atélicos podem ser considerados como predicados massivos. O capítulo 3 mostrou que, em Karitiana, os predicados atélicos se comportam como os télicos no que diz respeito à contabilidade, apoiando a proposta de Rothstein $(1999,2004,2008)$.

Conforme apresentado acima, dados com predicados atélicos em Karitiana apresentam uma gama de situações em que a sentença é verdadeira. A sentença (252) apresenta outro verbo do mesmo tipo, que também apresenta essa variedade de interpretações relacionadas à duração, velocidade, distância, iteratividade, e intensidade, que é associada, pelos falantes, ao prazer $^{97}$.

(252) Milena i-tarak-<a $>$-t pita-t.

Milena PART-andar-ABS pita-ADV

'A Milena andou muito'

(253) Situações em que a sentença (252) seria verdadeira:
a. 'A Milena andou por muito tempo'
b. 'A Milena andou muito rápido'
c. 'A Milena andou uma longa distância'
d. 'A Milena andou muitas vezes'
e. 'A Milena andou com muito gosto'

97 As interpretações relacionadas a empenho, vibração e prazer estão sendo analisadas como intensidade. 
A nossa proposta é, portanto, a de que as escalas associadas aos verbos atélicos não estão disponíveis pelo léxico, mas são capturadas pela indeterminação das escalas que podem estar associadas ao predicado. Isso quer dizer que quando pitat modifica predicados atélicos, ele opera em escalas dadas contextualmente. Sua leitura é de um grau acima do normal nas escalas que podem estar associadas ao predicado.

Por outro lado, quando pitat modifica predicados télicos ele tem um significado de confirmação e não está associado a escalas indeterminadas relacionadas ao predicado. Conforme foi apresentado acima, predicados verbais télicos podem ser considerados como predicados de escala fechada. Quando pita é usado para modificar adjetivos de escala fechada, ele seleciona o grau máximo da escala associada ao adjetivo, gerando um significado de completamente. Esse é o mesmo processo encontrado nos predicados télicos modificados por pitat. O advérbio também seleciona o grau máximo. O grau máximo da escala desses predicados é o telos do evento denotado por eles. Ou seja, quando pitat é usado com um predicado télico, ele significa que o telos do evento foi atingido. Daí sua tradução mais usual para 'mesmo' do português. Conforme ilustrada acima, em alguns casos, os consultores nem traduzem pitat nesses contextos porque sua contribuição semântica é de que o telos do evento denotado pelo verbo foi atingido, e o tempo pretérito perfeito do português usualmente já desempenha essa função ${ }^{98}$.

Uma vez apresentada de modo geral qual a nossa proposta para a modificação de predicados verbais por pitat, vamos mostrar de que forma essa proposta pode ser implementada formalmente. Conforme apresentado no capítulo 1, os predicados verbais são entendidos como predicados de eventos. A denotação do verbo ferir, foi apresentada como sendo a relação de um evento de ferir com o indivíduo ferido (denotação repetida abaixo) ${ }^{99}$ :

Para que um predicado verbal possa ser modificado por um modificador de grau, ele precisa de um argumento de grau $(\boldsymbol{\lambda} \mathbf{d})$. Como vimos, no caso dos adjetivos graduáveis, esse argumento já faz parte da denotação do predicado (denotação em 255):

98 Basso (2007) problematiza essa questão para o português, mostrando que o passado perfeito nem sempre implica que o telos de um evento foi atingido.

99 Conforme apresentado no capítulo 1, estamos assumindo a proposta de Kratzer (1996) de que o argumento externo não faz parte da denotação do verbo e é inserido sintaticamente por uma núcleo voice. 
Para formalizar a interpretação de pitat no domínio verbal, assumimos que os predicados verbais podem ganhar um argumento de grau durante a derivação semântica (Caudal e Nicolas 2005). Caudal e Nicolas (2005) assumem que os predicados podem ter um argumento de grau e, consequentemente, uma escala a eles associada por meio de: (i) definição lexical; ou (ii) em virtude do contexto sintático ou semântico. Para formalizar essa segunda possibilidade, Piñón (2000) afirma que é possível fornecer um argumento de grau para os predicados durante o curso da derivação semântica por meio de um modificador (como more and more 'mais e mais') ou por meio de uma função de grau.

Embora este trabalho esteja analisando um modificador, a sua formalização não pode ser exatamente como a proposta por Piñón (2000) para more and more. Na proposta do autor, quando um modificador como more and more é usado, a escala relevante na qual a sentença deve ser avaliada é construída automaticamente por estar presente na sentença. Na sentença (256) abaixo, por exemplo, more and more 'mais e mais' seleciona o advérbio quickly 'rapidamente' e já fornece a escala de avaliação, a escala de velocidade.

(256) Mary ran more and more quickly.

'A Maria correu mais e mais rápido'

Conforme apresentado acima, na modificação de grau de predicados atélicos por pitat, a escala nunca é fornecida explicitamente na sentença. Isso indica que os predicados têm que ganhar não apenas um argumento de grau, mas também uma variável que representa suas escalas indeterminadas. Logo, não é sensato implementar a proposta de Piñón (2000) de inserção do argumento de grau via um modificador. A outra sugestão do autor para inserir um argumento de grau em predicados que lexicalmente não o possuem é por meio de uma função de grau. A condição de aplicabilidade da função é descrita abaixo: 
(257) Seja $\delta$ uma função de (triplas de) eventos e, objetos $\mathrm{x}$, e relações $\mathrm{R}$ entre eventos e objetos a graus d. $\delta$ é uma função de grau se, e somente se:

$\mathrm{R}(\mathrm{e}, \mathrm{x}) \& \forall \mathrm{x}^{\prime}\left[\mathrm{R}\left(\mathrm{e}, \mathrm{x}^{\prime}\right) \rightarrow \mathrm{x}^{\prime}=\mathrm{x}\right] \& \forall \mathrm{e}^{\prime}\left[\mathrm{e}^{\prime} \subseteq\right.$ ini $\left.\mathrm{e} \rightarrow \exists \mathrm{x}^{\prime}\left[\mathrm{x}^{\prime} \subset \mathrm{x} \& \mathrm{R}\left(\mathrm{e}^{\prime}, \mathrm{x}^{\prime}\right)\right]\right] \leftrightarrow \exists \mathrm{d}$ $[\delta(e, x, R)=d]$

Em palavras: uma relação $\mathbf{R}$ é definida como uma relação entre eventos $\mathbf{e}$ e indivíduos $\mathbf{x}$ em que, para todo $\mathbf{x}^{\prime}$ que entra na relação $\mathbf{R}$, então $\mathbf{x}^{\prime}$ é igual a $\mathbf{x}$, e para todo evento $\mathbf{e}^{\prime}$ se $\mathbf{e}^{\prime}$ é uma parte inicial do evento e então existe um $\mathbf{x}^{\prime}$ que é parte de $\mathbf{x}$ e que entra na relação $\mathbf{R}$ com $\mathbf{e}^{\mathbf{\prime}}$ se, e somente, se existe um grau $\mathbf{d}$ que é resultado da função de grau aplicada ao evento e, ao indivíduo $\mathbf{x}$ e à relação $\mathbf{R}$.

Basicamente, o que a fórmula (257) expressa é a condição de aplicabilidade da função de grau $\boldsymbol{\delta}$. Essa função só pode ser aplicada a verbos em que há uma relação incremental entre os objetos e os eventos, ou seja, verbos de accomplishment. $\mathrm{O}$ argumento de grau entra na denotação como mais uma variável dessa relação. A fórmula em (258) mostra o resultado da aplicação da função $\boldsymbol{\delta}$.

$[\delta(\mathrm{e}, \mathrm{x}, \mathrm{R})=\mathrm{d}] \rightarrow \forall \mathrm{e}^{\prime} \forall \mathrm{x}^{\prime}\left[\mathrm{e}^{\prime} \subseteq\right.$ ini $\left.\mathrm{e} \quad \& \quad \mathrm{x}^{\prime} \subset \mathrm{x} \quad \& \mathrm{R}\left(\mathrm{e}^{\prime}, \mathrm{x}^{\prime}\right)\right] \rightarrow \exists \mathrm{d}^{\prime}\left[\delta\left(\mathrm{e}^{\prime}, \mathrm{x}^{\prime}, \mathrm{R}\right)=\right.$ $\left.\mathrm{d}^{\prime} \& \mathrm{~d}^{\prime}<\mathrm{d}\right]$

Em palavras: se a função de grau $\boldsymbol{\delta}$ aplicada ao evento $\mathbf{e}$, ao indivíduo $\mathbf{x}$ e à relação $\mathbf{R}$

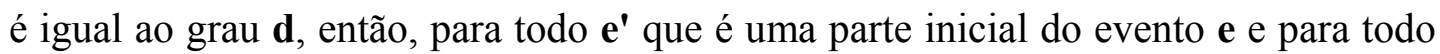
$\mathbf{x}^{\prime}$ que é parte de $\mathbf{x}$, e $\mathbf{e}^{\prime}$ e $\mathbf{x}^{\prime}$ entram na relação $\mathbf{R}$, então existe um grau $\mathbf{d}^{\prime}$ que é resultado da aplicação da função de grau $\boldsymbol{\delta}$ sobre o evento $\mathbf{e}^{\prime}$, o indivíduo $\mathbf{x}^{\prime}$ e a relação $\mathbf{R}$ e esse grau d' é menor do que o grau $\mathbf{d}$.

A representação em (258) mostra que os graus inseridos pela função $\boldsymbol{\delta}$ entram na relação incremental entre objetos e eventos. Esse é exatamente o resultado desejado pelo autor, que está analisando o modificador de grau gradually 'gradualmente' em sentenças como (259) abaixo. 
(259) The disease gradually destroys the motor neurons.

'A doença destrói gradualmente os neurônios motores'

(exemplo 28 de Piñón 2000, p. 11)

A relação entre eventos, indivíduos e graus proposta em (257) e (258) captura adequadamente a relação entre o evento, o objeto e os graus da sentença (259). O grau de destruição mede a quantidade de indivíduos destruídos que é dados pela medida do evento de destruição. Isso quer dizer que quanto mais o evento se desenvolve, mais neurônios são destruídos e maior é o grau de destruição. Essa relação mútua é garantida pela relação $\mathbf{R}$ descrita em (257) e (258).

No entanto, essa função de grau não é adequada para fornecer um grau aos predicados modificados por pitat analisados nesta tese. Os predicados verbais que são modificados por pitat não possuem necessariamente uma relação entre o evento e o objeto como os VPs de accomplishment analisados por Piñón (2000).

Para inserir ao mesmo tempo uma variável de grau e uma escala, vamos propor uma função Deg que toma um predicado de eventos de tipo $\langle\mathrm{s}, \mathrm{t}\rangle$ e devolve uma relação entre graus e eventos do tipo $<\mathrm{d},<\mathrm{s}, \mathrm{t}>>$. Para capturar a indeterminação das escalas, uma função de medida $\boldsymbol{\mu}$ é utilizada como uma variável de dimensões (cf. Krifka 1998, Nakanishi 2007, Thomas 2009). Thomas (2009) utiliza a função $\boldsymbol{\mu}$ para dar conta dos usos das estruturas comparativas da língua Mbyá Guarani em diferentes categorias sintáticas. Sua ideia pode ser adaptada adequadamente aqui para representar a vagueza na interpretação de uma mesma sentença com pitat, representada pela indeterminação da escala.

$$
\llbracket \operatorname{Deg}_{\text {intr }} \rrbracket=\lambda \mathrm{P}_{<\mathrm{s}, \triangleright} \lambda \mathrm{d} \lambda \mathrm{e} . \mathrm{P}(\mathrm{e}) \& \mu(\mathrm{e})=\mathrm{d}
$$

Essa é uma fórmula para VPs intransitivos inergativos. A denotação de Deg para transitivos e inacusativos é fácil de ser deduzida. Ela toma predicados de tipo $<\mathrm{e},<\mathrm{s}, \mathrm{t}>>\mathrm{e}$ devolve um predicado de tipo $<$ d, $<$ e, $<$ s,,$>>>$.

$$
\llbracket \operatorname{Deg}_{\text {trans }} \rrbracket=\lambda \mathrm{P}_{<\mathrm{e},<\mathrm{s},>>} \lambda \mathrm{d} \lambda \mathrm{x} \lambda \mathrm{e} . \mathrm{P}(\mathrm{x})(\mathrm{e}) \& \mu(\mathrm{e})=\mathrm{d}
$$


Uma vez que em Karitiana todos os verbos intransitivos se comportam como verbos inacusativos (Rocha 2011a, 2012), vamos utilizar apenas a denotação (261) da função Deg. A diferença crucial da função de grau proposta em (260) e (261) e a sugerida em Piñón (2000) é a de que a função proposta pelo autor relaciona eventos, objetos e graus com o objetivo de capturar formalmente como um grau pode estar associado ao evento e ao objeto denotados por predicados de accomplishment com tema incremental. A fórmula apresentada em (261) não faz nenhuma representação da relação entre objetos, eventos e graus, apenas da relação entre eventos e objetos $(\mathbf{P}(\mathbf{x})(\mathbf{e}))$. Seu objetivo é fornecer aos predicados verbais um argumento de grau e uma variável sobre escalas para que eles possam ser modificados por pitat.

A função Deg transforma um predicado comum, que não possui um argumento de grau, em um predicado graduável. É preciso notar que o modo como se está utilizando o termo verbo graduável neste trabalho é diferente do modo como é utilizado nos trabalhos sobre a relação entre eventos e graus. Em geral, os verbos ditos graduáveis são os degree achievements como esfriar, derreter ou os accomplishments com tema incremental como comer o bolo. No entanto, neste trabalho, verbo graduável é aquele que passou pela função Deg e ganhou um argumento de tipo d e é, portanto, passível de ser modificado por grau. Por essa razão, passa a ser um predicado graduável.

O modo como a variável sobre escalas $\boldsymbol{\mu}$ é preenchida é que vai derivar a diferença de significado na modificação de predicados télicos e atélicos. Nossa proposta é a de que a indeterminação escalar é uma característica apenas dos predicados atélicos. Nos predicados télicos, a variável sobre escalas é preenchida por uma escala fechada que representa o percurso do evento e é dada pelo léxico. Essa proposta está baseada na proposta de Rothstein (2008) para a atomicidade dos eventos denotados pelos predicados verbais discutida no capítulo 3. Segundo a autora, a denotação de um predicado verbal é a representada em (262).

$$
\llbracket \mathrm{V} \rrbracket=\lambda \mathrm{e} . \mathrm{P}(\mathrm{e}) \wedge \operatorname{MEAS}(\mathrm{e})=<1, \mathrm{U}>
$$

(representação 21 de Rothstein 2008, p.13)

Segundo Rothstein (2008), a denotação de um verbo é um predicado de eventos tal que a medida do evento é dada segundo uma unidade $\mathbf{U}$. Esse critério de atomicidade $\mathbf{U}$ é preenchido de acordo com o tipo de predicado envolvido. Se o predicado for télico, o critério de individuação é dado pelo léxico; se o predicado for atélico, o critério de individuação é 
dado pelo contexto. Os exemplos abaixo ilustram essa diferença.

(263) a. $\quad \llbracket$ chegar $\rrbracket=\lambda$ e. $\mathrm{P}(\mathrm{e}) \wedge \operatorname{MEAS}(\mathrm{e})=<1$, chegada $>$

b. $\quad \llbracket$ correr $\rrbracket=\lambda$ e. $\mathrm{P}(\mathrm{e}) \wedge \operatorname{MEAS}(\mathrm{e})=<1, \mathrm{U}>$

Adotamos a proposta de Rothstein (2008) para o preenchimento do critério de individução dos eventos no preenchimento da variável de escalas $\boldsymbol{\mu}$. Em predicados télicos, a escala é especificada pelo léxico, e em predicados atélicos ela é preenchida contextualmente. Segundo nossa proposta, um verbo como otam 'chegar' pode ter uma forma graduável $\left(\mathrm{V}_{\mathrm{deg}}\right)$ que difere de sua forma original pela presença de um argumento a mais, o argumento de grau, e pela presença de uma escala. Para efeitos de comparação, mostramos em (264a) a denotação do verbo otam 'chegar' em sentenças sem modificação de grau; e em (264b) a denotação do verbo de tipo graduável, depois que a função Deg é aplicada. Em (264b), a escala "chegar" é o resultado da transformação do evento de chegar em uma escala fechada. O polo máximo dessa escala é o telos denotado pelo evento.

$$
\begin{aligned}
& \text { a. }[[\text { otam }]]=\lambda \mathrm{x} \lambda \text { e. chegar }(\mathrm{x})(\mathrm{e}) \\
& \text { b. }\left[\begin{array}{rl}
\left.\left[\text { otam }_{\mathrm{deg}}\right]\right] & =[[\operatorname{Deg}]]([[\text { otam }]]) \\
& =[\lambda \mathrm{P} \lambda \mathrm{d} \lambda \mathrm{x} \lambda \mathrm{e} \cdot \mathrm{P}(\mathrm{x})(\mathrm{e}) \& \mu(\mathrm{e})=\mathrm{d}](\lambda \mathrm{x} \lambda \mathrm{e} \cdot \operatorname{chegar}(\mathrm{x})(\mathrm{e})) \\
& =\lambda \mathrm{d} \cdot \lambda \mathrm{x} \cdot \lambda \text { e. } \operatorname{chegar}(\mathrm{x})(\mathrm{e}) \& \text { "chegar" }(\mathrm{e})=\mathrm{d}
\end{array}\right.
\end{aligned}
$$

Já um verbo como pykyn 'correr' pode ter a seguinte forma graduável.

$$
\begin{aligned}
& \text { a. } \quad[[p y k y n]]=\lambda \mathrm{x} \lambda \mathrm{e} \cdot \operatorname{correr}(\mathrm{x})(\mathrm{e}) \\
& \text { b. } \quad \begin{aligned}
{\left[\left[p y k y n_{\mathrm{deg}}\right]\right] } & =[[\operatorname{Deg}]([[p y k y n]]) \\
& =[\lambda \mathrm{P} \lambda \mathrm{d} \lambda \mathrm{x} \lambda \mathrm{e} \cdot \mathrm{P}(\mathrm{x})(\mathrm{e}) \& \mu(\mathrm{e})=\mathrm{d}](\lambda \mathrm{x} \lambda \mathrm{e} . \operatorname{correr}(\mathrm{x})(\mathrm{e}) \\
& =\lambda \mathrm{d} \lambda \mathrm{x} \lambda \mathrm{e} \cdot \operatorname{correr}(\mathrm{x})(\mathrm{e}) \& \mu(\mathrm{e})=\mathrm{d}
\end{aligned}
\end{aligned}
$$

Em (265b), a função $\boldsymbol{\mu}$ pode ser substituída por tempo de duração, velocidade, distância, número de ocorrências ou intensidade. O número de ocorrência é uma escala livremente disponível porque todos os predicados verbais são contáveis, conforme defendido no capítulo 3. Um possível problema que poderia ser apontado sobre a função Deg é que ela 
poderia gerar mais interpretações do que o necessário. Está claro que nem todos os tipos de verbos vão ter todas as possibilidades de leituras. Por exemplo, não é desejável que seja gerada uma leitura do tipo 'A Luciana acreditou em Deus em alta velocidade'. Mas, nesse caso, o que proíbe esse tipo de leitura de ser gerada é a mesma restrição que não permite que essa seja uma boa sentença em português. A mesma incompatibilidade semântica que evita sentenças do tipo $O$ Inácio ficou feliz por dois quilômetros vai evitar que a leitura 'O Inácio ficou feliz por uma longa distância' da sentença em Karitiana Inácio naakat iosedn pitat ('O Inácio ficou muito feliz').

Finalmente, propomos a seguinte entrada lexical para o modificador de grau pitat:

$$
\llbracket \text { pitat } \rrbracket=\lambda \mathrm{G}_{<\mathrm{d},<\mathrm{e},<\mathrm{s}, \mathrm{\gg}>>} \lambda \mathrm{x}_{\mathrm{e}} \lambda \mathrm{e}_{\mathrm{s} .} \exists \mathrm{d}\left[\mathrm{G}(\mathrm{d})(\mathrm{x})(\mathrm{e}) \& \mathrm{~d} \geq \mathrm{d}_{\mathrm{s}}\right]
$$

Em palavras, pitat é uma função que pede um argumento graduável $\mathbf{G}$ de tipo $<\mathrm{d},<\mathrm{e}<\mathrm{s}, \mathrm{t}>>>$ (o verbo graduável), um argumento de indivíduos $\mathbf{x}$ de tipo e e um argumento de eventos e de tipo $\mathbf{s}$ e devolve uma sentença que aplica a função graduável em $\mathbf{d}, \mathbf{x}$ e e e afirma que existe um grau $\mathbf{d}$ que é maior ou igual ao grau $\mathbf{d}_{\mathrm{s}}$. Assim como no caso dos adjetivos, na fórmula em (266), $\mathbf{d}_{\mathbf{s}}$ representa o grau relevante em cada tipo de predicado. Se for um predicado de escala aberta (atélico), $\mathbf{d}_{\mathbf{s}}$ representa o grau (acima do) normal da escala; se o predicado for de escala fechada, $\mathbf{d}_{\text {s }}$ representa o grau máximo da escala.

Mostramos abaixo dois exemplos de derivação com pitat modificando predicados verbais. Primeiramente com um predicado de escala fechada otam 'chegar' e, em seguida com um predicado de escala aberta pykyn 'correr'.

$$
\begin{aligned}
& \text { João i-otam- } \varnothing \quad \text { pita-t. } \\
& \text { João PART-chegar-ABS pita-ADV } \\
& \text { 'O João chegou mesmo' }
\end{aligned}
$$


(268)

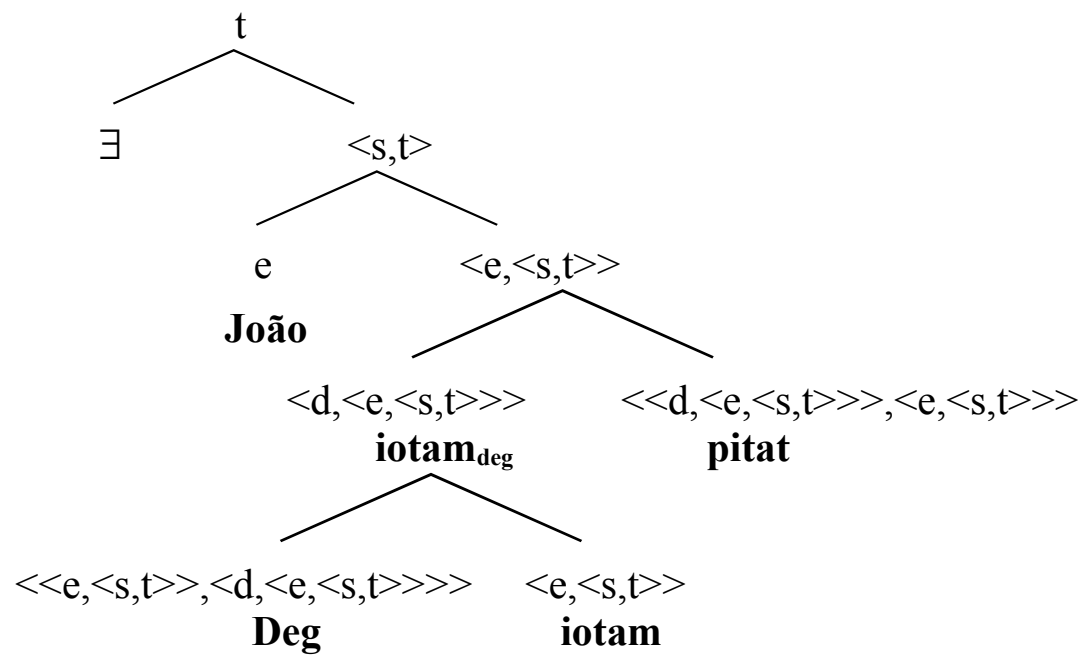

(269) Composição Semântica:

1. [[ iotam $\left.\left._{d e g}\right]\right]=[[$ Deg $]]([[$ iotam $]]) \quad$ Aplicação Funcional

$=[\lambda \mathrm{P} \lambda \mathrm{d} \lambda \mathrm{x} \lambda \mathrm{e} \cdot \mathrm{P}(\mathrm{x})(\mathrm{e}) \& \mu(\mathrm{e})=\mathrm{d}](\lambda \mathrm{x} \lambda \mathrm{e} \cdot \operatorname{chegar}(\mathrm{x})(\mathrm{e}))$

$=\lambda \mathrm{d} \lambda \mathrm{x} \lambda \mathrm{e}$. chegar $(\mathrm{x})(\mathrm{e}) \&$ “chegar" $(\mathrm{e})=\mathrm{d}$

2. [[ iotam pitat $]]=[[$ pitat $]]\left(\left[\left[\right.\right.\right.$ iotam $\left.\left.\left._{\text {deg }}\right]\right]\right) \quad$ Aplicação Funcional $=\left[\lambda \mathrm{G} \lambda \mathrm{x} \lambda \mathrm{e} . \exists \mathrm{d}\left[\mathrm{G}(\mathrm{d})(\mathrm{x})(\mathrm{e}) \& \mathrm{~d} \geq \mathrm{d}_{\mathrm{s}}\right]\right](\lambda \mathrm{d} \lambda \mathrm{x} \lambda \mathrm{e}$. chegar $(\mathrm{x})(\mathrm{e}) \&$ “chegar"(e) =d)

$=\lambda \mathrm{x} \lambda \mathrm{e} . \exists \mathrm{d}\left[\operatorname{chegar}(\mathrm{x})(\mathrm{e}) \&\right.$ "chegar" $(\mathrm{e})=\mathrm{d} \& \mathrm{~d}=\mathrm{d}_{\mathrm{s}}$

3. [[ João iotam pitat $]]_{\mathrm{s}, \downarrow}=[[$ iotam pitat $]]([[$ João $]]$ ) Aplicação Funcional

$=\left[\lambda x \lambda e . \exists d\left[\operatorname{chegar}(x)(e) \&\right.\right.$ “chegar" $\left.\left.(e)=d \& d=d_{s}\right]\right](J o a ̃ o)$

$=\lambda$ e. $\exists \mathrm{d}\left[\right.$ chegar $\left(\right.$ João)(e) \& “chegar”(e) $\left.=\mathrm{d} \& \mathrm{~d}=\mathrm{d}_{\mathrm{s}}\right]$

4. [[ João iotam pitat ] $]_{\mathrm{t}}=1$ sse $\exists \mathrm{e} \exists \mathrm{d}\left[\operatorname{chegar}(J o a ̃ o)(e) \&\right.$ “chegar”(e) $\left.=\mathrm{d} \& \mathrm{~d}=\mathrm{d}_{\mathrm{s}}\right]$

Em palavras: A sentença João iotam pitat é verdadeira se, e somente se, existe um evento e, e existe um grau d; o evento e é um evento de chegar de João e o evento e é mapeado na escala “chegar” em um grau d que é igual ao grau $\mathbf{d}_{\mathbf{s}}$ (que representa o grau máximo da escala, uma vez que se trata de uma escala fechada). 
(270) João i-pykyn-<a>-t pita-t.

João PART-chegar-ABS pita-ADV

'O João correu muito'

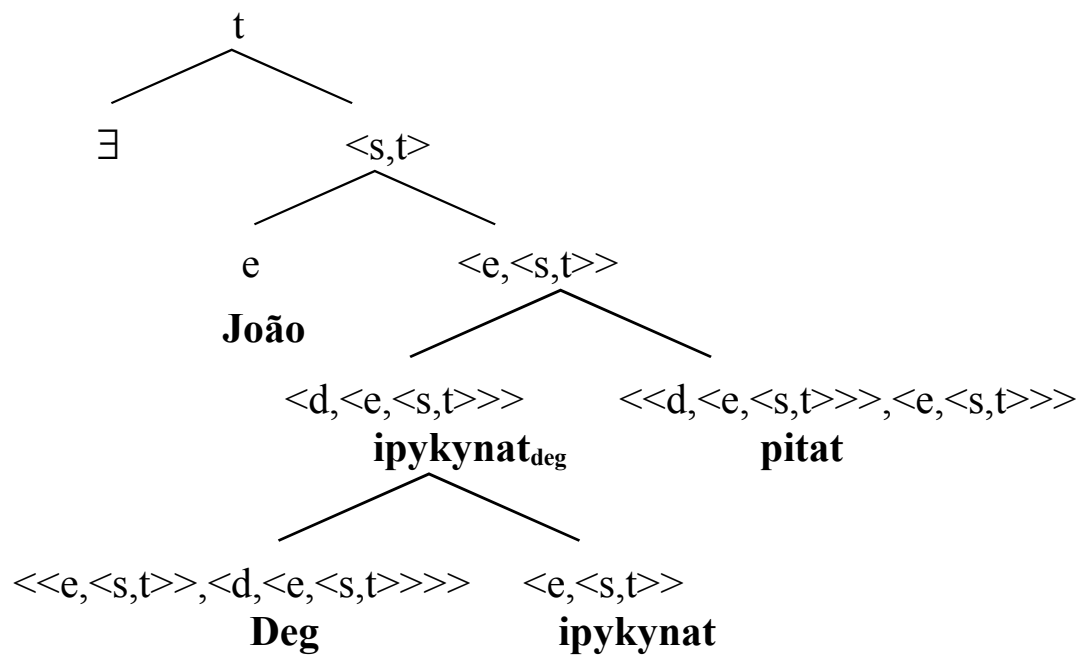

(272) Composição Semântica:

1. [[ ipykynat deg $\left._{]}\right]=[[\operatorname{Deg}]]([[$ ipykynat $]]) \quad$ Aplicação Funcional

$$
\begin{aligned}
& =[\lambda \mathrm{P} \lambda \mathrm{d} \lambda \mathrm{x} \lambda \mathrm{e} \cdot \mathrm{P}(\mathrm{x})(\mathrm{e}) \& \mu(\mathrm{e})=\mathrm{d}](\lambda \mathrm{x} \lambda \mathrm{e} \text {. correr })(\mathrm{e})) \\
& =\lambda \mathrm{d} \lambda \mathrm{x} \lambda \mathrm{e} . \text { correr }(\mathrm{x})(\mathrm{e}) \& \mu(\mathrm{e})=\mathrm{d}
\end{aligned}
$$

2. [[ ipykynat pitat ]] =[[ pitat ] $]\left(\left[\left[\right.\right.\right.$ ipykynat $\left.\left.\left._{d e g}\right]\right]\right) \quad$ Aplicação Funcional

$$
\begin{aligned}
& =\left[\lambda \mathrm{G} \lambda \mathrm{x} \lambda \mathrm{e} . \exists \mathrm{d}\left[\mathrm{G}(\mathrm{d})(\mathrm{x})(\mathrm{e}) \& \mathrm{~d} \geq \mathrm{d}_{\mathrm{s}}\right]\right](\lambda \mathrm{d} \lambda \mathrm{x} \lambda \mathrm{e} . \operatorname{correr}(\mathrm{x})(\mathrm{e}) \& \\
& \mu(\mathrm{e})=\mathrm{d}) \\
& =\lambda \mathrm{x} \lambda \mathrm{e} . \exists \mathrm{d}\left[\operatorname{correr}(\mathrm{x})(\mathrm{e}) \& \mu(\mathrm{e})=\mathrm{d} \& \mathrm{~d}>\mathrm{d}_{\mathrm{s}}\right.
\end{aligned}
$$

3. [[ João ipykynat pitat $]]_{<\mathrm{s}, \triangleright}=[[$ ipykynat pitat $]]([[$ João $]]$ ) Aplicação Funcional

$$
\begin{aligned}
& =\left[\lambda \mathrm{x} \lambda \mathrm{e} . \exists \mathrm{d}\left[\operatorname{correr}(\mathrm{x})(\mathrm{e}) \& \mu(\mathrm{e})=\mathrm{d} \& \mathrm{~d}>\mathrm{d}_{\mathrm{s}}\right]\right](\text { João }) \\
& =\lambda \mathrm{e} . \exists \mathrm{d}\left[\operatorname{correr}(\mathrm{João})(\mathrm{e}) \& \mu(\mathrm{e})=\mathrm{d} \& \mathrm{~d}>\mathrm{d}_{\mathrm{s}}\right]
\end{aligned}
$$

4. [[ João ipykynat pitat $]]_{\mathrm{t}}=1$ sse $\exists \mathrm{e} \exists \mathrm{d}\left[\operatorname{correr}(\mathrm{João})(\mathrm{e}) \& \mu(\mathrm{e})=\mathrm{d} \& \mathrm{~d}>\mathrm{d}_{\mathrm{s}}\right]$

Em palavras: A sentença João ipykynat pitat é verdadeira se, e somente se, existe um evento e, e existe um grau d; o evento e é um evento de correr de João e o evento e é mapeado em uma escala $\boldsymbol{\mu}$ em um grau $\mathbf{d}$ que é maior do que o grau $\mathbf{d}_{\mathbf{s}}$ (que representa o grau normal da escala, uma vez que se trata de uma escala aberta). $\boldsymbol{\mu}$ pode ser tempo decorrido, quantidade de 
ocorrências, distância, velocidade ou intensidade.

Esta seção mostrou a nossa proposta de análise para pitat quando modifica predicados verbais. A próxima seção trata da modificação de substantivos.

\subsection{Substantivos}

O objetivo desta seção é apresentar uma proposta para o uso de pita modificando substantivos. A tabela abaixo mostra o uso de pita com alguns substantivos. Sua distribuição é a de um adjetivo típico, depois do substantivo modificado. Embora em todas as sentenças pita tenha sido traduzido por 'verdadeiro', essa noção varia de acordo com o substantivo modificado, explicitada por meio das situações.

Tabela 49: Pita Modificando Substantivos

\begin{tabular}{|c|c|}
\hline Substantivo & Exemplo \\
\hline $\begin{array}{l}\text { taso } \\
\text { 'homem' }\end{array}$ & $\begin{array}{l}\text { [Taso pita] i-otam- } \varnothing \text {. } \\
\text { homem pita PART-chegar-ABS } \\
\text { 'O homem verdadeiro chegou' } \\
\text { Situação: um homem valoroso/caçador }\end{array}$ \\
\hline $\begin{array}{l}\text { jonso } \\
\text { 'mulher' }\end{array}$ & $\begin{array}{l}\text { [jonso pita] i-otam- } \varnothing \text {. } \\
\text { mulher pita PART-chegar-ABS } \\
\text { 'A mulher verdadeira chegou' } \\
\text { Situação: uma mulher valorosa/trabalhadora }\end{array}$ \\
\hline $\begin{array}{l}\text { gooj } \\
\text { 'carro' }\end{array}$ & $\begin{array}{l}\text { João i-amy-t } \quad \text { gooj pita]. } \\
\text { João PART-comprar-ABS carro pita } \\
\text { 'O João comprou um carro verdadeiro' } \\
\text { Situação: um carro original/ da loja/ um carro bom }\end{array}$ \\
\hline $\begin{array}{l}\text { kinda'o } \\
\text { 'fruta' }\end{array}$ & $\begin{array}{l}\text { João i-amy-t [kinda'o pita]. } \\
\text { João PART-comprar-ABS fruta pita } \\
\text { 'O João comprou uma fruta verdadeira' } \\
\text { Situação: uma fruta boa/ de primeira qualidade }\end{array}$ \\
\hline
\end{tabular}




\begin{tabular}{|c|c|}
\hline him & $\begin{array}{l}\text { João i-amy-t [him pita }] \text {. } \\
\text { João PART-comprar-ABS carne pita } \\
\text { 'O João comprou uma carne verdadeira' } \\
\text { Situação: uma carne boa/ de primeira qualidade }\end{array}$ \\
\hline $\begin{array}{l}\text { sypomp pykyp } \\
\text { 'óculos' }\end{array}$ & $\begin{array}{l}\text { João i-amy-t } \quad \text { sypomp.pykyp } \\
\text { João PART-comprar-ABS }\end{array}$ \\
\hline $\begin{array}{l}\text { celular } \\
\text { 'celular' }\end{array}$ & $\begin{array}{l}\text { João i-amy-t } \quad \text { celular pita]. } \\
\text { João PART-comprar-ABS celular pita } \\
\text { 'O João comprou um celular verdadeiro' } \\
\text { Situação: um celular original / em uma loja do shopping }\end{array}$ \\
\hline $\begin{array}{l}\text { ti'y } \\
\text { 'comida' }\end{array}$ & $\begin{array}{l}\text { João i-amy-t [ti'y pita]. } \\
\text { João PART-comprar-ABS comida pita } \\
\text { 'O João comprou comida verdadeira' } \\
\text { Situação: comida boa / de primeira qualidade }\end{array}$ \\
\hline $\begin{array}{l}\text { opi } \\
\text { 'brinco' }\end{array}$ & $\begin{array}{l}\text { João i-amy-t [opi pita]. } \\
\text { João PART-comprar-ABS brinco pita } \\
\text { 'O João comprou um brinco verdadeiro' } \\
\text { Situação: um brinco com material tradicional (sementes) }\end{array}$ \\
\hline $\begin{array}{l}\text { ombaky } \\
\text { 'onça' }\end{array}$ & $\begin{array}{l}\text { João i-so'ot- } \varnothing \quad \text { [ombaky pita]-ty. } \\
\text { João PART-ver-ABS onça pita-OBL } \\
\text { 'O João viu uma onça verdadeira' } \\
\text { Situação: onça de verdade }\end{array}$ \\
\hline
\end{tabular}

Na tabela acima, a tradução 'verdadeiro' foi mantida porque ela de alguma forma uniformiza as noções expressas. O contrário de pita nessas sentenças é algo do tipo 'falso' ou 'de brinquedo'. Essas duas noções são expressas pelos adjetivos pymbyra e õwõrã respectivamente que são apresentados nas tabelas abaixo. Interessantemente, pymbyra também parece ser um modificador grau, que significa 'pouco' na língua. O constraste apresentado ajuda a entender o significado de pita. 
Tabela 50: Substantivos Modificados por Pymbyra

\begin{tabular}{|c|c|}
\hline Substantivo & Exemplo \\
\hline $\begin{array}{l}\text { taso } \\
\text { 'homem' }\end{array}$ & $\begin{array}{l}\text { [Taso pymbyra] i-otam- } \varnothing \text {. } \\
\text { homem falso PART-chegar-ABS } \\
\text { 'Um homem que não é de verdade chegou' } \\
\text { Situação: um homem que não trabalha / que não está preparado para } \\
\text { ser homem }\end{array}$ \\
\hline $\begin{array}{l}\text { jonso } \\
\text { 'mulher' }\end{array}$ & $\begin{array}{l}\text { [jonso pymbyra] i-otam- } \varnothing \text {. } \\
\text { mulher falso PART-chegar-ABS } \\
\text { 'Uma mulherque não é de verdade chegou' } \\
\text { Situação: uma mulher preguiçosa / que não é valorosa }\end{array}$ \\
\hline $\begin{array}{l}\text { gooj } \\
\text { 'carro' }\end{array}$ & $\begin{array}{l}\text { João i-amy-t } \quad \text { [gooj pymbyra]. } \\
\text { João PART-comprar-ABS carro falso } \\
\text { 'O João comprou um carro que não é de verdade' } \\
\text { Situação: um carro pirata / que não é original }\end{array}$ \\
\hline $\begin{array}{l}\text { 'kinda'o } \\
\text { 'fruta' }\end{array}$ & $\begin{array}{l}\text { João i-amy-t [kinda'o pymbyra]. } \\
\text { João PART-comprar-ABS fruta falso } \\
\text { 'O João comprou uma fruta que não é de verdade' } \\
\text { Situação: uma fruta ruim / manga }\end{array}$ \\
\hline $\begin{array}{l}\text { him } \\
\text { 'carne' }\end{array}$ & $\begin{array}{l}\text { João i-amy-t [him pymbyra }] \text {. } \\
\text { João PART-comprar-ABS carne falso } \\
\text { 'O João comprou uma carne que não é de verdade' } \\
\text { Situação: uma carne de segunda }\end{array}$ \\
\hline $\begin{array}{l}\text { sypomp pykyp } \\
\text { 'óculos' }\end{array}$ & 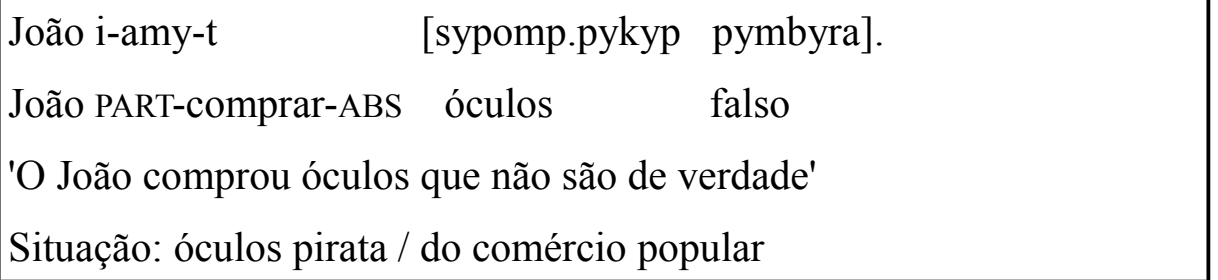 \\
\hline $\begin{array}{l}\text { celular } \\
\text { 'celular' }\end{array}$ & $\begin{array}{l}\text { João i-amy-t } \quad \text { celular pymbyra]. } \\
\text { João PART-comprar-ABS celular falso } \\
\text { 'O João comprou um celular que não é de verdade' } \\
\text { Situação: celular pirata / do comércio popular' }\end{array}$ \\
\hline
\end{tabular}




\begin{tabular}{|c|c|}
\hline $\begin{array}{l}t i ' y \\
\text { 'comida' }\end{array}$ & $\begin{array}{l}\text { João i-amy-t [ti'y pymbyra]. } \\
\text { João PART-comprar-ABS comida falso } \\
\text { 'O João comprou comida que não é de verdade' } \\
\text { Situação: comida de qualidade inferior' }\end{array}$ \\
\hline $\begin{array}{l}\text { opi } \\
\text { 'brinco' }\end{array}$ & $\begin{array}{l}\text { João i-amy-t [opi pymbyra]. } \\
\text { João PART-comprar-ABS brinco falso } \\
\text { 'O João comprou um brinco que não é de verdade' } \\
\text { Situação: brinco de material ruim / comprado no comércio popular' }\end{array}$ \\
\hline $\begin{array}{l}\text { ombaky } \\
\text { 'onça' }\end{array}$ & $\begin{array}{l}\text { João i-so'ot- } \varnothing \quad \text { [ombaky pymbyra]-ty. } \\
\text { João PART-ver-ABS onça falso-OBL } \\
\text { 'O João viu uma onça que não é de verdade' } \\
\text { Situação: um gato / um animal que parece onça, mas não é onça' }\end{array}$ \\
\hline
\end{tabular}

Tabela 51: Substantivos Modificados por $\tilde{O}$ wõrã

\begin{tabular}{|c|c|}
\hline Substantivo & Exemplo \\
\hline taso & [Taso õwõrã $]$ i-otam- $\varnothing$. \\
\hline \multirow[t]{3}{*}{ 'homem' } & homem de.brinquedo PART-chegar-ABS \\
\hline & 'Um homem de brincadeira chegou' \\
\hline & Situação: alguma criança se passando por homem em uma brincadeira \\
\hline jonso & [jonso õwõrã] $\quad$ i-otam- $\varnothing$. \\
\hline \multirow[t]{3}{*}{ 'mulher' } & mulher de.brinquedo PART-chegar-ABS \\
\hline & 'Uma mulher de brincadeira chegou' \\
\hline & Situação: alguma criança se passando por mulher em uma brincadeira \\
\hline gooj & João i-amy-t $\quad$ [gooj õwõrã]. \\
\hline \multirow[t]{2}{*}{ 'carro' } & João PART-comprar-ABS carro de.brinquedo \\
\hline & 'O João comprou um carrinho de brinquedo' \\
\hline$k i n d a^{\prime} o$ & João i-amy-t $\quad$ [kinda'o õwõrã $]$. \\
\hline \multirow[t]{3}{*}{ 'fruta' } & João PART-comprar-ABS fruta de.brinquedo \\
\hline & 'O João comprou uma fruta de brincadeira' \\
\hline & de má qualidade' \\
\hline
\end{tabular}




\begin{tabular}{|c|c|}
\hline $\begin{array}{l}\text { 'him } \\
\text { 'carne' }\end{array}$ & $\begin{array}{l}\text { João i-amy-t [him õwõrã]. } \\
\text { João PART-comprar-ABS carne de.brinquedo } \\
\text { 'O João comprou uma carne de segunda' }\end{array}$ \\
\hline $\begin{array}{l}\text { sypomp pykyp } \\
\text { 'óculos' }\end{array}$ & \begin{tabular}{|lcl} 
João i-amy-t $\quad$ [sypomp.pykyp & õwõrã]. \\
João PART-comprar-ABS & óculos & de.brinquedo \\
'O João comprou óculos de brinquedo' &
\end{tabular} \\
\hline $\begin{array}{l}\text { celular } \\
\text { 'celular' }\end{array}$ & $\begin{array}{l}\text { João i-amy-t } \quad \text { celular õwõrã }] \\
\text { João PART-comprar-ABS celular de.brinquedo } \\
\text { 'O João comprou um celular de brinquedo' }\end{array}$ \\
\hline $\begin{array}{l}t i{ }^{\prime} y \\
\text { 'comida' }\end{array}$ & 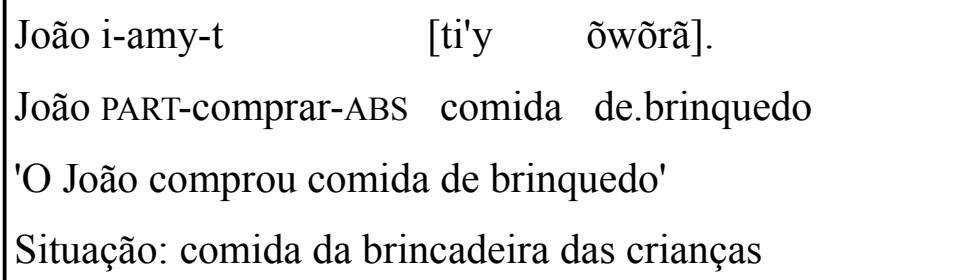 \\
\hline $\begin{array}{l}\text { opi } \\
\text { 'brinco' }\end{array}$ & 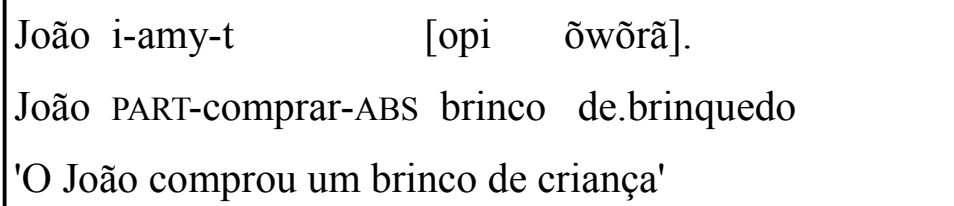 \\
\hline $\begin{array}{l}\text { ombaky } \\
\text { 'onça' }\end{array}$ & $\begin{array}{l}\text { João i-so'ot- } \varnothing \quad \text { [ombaky õwõrã]-ty. } \\
\text { João PART-ver-ABS onça de.brinquedo-OBL } \\
\text { 'O João viu uma onça de brinquedo' } \\
\text { Situação: uma onça de pelúcia }\end{array}$ \\
\hline
\end{tabular}

As tabelas acima mostraram que, quando pita modifica substantivos, ele significa algo do tipo 'verdadeiro' por oposição a 'falso', mas também tem uma noção de valor, como nos casos de 'homem valoroso' e 'comida de primeira'. Para analisar esse uso vamos adotar a análise de Masià (2013) para os adjetivos de veracidade em espanhol. Segundo a autora, o adjetivo verdadero 'verdadeiro' na sentença abaixo força o substantivo a ser interpretado em seu sentido estrito, o substantivo tem que ser interpretado de modo preciso.

(273) Paloma es una verdadera artista.

'Paloma é uma verdadeira artista' 
Masià (2013) adota a proposta de Morzycki (2011) de que substantivos podem estar associados a uma escala de precisão. Segundo Masià (2013), o verdadero do espanhol é um modificador dessa escala que seleciona o grau máximo de precisão. A implementação formal da proposta de Morzycki (2011) começa com o estabelecimento de uma relação de aproximação $\approx$ que fornece o parâmetro de similaridade, definida abaixo.

(274) $\alpha \approx_{\mathrm{d}, \mathrm{C}} \beta$ se e somente se, dada a ordem estabelecida pelo contexto $\mathbf{C}, \boldsymbol{\alpha}$ se parece $\operatorname{com} \boldsymbol{\beta}$ em (pelo menos) um grau $\mathbf{d}$ e $\boldsymbol{\alpha}$ e $\boldsymbol{\beta}$ são do mesmo tipo.

A escala de precisão é fornecida por meio das alternativas geradas pela relação de aproximação $\approx$ (dada por um grau de semelhança) e que podem, a princípio ser intercambiáveis. Quanto mais se aumenta o grau de precisão, menor é o número de alternativas possíveis. E quando o grau de precisão é máximo, o substantivo precisa ser interpretado de modo preciso. As denotações abaixo apresentam um exemplo da proposta. (275a) representa a denotação do substantivo artista segundo um grau de precisão e ordenado por um contexto C. O resultado é um conjunto de predicados de tipo $<$ e,t $>$ que tem uma relação de similaridade com o predicado artista. O conjunto descrito em (275b) representaria o grau de precisão 0.8 da escala. O exemplo (275c) apresenta um grau de precisão maior, por exemplo, 0.9, logo seu conjunto de alternativas é menor. Quando o grau de precisão atinge seu valor máximo, o valor 1, a denotação é o cunjunto unitário contendo apenas o predicado considerado. (275e) ilustra que, quando o grau de precisão é zero, a denotação do predicado é todo o domínio de tipo $<\mathrm{e}, \mathrm{t}>$.

(275) a. $\quad[[\operatorname{artista}]]^{\mathrm{d}, \mathrm{C}}=\left\{\mathrm{f}_{<\mathrm{e},}: \mathrm{f} \approx_{\mathrm{d}, \mathrm{C}} \operatorname{artista}\right\}$

b. $\quad[[\text { artista }]]^{08, \mathrm{C}}=\{$ artista, criador, autor, artesão, desenhista $\}$

c. $\quad[[\text { artista }]]^{0.9, \mathrm{C}}=$ artista, criador, autor $\}$

d. $\quad[[\operatorname{artista}]]^{1, \mathrm{C}}=\{\operatorname{artista}\}$

e. $\quad[[\operatorname{artista}]]^{0, \mathrm{C}}=\mathrm{D}_{<\mathrm{e}, \triangleright}$

Para deixar o grau de precisão disponível para a composição semântica, Morzycki (2011) e Masià (2013) adotam uma Semântica Intensional que mapeia as proposições em suas proposições alternativas. Para manter a uniformidade da nossa proposta, vamos adotar a 
proposta dos autores baseada na escala de precisão, mas vamos adaptá-la à Semântica Escalar que estamos adotando neste trabalho. Para tal, vamos assumir que todos os substantivos podem ter uma versão graduável e ser mapeados em uma escala de precisão. Desse modo, um substantivo como homem, além de ter sua entrada lexical tradicional (276a), pode ter uma versão graduável que mapeia o predicado na escala de precisão (276b).

$$
\begin{aligned}
& \text { a. } \quad[[\text { homem }]]=\lambda \mathrm{x} . \operatorname{homem}(\mathrm{x}) \\
& \text { b. }\left[\left[\text { homem }_{\text {deg }}\right]\right]=\lambda \mathrm{d} \lambda \mathrm{x} . \operatorname{homem}(\mathrm{x}) \& \text { "precisão" }(\mathrm{x})=\mathrm{d}
\end{aligned}
$$

A escala de precisão nesse caso também deve ser adaptada porque a seleção de um taso pita 'homem verdadeiro' se dá no conjunto dos homens. Conforme as tabelas acima mostraram, um taso pita é um homem que se destaca em um conjunto de homens, por ser um homem valoroso, com qualidades, e não em um conjunto de predicados alternativos que podem substituir o predicado homem, como o exemplo com artista fornecido por Masià (2013). Estamos considerando, então, que a escala de precisão é fornecida com o ordenamento do conjunto de homens em graus de precisão, que vão do mais preciso ao menos preciso. A versão graduável do predicado nominal pode ser obtida por meio de uma função de

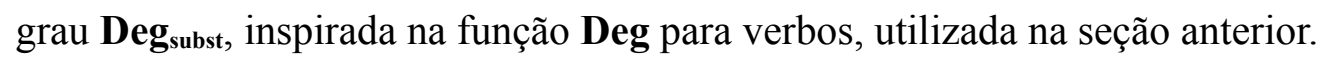

$$
\left[\left[\text { Deg }_{\text {subst }}\right]\right]=\lambda \mathrm{P}_{<\mathrm{e}, \downarrow} \lambda \mathrm{d} \lambda \mathrm{x} . \mathrm{P}(\mathrm{x}) \& \text { "precisão" } \mathrm{P}(\mathrm{x})=\mathrm{d}
$$

A escala "precisãop" é a escala de precisão associada ao predicado $\mathbf{P}$. Na proposta de Morzycki (2011), a escala de precisão vai de 0 a 1. Logo, trata-se de uma escala fechada nos graus mínimos e máximo (semelhante à escala de ocupação dos adjetivos cheio e vazio). Nossa proposta é a de que a mesma versão de pita que modifica adjetivos modifique também substantivos em sua versão graduável com escala de precisão. A denotação de pita é repetida abaixo:

$$
[[\text { pita }]]=\lambda \mathrm{G} \lambda \mathrm{x} . \exists \mathrm{d}\left[\mathrm{G}(\mathrm{d})(\mathrm{x}) \& \mathrm{~d} \geq \mathrm{d}_{\mathrm{s}}\right]
$$

Conforme apresentado nas seções anteriores, o grau $\mathbf{d}_{\mathbf{s}}$ representa o grau normal se pita estiver modificando um predicado de escala aberta, e o grau máximo se estiver 
modificando um predicado de escala fechada. Como a escala de precisão é uma escala fechada, pita seleciona o grau máximo de precisão. Nesse sentido, seu significado é de que o substantivo modificado deve ser interpretado na sua forma mais precisa, o mais próximo do ideal. Daí a sua interpretação de verdadeiro. O que vai significar ser verdadeiro para cada tipo de substantivo é atribuído pelo parâmetro de comparação que é representado também por $\mathbf{d}_{\mathbf{s}}$. O parâmetro contextual seleciona em relação a que característica o substantivo que representa o sentido estrito deve ser selecionado. Conforme a tabela 49 mostrou, essa característica está normalmente associada à qualidade, mas ela pode também ser representada pela precisão da aplicação do predicado, como no caso de ombaky 'onça'.

Apresentamos abaixo um exemplo de composição semântica com pita modificando substantivos.

(279) [Taso pita] i-otam-Ø.

homem pita PART-chegar-ABS

'O homem verdadeiro chegou'

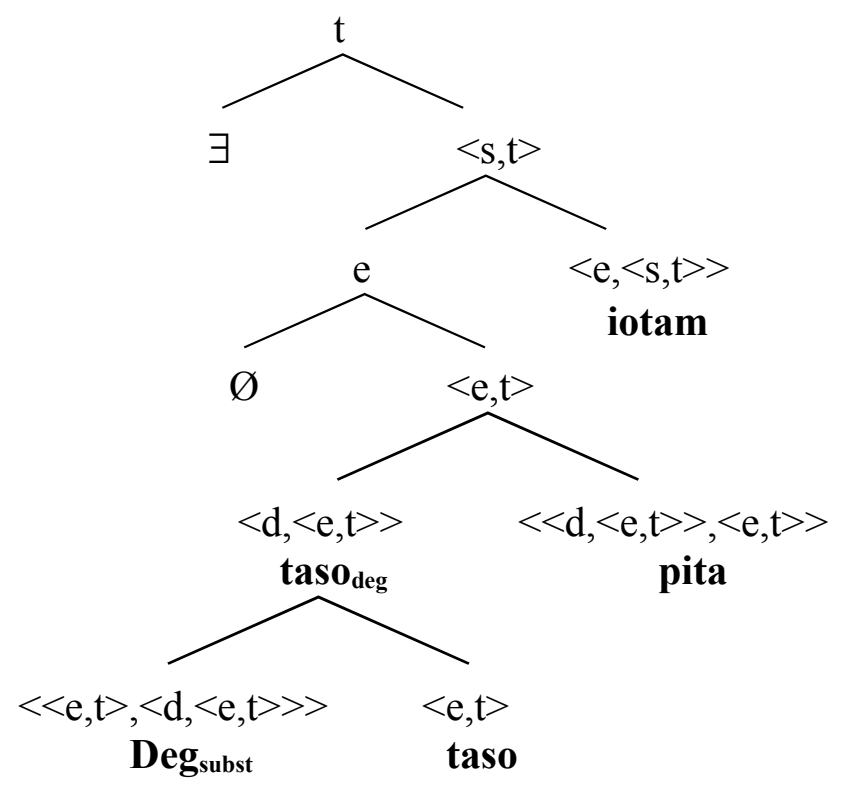


1. [[ taso $\left.\left._{\text {deg }}\right]\right]=\left[\left[\right.\right.$ Deg $\left.\left._{\text {subst }}\right]\right]([[$ taso $]])$

Aplicação Funcional

$=\left[\lambda \mathrm{P}_{<\mathrm{e}, \triangleright} \lambda \mathrm{d} \lambda \mathrm{x} . \mathrm{P}(\mathrm{x}) \&\right.$ "precisão $\left.{ }_{\mathrm{P}}(\mathrm{x})=\mathrm{d}\right](\lambda \mathrm{x}$. homem $(\mathrm{x}))$

$=\lambda \mathrm{d} \lambda \mathrm{x} \cdot \operatorname{homem}(\mathrm{x}) \&$ "precisão, $(\mathrm{x})=\mathrm{d}$

2. $[[\text { taso pita }]]_{<\mathrm{e}, \triangleright}=[[$ pita $]]([[$ taso $]])$

Aplicação Funcional

$=\left[\lambda \mathrm{G} \lambda \mathrm{x} . \exists \mathrm{d}\left[\mathrm{G}(\mathrm{d})(\mathrm{x}) \& \mathrm{~d} \geq \mathrm{d}_{\mathrm{s}}\right]\right]\left(\lambda \mathrm{d} \lambda \mathrm{x} . \operatorname{homem}(\mathrm{x}) \&\right.$ "precisão $\left.\mathrm{P}_{\mathrm{P}}(\mathrm{x})=\mathrm{d}\right)$

$=\lambda \mathrm{x} . \exists \mathrm{d}\left[\operatorname{homem}(\mathrm{x}) \&\right.$ "precisão" $\left.(\mathrm{x})=\mathrm{d} \& \mathrm{~d}=\mathrm{d}_{\mathrm{s}}\right]$

3. [[ taso pita def $\left.\left._{]}\right]\right]_{\mathrm{e}}=[[\varnothing]]([[$ taso pita $]])$

Aplicação Funcional

$=\lambda \mathrm{f}$. o único $\mathrm{x}$ no contexto tal que $\mathrm{f}(\mathrm{x})=1(\lambda \mathrm{x} . \exists \mathrm{d}[\mathrm{homem}(\mathrm{x}) \&$ "precisãop" (x) $\left.=\mathrm{d} \& \mathrm{~d}=\mathrm{d}_{\mathrm{s}}\right]$ )

$=\mathrm{o}$ único x no contexto tal que $\exists \mathrm{d}[\operatorname{homem}(\mathrm{x}) \&$ "precisãop" $(\mathrm{x})=\mathrm{d}$ $\left.\& \mathrm{~d}=\mathrm{d}_{\mathrm{s}}\right]$

4. [[ taso pita iotam $]]_{\mathrm{s}_{\mathrm{s}, \triangleright}}=[[$ iotam $]]\left(\left[\left[\right.\right.\right.$ taso $\left.\left.\left._{\text {pita }}\right]\right]\right) \quad$ Aplicação Funcional

$=\lambda \mathrm{x} \lambda \mathrm{e}$. chegar(x)(e) (o único $\mathrm{x}$ no contexto tal que $\exists \mathrm{d}[\mathrm{homem}(\mathrm{x}) \&$ "precisão," (x) $\left.\left.=\mathrm{d} \& \mathrm{~d}=\mathrm{d}_{\mathrm{s}}\right]\right)$

$=\lambda$ e. chegar(o único x no contexto tal que $\exists \mathrm{d}[$ homem(x) \& "precisãop" (x) $\left.\left.=\mathrm{d} \& \mathrm{~d}=\mathrm{d}_{\mathrm{s}}\right]\right)(\mathrm{e})$

5. [[ taso pita iotam ] ] $]_{\mathrm{t}}=1$ sse $\exists \mathrm{e} \exists \mathrm{d}$ [ chegar(o único $\mathrm{x}$ no contexto tal que [homem(x) \& "precisãop" (x) $\left.\left.\left.=\mathrm{d} \& \mathrm{~d}=\mathrm{d}_{\mathrm{s}}\right]\right]\right)(\mathrm{e})$

Em palavras: A sentença Taso pita iotam é verdadeira se, e somente se, existe um evento e, e existe um grau $\mathbf{d}$; o evento e é um evento de chegar do único $\mathbf{x}$ no contexto tal que $\mathbf{x}$ é homem e $\mathbf{x}$ é mapeado na escala de precisão em um grau $\mathbf{d}$ que é igual ao grau $\mathbf{d}_{\mathbf{s}}$ (que representa o grau máximo da escala, uma vez que se trata de uma escala fechada).

Esta seção apresentou uma análise para o uso de pita modificando substantivos. A próxima seção apresenta uma análise unificada para pita(t) em todos os domínios. 
A seção 3 mostrou a distribuição de pita(t) com adjetivos, verbos e substantivos. Vimos que, a despeito da diferença no significado, pita(t) pode ser analisado como um modificador de grau em todos os domínios. Isso quer dizer que ele sempre está procurando um predicado com um argumento de grau de tipo d. No entanto, a proposta apresentou duas versões de pita(t), uma modificando substantivos e adjetivos e outra modificando verbos.

A entrada lexical de pita modificando substantivos e adjetivos é a seguinte:

$$
[[\text { pita }]]=\lambda \mathrm{G}_{<\mathrm{d},<\mathrm{e}, \triangleright>} \lambda \mathrm{x}_{\mathrm{e}} . \exists \mathrm{d}\left[\mathrm{G}(\mathrm{d})(\mathrm{x}) \& \mathrm{~d} \geq \mathrm{d}_{\mathrm{s}}\right]
$$

Já pitat modificando predicados verbais tem a seguinte denotação:

$$
[[\text { pitat }]]=\lambda \mathrm{G}_{<\mathrm{d},<\mathrm{e},<\mathrm{s}, \gg \gg>} \lambda \mathrm{x}_{\mathrm{e}} \lambda \mathrm{e}_{\mathrm{s} .} \exists \mathrm{d}\left[\mathrm{G}(\mathrm{d})(\mathrm{x})(\mathrm{e}) \& \mathrm{~d} \geq \mathrm{d}_{\mathrm{s}}\right]
$$

Para simplificar, vamos chamar o predicado graduável de tipo $<\mathrm{d},<\mathrm{e}, \mathrm{t}>>$ de $\mathbf{G}_{\mathbf{A}}$ e o predicado graduável de tipo $<\mathrm{d},<\mathrm{e},<\mathrm{s}, \mathrm{t}>>>\mathrm{de}_{\mathrm{V}}$. Assim, as fórmulas podem ser simplificadas da seguinte forma:

$$
\begin{array}{ll}
\text { a. } & {[[\text { pita }]]=\lambda \mathrm{G}_{\mathrm{A}} \lambda \mathrm{x}_{\mathrm{e}} \cdot \exists \mathrm{d}\left[\mathrm{G}_{\mathrm{A}}(\mathrm{d})(\mathrm{x}) \& \mathrm{~d} \geq \mathrm{d}_{\mathrm{s}}\right]} \\
\text { b. } & {[[\text { pitat }]]=\lambda \mathrm{G}_{\mathrm{v}} \lambda \mathrm{x}_{\mathrm{e}} \lambda \mathrm{e}_{\mathrm{s}} \cdot \exists \mathrm{d}\left[\mathrm{G}_{\mathrm{v}}(\mathrm{d})(\mathrm{x})(\mathrm{e}) \& \mathrm{~d} \geq \mathrm{d}_{\mathrm{s}}\right]}
\end{array}
$$

$\mathrm{O}$ que as denotações acima mostram é que o significado de pita(t) é basicamente o mesmo em todos os domínios. Em ambas as denotações, a contribuição semântica de pita(t) é a de que o grau $\mathbf{d}$ é maior ou igual ao grau $\mathbf{d}_{\text {s. }}$ O que muda em cada caso é o tipo de de predicado graduável G que o modificador seleciona. Pita seleciona predicados de tipo $<\mathrm{d},<\mathrm{e}, \mathrm{t}>>$ e pitat seleciona predicados de tipo $<\mathrm{d},<\mathrm{e},<\mathrm{s}, \mathrm{t}>>>$. Logo, a única diferença está na inserção de um tipo s de eventos na denotação de pitat.

Como vimos, sintaticamente, o sufixo $\{-t\}$ indica que o modificador é um adjunto do predicado verbal. Semanticamente, o que esse sufixo faz é inserir o tipo $\mathbf{s}$ de eventos na denotação do advérbio. Dessa forma, o sufixo tem a seguinte entrada lexical: 
Na denotação acima, $\mathbf{M}$ representa o modificador pita de tipo $<<\mathrm{d},<\mathrm{e}, \mathrm{t}>>,<\mathrm{e}, \mathrm{t}>>$. Assim, nossa proposta é de que há um modificador pita do qual pitat é derivado. A composição abaixo mostra como pitat é corretamente derivado de pita por meio do sufixo $\{-t\}$.

1. $[[-t]]=\lambda M \lambda \mathrm{G}_{\mathrm{v}} \lambda \mathrm{x}_{1} \lambda \mathrm{e}_{1} \cdot \mathrm{M}\left(\lambda \mathrm{d}_{1} \lambda \mathrm{x}_{2} \cdot \mathrm{G}_{\mathrm{v}}\left(\mathrm{d}_{1}\right)\left(\mathrm{x}_{2}\right)\left(\mathrm{e}_{1}\right)\right)\left(\mathrm{x}_{1}\right)$

Léxico

2. $[[$ pita $]]=\lambda \mathrm{G}_{\mathrm{A}} \lambda \mathrm{x}_{3} \cdot \exists \mathrm{d}_{2}\left[\mathrm{G}_{\mathrm{A}}\left(\mathrm{d}_{2}\right)\left(\mathrm{x}_{3}\right) \& \mathrm{~d}_{2} \geq \mathrm{d}_{\mathrm{s}}\right]$

Léxico
3. $[[$ pitat $]]=[[-t]]([[$ pita $]])$
Aplicação Funcional
$=\left[\lambda \mathrm{M} \lambda \mathrm{G}_{\mathrm{v}} \lambda \mathrm{x}_{1} \lambda \mathrm{e}_{1} \cdot \mathrm{M}\left(\lambda \mathrm{d}_{1} \lambda \mathrm{x}_{2} \cdot \mathrm{G}_{\mathrm{v}}\left(\mathrm{d}_{1}\right)\left(\mathrm{x}_{2}\right)\left(\mathrm{e}_{1}\right)\right)\left(\mathrm{x}_{1}\right)\right]\left(\lambda \mathrm{G}_{\mathrm{A}} \lambda \mathrm{x}_{3} \cdot \exists \mathrm{d}_{2}\left[\mathrm{G}_{\mathrm{A}}\left(\mathrm{d}_{2}\right)\left(\mathrm{x}_{3}\right) \& \mathrm{~d}_{2} \geq \mathrm{d}_{\mathrm{s}}\right]\right)$
$=\lambda \mathrm{G}_{\mathrm{v}} \lambda \mathrm{x}_{1} \lambda \mathrm{e}_{1} \cdot\left[\lambda \mathrm{G}_{\mathrm{A}} \lambda \mathrm{x}_{3} . \exists \mathrm{d}_{2}\left[\mathrm{G}_{\mathrm{A}}\left(\mathrm{d}_{2}\right)\left(\mathrm{x}_{3}\right) \& \mathrm{~d}_{2} \geq \mathrm{d}_{\mathrm{s}}\right]\right]\left(\lambda \mathrm{d}_{1} \lambda \mathrm{x}_{2} \cdot \mathrm{G}_{\mathrm{v}}\left(\mathrm{d}_{1}\right)\left(\mathrm{x}_{2}\right)\left(\mathrm{e}_{1}\right)\right)\left(\mathrm{x}_{1}\right)$
$=\lambda \mathrm{G}_{\mathrm{v}} \lambda \mathrm{x}_{1} \lambda \mathrm{e}_{1}\left[\lambda \mathrm{x}_{3} \cdot \exists \mathrm{d}_{2}\left[\lambda \mathrm{d}_{1} \lambda \mathrm{x}_{2} \cdot \mathrm{G}_{\mathrm{v}}\left(\mathrm{d}_{1}\right)\left(\mathrm{x}_{2}\right)\left(\mathrm{e}_{1}\right)\right]\left(\mathrm{d}_{2}\right)\left(\mathrm{x}_{3}\right) \& \mathrm{~d}_{2} \geq \mathrm{d}_{\mathrm{s}}\right]\left(\mathrm{x}_{1}\right)$
$=\lambda \mathrm{G}_{\mathrm{v}} \lambda \mathrm{x}_{1} \lambda \mathrm{e}_{1}\left[\lambda \mathrm{x}_{3} \cdot \exists \mathrm{d}_{2} \mathrm{G}_{\mathrm{v}}\left(\mathrm{d}_{2}\right)\left(\mathrm{x}_{3}\right)\left(\mathrm{e}_{1}\right) \& \mathrm{~d}_{2} \geq \mathrm{d}_{\mathrm{s}}\right]\left(\mathrm{x}_{1}\right)$
$=\lambda \mathrm{G}_{\mathrm{v}} \lambda \mathrm{x}_{1} \lambda \mathrm{e}_{1} \cdot \exists \mathrm{d}_{2}\left[\mathrm{G}_{\mathrm{v}}\left(\mathrm{d}_{2}\right)\left(\mathrm{x}_{1}\right)\left(\mathrm{e}_{1}\right) \& \mathrm{~d}_{2} \geq \mathrm{d}_{\mathrm{s}}\right]$

Uma vez apresentada nossa proposta unificada para pita(t), vamos explorar algumas consequências da proposta adotada nesta tese.

${ }^{100}$ Por qustão de clareza, estamos numerando as variáveis para diferenciá-las. 
O objetivo desta seção é discutir algumas consequências da proposta apresentada neste capítulo. A primeira consequência diz respeito à diferença entre a leitura iterativa e a leitura de grau, conforme discutidas no trabalho de Doetjes (2007) apresentado no capítulo 2. Voltando aos dados do Karitiana, observa-se que, com a indeterminação das escalas proposta na seção anterior, é possível explicar a leitura iterativa como parte da modificação de grau, sem a necessidade de uma regra de mudança do predicado de massivo para contável, como na proposta da autora. A sentença abaixo com o verbo pykyn 'correr' é vaga em relação às escalas a ela associadas. A leitura iterativa é apenas uma dessas escalas (a escala de ocorrêncoas disponível graças à natureza contável do predicado).

(287) Inácio i-pykyn-<a>-t pita-t.

Inácio PART-correr-ABS pita-ADV

'O Inácio correu muito'

(288) Situações em que a sentença (287) seria verdadeira:

'O Inácio correu por muito tempo'

'O Inácio correu em alta velocidade'

'O Inácio correu uma longa distância'

'O Inácio correu muitas vezes'

'O Inácio correu uma vez, com muita intensidade, fazendo muito esforço'

Logo, ao contrário da proposta de Doetjes (2007), a iteratividade é considerada como um subtipo da modificação de grau e não uma operação que compete com ela. Na realidade, na proposta assumida neste trabalho, não existe uma interpretação de grau. Existe uma modificação de grau (ou uma operação de grau) e há interpretações de iteratividade e intensidade que são geradas através dessa operação.

A segunda consequência da proposta deriva da hipótese de que construções envolvendo adjetivos graduáveis têm escalas lexicais, enquanto que construções envolvendo verbos atélicos têm escalas contextuais. Se essa assunção estiver correta, espera-se que a modificação de grau de adjetivos seja menos complexa que a de verbos. A aplicação da 
modificação de grau como em muito bonito é uma operação direta na escala lexicalizada de beleza. A modificação de grau de um verbo, por outro lado, envolve alguns passos adicionais. Uma vez que as escalas não são lexicalizadas nos verbos, para uma modificação do tipo correr muito ser interpretada, é preciso que a escala apropriada do verbo seja escolhida para que a construção seja avaliada. Formalmente, é preciso que o verbo passe por uma função Deg para ganhar um argumento de grau e uma variável de escalas.

Exatamente nessa distinção pode-se encontrar um esboço de explicação para a distribuição de very e a lot do inglês e de très e beaucoup em francês. Conforme descrito anteriormente, very e très se aplicam a adjetivos, e a lot e beaucoup se aplicam a sintagmas verbais. Very e très poderiam ser considerados como modificadores que se aplicam apenas a predicados com graus lexicalizados, enquanto que a lot e beaucoup forçariam o predicado a ter um argumento de grau (via Deg) para serem aplicados.

Essa mesma hipótese foi explorada em trabalho manuscrito intitulado A Aquisição de um Modificador de Grau em Português Brasileiro (Sanchez-Mendes, manuscrito). O objetivo do trabalho foi investigar preliminarmente a aquisição do modificador de grau muito em português brasileiro. Com base no pressuposto de que a modificação de grau de adjetivos é menos complexa que a de verbos e de que a aquisição da linguagem obedece a princípios de economia, partiu-se da hipótese de que as crianças utilizariam primeiramente a modificação de grau com adjetivos antes da modificação de grau com verbos.

Os dados coletados por meio de metodologia longitudinal coletados durante o período de aquisição de duas crianças adquirindo português brasileiro confirmam a hipótese. Eles mostram que as crianças produzem primeiramente sentenças com muito + adjetivo, e posteriormente dados com muito + verbo. A tabela abaixo mostra a porcentagem de ocorrência em cada estrutura nos dados de uma das crianças (Criança $L$ ).

Tabela 52: Dados com muito + adjetivo e muito + verbo (Criança $L)$

\begin{tabular}{|c|c|c|c|c|}
\hline Idade & muito + adjetivo & muito + verbo & Total & $\begin{array}{c}\text { Porcentagem de muito }+ \\
\text { adjetivo }\end{array}$ \\
\hline 2 anos & 16 & 0 & 16 & $100,00 \%$ \\
\hline 3 anos & 30 & 6 & 36 & $83,00 \%$ \\
\hline 4 anos & 66 & 5 & 71 & $93,00 \%$ \\
\hline 5 anos & 35 & 7 & 42 & $83,00 \%$ \\
\hline
\end{tabular}


Nos dados acima, é possível observar que a porcentagem da ocorrência de muito + adjetivo é maior que a ocorrência de muito + verbo em todas as idades, embora a diferença vá diminuindo com o crescimento da criança. Além disso, aos dois anos, a criança $L$ já produzia construções como muito + adjetivo, como os apresentados abaixo, mas ainda não aparecem sentenças com muito + verbo.

$\begin{array}{lll}\text { (289) } & \text { a. } & \text { Que muito difícil } \\ \text { b. } & \text { É muito ruim. } \\ \text { c. } & \text { Muito legal. } \\ \text { d. } & \text { Muito peque(ni)ninha. }\end{array}$

A primeira produção que aparece de muito + verbo nos dados da criança $L$ é somente aos 3 anos e 10 meses:

(290) Eu gosto [/] eu gosto muito de presente também.

(L 3;10;07)

A partir dessa idade, $L$ passa a produzir sentenças com o advérbio muito modificando vários tipos de verbos:

(291) a. E o dia demora muito.

b. Mas eu num lembro muito.

c. A gente só brincou muito assim xxx.

d. (Es)tá chovendo muito.

Logo, os dados da criança $L$ apoiam a hipótese de que as crianças produzem modificação de grau com adjetivo antes da modificação de grau com verbos. Desse modo, os dados de aquisição sustentam, em princípio, a assunção de partida deste trabalho de que a modificação de grau de adjetivos é uma operação menos complexa que a modificação de grau de verbos.

A terceira consequência é a importância da indeterminação para a tipologia dos predicados graduáveis. A seção 3.3 defendeu que para pita(t) ser apropriadamente analisando quando modifica predicados atélicos, é preciso levar em conta escalas indeterminadas. Logo, 
a indeterminação é tão importante para a tipologia dos predicados graduáveis quanto a abertura das escalas associadas a eles e a sua relação com um parâmetro de comparação (ser relativo ou absoluto). É exatamente nesse ponto que se encontra uma das contribuições teóricas deste trabalho. Além dos parâmetros conhecidos baseados na distinção escala fechada versus escala aberta e predicado relativo versus predicado absoluto, a propriedade determinado versus indeterminado também é importante.

$\mathrm{Na}$ realidade, uma vez que há uma correspondência um para um entre os dois parâmetros descritos em Kennedy e McNally (2005), não há razão para considerá-los como dois parâmetros diferentes. Nossa proposta é que eles podem ser tratados como um único parâmetro descrito de duas maneiras diferentes. A distinção relativo-absoluto é uma característica dos predicados graduáveis e a distinção aberto-fechado é uma propriedade de suas escalas. Por outro lado, a indeterminação é um parâmetro independente, uma vez que permite combinação cruzada com os outros. A tabela abaixo resume a proposta e apresenta exemplos.

Tabela 53: Proposta de Tipologia dos Predicados Graduáveis

\begin{tabular}{|c|c|c|c|}
\hline & (In)determinação & \multicolumn{2}{|c|}{ Exemplo } \\
\cline { 3 - 4 } da Escala & Adjetivo & Verbo \\
\hline Predicado Relativo & Determinado & alto & esfriar (leitura atélica) \\
\cline { 3 - 4 } Escala Aberta & Indeterminado & grande & correr \\
\hline $\begin{array}{c}\text { Predicado Absoluto } \\
\text { Escala Fechada }\end{array}$ & Determinado & aberto & chegar \\
\hline
\end{tabular}

A tabela acima mostra que os predicados relativos, ou de escala aberta podem ser divididos em dois tipos: os de escala determinada, e os de escala indeterminada. Os predicados de escala determinada são os predicados já discutidos pela Semântica Escalar. Um exemplo de adjetivo é alto. Trata-se de um predicado de escala aberta porque não há um limite lexicalmente determinado para o máximo de altura e de um predicado de escala determinada porque ele deve ser avaliado segundo uma escala de altura. $\mathrm{O}$ verbo esfriar em sua leitura atélica é um exemplo de predicado verbal desse tipo. Hay, Kennedy e Levin (1999) trataram desses verbos como derreter e esfriar, chamados de degree achievements, que possuem ora uma leitura télica, ora uma leitura atélica, como se pode ver no exemplo abaixo. 
Em (292a) o verbo to cool tem interpretação atélica, ou seja, a sentença quer dizer que a sopa esfriou, mas que não ficou necessariamente fria. Já em (292b), a sentença informa que a sopa esfriou até atingir o grau de frio.

(292) a. The soup cooled for an hour.

'A sopa esfriou por uma hora'

b. The soup cooled in an hour.

'A sopa esfriou em uma hora'

Nesse sentido, o verbo to cool 'esfriar' pode ser um predicado de escala aberta, quando tem leitura atélica, ou um predicado de escala fechada, quando tem uma leitura télica. De todo modo, trata-se de um predicado de escala determinada, a escala de resfriamento. Essa determinação da escala pode estar relacionada com o fato de haver uma relação intrínseca entre a entrada lexical do verbo e do adjetivo que caracteriza o estado resultante do processo. Esfriar é derivada de frio. Já em inglês, o verbo e o adjetivo são representados pela mesma palavra: cool.

Predicados de escala fechada sempre têm escala determinada. Na tabela acima, os adjetivos são representados por aberto que é avaliado segundo um grau de abertura. Um exemplo de verbo é o chegar que, como vimos, pode estar associado a uma escala fechada que representa a progressão do evento. O grau máximo dessa escala é o telos do evento. A novidade nesta tabela, inserida pela contribuição deste trabalho, está na existência de predicados relativos, que têm escala aberta, e que possuem uma escala indeterminada. O caso típico discutido nesse trabalho é correr. Como vimos esse verbo pode ter uma versão graduável no curso da derivação semântica, por meio de uma função de grau Deg, mas não possui, no entanto uma escala corridade. A escala nesse tipo de predicado é dada contextualmente. Como representante dos adjetivos, selecionamos grande por parecer possuir, pelo menos parcialmente, essa característica. Grande pode ser avaliado segundo uma escala de altura, em uma sentença como Este prédio é grande, ou em uma escala de espessura, como em Este livro é grande, ou de ainda volume quando se diz Eu comprei um refrigerante grande.

A quarta e última consequência da proposta defendida neste trabalho também diz respeito a uma potencial contribuição teórica. A proposta de entrada lexical para pita(t) 
baseada na semântica de pos é uma evidência de que há uma semelhança entre o grau normal de escalas abertas e o grau máximo de escalas fechadas quando são codificadas nos predicados graduáveis nas línguas naturais. Embora pita(t) não seja exatamente a função pos pronunciada, ele é um modificador que seleciona esses dois graus, representados por $\mathbf{d}_{\mathbf{s}}$ na proposta de Kennedy (2007). Ele é, portanto, uma evidência de que esses graus devem ser representados como um só. Seu traço é o que chamamos de ponto de corte da escala. Ou seja, é grau que determina o corte na escala entre os graus que mapeiam indivíduos que não possuem a característica designida pelo predicado e os que possuem.

$$
\left[[\text { pita }]=\lambda \mathrm{G}_{<\mathrm{d},<\mathrm{e}, \triangleright>} \lambda \mathrm{x}_{\mathrm{e}} . \exists \mathrm{d}\left[\mathrm{G}(\mathrm{d})(\mathrm{x}) \& \mathrm{~d} \geq \mathrm{d}_{\mathrm{s}}\right]\right.
$$

Portanto, esse trabalho tem pelo menos duas contribuições teóricas, a importância da indeterminação para a tipologia das escalas e uma evidência de que os graus normal e máximo das escalas são semelhantes em mais construções além da forma positiva do adjetivo. 


\section{CONSIDERAÇÕES FINAIS}

Nesta seção, apresento as conclusões e considerações finais acerca das propostas contidas nesta tese. O objetivo central desta tese foi o de apresentar uma análise para a modificação de grau em Karitiana investigando o modificador pita(t) na língua. A tese foi dividida em quatro capítulos. Embora a proposta tenha aparecido de forma explícita somente no quarto capítulo, cada um dos capítulos contribuiu para a apresentação da proposta defendida.

O primeiro capítulo descreveu, de maneira geral, o pano de fundo desta pesquisa. Seu objetivo principal foi o de familiarizar o leitor com as características da língua Karitiana e com o pano de fundo teórico adotado na tese. As características da língua Karitiana apresentadas nos auxiliaram na apresentação dos dados durante o desenvolvimento da argumentação. As propriedades singulares da língua quanto à marcação de tempo e de aspecto e aos sintagmas nominais, por exemplo, foram retomadas ao longo do texto para contribuir com a análise dos sintagmas verbais quanto à distinção télico-atélico e, por consequência, da modificação de grau nesse domínio.

O capítulo procurou, ainda, esclarecer quais são as perguntas envolvidas na investigação de uma língua segundo o paradigma teórico da Semântica Formal, deixando claro que a questão central desta tese foi a de explicitar qual a contribuição semântica do advérbio pita(t) para o significado das sentenças em que ocorrem. Esta seção serviu para a apresentação das ferramentas formais básicas utilizadas neste trabalho. A apresentação da semântica composicional e da semântica de eventos serviram de pano de fundo para a forma como a análise se delineou durante a argumentação da tese. Duas propostas defendidas na tese ilustram o seu uso: a denotação de pita(t) defendida é uma função que se aplica aos predicados modificados, o que demonstra o estatuto composicional da proposta; e (ii) o sufixo $\{-t\}$ é um morfema que insere um tipo s de eventos, o que apoia a adoção de uma semântica de eventos.

O capítulo 2 apresentou uma descrição e uma justificativa para a metodologia adotada nesta pesquisa. Trata-se de uma reflexão sobre como os dados devem ser coletados para uma pesquisa em Semântica Formal. Isto é importante porque esclarece pontos não consensuais a respeito do trabalho de campo em linguística. Defendemos que o pano de fundo teórico orienta o métododo de coleta de dados linguísticos. Em Semântica Formal, os dados 
utilizados para a análise precisam conter sentenças acompanhadas de descrições dos contextos que descrevem adequadamente suas condições de verdade, que representam seu significado. Para coletar dados desse tipo, é preciso utilizar uma metodologia apropriada. Defendemos que a metodologia mais adequada para os trabalhos de campo de uma pesquisa em Semântica Formal é a elicitação controlada. Trata-se da realização de entrevista com consultores nativos na língua investigada por meio de perguntas acerca da tradução de sentenças e de sua aceitabilidade em determinados contextos.

Além disso, o capítulo defendeu uma proposta de mudança na metodologia de elicitação controlada adotada usualmente pela Semântica Formal. Defendemos que na fase de elicitação de contextos, leve-se em conta os que estamos denominando de contextos limítrofes. Os contextos limítrofes são os contextos que auxiliam a explicitar quais os aspectos do significado das sentenças devem ser considerados como parte do significado convencional (sua semântica) e quais aspectos podem ser descritos como inferências pragmáticas. Essa proposta contribui para o panorama geral da pesquisa em Semântica Formal, com línguas pouco descritas ou não, porque apresenta uma ferramenta formal que auxilia na argumentação de uma análise acerca do significado.

O capítulo 3 apresentou uma investigação dos sintagmas verbais em Karitiana. A investigação foi importante porque esclareceu propriedades desses sintagmas que foram úteis para a análise da modificação de grau nesse domínio. A primeira parte do capítulo discutiu a pertinência de se fazer uma distinção dos predicados verbais na língua entre as classes acionais adotadas na literatura, mais especificamente, quanto à distinção télico-atélico. Um conjunto de testes foi aplicado e discutido em Karitiana. O capítulo mostrou que, embora os testes clássicos da literatura para diagnosticar as classes verbais não são possíveis de ser aplicados na primeira tentativa, isso não quer dizer que a língua não possui essas distinções. Os testes devem ser repensados e adaptados levando-se em conta as características particulares da língua. Uma reflexão acerca das características dos nomes nus, que são neutros para número, e da marcação de tempo não futuro, que pode ser usada para descrever eventos perfectivos e imperfectios, ajudou a re-elaborar os testes visando a encontrar um diagnóstico seguro para a identificação dos diferentes predicados. A diferença entre predicados télicos e atélicos foi comprovada na língua, o que contribuiu para a análise da modificação de grau nesse domínio aparesentada no capítulo 4. A distinção télico-atélico foi utilizada para associar os predicados verbais a predicados graduáveus de escalas fechadas e abertas. A diferença é 
crucial para o uso de pita(t) nesse domínio, que tem uma interpretação de 'muito' quando modifica predicados atélicos (considerados de escala aberta) e de 'mesmo' quando modifica predicados télicos (considerados de escala fechada).

A segunda parte do capítulo discutiu as propriedades semânticas da cumulatividade e da contabilidade associadas aos predicados verbais na língua. Argumentamos que não há contradição na afirmação de que o domínio verbal em Karitiana tem denotação ao mesmo tempo cumulativa e contável e exploramos as consequências dessa afirmação. A aparente contradição da afirmação está no fato de haver uma associação recorrente da propriedade da cumulatividade a predicados massivos. As consequências dessa afirmação para a proposta geral defendida na tese dizem respeito, sobretudo, à possibilidade de contabilidade no domínio verbal. Essa possibilidade é explorada na proposta oferecida para pita(t) de que a leitura iterativa das sentenças com predicados atélicos modificadas pelo advérbio é uma das escalas possíveis, que está disponível exatamente porque esse predicado também é considerado um predicado contável.

Finalmente, no quarto capítulo, a modificação de grau com o advérbio pita(t) foi analisada com uma teoria que envolve escalas e graus. A sua maior contribuição foi a de fornecer uma entrada lexical para o modificador que deriva adequadamente o seu significado nos diferentes domínios. A tese defendida foi a de que pita(t) é um modificador de grau nos domínios nominal, adjetival, e verbal, que sempre seleciona um predicado com um argumento de grau. Pita é o modificador de substantivos e adjetivos e pitat é o modificador sobre predicados verbais. O sufixo $\{-t\}$ é sintaticamente um adverbializador e semanticamente insere o tipo s de eventos na denotação de pita.

Pita(t) tem interpretações diferentes com diferentes tipos de predicado. Com substantivos, ele é interpretado como algo como 'verdadeiro'. Com adjetivos, ele tem uma interpretação semelhante à do muito do português, mas também como à de completamente. No domínio verbal, ele se comporta ora como o muito do português e ora como uma confirmação, algo como mesmo. A tese mostrou que essa variabilidade não é aleatória, mas está relacionada com o tipo do predicado modificado.

Com a proposta de divisão dos predicados entre predicados de escala aberta e fechada, foi possível entender que a distribuição de pita(t) com predicados adjetivais e vebais é bastante semelhante. Quando pita(t) modifica um predicado que está assciado a uma escala aberta, ele expressa algo do tipo 'acima de um grau normal da escala'. Quando modifica 
predicados associados a escalas fechadas, ele exprime alguma coisa do tipo 'no grau máximo da escala'. Por conta disso, quando pita(t) modifica adjetivos de escala aberta e predicados verbais atélicos (considerados de escala aberta), ele é interpretado o muito do português, que significa algo como 'acima de um grau normal da escala'. Já quando modifica adjetivos de escala fechada e predicados verbais télicos (considerados de escala fechada), pita(t) é interpretado como 'no grau máximo da escala', daí sua tradução para completamente com os adjetivos de escala fechada. Com os predicados verbais télicos, o grau máximo da escala é o telos do evento. Portanto, atingir o grau máximo da escala para esses predicados significa dizer que o telos foi atingido, ou seja, que o evento foi completado. Daí a sua tradução para mesmo em português. Quanto aos substantivos, vimos que é possível apresentar uma proposta na mesma linha se considerarmos que eles estão asssociados a uma escala fechada de precisão. Assim, quando pita modifica substantivos, ele é interpretado como 'no grau máximo na escala de precisão'.

A proposta para o significado de pita(t) foi feita com base na proposta de Kennedy (2007) para o morfema nulo pos. Mais especificamente, assumimos a proposta de um grau $\mathbf{d}_{\mathbf{s}}$ que representa o grau de corte das escalas. O grau de corte é o grau normal se o predicado for de escala aberta, e o grau máximo se o predicado for de escala fechada. Esse é exatamente o grau selecionado por pita(t). Adotamos a tese de que a contribuição semântica de pita(t) é a mesma em todos os domínios: a de que há um grau maior ou igual ao grau $\mathbf{d}_{\mathbf{s}}$. Para implementar formalmente a proposta, postulamos uma função Deg que fornece um argumento de grau e uma variável de escalas para predicados verbais e uma função Deg $_{\text {subst }}$ que fornece um argumento de grau e uma escala de precisão para substantivos. Dessa forma, foi possível apresentar uma entrada lexical unificada para pita(t) que captura adequadamente as condições de verdade das sentenças em que ocorre.

Esta tese mostrou que uma teoria formalista pode auxiliar na análise de uma língua pouco descrita pela literatura. Conforme o texto mostrou, muitas vezes, é difícil escolher qual o modo mais adequado de traduzir uma palavra, ou de glosar um morfema. O modificador pita(t) pode ser traduzido por verdadeiro, muito, completamente, mesmo, ou em alguns casos nem precisa ser traduzido. Isso é resultado do fato de que os significados de sentenças, operadores e palavras não são equivalentes quando se compara línguas diferentes. Com o uso do formalismo, é possível explicitar o significado de um operador. As composições semânticas e as formas lógicas tornam clara a contribuição semântica do modificador que 
fica, muitas vezes, obscurecida nas traduções. Nesse sentido, defendemos como proposta geral que, embora a análise de uma língua pouco descrita por uma abordagem formalista pareça, muitas vezes, uma tarefa difícil, ela é bastante adequada porque consegue expressar e capturar diferenças melhor que qualquer paráfrase ou explicação. 


\section{REFERÊNCIAS}

ABBI, A. A Manual of Linguistic Field Work and Indian Language Structures. Munich: Lincom Europa, 2001.

AIKHENVALD, A. Linguistic fieldwork: setting the scene. STUF - Sprachtypologie und Universalienforschung, 60 (1), p. 3-11, 2007.

ARIEL, M. Pragmatics and Grammar. Cambridge: Cambridge University Press, 2008.

BACH, E. The Algebra of Events. Linguistics and Philosophy 9, p. 5-16, 1986.

. Informal Lectures in Informal Semantics. Nova Iorque: State University of New York Press, 1989.

BACH, E.; JELIEK, E.; KRATZER A.; PARTEE, B. (eds.) Introduction. In: Quantification in Natural Languages. Boston: Kluwer Academic Publishers, 1995.

BASSO, R. Telicidade e detelicização: semântica e pragmática do domínio tempo-aspectual. 2007. Dissertação (Mestrado em Linguística) - Instituto de Estudos da Linguagem, Universidade Estadua de Campinas, Campinas. Disponível em: $<$ http://www.bibliotecadigital.unicamp.br/document/?code=vtls000406707 >. Acesso em: 06 mai de 2013.

BURZIO, L. Italian Syntax. Dordrecht: Reidel, 1986.

BOCHNAK, M. R. Two sources of scalarity within the verb phrase. Trabalho apresentado no Workshop on Subatomic Semantics of Event Predicates. 2010.

BOWERN, C. Linguistic Fieldwork: A Practical Guide. Nova Iorque: Palgrave MacMillan, 2008.

CARLSON, G. Reference to kinds in English. Nova Iorque e Londres: Garland Publishing, 1980.

CARVAlHO, A. M. O Auxiliar Aspectual Tyka do Karitiana. Revista Letras, n. 78, maio/ago, 2009. 
. O auxiliar aspectual Tyka do Karitiana. 2010. Dissertação (Mestrado em Semiótica e Linguística Geral) - Faculdade de Filosofia, Letras e Ciências Humanas, Universidade de São Paulo, São Paulo. Disponível em: $<$ http://www.teses.usp.br/teses/disponiveis/8/8139/tde-25082010-144136/en.php>. Acesso em: 06 mar de 2013.

CAUDAL, P.; NICOLAS, D. Types of degrees and types of event structures. In: MAIENBORN, C.; WÖLLSTEIN, A. Event Arguments: Foundations and Apllications. Tübingen: Niemeyer, 2005.

CHELLIAH, S. L.; DE REUSE, W. J. Handbook of Descriptive Linguistic Fieldwork. Nova Iorque: Springer, 2011.

CHIERCHIA, G. Plurality of mass nouns and the notion of "semantic parameter". In: ROTHSTEIN, S. (ed.). Events and grammar. Dordrecht: Kluwer, 1998. p. 53-103.

. Semântica. Tradução de Luis Arthur Pagani, Lígia Negri, Rodolfo Ilari. Londrina: Eduel; Campinas: Editora da Unicamp, 2003.

COMRIE, B Aspect: An introduction to the study of verbal aspect and related problems. Cambridge: Cambridge University Press, 1985.

COUTINHO-SILVA, T. Aspectos dos Sintagmas Nominais em Karitiana: a Quantificação Universal. 2008. Dissertação (Mestrado em Semiótica e Linguística Geral) Faculdade de Filosofia, Letras e Ciências Humanas, Universidade de São Paulo, São Paulo. Disponível em: <http://www.teses.usp.br/teses/disponiveis/8/8139/tde24092009-101219/>. Acesso em: 03 jul de 2012.

CROWLEY, T. Field Linguistics: A Beginner's Guide. Oxford: Oxford University Press, 2007.

DAVEY, B. A. PRIESTLEY, H. A. Introduction to Lattices and Order. Cambridge: Cambridge University Press, 1990.

DAVIDSON, D. The Logical Form for Action Sentences. In: RESCHER, N. (ed.) The Logic of Decision and Action. Pittsburgh: University of Pittsburgh Press, 1967. p. 81-95.

DOETJES, J. Adverbs and quantification: degree versus frequency. Lingua 117, p. 685-720, 2007. 
DONAZZAN, M. On counting and measuring events. In: CHEMLA, E.; HOMER, V.; WINTERSTEIN, G. (eds.) Proceedings of Sinn und Bedeutung 17. 2013 Disponível em: <http://semanticsarchive.net/sub2012/SuB2013.pdf>. Acesso em: 19 dez. 2013.

DOWTY, D. R. Word Meaning and Montague Grammar. Reidel: Dordrecht, 1979.

EVERETT, C. Patterns in Karitiana: Articulation, perception, and grammar. 2006. Tese (Doutorado em Linguística) - Rice University, EUA.

EVERETT, D. A note on ergativity $S^{\prime}$ e $S$ " in Karitiana. In: DERBYSHIRE, D. (ed.) Workpapers of the Summer Institute of Linguistics. Grand Forks: University of North Dakota, v. 29, 1985.

- Case Spreading and Phrase Structure in Karitiana. In; HARGUS, S.; McMENNBAMIN, G. R.; SAMIIAN, V. (eds.) Proceedings of WECOL 93 (Western Conference on Linguistics). California State University, Fresno, 1993.

. Monolingual fieldwork research. In: NEWMAN, P.; RATLIFF, M. (eds.) Linguistic Fieldwork. Cambridge: Cambridge University Press, 2001. p.166-188.

FERREIRA, M. Event Quantification and Plurality. 2005. Tese (Doutorado em Linguística) Massachusetts Institute of Technology, EUA.

FILIP, H.; ROTHSTEIN, S. Telicity as a semantic parameter. In: LAVINE, J.; FRANKS S.; FILIP, H.; TASSEVA-KURKTCHIEVA, M. (eds.) Formal Approaches to Slavic Linguistics 14: The Princeton University Meeting. Ann Arbor: Michigan Slavic Publications, 2005.

FREGE, G. Über Begriff und Gegenstand. Vierteljahresschrift für wissenschaftliche Philosophie 16: 192-205. 1892. Traduzido para o português como "Sobre o Conceito e o Objeto", in Lógica e Filosofia da Linguagem; trad. Paulo Alcoforado. - São Paulo: Cultrix, 1978.

FRUTOS, L. Escalas no Guarani Paraguaio: uma análise do modificador de grau -pa. 2011. Dissertação (Mestrado em Semiótica e Linguística Geral) - Faculdade de Filosofia, Letras e Ciências Humanas, Universidade de São Paulo, São Paulo.

GUIMARÃES, M. R. Dos intensificadores como qualificadores: os âmbitos de expressão da quantificação no português do Brasil. 2007. Tese (Doutorado em Linguística) UFPR, Curitiba. 
GRICE, P. Studies in the Way of Words. Harvard: Harvard University Press, 1989.

. Logic and Conversation. In: COLE, P.; MORGAN, J. L. (eds.) Syntax and Semantics 3: Speech Acts. Nova Iorque: Academic Press, 1975. p. 41- 58.

HAY, J.; KENNEDY, C; LEVIN, B. Scalar Structure Underlies Telicity in 'Degree Achievements'. Proceedings of SALT 9. Nova Iorque: Cornell Linguistics Circle Publications, Cornell University, 1999. p. 127-144.

HEIM, I.; KRATZER, A. Semantics in generative grammar. Oxford: Blackwell Publishers, 1998.

HORN, L. On the semantic properties of the logical operators in English. Bloomington: Indiana University Linguistics Club, 1972.

KENNEDY, C. Projecting the adjective: The syntax and semantics of gradability and comparison. NewYork: Garland. 1999.

- Vagueness and Grammar: The Semantics of Relative and Absolute Gradable Adjectives. Linguistics and Philosophy 30.1, 2007.

KENNEDY, C.; LEVIN, B. Measure of Change: The Adjectival Core of Verbs of Variable Telicity. In: MCNALLY, L.; KENNEDY, C. (ed.) Adjectives and Adverbs in Semantics and Discourse. Oxford: Oxford University Press, 2008. p. 156-182.

KENNEDY, C.; MCNALLY, L. Scale Structure, Degree Modification, and the Semantics of Gradable Predicates. Language 81, n.2, p. 345-381, 2005.

KLEIN, E. A semantics for positive and comparative adjectives. Linguistics and Philosophy 4, p. $1-45,1980$.

KLEIN, W. Time In Language. Londres, Nova Iorque: Routledge, 1994.

KRATZER, A. Stage-Level and Individual-Level-Predicates. In CARLSON, G. N.; PELLETIER, F. J. (eds.) The Generic Book. Chicago: University of Chicago Press, 1995. p. 125-175.

. Severing the External Argument from its Verb. In: ROORYCK, J.; ZARING L. (eds.) Phrase Structure and the Lexicon. Dordrecht: Kluwer Academic Publishers, 1996. p. 109-137. 
. The Event Argument and the Semantics of Verbs. 2003. Manuscrito disponível em: $<\mathrm{http}: / /$ semanticsarchive.net>. Acesso em: 10 mai. 2008.

. On the Plurality of Verbs. In: DÖLLING, J.; HEYDE-ZYBATOW, T. (eds.) Event Structures in Linguistic Form and Interpretation. Berlin: Mouton de Gruyter, 2007.

KRIFKA, M. Thematic Relations as Links between Nominal Reference and Temporal Constitution. In: SAG, I.; SAZBOLSCI. (eds.) Lexical Matter. Chicago: CSLI, 1992. p. 29-53.

. Verkuyk, Henk J. A Theory of Aspectuality. The Interaction between Temporal and Atemporal Structure. Studies in Language 20:2, p. 443-454, 1996.

. The origins of telicity. In: ROTHSTEIN, S. (ed.) Events and grammar. Dordrecht: Kluwer, 1998. p. 197-235.

LANDIN, D. Dicionário e Léxico Karitiana/Português. Brasília: SIL, 1983.

. An Outline of the Syntactic Structure of Karitiana Sentences. Estudos Sobre Linguas Tupi do Brasil. Série Linguística 11. Brasília: SIL, 1984.

. As Orações Karitiana. Série Linguística 9 (2). Brasília: SIL, 1988.

LANDIN, R. Word Order Variation in Karitiana. Arquivo Linguístico no. 149. Brasília: SIL, 1982.

. Conjunções Karitiana de Nível Superior. Série Linguística 9(1). Brasília: SIL, 1987.

. Kinship and Naming Among the Karitiana of Northwestern Brazil. 1989. Dissertação (Mestrado em Linguística). University of Texas, EUA.

LANDIN, D.; LANDIN. R. A Preliminary Description of the Karitiana Phonological Structure. Arquivo Linguístico No. 163. Brasilia: SIL, 1973.

LASERSOHN, P. Plurality, Conjunction, and Events. Boston: Kluwer Academic Publishers, 1995.

. Pragmatic halos. Language 75, p. 522-551, 1999.

LINK, G. The Logical Analysis of Plurals and Mass Terms: A Lattice-theoretical Approach. In: BÄUERLE, R.; SCHWARZE, C. STECHOW, A. (eds.) Meaning, Use, and Interpretation of Language. Berlin, Nova Iorque: Springer, 1983. p. 302-323. 
MALOSSO, M. Núcleos Funcionais em Karitiana. Relatório de Pesquisa não publicado. 2003

MARANTZ, A. On the Nature of Grammatical Relations. Cambridge: MIT Press, 1984.

MASIÀ, M. What veracity does to imprecision: the case of Spanish verdadero. Proccedings of the ESSLLI 2013 Student Session, Düsseldorf, Alemanha, 2013.

MATTHEWSON, L. On the Methodology of Semantic Fieldwork. International Journal of American Linguistics 70, p. 369-415, 2004.

MCNALLY, L. Modification. In: ALONI, M.; DEKKER, P. (eds.) Cambridge Handbook of Semantics. Cambridge: Cambridge University Press, no prelo.

MONTAGUE, R. English as a formal language. In: VISENTINI, B. (ed.) Linguaggi nella societa e nella tecnica. Milan: Edizioni di Communita, 1970. p. 189-224.

. The Proper Treatment of Quantification in Ordinary English. In: HINTIKKA, J.; MORAVCSIK, J.; SUPPES, P. (eds.) Approachs to Natural Language: Proceedings of the 1970 Stanford Workshop on Grammar and Semantics (Synthese Library). Dordrecht - Holanda, Boston - EUA, D. Reidel Publishing Company, 1973. p. 221242.

MORZYCKI, M. Metalinguistic comparison in an alternative semantics for imprecision. Natural Language Semantics 19(1), 39-86, 2011.

MÜLLER, A. The Expression of Genericity in Brazilian Portuguese. In: KUSUMOTO, K.; VILLALTA, E. (eds.) UMOP23: Issues in Semantics. Amherst: GLSA Graduate Linguistics Student Association, 2000.

. Variação semântica: individuação e número na língua Karitiana. Estudos Linguísticos 38, p. 295-308, 2009.

. On the Encoding of the Definite/Indefinite Distinction in Karitiana. In: REICH, I. et al. (Ed.) Proceedings of Sinn \& Bedeutung 15. Universaar - Saarland University Press: Saarbrücken, Alemanha, 2011.

. Distributividade: O Caso dos Numerais Reduplicados em Karitiana. Cadernos de Estudos Linguísticos, 54.2, Campinas, jul./dez., 2012. 
MÜLLER, A.; NEGRÃO, E. On Distributivity in Karitiana. In: CABREDO-HOFHERR, P.; LACA, B. (eds.) Verbal Plurality and Distributivity. Berlin: Mouton de Gruyter, 2012 .

MÜLLER, A.; SANCHEZ-MENDES, L. Pluractionality in Karitiana. In: GRØNN, A. (ed.) Proceedings of SuB12, Oslo: Department of Literature, Area Studies and European Languages, University of Oslo, p. 442-454, 2008.

. O Significado da Pluracionalidade em Karitiana. Cadernos de Estudos Linguísticos, v. 52,2, p. 215-231, 2010.

MÜlleR, A.; STORTO, L.; COUTINHO-SILVA, T. Número e a Distinção ContávelMassivo em Karitiana. Revista da Abralin, v. 5, p. 185-213, 2006.

NAKANISHI, K. Formal Properties of Measurement Constructions. Berlin: Mouton de Gruyter, 2007.

NEWMAN, P.; RATLIFF, M. Linguistic Fieldwork. Cambridge: Cambridge University Press, 2001.

PARIS, M. C. Verbal reduplication and verbal classifiers in Chinese. In: DJAMOURI, R.; CHAPPELL, H.; WIEBUSCH, T. (eds.) Festschrift in honor of Alain Peyraube. Taipei: Academia Sinica, 2011.

PARSONS, T. Events in the Semantics of English: A Study in Subatomic Semantics. Cambridge: MIT Press, 1990.

PARTEE, B.; BACH, E.; KRATZER, A. Quantification: A Cross-Linguistic Perspective. Amherst: NSF proposal, University of Massachusetts, 1987.

PIÑÓN, C. Achievements in an Event Semantics. LAWSON, A. (ed.) SALT Vll, Ithaca, NY: Cornell University, 1997.

. Happening gradually. In: CONATHAN, L.; GOOD, J.; KAVITSKAYA, D.; WULF, A.; YU, A. (ed.) Proceedings of BLS 26. Berkeley Linguistics Society, 2000.

PIRES DE OLIVEIRA, R. Uma História de Delimitações Teóricas: Trinta Anos de Semântica no Brasil. D.E.L.T.A., vol. 15, 1999.

QUINE, W. V. O. Word and Object. Cambridge: MIT Press, 1960. 
RAPPAPORT HOVAV, M.; LEVIN, B. Reflections on Manner/Result Complementarity. In: DORON, E.; RAPPAPORT HOVAV, M.; SICHEL, I. (ed.) Syntax, Lexical Semantics, and Event Structure. Oxford: Oxford University Press, 2010. p. 21-38.

REINCHENBACH, H. Elements of Symbolic Logic. Londres: MacMillan, 1947.

RICE, K. Ethical Issues in Linguistic Fieldwork. In: THIEBERGER, N. (ed.) The Oxford Handbook of Linguistics Fieldwork. Oxford: Oxford University Press, 2012.

ROCHA, I. A estrutura Argumental da Lngua Karitiana: Desafios Descritivos e Teóricos. 2011a. Dissertação (Mestrado em Semiótica e Linguística Geral) - Faculdade de Filosofia, Letras e Ciências Humanas, Universidade de São Paulo, São Paulo. Disponível em: <http:/www.teses.usp.br/teses/disponiveis/8/8139/tde-12092012120027/pt-br.php>. Acesso em: 06 mar de 2013.

. Estudo da estrutura argumental dos verbos da língua Karitiana. EPOG - Encontro de Pós-Graduandos da FFLCH/USP, 2011, São Paulo. VI EPOG. São Paulo: Serviço de Divulgação e Informação - FFLCH, 2011b.

. Estrutura argumental em Karitiana: Verbos Intransitivos com Objeto Oblíquo. In: NAVES, R. R.; SALLES, H. M. M. (orgs.) Estudos Formais da Gramática das Linguas Naturais: artigos selecionados do Encontro Nacional do Grupo de Trabalho Teoria da Gramática/2009. Goiânia: Cânone, 2011c. p. 478-487.

. Verbos intransitivos alternantes em Karitiana à luz da teoria de estrutura argumental de Hale e Keyser (2002). In: BAIA, M. F. A.; OUSHIRO, L. NASCIMENTO, I.B. (eds.) Anais dos XII e XIII Encontros dos Alunos de Pós-Graduação em Linguística da USP. São Paulo: Paulistana, 2012. p. 163-173.

. Valência Verbal e núcleos aspectuais em orações subordinadas em Karitiana: o caso das relativas e adverbiais. Trabalho apresentado no II Workshop de Línguas Indígenas da USP. 2013.

ROTHSTEIN, S. Fine-grained structure in the eventuality domain: the semantics of predicate adjective phrases and 'be'. Natural Language Semantics 7, p. 347-420, 1999. . Structuring events: a study in the semantics of lexical aspect. Oxford: Blackwell, 2004. 
. Telicity, atomicity and the Vendler classification of verbs. In: ROTHSTEIN, S. (ed.) Theoretical and Crosslinguistic Approaches to the Semantics of Aspect. Amsterdam: John Benjamins, 2008. p. 43-78.

. Counting and the Mass Count Distinction. Journal of Semantics 27(3), p. 343-397, 2010.

SANCHEZ-MENDES, L. A Aquisição de um Modificador de Grau em Português Brasileiro. Trabalho não publicado.

. A Expressão da Quantificação em Karitiana. Caderno de Pesquisa na Graduação em Letras - Revista da Associação Nacional de Pesquisa na Graduação em Letras. Ano III, número 3, p. 103-110, 2006.

A Quantificação Adverbial em Karitiana: os Advérbios. In: MINUSSI, R. D.; ROCHA, S. Anais do X Encontro dos Alunos de Pós-Graduação em Linguística, São Paulo-SP, 2008.

. A Quantificação Adverbial em Karitiana. 2009. Dissertação (Mestrado em Semiótica e Linguística Geral) - Faculdade de Filosofia, Letras e Ciências Humanas, Universidade de São Paulo, São Paulo. Disponível em: $<\mathrm{http}: / /$ www.teses.usp.br/teses/disponiveis/8/8139/tde-23112009-104847/>. Acesso em: 22 jun de 2012.

. Quantificação adverbial: operações de frequência e de grau. Estudos Linguísticos, São Paulo, v. 40, p. 395-404, 2011.

. Cumulativity and Count Properties in Karitiana Verbs. In: BOGAL-ALLBRITTEN, E. (ed.) Sula 6: Proceedings of the Sixth Conference on the Semantics of Underrepresented Languages in the Americas and SULA-Bar. Amherst: GLSA Graduate Linguistics Student Association, 2012a.

- Scalar Properties of Degree Modification in Karitiana: Evidence for Indeterminate Scales. In: RENDSVIG, R. K.; KATENKO, S. (eds.) Proccedings of the ESSLLI 2012 Student Session, Opole, Polônia, 2012b. 
SANCHEZ-MENDES, L.; MÜLLER, A. The Expression of Quantification in Karitiana: Pluractionality and Quantifiers. In: DEAL, A. R. (ed.) UMOP 35: Proceedings of the 4th Conference of semantics of under-represented languages in the Americas. Amherst: BookSurge Publishing, 2007.

SAPIR, E. Grading: a Study in Semantics. Philosophy of Science 11, p. 93-116, 1944.

STORTO, L. Basic Word Order in Karitiana. In: LANGDON, M. (ed.) Survey Reports 8: Proceedings of the Meeting of the Society for the Study of the Indigenous Languages of the Americas July 2-4, 1993 and the Hokan-Penutian Workshop July 3, 1993. Linguistic Institute at Ohio State University in Columbus, 1994.

. A Report on Language Endangerment in Brazil. In: BOBALJIK, R.; PENSALFINI, R.; STORTO, L. (eds.) MIT Working Papers in Linguistics 28: Papers on Language Endangerment and the Maintenance of Linguistic Diversity. Cambridge, MA, USA, 1996.

- Karitiana: A verb Second Language from Amazonia. In: Sixth Conference of Students of Linguistics of Europe (CONSOLE). 1998.

. Aspects of Karitiana Grammar. 1999. Tese (Doutorado em Linguística) - MIT, EUA.

. Duas Classes de Verbos Intransitivos em Karitiana (Família Arikém, Tronco Tupi). In: QUEIXALOS, F. (org.) Des noms et des verbes en tupi-guarani: état de la question. Muenchen: Lincom-Europa, 2001.

- Algumas Categorias Funcionais em Karitiana. In: CABRAL, A. S. A. C.; RODRIGUES, A. D. (org.) Linguas Indígenas Brasileiras: Fonologia, Gramática e História: Atas do I Encontro Internacional do Grupo de Trabalho sobre Línguas Indigenas da ANPOLL. Belém, Pará : Editora Universitária UFPA, 2002.

- Interactions between verb movement and agreement in Karitiana. Revista Letras, Curitiba, n. 60, p. 411-433, 2003.

. Caso e Concordância nas Línguas Tupi. Estudos Linguísticos, Campinas. 2005.

. Marcação Absolutiva em Algumas Construções Sintáticas em Karitiana. Ameríndia (Revue d'Ethnolinguistique Amérindienne) 32: La Structure des Langues Amazoniennes, p. 183-203, 2008. 
. Copular Constructions in Karitiana: a case against case movement. In: LIMA. S. (ed.) University of Massachusetts Occasional Papers 41: Proceedings of SULA 5: Semantics of Under-Represented Languages in the Americas. Amherst: GLSA/The University of Massachusetts, 2010.

. Ausência de determinantes e complementizadores em Karitiana. In: NAVES, R. R.; LIMA-SALLES, H. R. N. (org.) Estudos Formais da Gramática das Línguas Naturais: artigos selecionados do Encontro Nacional do Grupo de Trabalho Teoria da Gramática/2009. Goiânia: Cânone Editorial, 2011.

. Duplicação em Karitiana. In: PIRES DE OLIVEIRA, R.; MEZARI, M. P. Nominais Nus: um olhar através das Linguas. Campinas: Mercado das Letras, 2012a.

. Subordination in Karitiana. Amerindia (Revue d'Ethnolinguistique Amérindienne) 35, p. 219-237, 2012b.

. Temporal and Aspectual Interpretations in Non-Finite Clauses. In: MOLSING, K. V.; IBAÑOS, A. M. T. (eds.) Time and TAME in Language. Newcastle upon Tyne: Cambridge Scholar Publishing, 2013.

. Reduplication in Karitiana. In: GÓMEZ, G. G.; VOORT, H. V. D. (eds.) Reduplicartion in Indigenous Languages of South America. Amsterdam: Brill, 2014. p. 398-423.

STORTO, L.; DEMOLIN, D. Pitch Accent in Karitiana. In: KAJI, S. (org.) Cross Linguistic Studies of Tonal Phenomena. Tokyo: Tokyo University of Foreign Studies, 2005. p. 329-356.

STORTO, L.; THOMAS, G. The Clausal Nature of Universally Quantified Phrases in Karitiana. In: BOGAL-ALLBRITTEN, E. (ed.) Sula 6: Proceedings of the Sixth Conference on the Semantics of Under-represented Languages in the Americas and SULA-Bar. Amherst: GLSA Graduate Linguistics Student Association, 2012.

STORTO, L.; VANDER VELDEN, F. F. Karitiana. In: Povos Indígenas do Brasil, 2005. Disponível em: <http://www.socioambiental.org/pib/epi/karitiana/karitiana.shtm>. Acesso em: 1 fev. 2009. 
THOMAS. G. Comparison across domains in Mbyá. Proceedings of WSCLA 14. Vancouver: Department of Linguistics, University of British Columbia, 2009.

VENDLER, Z. Verbs and times. The Philosophical Review 66.2, p. 143-160, 1957.

VERKUYL, H. J. A Theory of Aspectuality. The Interaction between Temporal and Atemporal Structure. Cambridge: Cambridge University Press, 1996.

YU, A. Pluractionality in Chechen. Natural Language Semantics 11, p. 289-321, 2003. 